\title{
Mechanism of Enyne Metathesis Catalyzed by Grubbs Ruthenium-Carbene Complexes: A DFT Study
}

\author{
Jörg J. Lippstreu and Bernd F. Straub* \\ Department Chemie und Biochemie \\ der Ludwig-Maximilians-Universität München, \\ Butenandtstr. 5-13 (Haus F), D-81377 München, Germany \\ E-mail: Bernd.F.Straubdcup.uni-muenchen.de
}

\section{Validation of the Used Methodology}

The used quantum-chemical methodology was validated both structurally and energetically. We compared Grubbs $\eta^{3}$-vinylcarbene complex $\mathbf{3 0}$ as well as first- and second-generation Grubbs catalysts with their non-simplified quantum-chemical models after completed geometry optimization at the B3LYP/LACVP* level of theory. The bond lengths of ruthenium $\sigma$-bonds differed by $0.01-0.03 \AA(\mathrm{Ru}=\mathrm{C})$ to 0.05 $0.07 \AA$ ( $\mathrm{Ru}-\mathrm{Cl}$ and $\mathrm{Ru}-\mathrm{P})$. Bond angles deviate generally by $1^{\circ}$ to $4^{\circ}$, cis-bond angles at ruthenium up to $6^{\circ}$. The experimental stability of structure $\mathbf{3 0}$ was reproduced theoretically by the predicted higher relative energies of alternative isomeric structures with a different vinylcarbene coordination mode.

The additional basis set flexibility in the single-point calculations generally provided only minor differences in relative energies. An exception is the alkyne insertion step with an overestimation of the Gibbs free reaction energy of about $-20 \mathrm{~kJ} \mathrm{~mol}{ }^{-1}$ at the B3LYP/LACVP* level of theory compared to the relative energies obtained at the triple-zeta single point level of theory. Since this step is clearly irreversible anyway, basis set effects are too small to affect the conclusions made in this study. 


\section{Energy Data of Model Complexes}

\begin{tabular}{|c|c|c|c|c|c|}
\hline$\#$ & $\begin{array}{c}E_{\text {total }} \\
(\mathrm{B} 3 \mathrm{LYP} / \mathrm{LACVP} \star) \\
{[\mathrm{a} \cdot \mathrm{u} \cdot]}\end{array}$ & $\begin{array}{c}\text { zero } \\
\text { point } \\
\text { energy } \\
{\left[\mathrm{kcal}^{-1}\right.} \\
\left.\mathrm{mol}^{-1}\right]\end{array}$ & 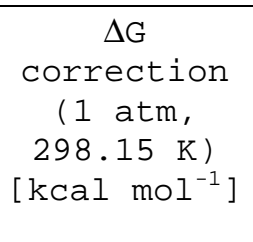 & $\begin{array}{c}E_{\text {total }} \text { single } \\
\text { point } \\
\text { (B3LYP / LACV3P**+ } \\
\text { / / } \\
\text { B3LYP / LACVP*) } \\
\text { [a.u. . }\end{array}$ & $\begin{array}{l}\mathrm{G}_{\text {relativ }} \\
{[\mathrm{kJ}} \\
\left.\mathrm{mol}^{-1}\right]\end{array}$ \\
\hline 1 & -1820.80898599054 & 186.348 & -31.8389 & -1821.0505596428 & 0.00 \\
\hline 2 & -1820.80351036481 & 185.906 & -31.8215 & -1821.0454076654 & 11.75 \\
\hline 3 & -1820.80655197381 & 186.144 & -32.2335 & -1821.0485781081 & 2.70 \\
\hline 4 & -1359.67797140408 & 113.816 & -25.8772 & -1359.8494754356 & 41.45 \\
\hline 5 & -1437.02078382863 & 132.508 & -27.801 & -1437.2204100741 & 53.72 \\
\hline $5 b$ & -1437.01700557720 & 132.023 & -28.2545 & -1437.2158343285 & 61.81 \\
\hline $5 c$ & -1437.00774403212 & 131.460 & -27.6761 & -1437.2072656970 & 84.37 \\
\hline 6 & -1436.99925233420 & 131.441 & -28.3510 & -1437.1982527331 & 105.13 \\
\hline 7 & -1437.08470091891 & 135.224 & -27.8845 & -1437.2778606425 & -86.10 \\
\hline 8 & -1437.07746709805 & 134.615 & -27.5964 & -1437.2715547072 & -70.89 \\
\hline 9 & -1437.08781170019 & 135.291 & -28.0525 & -1437.2810969742 & -95.02 \\
\hline 10 & -1898.21800270158 & 207.595 & -34.4819 & -1898.4811924313 & -136.79 \\
\hline 11 & -1515.68462386883 & 169.245 & -31.1882 & -1515.9030139988 & -62.71 \\
\hline 12 & -1515.68242986910 & 169.680 & -29.8372 & -1515.9011901635 & -50.45 \\
\hline 13 & -1515.69313266453 & 171.034 & -29.5749 & -1515.9111820756 & -69.92 \\
\hline 14 & -1515.67576761276 & 169.373 & -29.4724 & -1515.8943297496 & -32.20 \\
\hline 15 & -1515.67703531990 & 168.995 & -30.5923 & -1515.8954738829 & -41.47 \\
\hline 16 & -1438.27649782179 & 148.076 & -28.5190 & -1438.4736642797 & 71.14 \\
\hline $16 \mathrm{~b}$ & -1438.27542264353 & 148.231 & -28.2271 & -1438.4722500575 & 76.73 \\
\hline $16 c$ & -1438.27444712135 & 148.015 & -28.5150 & -1438.4720655149 & 75.10 \\
\hline 17 & -1438.27544335741 & 148.142 & -28.1646 & -1438.4724583287 & 76.07 \\
\hline 18 & -1438.29396858164 & 150.049 & -27.5881 & -1438.4897145715 & 41.15 \\
\hline 19 & -1514.43004540308 & 153.870 & -29.8382 & -1514.6511288672 & -80.28 \\
\hline 20 & -1514.40788769442 & 153.132 & -30.0054 & -1514.6282590094 & -24.02 \\
\hline 21 & -1514.49240934618 & 156.293 & -30.0257 & -1514.7077740373 & -219.64 \\
\hline 22 & -1515.66623459488 & 170.532 & -28.9813 & -1515.8824784466 & 5.82 \\
\hline 23 & -1515.69722565730 & 171.975 & -28.6769 & -1515.9132506259 & -67.66 \\
\hline 23 isomer & -1515.70026218678 & 172.243 & -28.2845 & -1515.9159988206 & -72.11 \\
\hline 24 & -1436.96679018513 & 132.268 & -28.6711 & -1437.1620699703 & 202.25 \\
\hline 25 & -1437.04608091480 & 135.144 & -27.2323 & -1437.2405333788 & 14.30 \\
\hline 26 & -1437.02996870700 & 134.128 & -27.2745 & -1437.2262660658 & 47.33 \\
\hline 27 & -1437.06140649924 & 134.897 & -28.0171 & -1437.2571085872 & -33.54 \\
\hline 28 & -1437.06037626873 & 134.517 & -27.8333 & -1437.2551069752 & -29.10 \\
\hline 29 & -1437.08240083031 & 134.786 & -28.6202 & -1437.2748894217 & -83.21 \\
\hline
\end{tabular}




\begin{tabular}{|c|c|c|c|c|c|}
\hline$\#$ & $\begin{array}{c}E_{\text {total }} \\
(\mathrm{B} 3 \mathrm{LYP} / \mathrm{LACVP} \star) \\
\text { [a.u. }]\end{array}$ & $\begin{array}{l}\text { zero } \\
\text { point } \\
\text { energy } \\
{\left[\mathrm{kcal}^{-1}\right]} \\
\left.\mathrm{mol}^{-1}\right]\end{array}$ & 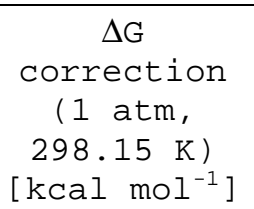 & $\begin{array}{c}E_{\text {total }} \text { single point } \\
\text { (B3LYP/LACV3P**+/ } \\
/ \\
\text { B3LYP / LACVP*) } \\
\text { [a.u. }]\end{array}$ & $\begin{array}{l}\mathrm{G}_{\text {relativ }} \\
{[\mathrm{kJ}} \\
\left.\mathrm{mol}^{-1}\right]\end{array}$ \\
\hline 30 & -2749.58373437400 & - & - & - & 0.00 \\
\hline 31 & -2749.57975149422 & - & - & - & 10.46 \\
\hline $\begin{array}{l}\text { 6-Me } \\
2,4-i \text { somer }\end{array}$ & -1515.64896829230 & 167.552 & -30.8783 & -1515.86800571300 & 105.84 \\
\hline $\begin{array}{l}\text { 6-Me } \\
2,3-i \text { somer }\end{array}$ & -1515.64517972774 & 167.926 & -30.4534 & -1515.86365933040 & 120.59 \\
\hline $\begin{array}{l}6-\mathrm{Me}_{1} \\
4-i \text { somer }\end{array}$ & -1476.32595375442 & 149.646 & -29.9565 & -1476.53461867241 & 108.66 \\
\hline $\begin{array}{l}\text { 6-Me }{ }_{1} \\
\text { 3-isomer }\end{array}$ & -1476.32390628683 & 149.995 & -29.6240 & -1476.53192527591 & 118.58 \\
\hline ethene & -78.58581299934 & 32.15 & -13.0998 & -78.6154688406 & - \\
\hline ethyne & -77.32383605858 & 16.645 & -11.9523 & -77.3563641018 & - \\
\hline propene & -117.90519708326 & 50.265 & -15.6864 & -117.94546243195 & - \\
\hline propyne & -116.65077344182 & 34.902 & -14.5650 & -116.69255106368 & - \\
\hline butadiene & -155.98884509371 & 53.656 & -16.1768 & -156.0406785745 & - \\
\hline $\mathrm{PMe}_{3}$ & -461.09394380789 & 71.176 & -17.7357 & -461.1643713828 & - \\
\hline
\end{tabular}




\begin{tabular}{|c|c|c|c|c|c|}
\hline$\#$ & $\begin{array}{c}E_{\text {total }} \\
(\mathrm{B} 3 \mathrm{LYP} / \mathrm{LACVP} \star) \\
{[\mathrm{a} \cdot \mathrm{u} \cdot]}\end{array}$ & $\begin{array}{l}\text { zero } \\
\text { point } \\
\text { energy } \\
{\left[\mathrm{kcal}^{-1}\right.} \\
\left.\mathrm{mol}^{-1}\right]\end{array}$ & $\begin{array}{c}\Delta \mathrm{G} \\
\text { correction } \\
(1 \mathrm{~atm}, \\
298.15 \mathrm{~K}) \\
{\left[\mathrm{kcal}^{-1}\right.} \\
\left.\mathrm{mol}^{-1}\right]\end{array}$ & $\begin{array}{c}E_{\text {total }} \text { single point } \\
\text { (B3LYP/LACV3P**+/ } \\
\text { /B3LYP/LACVP*) } \\
\text { [a.u.] }\end{array}$ & $\begin{array}{l}\mathrm{G}_{\text {relativ }} \\
{[\mathrm{kJ}} \\
\left.\mathrm{mol}^{-1}\right]\end{array}$ \\
\hline $1\left(1^{\text {st }}\right)$ & -1975.88847166297 & 162.2890 & -31.7578 & -1976.11468653858 & 0.00 \\
\hline $2\left(1^{\mathrm{st}}\right)$ & -1975.88813151471 & 162.0840 & -31.1178 & -1976.11437864389 & 2.63 \\
\hline $3\left(1^{s t}\right)$ & -1975.89215855923 & 162.4150 & -31.1959 & -1976.11907030806 & -8.63 \\
\hline $4\left(1^{\text {st }}\right)$ & -1514.75786228876 & 89.9580 & -25.0677 & -1514.91438177615 & 43.30 \\
\hline $5\left(1^{s t}\right)$ & -1592.09687307077 & 108.8050 & -26.7416 & -1592.28073849459 & 69.28 \\
\hline $5 b\left(1^{s t}\right)$ & -1592.10030819870 & 108.2360 & -27.2561 & -1592.28412159578 & 55.86 \\
\hline $5 c\left(1^{s t}\right)$ & -1592.09089607882 & 107.7760 & -26.9731 & -1592.27492127515 & 79.28 \\
\hline $6\left(1^{\mathrm{st}}\right)$ & -1592.06909939260 & 107.9520 & -27.7751 & -1592.25242775452 & 135.72 \\
\hline $7\left(1^{\mathrm{st}}\right)$ & -1592.16489995622 & 111.3390 & -27.1885 & -1592.34279478571 & -84.92 \\
\hline $8\left(1^{\text {st }}\right)$ & -1592.15736126352 & 110.7820 & -26.9518 & -1592.33605698379 & -68.57 \\
\hline $9\left(1^{s t}\right)$ & -1592.16695128820 & 111.4120 & -27.4037 & -1592.34504408304 & -91.42 \\
\hline $10\left(1^{\text {st }}\right)$ & -2053.29843334021 & 183.8840 & -34.0842 & -2053.54631284610 & -136.62 \\
\hline $11\left(1^{\text {st }}\right)$ & -1670.76336896567 & 145.5890 & -30.2758 & -1670.96650693468 & -55.88 \\
\hline $12\left(1^{\text {st }}\right)$ & -1670.75406429838 & 146.2490 & -28.8841 & -1670.95742465988 & -23.45 \\
\hline $13\left(1^{\text {st }}\right)$ & -1670.75947132179 & 147.2340 & -28.7037 & -1670.96248697646 & -31.87 \\
\hline $14\left(1^{\text {st }}\right)$ & -1670.74832510588 & 145.9170 & -28.6436 & -1670.95194669936 & -9.45 \\
\hline $15\left(1^{\text {st }}\right)$ & -1670.76014876362 & 145.3310 & -29.3265 & -1670.96392974099 & -46.22 \\
\hline $16\left(1^{\text {st }}\right)$ & -1593.35878853056 & 124.1680 & -27.8137 & -1593.54066516553 & 66.84 \\
\hline $16 \mathrm{~b}\left(1^{\text {st }}\right)$ & -1593.35950687144 & 124.4800 & -27.1981 & -1593.54119700386 & 69.33 \\
\hline $16 c\left(1^{\text {st }}\right)$ & -1593.35092413834 & 124.3790 & -27.3672 & -1593.53276072822 & 90.35 \\
\hline $17\left(1^{\text {st }}\right)$ & -1593.34718710783 & 124.2280 & -27.8966 & -1593.52834055031 & 99.10 \\
\hline $18\left(1^{\text {st }}\right)$ & -1593.36035920703 & 126.2680 & -26.9384 & -1593.54100557746 & 78.40 \\
\hline $19\left(1^{\text {st }}\right)$ & -1669.50710212785 & 130.1570 & -28.9977 & -1669.71272136109 & -69.00 \\
\hline $20\left(1^{\text {st }}\right)$ & -1669.47943128794 & 129.6160 & -28.8488 & -1669.68376043257 & 5.40 \\
\hline $21\left(1^{\text {st }}\right)$ & -1669.57180791306 & 132.5260 & -29.1468 & -1669.77163408490 & -214.38 \\
\hline $22\left(1^{s t}\right)$ & -1670.74296185315 & 146.8400 & -27.9684 & -1670.94391912250 & 18.31 \\
\hline $23\left(1^{\text {st }}\right)$ & -1670.77966449980 & 148.3110 & -27.6741 & -1670.97988019348 & -68.72 \\
\hline $24\left(1^{\text {st }}\right)$ & -1592.04508735929 & 108.6610 & -27.5695 & -1592.22482654014 & 231.64 \\
\hline $25\left(1^{s t}\right)$ & -1592.12170996184 & 111.4280 & -26.3389 & -1592.30079497634 & 48.91 \\
\hline $26\left(1^{s t}\right)$ & -1592.10159468225 & 110.3300 & -26.6369 & -1592.28245278121 & 91.23 \\
\hline $27\left(1^{\text {st }}\right)$ & -1592.13256789395 & 111.5190 & -26.4197 & -1592.31216114172 & 19.11 \\
\hline $28\left(1^{s t}\right)$ & -1592.13108351891 & 111.1080 & -26.3091 & -1592.30964330472 & 24.47 \\
\hline $29\left(1^{\text {st }}\right)$ & -1592.15854817675 & 111.2920 & -26.9657 & -1592.33676252696 & -48.71 \\
\hline $32\left(1^{s t}\right)$ & -1592.12216341698 & 111.0320 & -26.8438 & -1592.30147521896 & 23.72 \\
\hline $33\left(1^{\text {st }}\right)$ & -1592.12123640578 & 111.0870 & -26.0298 & -1592.30070024246 & 29.39 \\
\hline
\end{tabular}




\begin{tabular}{|c|c|c|c|c|c|}
\hline$\#$ & $\begin{array}{c}E_{\text {total }} \\
(\mathrm{B} 3 \mathrm{LYP} / \mathrm{LACVP} *) \\
{[\mathrm{a} \cdot \mathrm{u} \cdot]}\end{array}$ & $\begin{array}{l}\text { zero } \\
\text { point } \\
\text { energy } \\
{\left[\mathrm{kcal}^{-1}\right.} \\
\left.\mathrm{mol}^{-1}\right]\end{array}$ & $\begin{array}{c}\Delta \mathrm{G} \\
\text { correction } \\
(1 \mathrm{~atm}, \\
298.15 \mathrm{~K}) \\
{\left[\mathrm{kcal}^{-1}\right.} \\
\left.\mathrm{mol}^{-1}\right]\end{array}$ & $\begin{array}{c}\text { Etotal single point }_{\text {tB }} \\
\text { (B3LYP / LACV3P**+// } \\
\text { B3LYP / LACVP*) } \\
\text { [a.u. }]\end{array}$ & $\begin{array}{c}\text { G }_{\text {relativ }} \\
{\left[\mathrm{kJ} \mathrm{mol}^{-1}\right]}\end{array}$ \\
\hline 34 & -1632.35351418786 & 207.6940 & -34.2951 & -1632.61009056754 & 84.38 \\
\hline 35 & -1632.34952626776 & 207.5480 & -34.2735 & -1632.60573246362 & 91.58 \\
\hline 36 & -1632.34962941537 & 207.458 & -34.8388 & -1632.60610624379 & 95.31 \\
\hline 37 & -1632.36956129061 & 209.6740 & -33.3363 & -1632.62439919470 & 59.11 \\
\hline 37 isomer & -1632.36897627495 & 209.665 & -33.3701 & -1632.62379572879 & 60.51 \\
\hline 38 & -1632.35663720410 & 207.7720 & -33.8031 & -1632.61263299837 & 80.09 \\
\hline 39 & -1632.35616085137 & 207.7200 & -34.2407 & -1632.61229380984 & 78.94 \\
\hline 40 & -2014.89026978239 & 246.1380 & -37.6941 & -2015.19072498756 & 3.81 \\
\hline 41 & -1553.76253053123 & 173.6350 & -31.6506 & -1553.99292437271 & 37.11 \\
\hline 42 & -1553.76932896141 & 173.8140 & -30.2386 & -1553.99577270910 & 36.29 \\
\hline 43 & -1553.75163818300 & 174.0510 & -29.7885 & -1553.97672237394 & 89.18 \\
\hline 44 & -1553.82573558051 & 176.0010 & -30.2018 & -1554.04724387048 & -89.55 \\
\hline 45 & -1553.81746450152 & 175.5940 & -29.4339 & -1554.03968568985 & -68.19 \\
\hline 46 & -1553.82928855312 & 176.0770 & -30.1641 & -1554.05103092071 & -99.01 \\
\hline 47 & -2014.95382928290 & 248.3890 & -36.4520 & -2015.24557568513 & -125.58 \\
\hline 48 & -1632.42064235329 & 209.9930 & -33.3375 & -1632.66804574173 & -54.15 \\
\hline 49 & -1632.41790215646 & 210.4810 & -32.0919 & -1632.66521556836 & -39.47 \\
\hline 50 & -1632.42827706071 & 211.8270 & -31.6827 & -1632.67447210367 & -56.43 \\
\hline 51 & -1632.41190443332 & 210.2080 & -31.4973 & -1632.65855994932 & -20.65 \\
\hline 52 & -1632.41362902454 & 209.7740 & -32.6890 & -1632.66068931044 & -33.04 \\
\hline 48-Me & -1671.73906171882 & 227.868 & -33.8188 & -1671.99756190206 & $\begin{array}{c}(80.49 \text { to } \mathbf{4 7} \\
\left.+\mathrm{H}_{2} \mathrm{CCHMe}\right)\end{array}$ \\
\hline 49-Me & -1671.73179213884 & 228.240 & -32.9609 & -1671.98969027319 & $\begin{array}{c}(106.30 \text { to } 47 \\
\left.+\mathrm{H}_{2} \text { CCHMe }\right)\end{array}$ \\
\hline 50-Me & -1671.74338289830 & 229.476 & -32.6171 & -1672.00002184853 & $\begin{array}{r}(85.79 \text { to } \mathbf{4 7} \\
\left.+\mathrm{H}_{2} \mathrm{CCHMe}\right)\end{array}$ \\
\hline 51-Me & -1671.73303686978 & 227.959 & -33.0831 & -1671.99024192975 & $\begin{array}{r}(103.16 \text { to } 47 \\
\left.+\mathrm{H}_{2} \text { CCHMe }\right)\end{array}$ \\
\hline 48-OMe & -1746.94370779028 & 230.652 & -35.1732 & -1747.22668111048 & $\begin{array}{r}(78.70 \text { to } \mathbf{4 7} \\
\left.+\mathrm{H}_{2} \text { CCHOMe }\right)\end{array}$ \\
\hline 49-OMe & -1746.93495070713 & 231.054 & -33.8697 & -1747.21757762898 & $\begin{array}{c}(109.73 \text { to } 47 \\
+\mathrm{H}_{2} \text { CCHOMe) }\end{array}$ \\
\hline 50-OMe & -1746.94788157391 & 232.356 & -33.9420 & -1747.22950307572 & $\begin{array}{r}(83.57 \text { to } 47 \\
\left.+\mathrm{H}_{2} \mathrm{CCHOMe}\right)\end{array}$ \\
\hline 51-OMe & -1746.94450597064 & 231.525 & -34.2132 & -1747.22582690556 & $\begin{array}{r}(88.69 \text { to } \mathbf{4 7} \\
\left.+\mathrm{H}_{2} \text { CCHOMe }\right)\end{array}$ \\
\hline 53 & -1632.36100512177 & 207.6140 & -33.8030 & -1632.61682380962 & 68.43 \\
\hline 54 & -1632.34186012352 & 206.7400 & -34.4570 & -1632.59661420172 & 115.10 \\
\hline 55 & -1632.41618581518 & 209.7720 & -33.4764 & -1632.66624329565 & -50.93 \\
\hline 56 & -1632.41329186332 & 209.3760 & -33.1463 & -1632.66332767007 & -43.55 \\
\hline 57 & -1632.41734780096 & 210.1290 & -33.3865 & -1632.66737437887 & -52.03 \\
\hline 58 & -2093.54606215040 & 282.8320 & -39.0053 & -2093.86613761994 & -85.24 \\
\hline 59 & -1632.42938112073 & 211.0760 & -31.7745 & -1632.67629555649 & -64.74 \\
\hline 60 & -1632.41703583699 & 211.2140 & -30.9066 & -1632.66440931810 & -29.32 \\
\hline 61 & -1632.41940573088 & 211.8920 & -30.6546 & -1632.66595970335 & -29.50 \\
\hline 62 & -1632.40494239376 & 210.4090 & -30.5741 & -1632.65190472135 & 1.53 \\
\hline 63 & -1632.41068264879 & 209.5030 & -32.4332 & -1632.65845062266 & -27.23 \\
\hline $\mathrm{H}_{2} \mathrm{C}=\mathrm{CHOMe}$ & -193.10697760122 & 53.210 & -17.4656 & -193.17347990695 & \\
\hline $\begin{array}{l}\text { hept-1- } \\
\text { en-6-yne }\end{array}$ & -272.66522365250 & 92.4270 & -20.8088 & -272.75395413453 & - \\
\hline $\begin{array}{l}\text { 1-vinyl } \\
\text { cyclo } \\
\text { pentene }\end{array}$ & -272.72704965945 & 94.6300 & -19.2112 & -272.80710177523 & - \\
\hline
\end{tabular}




\section{Cartesian Coordinates of Model Complexes}

\section{Intermolecular Enyne Metathesis}

1

$\begin{array}{lrrr}\mathrm{C} & -0.4758870748 & 0.3400137769 & -2.4394668634 \\ \mathrm{~N} & -0.9214236553 & 0.6560441856 & -1.0710246635 \\ \mathrm{C} & -0.0834145410 & 0.1542587913 & -0.1456013644 \\ \mathrm{~N} & 0.9809945840 & -0.3787566817 & -0.7802551630 \\ \mathrm{C} & 0.9881910430 & -0.0557974218 & -2.2135371173 \\ \mathrm{Ru} & -0.1636766473 & 0.0940271287 & 1.9348409763 \\ \mathrm{P} & 0.4176764445 & -0.5256191671 & 4.1894055457 \\ \mathrm{C} & -0.9313683142 & -1.2484292883 & 5.2117878156 \\ \mathrm{C} & 2.2012536124 & -0.7936145950 & -0.1067978575 \\ \mathrm{C} & -2.2957135756 & 1.0676062467 & -0.8620750625 \\ \mathrm{C} & -1.5885514275 & 1.0884918881 & 2.4512910756 \\ \mathrm{C} 1 & 1.3862488810 & 2.0062425100 & 1.9805014119 \\ \mathrm{Cl} & -1.4511784617 & -2.0005181234 & 1.7902924617 \\ \mathrm{C} & 1.6766530369 & -1.8758432813 & 4.1366302332 \\ \mathrm{C} & 1.1826542181 & 0.7557763297 & 5.2653594370 \\ \mathrm{H} & 1.6760613652 & 0.7794126802 & -2.4106090465 \\ \mathrm{H} & 1.3015190451 & -0.9175225894 & -2.8108385147 \\ \mathrm{H} & -1.0723383202 & -0.4920598871 & -2.8414553291 \\ \mathrm{H} & -0.5948111229 & 1.2051546228 & -3.0987948031 \\ \mathrm{H} & 2.7358408720 & -1.5022602892 & -0.7475812360 \\ \mathrm{H} & 2.8455890429 & 0.0606322514 & 0.1310400914 \\ \mathrm{H} & 1.9453640450 & -1.3165673654 & 0.8201975777 \\ \mathrm{H} & -2.5111371483 & 1.9500873419 & -1.4750878103 \\ \mathrm{H} & -2.9927983513 & 0.2628097504 & -1.1337677168 \\ \mathrm{H} & -2.4358709551 & 1.3163980599 & 0.1886787027 \\ \mathrm{H} & -2.5746355738 & 0.6344852836 & 2.6157091553 \\ \mathrm{H} & -1.4906647895 & 2.1658160347 & 2.6354906814 \\ \mathrm{H} & -0.5413758130 & -1.6932403795 & 6.1341375039 \\ \mathrm{H} & -1.6498361571 & -0.4626734909 & 5.4662725622 \\ \mathrm{H} & -1.4443722252 & -2.0051263948 & 4.6116538268 \\ \mathrm{H} & 1.9438042455 & -2.2078165897 & 5.1463911070 \\ \mathrm{H} & 1.2606730378 & -2.7183122698 & 3.5755332008 \\ \mathrm{H} & 2.5804129963 & -1.5208959002 & 3.6306399471 \\ \mathrm{H} & 1.5584571752 & 0.3211385382 & 6.1985433131 \\ \mathrm{H} & 1.9921753180 & 1.2401719778 & 4.7133218748 \\ \mathrm{H} & 0.4358376859 & 1.5216716317 & 5.4962589581\end{array}$

2

$\begin{array}{rrrr}\mathrm{C} & 0.764597 & -0.497175 & -2.334128 \\ \mathrm{C} & -0.610985 & 0.169369 & -2.466296 \\ \mathrm{~N} & -0.902297 & 0.568053 & -1.079029 \\ \mathrm{C} & -0.030299 & 0.047500 & -0.187741 \\ \mathrm{~N} & 0.901344 & -0.638948 & -0.878329 \\ \mathrm{C} & -2.217143 & 1.113364 & -0.780398 \\ \mathrm{Ru} & -0.094094 & 0.209435 & 1.935027 \\ \mathrm{Cl} & -2.049818 & -1.244732 & 1.860476 \\ \mathrm{C} & 2.045700 & -1.362617 & -0.354731 \\ \mathrm{P} & -0.018031 & -0.034050 & 4.344287 \\ \mathrm{C} & 0.812090 & 1.301434 & 5.307285 \\ \mathrm{C} & -0.699502 & 1.920766 & 2.066652\end{array}$




\begin{tabular}{rrrr}
$\mathrm{Cl}$ & 2.263516 & 0.863899 & 2.113425 \\
$\mathrm{C}$ & -1.621246 & -0.269033 & 5.224499 \\
$\mathrm{C}$ & 0.946662 & -1.540627 & 4.795612 \\
$\mathrm{H}$ & 1.576667 & 0.130978 & -2.725986 \\
$\mathrm{H}$ & 0.820555 & -1.474075 & -2.827042 \\
$\mathrm{H}$ & -1.383241 & -0.527983 & -2.820394 \\
$\mathrm{H}$ & -0.604162 & 1.038367 & -3.132847 \\
$\mathrm{H}$ & 2.063330 & -2.370307 & -0.789330 \\
$\mathrm{H}$ & 2.982772 & -0.848314 & -0.599413 \\
$\mathrm{H}$ & 1.980648 & -1.434631 & 0.728766 \\
$\mathrm{H}$ & -2.251781 & 2.196040 & -0.958482 \\
$\mathrm{H}$ & -2.957561 & 0.633617 & -1.432510 \\
$\mathrm{H}$ & -2.482447 & 0.882632 & 0.249209 \\
$\mathrm{H}$ & -0.280937 & 2.637489 & 2.785387 \\
$\mathrm{H}$ & -1.487356 & 2.332462 & 1.425305 \\
$\mathrm{H}$ & -1.469572 & -0.534819 & 6.276784 \\
$\mathrm{H}$ & -2.202342 & 0.657348 & 5.167967 \\
$\mathrm{H}$ & -2.188705 & -1.051899 & 4.714591 \\
$\mathrm{H}$ & 1.005695 & -1.672053 & 5.882145 \\
$\mathrm{H}$ & 0.467908 & -2.420771 & 4.354308 \\
$\mathrm{H}$ & 1.955836 & -1.444237 & 4.384142 \\
$\mathrm{H}$ & 0.920771 & 1.024232 & 6.361752 \\
$\mathrm{H}$ & 1.794074 & 1.489538 & 4.865015 \\
$\mathrm{H}$ & 0.221660 & 2.221521 & 5.243744 \\
& & & \\
\hline & & &
\end{tabular}

$\begin{array}{lrrr}\mathrm{C} & -0.0197596250 & 0.0594176327 & -0.1993546541 \\ \mathrm{Ru} & -0.0087861739 & 0.1206853005 & 1.9328033293 \\ \mathrm{~N} & 0.9188697310 & -0.4473854969 & -1.0252252345 \\ \mathrm{~N} & -1.0225495331 & 0.5443570794 & -0.9804440707 \\ \mathrm{C} & 0.6487796115 & -0.1845466233 & -2.4450904985 \\ \mathrm{C} & 2.1820510974 & -1.0723331541 & -0.6755762941 \\ \mathrm{C} & -0.8104800789 & 0.2755384237 & -2.4120249755 \\ \mathrm{C} & -2.3915896372 & 0.8307082678 & -0.5746010767 \\ \mathrm{H} & 1.3282034068 & 0.5958707149 & -2.8167090888 \\ \mathrm{H} & 0.8067435627 & -1.0871612948 & -3.0450922849 \\ \mathrm{H} & -1.5041947349 & -0.5096472853 & -2.7465307087 \\ \mathrm{H} & -0.9961113548 & 1.1724560662 & -3.0125024104 \\ \mathrm{H} & 2.3098558889 & -1.9716255548 & -1.2910168023 \\ \mathrm{H} & 3.0253919957 & -0.3942850811 & -0.8543445711 \\ \mathrm{H} & 2.1855049893 & -1.3455716806 & 0.3757968279 \\ \mathrm{H} & -2.6940270070 & 1.8227963535 & -0.9340215691 \\ \mathrm{H} & -3.0682365616 & 0.0821301508 & -1.0083781153 \\ \mathrm{H} & -2.4813242757 & 0.7812597151 & 0.5064967220 \\ \mathrm{C} & -0.6876808659 & 1.8149750983 & 1.9231613383 \\ \mathrm{H} & -0.9959602672 & 2.3568349726 & 2.8265468487 \\ \mathrm{H} & -0.7786860435 & 2.3978329213 & 0.9995439235 \\ \mathrm{Cl} & 2.3526896572 & 0.5576122056 & 2.1504093728 \\ \mathrm{Cl} & -1.7819826748 & -1.5092780954 & 1.9081191095 \\ \mathrm{P} & -0.0954008716 & 0.0313300573 & 4.3582521719 \\ \mathrm{C} & 0.4884424864 & -1.5856028951 & 5.0273758695 \\ \mathrm{C} & 0.9006883278 & 1.2667945715 & 5.3004443016 \\ \mathrm{C} & -1.7825409624 & 0.1997832742 & 5.0899968784 \\ \mathrm{H} & -1.7716928566 & 0.0647747573 & 6.1774427032 \\ \mathrm{H} & -2.1853231518 & 1.1922815619 & 4.8609326109 \\ \mathrm{H} & -2.4344527341 & -0.5470769524 & 4.6284405342 \\ \mathrm{H} & 0.4035782057 & -1.6284090188 & 6.1192520203\end{array}$




$\begin{array}{rr}\mathrm{H} & -0.1113758645 \\ \mathrm{H} & 1.5342988165 \\ \mathrm{H} & 0.8388609965 \\ \mathrm{H} & 1.9403798824 \\ \mathrm{H} & 0.5392058820\end{array}$

4.5832571470

4. 7403032001

6.3815999153

4.9690927096

5.0756590336

$\begin{array}{lr}\mathrm{C} & 0.8223552058 \\ \mathrm{C} & -0.6425414418 \\ \mathrm{~N} & -1.0790240852 \\ \mathrm{C} & -0.0307276839 \\ \mathrm{~N} & 1.1078207799 \\ \mathrm{C} & -2.5022145174 \\ \mathrm{Ru} & -0.1230134205 \\ \mathrm{C} & -1.3697051207 \\ \mathrm{C} & 2.4203722887 \\ \mathrm{Cl} & -1.2179414306 \\ \mathrm{Cl} & 1.8150952294 \\ \mathrm{H} & 0.9624912403 \\ \mathrm{H} & 1.4953718978 \\ \mathrm{H} & -0.7497894472 \\ \mathrm{H} & -1.2474294504 \\ \mathrm{H} & 3.1865136654 \\ \mathrm{H} & 2.5414986517 \\ \mathrm{H} & 2.5549693201 \\ \mathrm{H} & -3.0215112489 \\ \mathrm{H} & -2.9070136546 \\ \mathrm{H} & -2.6824211509 \\ \mathrm{H} & -1.7328561995 \\ \mathrm{H} & -1.7723344326 \\ & \end{array}$

0.2854342274

$-0.1517446934$

$-0.0098872698$

0.0783432977

0.1539486395

$-0.0470546872$

0.1471201726

$-1.1548689738$

0.5784507405

2. 2375606537

$-1.0232193325$

1. 3298381384

$-0.3465800359$

$-1.1957404057$

0.4795619781

0.0710898863

1. 6645823135

0.2997638698

0.5967142099

$-1.0640326096$

0.3366058635

$-1.8179498968$

$-1.3430442550$
$-1.9832458965$

$-2.0623951303$

$-0.6674469424$

0.1871129802

$-0.5482020537$

$-0.3742473942$

2.1582419362

2. 3298994892

$-0.0817982505$

2.1065182490

2.8559553438

$-2.2988391035$

$-2.5704174053$

$-2.3901512775$

$-2.7205971957$

$-0.6770306611$

$-0.2029480732$

0.9610755106

$-1.0931006086$

$-0.4684882730$

0.6277455608

1. 5395649213

3. 3366113289

5

$\begin{array}{lr}\mathrm{C} & -0.0759905389 \\ \mathrm{~N} & 0.9303078067 \\ \mathrm{C} & 0.5659794011 \\ \mathrm{C} & -0.9613201187 \\ \mathrm{~N} & -1.1629661755 \\ \mathrm{C} & 2.3372355473 \\ \mathrm{C} & -2.5088852048 \\ \mathrm{Ru} & -0.1876077228 \\ \mathrm{Cl} & -1.3665264067 \\ \mathrm{Cl} & 1.1423973998 \\ \mathrm{C} & 1.3208907545 \\ \mathrm{C} & -1.5325510462 \\ \mathrm{C} & -0.8822141401 \\ \mathrm{H} & 1.0274781187 \\ \mathrm{H} & 0.9137470002 \\ \mathrm{H} & -1.4560797742 \\ \mathrm{H} & -1.3741205114 \\ \mathrm{H} & 2.8160557148 \\ \mathrm{H} & 2.4347193802 \\ \mathrm{H} & 2.8287662699 \\ \mathrm{H} & -3.0571716067 \\ \mathrm{H} & -3.0339308752 \\ \mathrm{H} & -2.4678631043 \\ & \end{array}$

0.0292696908

$-0.2755823894$

$-0.0031417023$

0.0992123106

0.3542569407

$-0.3912158875$

0.5236374555

0.1432897717

$-2.0298029357$

2. 2032727578

$-0.7475566686$

0.2801569603

1. 3427446119

0.9403738123

$-0.8075024931$

$-0.8401037992$

0.9124377933

0.5942649497

$-0.8702450612$

$-1.0146974942$

1.2066055169

$-0.4353535414$

0.9726401636
0.1351571045

$-0.6981445038$

$-2.1008478591$

$-2.0206774560$

$-0.5868535679$

$-0.3569392362$

$-0.0608545737$

2. 2147824975

1.9930911116

1.8384440695

2. 6918289208

3. 9078465287

3. 8781423091

$-2.4247066309$

$-2.7553725381$

$-2.3059475029$

$-2.6248966851$

$-0.3160626443$

0.6166240923

$-1.1103468690$

$-0.7176281282$

0.0100959766

0.9341120128 


$\begin{array}{rrrr}\mathrm{H} & 2.2494682814 & -0.2028697972 & 2.8871849862 \\ \mathrm{H} & 1.2922870010 & -1.8237496113 & 2.8862123891 \\ \mathrm{H} & -2.2064526833 & -0.5024560584 & 4.1978982209 \\ \mathrm{H} & -0.4927923038 & 2.3144372825 & 4.1118046033\end{array}$

$5 b$

$\begin{array}{lrrr}\mathrm{C} & 0.6351959267 & 0.0150859048 & -2.2397967475 \\ \mathrm{C} & -0.8795159387 & -0.2166417983 & -2.1993206343 \\ \mathrm{~N} & -1.1913375884 & 0.0382843146 & -0.7884975414 \\ \mathrm{C} & -0.0945394268 & 0.0714785864 & -0.0066356664 \\ \mathrm{~N} & 0.9966877612 & 0.0172136674 & -0.8131696327 \\ \mathrm{C} & -2.5843039844 & 0.1401721463 & -0.3873927495 \\ \mathrm{Ru} & -0.1289298286 & 0.1977987531 & 2.0348615930 \\ \mathrm{C} & 1.3848587229 & -0.7845463176 & 2.2349220087 \\ \mathrm{C} & 2.3565897799 & 0.4212331435 & -0.4837594825 \\ \mathrm{Cl} & -1.6977277120 & -1.6583543587 & 2.1221069717 \\ \mathrm{Cl} & 0.4533837663 & 2.5168692641 & 1.6950933900 \\ \mathrm{H} & 0.8981918503 & 0.9843986157 & -2.6867922118 \\ \mathrm{H} & 1.1759018885 & -0.7689715140 & -2.7799050214 \\ \mathrm{H} & -1.1541080939 & -1.2471977480 & -2.4633993083 \\ \mathrm{H} & -1.4381832229 & 0.4649309071 & -2.8493748310 \\ \mathrm{H} & 2.6430004152 & 1.2679085195 & -1.1211334977 \\ \mathrm{H} & 2.4103386628 & 0.7461178298 & 0.5522682064 \\ \mathrm{H} & 3.0617791753 & -0.4004291276 & -0.6611733550 \\ \mathrm{H} & -3.0908144309 & 0.8287260302 & -1.0748668488 \\ \mathrm{H} & -3.0818664417 & -0.8348350964 & -0.4121708654 \\ \mathrm{H} & -2.6561526799 & 0.5344059463 & 0.6241890508 \\ \mathrm{H} & 1.8380471388 & -0.8716647267 & 3.2300588132 \\ \mathrm{H} & 1.8592413644 & -1.3728566408 & 1.4433689998 \\ \mathrm{C} & 0.0875840694 & 1.0131679044 & 4.3899129843 \\ \mathrm{C} & -0.5871985714 & 0.0038201117 & 4.4853542807 \\ \mathrm{H} & 0.6425852062 & 1.9255066916 & 4.3844934193 \\ \mathrm{H} & -1.1854366065 & -0.8667496741 & 4.6482093649\end{array}$

$5 c$

$\mathrm{N}$

C

C

$\mathrm{N}$

C

$\mathrm{C}$

$\mathrm{Ru}$

Cl

C

$\mathrm{Cl}$

C

$\mathrm{H}$

$\mathrm{H}$

$\mathrm{H}$
1.0907709209

0.8251114912

$-0.6844875334$

$-1.0947969620$

$-0.0539963499$

$-2.5176476681$

$-0.1420988689$

0.7716087389

2.4395392122

1.1724250663

$-2.0493070109$

0.0975092844

$-0.8547490896$

1.1115992974

1.4047418615

$-0.9393886960$

$-1.1976973667$

2. 7267314989

2.4827773347

3.1502654545
$-0.2190550352$

$-0.2156306449$

$-0.4510196154$

$-0.1227304512$

$-0.0922900104$

$-0.0082866683$

0.1186939033

2.3073550652

0.1649712154

$-1.1289804561$

$-1.2955418369$

0.3452825482

1. 0060710887

0.7580923044

$-0.9954443747$

$-1.4968482943$

0.1908084277

1.0866272486

0.3504027164

$-0.6274003271$
$-0.6678932225$

$-2.1149529275$

$-2.1708762737$

$-0.7999565807$

0.0617950590

$-0.5252672608$

2.0755347899

1. 8251311334

$-0.2683217738$

2. 2882301024

2.4005055859

4.7663711188

4. 4274596331

$-2.5400939957$

$-2.6208321520$

$-2.3966947116$

$-2.8950495302$

$-0.7879299733$

0.7989619289

$-0.5420687487$ 


$\begin{array}{rr}\mathrm{H} & -2.9599782468 \\ \mathrm{H} & -3.0121925131 \\ \mathrm{H} & -2.6803955990 \\ \mathrm{H} & 1.6022474889 \\ \mathrm{H} & 1.5501626614 \\ \mathrm{H} & -1.6896219507 \\ \mathrm{H} & 0.9199389969\end{array}$

0.6613186664
-0.9821685722
0.3952093754
-1.3574774153
-1.7499764425
1.6362498387
-0.2250867709

$-1.2709992084$

$-0.5799096745$

0.4670084389

3. 2692823842

1. 4749555847

4. 2084748088

5.1278256360

0.2659356294

0.0872369805

0.4689492211

0.4728291025

0.6111766966

$-0.0235653166$

0.6454959792

0.0747800921

0.1417925104

$-0.1827378116$

2.4199798461

$-0.5164018315$

$-2.1917031394$

1. 4645063352

$-0.2492654774$

$-0.4718262333$

1. 3024675944

0.9580647769

$-0.4501825593$

$-0.6871270870$

1. 2627526408

$-0.3600423459$

1. 1236464464

0.1872808023

$-1.5862312190$

0.4001142318

$-0.4262400023$
0.1025513404

$-0.9034278150$

$-2.1978790451$

$-1.8743633896$

$-0.4121435646$

$-0.7868114075$

0.3641811794

2.1167514245

4.1722482453

4. 5132667109

2.2400050409

2. 5881663316

1. 9204303879

$-2.4930626742$

$-2.9766180244$

$-2.1591753434$

$-2.3393870703$

$-0.8811286364$

0.1837305034

$-1.5730266498$

$-0.1622802189$

0.5309719279

1.3301947837

2.7892867450

2.6903789911

4. 3213106661

5.0795028072
C

C

$\mathrm{N}$

C

$\mathrm{N}$

C

$\mathrm{Ru}$

$\mathrm{Cl}$

C

$\mathrm{Cl}$

C

$\mathrm{H}$

$\mathrm{H}$

$\mathrm{H}$

$\mathrm{H}$

$\mathrm{H}$

$\mathrm{H}$
0.9517690091

$-0.4513739734$

$-0.9565264673$

0.0408757062

1. 1860312387

$-2.2749175150$

$-0.1427337661$

1. 3262483244

2. 5376425096

$-2.3214033529$

0.8164816698

0.9927346998

1.7153231624

$-0.4273483983$

$-1.0978433764$

3. 2234549840

2. 8465967103
0.2752991732

$-0.3296633246$

$-0.1812979830$

0.0739974689

0.2621293139

$-0.7227669610$

0.1163937061

$-1.7176845365$

0.4870674048

0.9868018391

1. 6711909339

1. 3052241495

$-0.3138053606$

$-1.3936183466$

0.1971924778

$-0.1545196797$

1. 5316717552
$-2.2296387587$

$-2.3336620307$

$-0.9629993479$

$-0.0774372336$

$-0.7805171557$

$-0.6677134022$

1.8876989676

2.1595254497

$-0.2948498164$

2. 2708518392

2.1044425295

$-2.6124068813$

$-2.7474726216$

$-2.6119626513$

$-3.0424209060$

$-0.8600510126$

$-0.4392299074$ 


$\begin{array}{rr}\mathrm{H} & 2.6023335642 \\ \mathrm{H} & -2.9977911585 \\ \mathrm{H} & -2.2758103650 \\ \mathrm{H} & -2.5763745689 \\ \mathrm{C} & 1.0879799174 \\ \mathrm{H} & 1.2577979038 \\ \mathrm{C} & 0.2718037314 \\ \mathrm{H} & 2.0271227303 \\ \mathrm{H} & 0.5494850360 \\ \mathrm{H} & -0.7037708366\end{array}$

0.2159940006

$-0.3017096276$

$-1.8172813362$

$-0.4441383507$

2.2361257883

2. 2008093473

2. 1483046062

2.7870351859

2.5930830944

1.6747804059
0.7563884964

$-1.3747502820$

$-0.7742293743$

0.3395464773

3.4154897067

1.2537632120

4.4859681634

3. 5137825062

5.4375067521

4.4205661944 $\begin{array}{rr}0.2061622565 & -0.7552463609 \\ 0.1514765955 & -2.2090430742 \\ -0.5049555153 & -2.3189563496 \\ -0.2993807977 & -0.9726094843 \\ 0.0307429978 & -0.0756685261 \\ -0.8863260504 & -0.6788985521 \\ 0.1829403539 & 1.8782110374 \\ 1.6730400130 & 2.0887082655 \\ 2.0575056466 & 3.4609739390 \\ 2.9929240965 & 4.1866062873 \\ 0.5105010259 & -0.2418788145 \\ -1.6549862377 & 2.3023106983 \\ 1.1174154839 & 2.0517908571 \\ 1.1656849843 & -2.6338573989 \\ -0.4312882648 & -2.6838170600 \\ -1.5811234947 & -2.5366855538 \\ -0.0394799936 & -3.0733783576 \\ -0.0911877774 & -0.7928294420 \\ 1.5707721816 & -0.3783059076 \\ 0.2430894016 & 0.8112581144 \\ -0.5415143367 & -1.4269673779 \\ -1.9838427648 & -0.7196575577 \\ -0.5620683842 & 0.3015310435 \\ 2.3149508766 & 1.2543520672 \\ 1.4948924516 & 3.9019055176 \\ 3.2181194010 & 5.2027838405 \\ 3.5433908208 & 3.7946772008\end{array}$

9

C

C

$\mathrm{N}$

C

$\mathrm{N}$

C

$\mathrm{Ru}$

$\mathrm{Cl}$

C

$\mathrm{Cl}$

C

$\mathrm{H}$

$\mathrm{H}$

$\mathrm{H}$

\begin{abstract}
1.1221374703
$-0.2157972708$

$-0.8398569341$

0.0702403418

1.2505519589

$-2.1259550529$

$-0.2733007369$

1.2501496502

2. 5376855702

$-2.5087084478$

0.6171247779

1. 1042574984

1.9664239028

$-0.0837943126$
\end{abstract}

0.1113214497

$-0.6197850754$

$-0.3731916810$

0.0267951321

0.2342513321

$-1.0018276874$

0.2049057644

$-1.5277295693$

0.6157097979

0.9920366267

1. 8061741114

1.1072176458

$-0.4478966592$

$-1.7009560441$
$-2.1608509278$

$-2.3005204821$

$-0.9942607494$

$-0.0679843840$

$-0.7032221757$

$-0.7297383818$

1. 8644622893

2.3858739069

$-0.1454105797$

2.0134728887

2.0366911341

$-2.6267831003$

$-2.5758392381$

$-2.4558008241$ 


$\begin{array}{lr}\mathrm{H} & -0.8413361284 \\ \mathrm{H} & 3.3166078837 \\ \mathrm{H} & 2.7642515427 \\ \mathrm{H} & 2.5468518942 \\ \mathrm{H} & -2.8178727991 \\ \mathrm{H} & -2.0232930211 \\ \mathrm{H} & -2.5356669418 \\ \mathrm{C} & 0.8748331946 \\ \mathrm{H} & 0.9463232862 \\ \mathrm{C} & 1.4885069607 \\ \mathrm{H} & 0.5611925042 \\ \mathrm{H} & 1.6768129431 \\ \mathrm{H} & 1.8141578439\end{array}$

\section{0}

$$
\begin{aligned}
& \text { C } \\
& \text { N } \\
& \text { C } \\
& \text { C } \\
& \text { N } \\
& \text { C } \\
& \text { C }
\end{aligned}
$$

0.0507356000

0.6414720748

0.1669429007

$-0.4601062000$

$-0.6934593803$

1. 3117970981

$-1.2991136775$

$\mathrm{Ru}$

C

$\mathrm{H}$

$\mathrm{Cl}$

Cl

$\mathrm{P}$

C

C

C

$\mathrm{H}$

$\mathrm{H}$

$\mathrm{H}$

$\mathrm{H}$

$\mathrm{H}$

$\mathrm{H}$

$\mathrm{H}$

$\mathrm{H}$

C

$\mathrm{H}$

$\mathrm{H}$

$\mathrm{H}$

$\mathrm{H}$

$\mathrm{H}$

$\mathrm{H}$

$\mathrm{H}$

C

$\mathrm{H}$

$\mathrm{H}$

$\mathrm{H}$

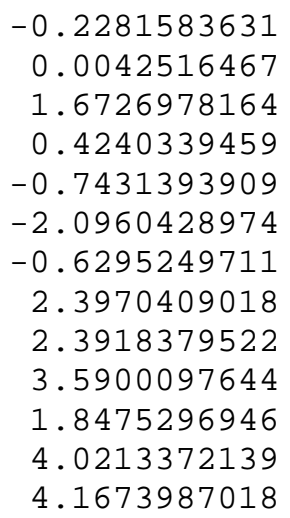

$-3.1084467417$

$-0.6151077158$

$-0.3430316855$

0.9253151689

$-1.5380806116$

$-0.6906591115$

0.2070779372

3.3307698870

1.1706874731

3. 4762002846

4. 2180372371

4. 4552003091

2. 6136801082

$\begin{array}{rr}-0.1957014410 & -0.2244026892 \\ 0.3725064847 & -1.2926498860 \\ -0.2173862358 & -2.5565872099 \\ -1.5317941641 & -2.0761308553 \\ -1.2343422219 & -0.6570020003 \\ 1.6579675218 & -1.3257683729 \\ -2.2157605602 & 0.2274043259 \\ 0.2006801374 & 1.8172695151 \\ 1.7015460434 & 2.0489496526 \\ 2.6758276337 & 2.0162476226 \\ -1.5616595241 & 2.0338344760 \\ 1.5546542358 & 1.5507621263 \\ 0.0335645235 & 4.1723054614 \\ 1.5435840094 & 4.9837749022 \\ -1.2179069683 & 4.4366949985 \\ -0.5243195848 & 5.2909633411 \\ 0.4475100826 & -3.0232912013 \\ -0.3617284809 & -3.2558094401 \\ -2.3803411762 & -2.1791454074 \\ -1.7773428704 & -2.5867663286 \\ 2.4608725176 & -1.6142599542 \\ 1.8765670827 & -0.3418673562 \\ 1.6192066783 & -2.0513791581 \\ -2.7063435444 & -0.2976457536 \\ -2.9650124314 & 0.5643839418 \\ -1.7017237437 & 1.0966757835 \\ 1.7659833431 & 2.3015835125 \\ 1.3262516767 & 5.9885960754 \\ 2.2959941863 & 5.0535882809 \\ 1.9436914364 & 4.3532332487 \\ -1.3082483208 & 5.4986437446 \\ -0.9101565002 & 3.8771311818 \\ -2.1924288475 & 4.0668871555 \\ -1.7141262897 & 6.3044855284 \\ 0.2475669253 & 5.3288268271 \\ 2.9458026048 & 2.4706700936 \\ 0.8283027394 & 2.3447754224 \\ 3.0014187814 & 2.6562667272 \\ -8900814988 & 2.4267995549\end{array}$ 


$\begin{array}{lrrr}\mathrm{N} & -0.7749295438 & -0.7346004130 & -0.8357989510 \\ \mathrm{C} & 0.0557452688 & 0.0233314012 & -0.0888436157 \\ \mathrm{~N} & 0.9193083948 & 0.6503586438 & -0.9127239069 \\ \mathrm{C} & 0.5628889798 & 0.4344990090 & -2.3263938051 \\ \mathrm{C} & -0.3508345856 & -0.7929732075 & -2.2401644822 \\ \mathrm{Ru} & -0.2150907644 & 0.0303544610 & 1.9287302995 \\ \mathrm{Cl} & -1.6345046135 & 2.0116503893 & 1.7223089451 \\ \mathrm{C} & 1.7498671994 & 1.7921904481 & -0.5737132168 \\ \mathrm{C} & -1.6886562468 & -1.7243808103 & -0.2854159011 \\ \mathrm{C} & -1.4876387089 & -0.5998182924 & 3.7792394550 \\ \mathrm{C} & -0.5106948842 & 0.1348109797 & 4.3972728378 \\ \mathrm{C} & 1.1785406321 & 1.0612070257 & 2.5942047843 \\ \mathrm{C} & 2.5168509360 & 0.6351259748 & 2.9661087028 \\ \mathrm{C} & 3.4164089950 & 1.5213214757 & 3.4405558908 \\ \mathrm{Cl} & 0.9077817539 & -2.1581889535 & 2.0839098056 \\ \mathrm{H} & 0.0320899872 & 1.3157997834 & -2.7154423828 \\ \mathrm{H} & 1.4581049834 & 0.2679676764 & -2.9327405559 \\ \mathrm{H} & 0.1951234022 & -1.7278056206 & -2.4340609457 \\ \mathrm{H} & -1.2111789749 & -0.7406416621 & -2.9140548013 \\ \mathrm{H} & 1.1878062890 & 2.7325630519 & -0.6504768265 \\ \mathrm{H} & 2.1265806628 & 1.6837309252 & 0.4414164471 \\ \mathrm{H} & 2.6021929553 & 1.8246697848 & -1.2601609752 \\ \mathrm{H} & -2.4798834036 & -1.9173293726 & -1.0165608256 \\ \mathrm{H} & -1.1735166790 & -2.6564324171 & -0.0284183585 \\ \mathrm{H} & -2.1668489092 & -1.3210161481 & 0.6137237278 \\ \mathrm{H} & 0.9698273041 & 2.1296479431 & 2.7531183464 \\ \mathrm{H} & 2.7703069910 & -0.4134865238 & 2.8412042331 \\ \mathrm{H} & 4.4261995737 & 1.2224343317 & 3.7105365476 \\ \mathrm{H} & 3.1652929846 & 2.5728338976 & 3.5690806447 \\ \mathrm{H} & -0.6663270848 & 1.1861116849 & 4.6180942549 \\ \mathrm{H} & -1.4044868835 & -1.6816665387 & 3.7254879356 \\ \mathrm{H} & -2.4374471867 & -0.1354484731 & 3.5273555469 \\ \mathrm{H} & 0.3630271179 & -0.3505142671 & 4.8203110294 \\ & & & \end{array}$

12

$\begin{array}{lrrr}\mathrm{N} & -0.8521807154 & -0.8450391981 & 0.6125966881 \\ \mathrm{C} & 0.0237457653 & -0.1325459823 & -0.1195391176 \\ \mathrm{~N} & 0.9064838455 & 0.4563593996 & 0.7048795475 \\ \mathrm{C} & 0.7253398031 & 0.0016965897 & 2.0948624005 \\ \mathrm{C} & -0.6890013035 & -0.5929571599 & 2.0513198846 \\ \mathrm{Ru} & -0.1413271458 & -0.0686706472 & -2.1622982954 \\ \mathrm{Cl} & -1.8715604607 & 1.6604464328 & -1.9038287164 \\ \mathrm{C} & 2.1602735834 & 1.0723942930 & 0.3081879058 \\ \mathrm{C} & -2.0828459466 & -1.4074067086 & 0.0773103694 \\ \mathrm{C} & 1.1334087712 & 0.9738596446 & -3.0659924331 \\ \mathrm{H} & 2.0604724582 & 0.4668036641 & -3.3611772441 \\ \mathrm{C} & -0.2403922726 & 0.0748647161 & -4.5204969638 \\ \mathrm{C} & -1.1757913000 & -0.7975990316 & -3.9246631269 \\ \mathrm{C} 1 & 1.3487196624 & -2.0042463844 & -2.3011338633 \\ \mathrm{H} & 0.8228817023 & 0.8371269292 & 2.7943573623 \\ \mathrm{H} & 1.4836499686 & -0.7557080045 & 2.3409423545 \\ \mathrm{H} & -0.7876873562 & -1.5185163425 & 2.6262368066 \\ \mathrm{H} & -1.4478784804 & 0.1230313661 & 2.3985430032 \\ \mathrm{H} & 2.0517345806 & 1.5044683664 & -0.6854043648 \\ \mathrm{H} & 2.9771859662 & 0.3384573038 & 0.2945293105\end{array}$




$\begin{array}{rr}\mathrm{H} & 2.4059316595 \\ \mathrm{H} & -2.4029373717 \\ \mathrm{H} & -1.8904499687 \\ \mathrm{H} & -2.8771721060 \\ \mathrm{C} & 1.1236565023 \\ \mathrm{H} & -2.2029909557 \\ \mathrm{H} & -0.9878463108 \\ \mathrm{H} & 0.6240629762 \\ \mathrm{H} & -0.5765164089 \\ \mathrm{C} & 2.2326379205 \\ \mathrm{H} & 0.2077336718 \\ \mathrm{H} & 2.2527794029 \\ \mathrm{H} & 3.1558772143\end{array}$

13

C

$\mathrm{N}$

C

C

$\mathrm{N}$

C

C

$\mathrm{Ru}$

C

C

C

C

C

$\mathrm{Cl}$

Cl

$\mathrm{H}$

$\mathrm{H}$

$\mathrm{H}$

$\mathrm{H}$

$\mathrm{H}$

$\mathrm{H}$

$\mathrm{H}$

$\mathrm{H}$

$\mathrm{H}$

$\mathrm{H}$

$\mathrm{H}$

$\mathrm{H}$

$\mathrm{H}$

$\mathrm{H}$

$\mathrm{H}$

$\mathrm{H}$

$\mathrm{H}$

$\mathrm{H}$

14

$\begin{array}{lr}\mathrm{N} & 0.8513941139 \\ \mathrm{C} & 0.5816037338 \\ \mathrm{C} & -0.8634906040 \\ \mathrm{~N} & -0.9861474772 \\ \mathrm{C} & -0.0282673996\end{array}$

$-0.0799570514$

0.7625145360

0.4955971135

$-0.9261519178$

$-1.0164111968$

2.0391046850

$-2.2453070579$

0.0464517545

1.1033493686

0.2084157144

$-0.9942410905$

$-1.0718135655$

$-2.2419466216$

1.7570610839

$-1.5697505207$

0.5658752675

1.2238940657

$-1.0760401160$

$-1.6908997691$

1.9564694280

2.8407929702

2.2787071592

$-2.6031761586$

$-2.0434802302$

$-3.0162414457$

2.1601823936

0.8780307355

$-0.2866447023$

$-1.9422127198$

0.8463093468

$-0.1331836900$

$-2.2828333150$

$-3.1926214766$
1.8712779182
-2.2362944760
-1.8138562345
-0.6546867541
2.4184158084
-0.4596538605
-1.8674719725
-0.3524185665
1.0506415673
3.0684594857
2.9547750936
4.1502717445
2.5369871430
1.0156782082

0.7162343538

$-0.9207029114$

0.0108395025

$-3.2666833484$

$-3.8148711915$

$-3.9588074338$

$-5.0197767948$

$-4.8555882646$

$-3.6659680844$

$-3.0360080436$

$-3.7690072253$

$-3.8896861644$ $\begin{array}{rr}-0.2009539570 & -0.1280319837 \\ 0.3799509844 & 0.7372907935 \\ -0.0679755384 & 2.1138537045 \\ -0.6371298389 & 1.9859268128 \\ -0.8933252945 & 0.5386852382 \\ 0.9785787550 & 0.3767864975 \\ -1.4025228509 & -0.0544458533 \\ -0.0896564826 & -2.1424406410 \\ 0.9571258250 & -3.4478579733 \\ 0.0826510844 & -4.4086599488 \\ -0.7205193388 & -3.7387000680 \\ -2.1679810091 & -3.9781555441 \\ -2.8121605750 & -4.1183944550 \\ -1.8290627296 & -2.1031678723 \\ 1.7456026272 & -1.9795346938 \\ 0.7671506095 & 2.8166854320 \\ -0.8386993453 & 2.4037796891 \\ -1.5586952378 & 2.5555402731 \\ 0.0940338147 & 2.2842693531 \\ 1.4244518754 & -0.6159459479 \\ 0.2301471403 & 0.3644046828 \\ 1.7677035453 & 1.0964768883 \\ -2.2453213304 & 0.5449235848 \\ -1.7623210269 & -1.0654348734 \\ -0.6237466146 & -0.1002647427 \\ 0.6969790349 & -3.5035268629 \\ 2.0222112977 & -3.4882291755 \\ 0.7864270387 & -5.0816409099 \\ -0.1913297146 & -3.8451576162 \\ -0.6322699736 & -4.9283906140 \\ -2.7157078581 & -4.0021879742 \\ -3.8865297551 & -4.2735991850 \\ -2.2832845251 & -4.0847172674\end{array}$
0.3452550085

$-0.1196244189$

$-0.6137672341$

$-0.8319003220$

$-0.1748978825$
0.7632567630

2.1345858394

2.0201027473

0.5705895647

$-0.1130822495$ 


$\begin{array}{lr}\mathrm{C} & -2.2503187173 \\ \mathrm{Ru} & 0.0089596199 \\ \mathrm{C} & 0.2761267755 \\ \mathrm{C} & -0.8851524621 \\ \mathrm{C} & 2.1736475605 \\ \mathrm{Cl} & 1.8949275780 \\ \mathrm{Cl} & -1.8910167113 \\ \mathrm{C} & 1.0984545811 \\ \mathrm{H} & 0.7073534272 \\ \mathrm{H} & 1.2756939223 \\ \mathrm{H} & -1.0521086322 \\ \mathrm{H} & -1.5879703737 \\ \mathrm{H} & 2.1288430604 \\ \mathrm{H} & 2.9047138834 \\ \mathrm{H} & 2.4834943978 \\ \mathrm{H} & -2.5911452137 \\ \mathrm{H} & -2.1122466088 \\ \mathrm{H} & -3.0087676724 \\ \mathrm{H} & 2.1639150865 \\ \mathrm{H} & 0.7047555926 \\ \mathrm{H} & 0.1672740453 \\ \mathrm{H} & -1.8310990640 \\ \mathrm{C} & -0.9594402234 \\ \mathrm{H} & 1.1958527782 \\ \mathrm{C} & -2.1239361335 \\ \mathrm{H} & -0.0220638788 \\ \mathrm{H} & -2.1611163153 \\ \mathrm{H} & -3.0701339601 \\ & \end{array}$

-1.3003971955
-0.0855535857
-0.0206128947
-0.6957718918
0.8610863031
-1.6641903817
1.4709407868
1.2602948452
0.6939421957
-0.9318021071
-1.5380520745
0.1469752190
1.4143265084
0.0506881995
1.5412932113
-2.1564810107
-1.6301832372
-0.5094959016
1.0927329438
2.2621063042
0.9488261433
-0.1631572879
-2.1483860203
-0.5683350622
-2.7863223331
-2.6982496167
-3.8636718926
-2.2502882146
0.0217790001

$-2.1697243341$

$-4.5397662521$

$-4.1360692265$

0.4441769977

$-2.1359339763$

$-2.0547568821$

$-2.7907356707$

2.8551067918

2.3951199105

2.5743523539

2. 3456368301

$-0.4930848752$

0.3390975072

1. 2436846854

0.6139142662

$-1.0089348998$

0.0363509689

$-2.9823152328$

$-2.9930000324$

$-5.0163527480$

$-4.2021405312$

$-3.9438265372$

$-4.7192722674$

$-3.7309942521$

$-3.9606395311$

$-3.5993839054$

$-3.6964004420$
C

$\mathrm{N}$

C

C

$\mathrm{N}$

C

$\mathrm{Ru}$

C

C

C

C

$\mathrm{C}$

$\mathrm{Cl}$

$\mathrm{Cl}$

$\mathrm{H}$

$\mathrm{H}$

$\mathrm{H}$

$\mathrm{H}$

$\mathrm{H}$

$\mathrm{H}$

$\mathrm{H}$

$\mathrm{H}$

$\mathrm{H}$
$-0.0393226142$

0.8452194331

0.5581680730

$-0.8904310277$

$-1.0148990649$

2.1829010506

$-2.2901463717$

0.0179744574

1.0412624096

0.3452781382

$-0.8325211051$

$-0.9291092751$

$-2.0953121074$

1. 9695151124

$-1.9918530974$

0.6868706042

1. 2411059806

$-1.0904908338$

$-1.6097672672$

2. 1419448853

2.8597194838

2.5561711545

$-2.6017464833$

$-2.1850296383$

$-3.0556888684$

2. 1130332562

0.6020033391
$-0.1783851913$

0.2925592293

$-0.2119214070$

$-0.6855423499$

$-0.8384801336$

0.7897379144

$-1.2756396173$

$-0.0593372616$

1. 4263304599

$-0.1224741277$

$-0.7311504701$

$-2.1445320838$

$-2.7248104088$

$-1.5641358786$

1. 3591680185

0.5773891115

$-1.0391501116$

$-1.6317315831$

0.0660136355

1. 5140395034

$-0.0223747263$

1. 2963175414

$-2.1842011481$

$-1.5049761779$

$-0.4995042701$

1. 3450933641

2. 4309602308
$-0.1515554605$

0.7504708558

2.1041647682

1. 9637986319

0.5068106593

0.4658094084

$-0.0415913440$

$-2.2037029670$

$-2.4638707171$

$-4.6618680729$

$-4.2993545473$

$-3.9208178039$

$-3.5853919052$

$-2.1589339790$

$-2.1382212230$

2. 8506600587

2.3454047303

2.4755353883

2. 3207101884

$-0.3484878232$

0.1793836403

1. 3607732777

0.4860239429

$-1.1007016218$

0.0687227071

$-2.6838916759$

$-2.4778903928$ 


$\begin{array}{lr}\mathrm{H} & 0.3280876013 \\ \mathrm{H} & -1.7639996715 \\ \mathrm{H} & 1.2824304118 \\ \mathrm{H} & -0.0032301247 \\ \mathrm{H} & -2.1456087360 \\ \mathrm{H} & -3.0290280937\end{array}$

16

$$
\text { C }
$$

$\mathrm{N}$

C

$\mathrm{N}$

C

Ru

Cl

C

C

C

Cl

$\mathrm{H}$

$\mathrm{H}$

$\mathrm{H}$

$\mathrm{H}$

$\mathrm{H}$

$\mathrm{H}$

$\mathrm{H}$

$\mathrm{H}$

$\mathrm{H}$

$\mathrm{H}$

$\mathrm{H}$

$\mathrm{H}$

C

C

$\mathrm{H}$

$\mathrm{H}$

$\mathrm{H}$

$\mathrm{H}$

$16 b$

$\begin{array}{lr}\mathrm{N} & 1.0244528009 \\ \mathrm{C} & 0.6859227861 \\ \mathrm{C} & -0.8277352510 \\ \mathrm{~N} & -1.1631005915 \\ \mathrm{C} & -0.0798381953 \\ \mathrm{C} & -2.5626639029 \\ \mathrm{Ru} & -0.1266866458 \\ \mathrm{Cl} & 0.3823525375 \\ \mathrm{C} & 2.3742927401 \\ \mathrm{C} & 1.3871698033 \\ \mathrm{C} 1 & -1.6849369149 \\ \mathrm{C} & -0.6481569136 \\ \mathrm{C} & 0.2182731937 \\ \mathrm{H} & 0.9502770263 \\ \mathrm{H} & 1.2399159000 \\ \mathrm{H} & -1.0921376626\end{array}$

0.8785759594
-0.1784399860
-0.6689423035
-2.7140456221
-3.7754827128
-2.1666250651

$$
\begin{aligned}
& -5.0803756318 \\
& -4.3914170171 \\
& -4.6903145778 \\
& -3.9022705604 \\
& -3.3147206378 \\
& -3.5857208536
\end{aligned}
$$

0.0237346069

$-0.2799258954$

0.0768016463

0.4726755320

0.3157707530

$-0.0020043259$

1.0797842913

0.5605362905

$-0.7952835303$

1. 5204047654

$-1.8910795682$

1. 2215620344

$-0.5193747597$

$-0.8240912467$

0.8932970113

1. 5603303329

0.3262478514

$-0.1738918210$

$-1.6349414984$

$-1.1442999007$

$-0.0257176934$

1. 8809999111

2. 1722994872

0.0243715392

$-0.8874065864$

1. 0459719805

$-0.6180665527$

$-1.9311023330$

$-0.2601021334$
2.2951621524

0.8893229949

0.0570791479

0.8172702867

2.2570786224

$-1.9835621299$

$-2.0946326570$

0.4152127213

0.5523501814

$-2.0964498713$

$-1.8928836834$

2.8064766155

2.6487859648

2.9379150097

2.6188921809

0.6242827840

$-0.6412158531$

0.9851498510

1.2218151502

$-0.4773095165$

0.6632089148

$-3.0410675792$

$-1.2357476812$

$-4.4985326795$

$-4.2320425777$

$-4.7592649040$

$-4.2655555947$

$-4.0655633548$

$-4.5506243606$
0.0499908637

0.0365430247

$-0.2034668355$

0.0610276746

0.1062538146

0.1620921566

0.2441171976

2. 5674003279

0.4734946922

$-0.7428989234$

$-1.6163998481$

0.0334221977

1.0733676776

1.0046856256

$-0.7478148934$

$-1.2378975162$
$-0.8045785671$

$-2.2371157826$

$-2.2185106373$

$-0.8142877542$

$-0.0169154949$

$-0.4368695274$

2.0396602373

1. 6213199713

$-0.4568888437$

2. 2313572227

2.0823479484

4. 4528356114

4.3583696701

$-2.6857447925$

$-2.7630309830$

$-2.4775470019$ 


$\begin{array}{lr}\mathrm{H} & -1.3801313319 \\ \mathrm{H} & 2.6586110134 \\ \mathrm{H} & 2.4087686540 \\ \mathrm{H} & 3.0928174487 \\ \mathrm{H} & -3.0596247623 \\ \mathrm{H} & -3.0566581642 \\ \mathrm{H} & -2.6514343499 \\ \mathrm{H} & 1.8858372068 \\ \mathrm{H} & 1.8279192173 \\ \mathrm{H} & 1.2878474788 \\ \mathrm{H} & -0.3224583833 \\ \mathrm{H} & -0.1261629283 \\ \mathrm{H} & -1.7226973521\end{array}$

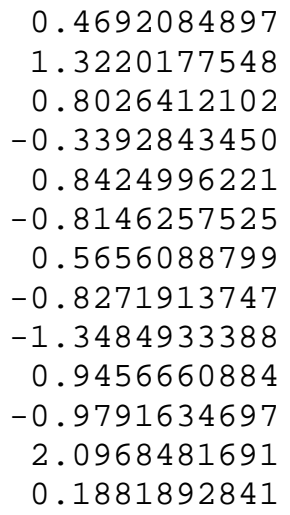

$16 c$

\begin{tabular}{|c|c|}
\hline $\mathrm{N}$ & 0.8115657937 \\
\hline $\mathrm{C}$ & 0.3098150998 \\
\hline $\mathrm{C}$ & -1.2073709828 \\
\hline $\mathrm{N}$ & -1.3272881096 \\
\hline $\mathrm{C}$ & -0.1571963101 \\
\hline $\mathrm{C}$ & -2.6310041548 \\
\hline $\mathrm{Ru}$ & -0.1343810623 \\
\hline $\mathrm{Cl}$ & 0.7394801620 \\
\hline $\mathrm{C}$ & 2.2427986363 \\
\hline $\mathrm{C}$ & 1.5250656992 \\
\hline $\mathrm{Cl}$ & -1.0030311616 \\
\hline $\mathrm{C}$ & -1.1939220400 \\
\hline $\mathrm{C}$ & -0.6539272286 \\
\hline $\mathrm{H}$ & 0.6170479919 \\
\hline $\mathrm{H}$ & 0.7072164507 \\
\hline $\mathrm{H}$ & -1.5926910517 \\
\hline $\mathrm{H}$ & -1.7679327558 \\
\hline $\mathrm{H}$ & 2.5842020734 \\
\hline $\mathrm{H}$ & 2.4810011409 \\
\hline $\mathrm{H}$ & 2.7579197307 \\
\hline $\mathrm{H}$ & -3.3364084192 \\
\hline $\mathrm{H}$ & -2.9908115407 \\
\hline $\mathrm{H}$ & -2.5713661354 \\
\hline $\mathrm{H}$ & 1.7016161712 \\
\hline $\mathrm{H}$ & 2.3710250714 \\
\hline $\mathrm{H}$ & -1.2426593846 \\
\hline $\mathrm{H}$ & -0.6894303219 \\
\hline $\mathrm{H}$ & 0.3073827777 \\
\hline $\mathrm{H}$ & -2.2305718092 \\
\hline
\end{tabular}

$$
\begin{array}{r}
-0.0396693613 \\
0.2074742065 \\
0.1065521878 \\
0.3308820567 \\
0.1327437011 \\
0.3276345619 \\
0.2024743820 \\
2.4804102019 \\
0.0217520435 \\
-0.4194530731 \\
-2.1097459838 \\
-0.0118202049 \\
1.2522790383 \\
1.2097760027 \\
-0.5324794625 \\
-0.8899952064 \\
0.8594739671 \\
1.0590041549 \\
-0.5192593906 \\
-0.4623285155 \\
0.8745342289 \\
-0.6912501401 \\
0.8555527674 \\
-1.4915661627 \\
0.2668155888 \\
2.1108351118 \\
-0.8416044397 \\
1.4840709399 \\
-0.1930234019
\end{array}
$$

$-2.8830152391$

$-1.0927780617$

0.5785426094

$-0.6204189514$

$-1.1393643351$

$-0.4607182035$

0.5695349080

3.2057371959

1. 4334242478

4. 4958251354

4. 6652474612

4. 2620531564

4.4386925308
$-0.8925087492$

$-2.2573324825$

$-2.0618120399$

$-0.6140619929$

0.0197526431

0.0316646322

2.0918380961

1. 6759088709

$-0.6502251096$

2. 4514802889

1. 9314002239

4.1393090862

4.0957396186

$-2.5877836417$

$-2.9577241811$

$-2.3208364288$

$-2.6235162448$

$-0.5512538690$

0.2656461947

$-1.4852536506$

$-0.6014812101$

0.2109761284

0.9885116057

2. 5949143632

2. 5719111903

3. 7869352952

4. 6216180148

4.5411206813

3.8690070428

17

C

C

$\mathrm{N}$

C

$\mathrm{N}$

C

$\mathrm{Ru}$

$\mathrm{Cl}$

C
0.6208772053

$-0.8610260845$

$-1.0306879615$

$-0.0340835520$

0.9030127200

$-2.3340563331$

$-0.0348245740$

1. 5956292572

2. 2617763480
-0.2375018187
-0.6189029591
-0.7542192142
-0.1512074669
0.2593305067
-1.0889911430
-0.0392680633
-1.8600795592
0.6531363884

2.1225325564

2. 0203752790

0.5656365056

$-0.1091328283$

0.7640731907

0.0106953892

$-2.1565622863$

$-2.1986801338$

0.4238451209 


$\begin{array}{lrrr}\mathrm{Cl} & -1.7945322529 & 1.6612483520 & -2.0876394065 \\ \mathrm{C} & 1.1651608221 & 1.1880381183 & -2.8313032696 \\ \mathrm{C} & -0.8828627211 & -0.7356756222 & -4.0670763744 \\ \mathrm{C} & 0.1367980528 & 0.0862601552 & -4.5612241630 \\ \mathrm{H} & 0.8197881393 & 0.5352782272 & 2.8707629889 \\ \mathrm{H} & 1.2563608854 & -1.1084575000 & 2.3376096107 \\ \mathrm{H} & -1.1044968152 & -1.5542331239 & 2.5332708415 \\ \mathrm{H} & -1.5200032917 & 0.1746682658 & 2.4014868684 \\ \mathrm{H} & 2.2483929927 & 1.2411929835 & -0.4934338365 \\ \mathrm{H} & 2.9031461256 & -0.2235638253 & 0.2716079592 \\ \mathrm{H} & 2.6610113572 & 1.2701120183 & 1.2349928510 \\ \mathrm{H} & -2.7274019723 & -1.9664471017 & 0.5350041238 \\ \mathrm{H} & -2.2365195527 & -1.3369140365 & -1.0482004545 \\ \mathrm{H} & -3.0346446408 & -0.2508079402 & 0.1008467832 \\ \mathrm{H} & 2.2044001270 & 0.9133127375 & -3.0413323970 \\ \mathrm{H} & 0.8682875646 & 2.2256505407 & -3.0130353558 \\ \mathrm{H} & -0.1169114571 & 1.0641154478 & -4.9595451091 \\ \mathrm{H} & -1.9075405703 & -0.3755753424 & -4.0802998445 \\ \mathrm{H} & -0.7090134280 & -1.8084338667 & -4.0238956590 \\ \mathrm{H} & 1.0808747857 & -0.3566212368 & -4.8609895643\end{array}$

18

C

$\mathrm{N}$

C

$\mathrm{C}$

$\mathrm{N}$

C

C

$\mathrm{Ru}$

C

C

$\mathrm{Cl}$

$\mathrm{Cl}$

$\mathrm{H}$

$\mathrm{H}$

$\mathrm{H}$

$\mathrm{H}$

$\mathrm{H}$

$\mathrm{H}$

$\mathrm{H}$

$\mathrm{H}$

$\mathrm{H}$

$\mathrm{H}$

$\mathrm{H}$

$\mathrm{H}$

$\mathrm{H}$

19

C

C
0.0000000000

0.5669516485

0.5380997429

$-0.5380997429$

$-0.5669516485$

1.5098683828

$-1.5098683828$

0.0000000000

0.5234690936

0.0000000000

$-0.5234690936$

2.2540860569

$-2.2540860569$

0.2864136259

1.5223712832

$-0.2864136259$

$-1.5223712832$

1.2568839904

2.5375576263

1.4327949384

$-1.4327949384$

$-1.2568839904$

$-2.5375576263$

1.5942679754

$-0.1085409289$

$-0.8430821157$

$-1.5942679754$

0.1085409289

0.8430821157
0.0000000000

0.9406086246

0.5484151932

$-0.5484151932$

$-0.9406086246$

1. 9431430884

$-1.9431430884$

0.0000000000

1.2401953405

0.0000000000

$-1.2401953405$

$-0.9235700264$

0.9235700264

1.4016972915

0.1607768874

$-1.4016972915$

$-0.1607768874$

2.2208932369

1.5605929682

2. 8329196407

$-2.8329196407$

$-2.2208932369$

$-1.5605929682$

1.4082433249

2. 1220220717

0.3555345151

$-1.4082433249$

$-2.1220220717$

$-0.3555345151$
$-1.3149168052$

$-2.0837029531$

$-3.5027674631$

$-3.5027674631$

$-2.0837029531$

$-1.6090902764$

$-1.6090902764$

0.7080789311

2.1553479644

2.9890879365

2. 1553479644

0.6536420993

0.6536420993

$-4.1390756525$

$-3.8015934865$

$-4.1390756525$

$-3.8015934865$

$-0.5846791483$

$-1.6265921075$

$-2.2418909672$

$-2.2418909672$

$-0.5846791483$

$-1.6265921075$

2.2648488343

2.2558543515

3. 5827618679

2.2648488343

2.2558543515

3.5827618679

0.5744920923

0.0933630497

$-2.0329963120$

$-0.7222815984$

$-0.7096883364$

$-1.8852669341$ 


$\begin{array}{lr}\mathrm{N} & -1.0066353459 \\ \mathrm{C} & 0.0813815078 \\ \mathrm{~N} & 1.0630092739 \\ \mathrm{C} & -2.1993102698 \\ \mathrm{Ru} & -0.0514690997 \\ \mathrm{C} & 1.7057425570 \\ \mathrm{H} & 2.0110050414 \\ \mathrm{C} & 2.2847754149 \\ \mathrm{C} & -1.2465308485 \\ \mathrm{C} & -1.0961272168 \\ \mathrm{C} I & 0.2265310947 \\ \mathrm{Cl} & -0.2983801392 \\ \mathrm{H} & 0.3886399045 \\ \mathrm{H} & 1.3089572344 \\ \mathrm{H} & -0.5788264578 \\ \mathrm{H} & -1.5441243921 \\ \mathrm{H} & 2.7313773879 \\ \mathrm{H} & 2.9867763510 \\ \mathrm{H} & 2.0858641849 \\ \mathrm{H} & -2.0866288053 \\ \mathrm{H} & -2.3990220231 \\ \mathrm{H} & -3.0529644763 \\ \mathrm{C} & 2.6943111394 \\ \mathrm{H} & -1.5271361175 \\ \mathrm{H} & -1.1288263375 \\ \mathrm{C} & 3.8904012667 \\ \mathrm{H} & 2.4516613523 \\ \mathrm{H} & 4.6438182807 \\ \mathrm{H} & 4.1444712781 \\ & \end{array}$

\section{0}

$\mathrm{N}$

C

$\mathrm{N}$

C

C

$\mathrm{Ru}$

Cl

C

C

Cl

C

C

C

C

C

$\mathrm{H}$

$\mathrm{H}$

$\mathrm{H}$

$\mathrm{H}$

$$
\begin{array}{r}
-0.5409944131 \\
-0.1412568597 \\
0.1409348296 \\
-1.1356144629 \\
-0.0023132117 \\
0.3307945996 \\
1.3849969996 \\
0.8756546629 \\
0.6275328468 \\
-0.6040207105 \\
-2.4794053967 \\
2.4101060314 \\
1.1146020587 \\
-0.3844874805 \\
-1.7751748992 \\
-0.3249478694 \\
0.5001766751 \\
0.7082902510 \\
1.9488104967 \\
-2.2179620968 \\
-0.6883792421 \\
-0.9187151684 \\
-0.6002009292 \\
1.6474435679 \\
-1.6157445122 \\
-0.1626864918 \\
-1.6577871801 \\
-0.8488618432 \\
0.8956249135
\end{array}
$$

-0.4536460354
0.2293274953
-0.6428010008
0.1288709875
2.3060518027
2.7608012133
2.7510615842
-0.3560125138
4.0080506441
4.1173699901
2.1598450951
1.7650288545
-2.3949021631
-2.6875005209
-2.1153978447
-2.4964736168
0.5637288396
-1.1781290918
-0.2507700942
0.2561698448
1.1058438569
-0.5215071026
3.2638524517
4.1850356830
4.4719635078
3.7123862258
3.2677733466
4.0901296667
3.7170934264

$$
\begin{array}{rrr}
-1.0349580800 & -0.8001810908 & -0.5120148967 \\
-0.0126186424 & -0.1975359986 & 0.1214943678 \\
0.9122375988 & 0.1670959253 & -0.7867321871 \\
0.4203867768 & -0.0453312902 & -2.1594907914 \\
-0.7391434790 & -1.0229940212 & -1.9338851780 \\
-0.0298050263 & 0.0892792995 & 2.1469173444 \\
0.5515338717 & -2.3043069153 & 2.3548283985 \\
1.9895807171 & 1.1162496012 & -0.5563916834 \\
-2.0963331775 & -1.5404626350 & 0.1541870234 \\
-0.8653868382 & 2.3461661506 & 1.7564640608 \\
1.5455550374 & 0.7804792562 & 2.9073505298 \\
2.7187297639 & 0.0496840583 & 3.3620312155 \\
3.8587401074 & 0.6989063064 & 3.6671151686 \\
-0.9852068523 & -0.1414740859 & 4.0525778043 \\
0.0083554888 & 0.4878871381 & 4.4775088362 \\
0.0760005191 & 0.9087419872 & -2.5840896091 \\
1.2100020852 & -0.4506605696 & -2.7989272852 \\
-0.4373068083 & -2.0676851890 & -2.0972451554 \\
-1.6140944031 & -0.8078201097 & -2.5545075742 \\
2.4113658794 & 0.9520034710 & 0.4344385658 \\
2.7729271336 & 0.9431449427 & -1.3009922776 \\
1.6340077475 & 2.1517934309 & -0.6299468697 \\
-1.7857802710 & -2.5625255754 & 0.3972247020 \\
-2.3782441547 & -1.0262504598 & 1.0774000015 \\
-2.9748158156 & -1.5587251728 & -0.4987435250 \\
1.6270130822 & 1.8749033245 & 2.9353112547
\end{array}
$$




$\begin{array}{rrrr}\mathrm{H} & 0.6072456631 & 1.0304148267 & 5.1805880097 \\ \mathrm{H} & -1.9193287650 & -0.6531169035 & 4.1920027028 \\ \mathrm{H} & 2.6518914758 & -1.0329936479 & 3.4089087041 \\ \mathrm{H} & 4.7558868488 & 0.1637157579 & 3.9667985463 \\ \mathrm{H} & 3.9293335146 & 1.7838714810 & 3.6135504242\end{array}$

21

$\mathrm{N}$

C

C

$\mathrm{N}$

$\mathrm{Ru}$

Cl

$\mathrm{C}$

C

C

Cl

$\mathrm{H}$

$\mathrm{H}$

$\mathrm{H}$

$\mathrm{H}$

$\mathrm{H}$

$\mathrm{H}$

$\mathrm{H}$

$\mathrm{H}$

22

C

C

C

$\mathrm{N}$

C

C

$\mathrm{Ru}$

$\mathrm{Cl}$

Cl

$\mathrm{C}$

C

C

C
-0.3299118439000
-1.4833386345000
-2.1131344871000
-1.5001133949000
-0.4299768338000
-1.7953102485000
0.7653446629000
1.1518441860000
0.5997341370000
-0.5941078913000
-0.3699681591000
0.6905782609000
0.8739376650000
1.8917581031000
1.6584540474000
-2.1576793938000
-1.1626869425000
-1.8506194317000
-3.2043747779000
1.4767126764000
0.9047497223000
0.1377193136000
-1.6742060923000
-1.1099543737000
-2.8304830774000
1.4219247168000
-1.1347260310000
-1.6295666865000
0.1388975433000
2.0060899353000
2.6386454029000
-0.4159571402000
-1.0212781823000
-1.8488360176000
-1.2687175383000
-0.4814609424000
-1.9289724116000
0.4039128893000
2.5224697529000
0.3681407741000
0.8405321982000
1.2618685408000
0.8890643528000
1.3194307698000
0.9037816518000
-1.6434017797000
-0.2339468690000
-1.6248310756000
-2.9145531223000
-1.7666435401000
0.6278231540000
-0.2312095879000
1.2993098522000
-3.0141882012000
-1.5905356883000
-1.7231367124000
0.1921795668000
1.9038125582000
0.8962866548000
2.0161909269000
1.2471807830000
0.2060775909000
$-1.2298651003000$

$-1.9033789750000$

$-0.7797756563000$

0.4229099829000

0.1197437974000

1.6878689783000

1. 4104413473000

0.4090765559000

$-2.0263635376000$

2. 5868781147000

3. 9442284713000

4.7186354349000

6.0867080760000

6.8630766826000

2.2075936604000

$-2.2708613714000$

$-2.7585491296000$

$-0.8500166236000$

$-0.7421887557000$

$-1.4383599904000$

$-2.8920400513000$

$-2.3757337632000$

1. 5719025307000

2.4598968708000

1. 9873251836000

4. 3100451936000

4.3899140427000

2. 2312558671000

6.4897518710000

7.8868781960000

6.4915681940000

$$
\begin{array}{r}
-0.1394114297 \\
0.7783355757 \\
0.5256082993 \\
-0.9041813534 \\
-1.0442029597 \\
2.0491726049 \\
-2.2880103375 \\
-0.2005376049 \\
1.5215928797 \\
-1.8578750446 \\
0.8682400593 \\
-0.1424175164 \\
-1.1698735782 \\
-0.9107686639 \\
-1.8652642318
\end{array}
$$
$-0.3406274077$
0.3526998149
0.3000699892
$-0.2585933586$
$-0.8519033628$
0.8560109225
$-1.4949555933$
$-0.7690765516$
$-2.3492050977$
1.1238554694
0.6583468854
0.6877591695
$-0.4018878511$
$-1.7617949851$
$-2.7114682721$

-0.1765528297
0.5128143859
1.9614843214
2.0180817383
0.6827005066
0.0252751993
0.2974019596
-2.1644293876
-2.0074584606
-1.6876827089
-3.1992701742
-4.3709363516
-4.0451770389
-4.5240246086
-4.6448278999 


$\begin{array}{lr}\mathrm{H} & 0.6169793789 \\ \mathrm{H} & 1.2562540799 \\ \mathrm{H} & -1.0451531639 \\ \mathrm{H} & -1.6514781794 \\ \mathrm{H} & 2.1468458439 \\ \mathrm{H} & 2.8708571786 \\ \mathrm{H} & 2.1090229100 \\ \mathrm{H} & -3.0944317008 \\ \mathrm{H} & -2.5553010225 \\ \mathrm{H} & -2.1504742164 \\ \mathrm{H} & -2.2150532532 \\ \mathrm{H} & 0.3410835211 \\ \mathrm{H} & 0.9316816850 \\ \mathrm{H} & 1.8609246717 \\ \mathrm{H} & -0.6485071901 \\ \mathrm{H} & 0.1085067333 \\ \mathrm{H} & -1.6334338494 \\ \mathrm{H} & -2.9009617639\end{array}$

1.2946241593

$-0.3675892968$

$-1.0106809932$

0.5368359909

0.6146574970

0.3801412440

1.9417237009

$-0.7617294059$

$-2.2338097289$

$-2.0144318673$

$-0.1134133389$

0.4899873511

1. 6176180707

0.2786247333

1. 6547846651

$-2.0106961920$

$-3.7041861692$

$-2.5104762912$
2.4088749136

2.4399623993

2.8001238779

2.1511904950

$-1.0298648353$

0.5759746898

0.1671030902

0.1807899109

1.0604804166

$-0.6535526433$

$-4.0018405563$

$-5.3394039149$

$-2.6830982007$

$-3.4599104956$

$-4.4153301684$

$-4.8191716698$

$-5.0189627679$

$-4.3794973527$

\section{3}

$\mathrm{N}$

C

$\mathrm{N}$

C

C

$\mathrm{Ru}$

C

C

C

C

$\mathrm{C}$

$\mathrm{Cl}$

$\mathrm{Cl}$

C

$\mathrm{H}$

$\mathrm{H}$

$\mathrm{H}$

$\mathrm{H}$

$\mathrm{H}$

$\mathrm{H}$

$\mathrm{H}$

$\mathrm{H}$

$\mathrm{H}$

$\mathrm{H}$

$\mathrm{H}$

$$
\begin{array}{r}
-1.0080399887 \\
-0.1484040160 \\
0.8265726032 \\
0.6805009402 \\
-0.7280158700 \\
-0.4797887553 \\
-0.9922040266 \\
-0.5613396548 \\
-1.2673043083 \\
2.0914846696 \\
-2.2753867928 \\
1.4326030141 \\
-2.2797889886 \\
0.8289927915 \\
0.0531066532 \\
0.7842568874 \\
1.4609560356 \\
-0.7731556599 \\
-1.4698985724 \\
2.2248639106 \\
2.9082519475 \\
2.1346936575 \\
-3.1024165559 \\
-2.4551909308 \\
-2.2269857638 \\
-2.0474181608 \\
0.6650266525 \\
0.8889174753 \\
1.7985082816 \\
-0.4247048527 \\
0.4490450288 \\
-0.8442564872 \\
-2.3443145808
\end{array}
$$

0.7297896408

$-0.1867161240$

0.4418497514

1. 9080808030

2. 0787210726

$-2.2628758050$

$-4.4226586682$

$-4.3399786924$

$-3.4425888789$

$-0.1327907852$

0.4503264161

$-1.9686735541$

$-1.8856552945$

$-3.2571943493$

$-4.5525797492$

2. 3443512650

2. 3317037247

2. 8202238031

2.3474963747

$-1.1038927316$

0.5208515538

$-0.2233000111$

0.4391434304

1.2119597923

$-0.5243087726$

$-4.3952660482$

$-5.4607702649$

$-2.5866552588$

$-3.3894284067$

$-4.5519790402$

$-4.6275881729$

$-3.1823459293$

$-3.3243471620$ 
more stable conformer of 23

$\begin{array}{lrrr}\mathrm{C} & 0.0794561846 & -0.1973323478 & 0.0912602092 \\ \mathrm{~N} & 1.1917447348 & -0.6797156947 & -0.5060911314 \\ \mathrm{C} & 1.1306719779 & -0.5620190148 & -1.9728805276 \\ \mathrm{C} & -0.3437783942 & -0.2154545840 & -2.2121613075 \\ \mathrm{~N} & -0.7907608270 & 0.1664392296 & -0.8650544112 \\ \mathrm{C} & 2.5019783943 & -0.8323363242 & 0.1059672729 \\ \mathrm{C} & -2.1227678412 & 0.7235398008 & -0.6934421320 \\ \mathrm{Ru} & -0.3103873649 & -0.0256771704 & 2.1534579435 \\ \mathrm{Cl} & -2.4095582340 & -1.2393893806 & 1.9243304421 \\ \mathrm{Cl} & 1.2028556896 & 1.8755414062 & 1.9356270729 \\ \mathrm{C} & 0.4790965061 & -2.0125638824 & 2.4457483616 \\ \mathrm{C} & 1.3773440300 & -1.7127630929 & 3.6459550074 \\ \mathrm{C} & 0.8120529639 & -0.3591082935 & 4.0072591234 \\ \mathrm{C} & -0.5677394448 & -0.2693567379 & 4.3656920790 \\ \mathrm{C} & -1.2389598152 & 0.9075033990 & 4.0516618208 \\ \mathrm{H} & 1.8074329339 & 0.2379418425 & -2.3054049826 \\ \mathrm{H} & 1.4379543243 & -1.4956672688 & -2.4549924012 \\ \mathrm{H} & -0.9252367117 & -1.0778040236 & -2.5665002660 \\ \mathrm{H} & -0.4862754241 & 0.6098962356 & -2.9173589250 \\ \mathrm{H} & 2.8828452831 & -1.8503749258 & -0.0418373558 \\ \mathrm{H} & 3.2044245359 & -0.1238792225 & -0.3521198335 \\ \mathrm{H} & 2.4385801636 & -0.6027030292 & 1.1663137323 \\ \mathrm{H} & -2.2527676517 & 1.5420020041 & -1.4116197324 \\ \mathrm{H} & -2.8962846095 & -0.0349766676 & -0.8552262683 \\ \mathrm{H} & -2.2361802336 & 1.1120427310 & 0.3170221052 \\ \mathrm{H} & 1.4688792273 & 0.4909109093 & 4.1695537597 \\ \mathrm{H} & 1.3099129870 & -2.4530723295 & 4.4581327514 \\ \mathrm{H} & 0.9823252010 & -2.3484959653 & 1.5409606222 \\ \mathrm{H} & -0.3656013838 & -2.6625879489 & 2.6680939663 \\ \mathrm{H} & 2.4316511729 & -1.6186005326 & 3.3667300806 \\ \mathrm{H} & -1.1419660049 & -1.1711766381 & 4.5617837303 \\ \mathrm{H} & -2.3241166087 & 0.9244982606 & 4.0664586501 \\ \mathrm{H} & -0.7185559204 & 1.8603773984 & 4.0094762624 \\ & & & \end{array}$

$\mathrm{N}$

C

C

$\mathrm{N}$

$\mathrm{Ru}$

$\mathrm{Cl}$

$\mathrm{Cl}$
0.4360943295

$-0.0162436880$

$-1.4846691931$

$-1.5228008345$

$-0.4117956237$

$-2.8296963969$

0.0037658052

2.1949465781

1.7784768156

$-1.5773821086$

$-1.2081920014$

$-0.7618289382$

$-1.8901974108$

0.5675464676

0.1106634791

$-2.1636704770$

$-1.8036105609$

1.8619493715

2. 5241707346

1.9973733036
$-0.9565380443$

$-0.7693340385$

$-0.3799692332$

0.0139329647

$-0.4063461434$

0.3147767058

$-0.3303701163$

0.6907500274

$-1.4598044946$

1.3336992219

2. 4777307568

1. 6105756385

$-1.7356437875$

0.0288629011

$-1.6873263866$

$-1.2301407333$

0.4427198480

$-2.4766629267$

$-0.8181137052$

$-1.4704494410$

$$
\begin{array}{r}
-0.8951814772 \\
-2.2743277224 \\
-2.0771685645 \\
-0.6626928372 \\
0.0147289431 \\
-0.0981041024 \\
1.9218051798 \\
1.9659268123 \\
-0.6628926179 \\
3.4766990342 \\
3.8280279582 \\
2.1014955658 \\
2.5669954768 \\
-2.7563585400 \\
-2.8585226031 \\
-2.2411920010 \\
-2.7260714811 \\
-1.0670248675 \\
-1.1486201451 \\
0.4037350697
\end{array}
$$




$\begin{array}{rrrr}\mathrm{H} & -3.1492812186 & 1.3286471881 & -0.3735860102 \\ \mathrm{H} & -3.5704754526 & -0.3967649935 & -0.4864764194 \\ \mathrm{H} & -2.8025267853 & 0.1983968793 & 0.9818968416 \\ \mathrm{H} & -1.4438422117 & 2.0229476518 & 1.3578137039 \\ \mathrm{H} & -0.7532884451 & 3.4119684796 & 4.0804299598 \\ \mathrm{H} & -2.2059522279 & 0.4682554426 & 3.6455674748 \\ \mathrm{H} & 0.1680693074 & 2.1838342078 & 2.1607349861\end{array}$

25

$\begin{array}{lr}\mathrm{C} & -1.1707727049 \\ \mathrm{~N} & -1.2748282421 \\ \mathrm{C} & -0.1403282775 \\ \mathrm{~N} & 0.7946642132 \\ \mathrm{C} & 0.3425956294 \\ \mathrm{Ru} & 0.2058405480 \\ \mathrm{Cl} & -1.0704492196 \\ \mathrm{C} & 2.1868339833 \\ \mathrm{C} & -2.6051562497 \\ \mathrm{C} & -0.3326821445 \\ \mathrm{Cl} & 2.3399556524 \\ \mathrm{H} & 0.8077576065 \\ \mathrm{H} & 0.6142915127 \\ \mathrm{H} & -1.7138472895 \\ \mathrm{H} & -1.6025007264 \\ \mathrm{H} & 2.5063538668 \\ \mathrm{H} & 2.8352132969 \\ \mathrm{H} & 2.2898193844 \\ \mathrm{H} & -3.1364281416 \\ \mathrm{H} & -3.1703130200 \\ \mathrm{H} & -2.5288007181 \\ \mathrm{C} & -1.3000471988 \\ \mathrm{H} & -0.7877657170 \\ \mathrm{C} & -1.3335381190 \\ \mathrm{H} & -1.8364613130 \\ \mathrm{H} & -1.9021877411 \\ \mathrm{H} & 0.5357316280\end{array}$

$$
\begin{array}{r}
0.1707098755 \\
0.4782303519 \\
0.1509761444 \\
-0.2139726487 \\
0.0155225596 \\
0.1336846035 \\
-1.9065045263 \\
-0.5467592416 \\
0.6071764581 \\
2.2457606750 \\
1.0144100621 \\
0.9260776851 \\
-0.8277007980 \\
-0.7600482888 \\
0.9750077010 \\
-1.2953843485 \\
0.3342742782 \\
-0.9691225341 \\
1.4245180917 \\
-0.3241174480 \\
0.8182341258 \\
2.0424528969 \\
2.5199276345 \\
0.7222926516 \\
2.8141837596 \\
0.0323076488 \\
2.8691288208
\end{array}
$$

0.8634765695

0.5426489829

0.1643953982

0.3858215425

0.5367405828

0.5689013811

$-1.7978570385$

$-0.1633418194$

1.0552937046

2. 7539077394

2. 5151912808

1. 2126489419

1.1672557559

1. 2962969752

$-0.4594983791$

$-0.3975516455$

1. 3337937008
$-2.0843096273$

$-0.6495603166$

0.0064719961

$-0.8950707695$

$-2.2736717924$

1. 9666999445

1.8820321481

$-0.6322344430$

$-0.0728775845$

1. 9012638867

2.4322308150

$-2.6776967463$

$-2.9157726888$

$-2.3020127116$

$-2.6878068738$

$-1.3644674322$

$-0.7027211847$

0.3673703877

$-0.5737320856$

$-0.2059725254$

0.9911831225

3. 0194671658

0.9466056622

3.1648263796

3.5785449256

3. 7801364715

2.1127674080
$-1.0727574361$

$-0.5525751253$

$-1.5534482380$

$-2.8711392663$

$-2.5057805224$

1. 3829290825

1. 7961159250

$-1.4423328465$

$-0.3402752673$

1. 3494659830

2. 6809796774

2.7007185562

1. 7812200158

$-3.3318698826$

$-3.5367149367$

$-2.6497498737$

$-3.0611608462$ 


$\begin{array}{lr}\mathrm{H} & 2.5865792732 \\ \mathrm{H} & 2.9695518757 \\ \mathrm{H} & 2.5374918548 \\ \mathrm{H} & -2.8972516120 \\ \mathrm{H} & -2.9677634456 \\ \mathrm{H} & -2.1178534594 \\ \mathrm{H} & -1.0709735716 \\ \mathrm{H} & -0.1926430981 \\ \mathrm{H} & 1.1830520027 \\ \mathrm{H} & -0.4160091445\end{array}$

27

$\begin{array}{lr}\mathrm{N} & -1.2276199770 \\ \mathrm{C} & 0.0155032072 \\ \mathrm{~N} & 0.9056987865 \\ \mathrm{C} & 0.2687165859 \\ \mathrm{C} & -1.2167829991 \\ \mathrm{Ru} & 0.6281852620 \\ \mathrm{Cl} & -0.0390298775 \\ \mathrm{C} & 2.3533471234 \\ \mathrm{C} & -2.4612493286 \\ \mathrm{C} & 1.1065915691 \\ \mathrm{C} & 0.4416263306 \\ \mathrm{C} & -0.6009267517 \\ \mathrm{Cl} & 2.3681636802 \\ \mathrm{H} & 0.4773743868 \\ \mathrm{H} & 0.6538766311 \\ \mathrm{H} & -1.5772471220 \\ \mathrm{H} & -1.8698359084 \\ \mathrm{H} & 2.7654851287 \\ \mathrm{H} & 2.7826079537 \\ \mathrm{H} & 2.6328233391 \\ \mathrm{H} & -3.2815906821 \\ \mathrm{H} & -2.6736463739 \\ \mathrm{H} & -2.3616769524 \\ \mathrm{H} & -1.4091735693 \\ \mathrm{H} & 0.9416593268 \\ \mathrm{H} & 2.1184041799 \\ \mathrm{H} & 0.5630705026 \\ & \end{array}$

-0.8856627636
0.7268614734
-0.6195741882
1.8952853448
0.1537888139
1.2666480330
0.5635192184
3.1880280799
3.4164270834
2.9591672612
$-2.2265610350$

$-1.5566701582$

$-0.4722083007$

$-0.7716969003$

$-0.3957450505$

0.7057573432

3. 4572281964

3. 5440523406

1. 2855762863

0.5728835188

$$
\begin{array}{rr}
0.7445527795 & -1.0210814765 \\
0.7173668293 & -0.5041290924 \\
0.6365397980 & -1.5056329818 \\
0.6010061286 & -2.8295745137 \\
0.4456476321 & -2.4649749241 \\
0.6025351857 & 1.4661561136 \\
-1.6820755093 & 1.1667106854 \\
0.6357023875 & -1.3831119836 \\
0.5173743515 & -0.2846310129 \\
2.9286777647 & 1.2913940943 \\
2.5888181901 & 2.4668068752 \\
1.6062814469 & 2.3853442422 \\
-0.0704194598 & 2.9313249990 \\
1.5345762854 & -3.3699905758 \\
-0.2340352058 & -3.4235721407 \\
-0.5787922593 & -2.6286106614 \\
1.1346938794 & -3.0095581857 \\
-0.3184835435 & -1.7311153738 \\
1.4495470907 & -1.9817181364 \\
0.7779811199 & -0.3371524892 \\
1.0219748715 & -0.8055061905 \\
-0.5555994582 & -0.2047787536 \\
0.9296970793 & 0.7175492072 \\
1.3666316352 & 3.0750172297 \\
2.7426339226 & 3.4265677648 \\
3.3204091002 & 1.3513770688 \\
3.1209585319 & 0.3745604702
\end{array}
$$

28

$\begin{array}{lr}\mathrm{N} & 1.0606826318 \\ \mathrm{C} & 0.6344362188 \\ \mathrm{C} & -0.8701899459 \\ \mathrm{~N} & -1.1285117531 \\ \mathrm{C} & 0.0051567238 \\ \mathrm{C} & -2.4818369883 \\ \mathrm{Ru} & -0.0987193780 \\ \mathrm{C} 1 & 0.3792002690 \\ \mathrm{C} & 2.4303847709 \\ \mathrm{C} & 1.4704317553 \\ \mathrm{C} & 0.9529690268 \\ \mathrm{C} & 0.2992958801 \\ \mathrm{C} 1 & -1.5816953650 \\ \mathrm{H} & 0.8370255816\end{array}$

$$
\begin{array}{r}
-0.1873698677 \\
-0.1556087234 \\
-0.4416480024 \\
-0.1814480836 \\
-0.0810196022 \\
-0.2620284612 \\
0.3432352675 \\
2.5699287855 \\
0.1939793138 \\
-0.2662777977 \\
-1.5197171417 \\
-2.4132234450 \\
1.0033050715 \\
0.8388266141
\end{array}
$$$$
\text { (1) }
$$

$-1.1111974500$

$-2.5222930596$

$-2.4195822625$

$-0.9972933650$

$-0.2771613836$

$-0.4726863851$

1. 7254220268

1.0945853955

$-0.8029578472$

2. 4140693742

2. 9301790000

2.1358607268

3. 4491167601

$-2.9435170473$ 


$\begin{array}{lr}\mathrm{H} & 1.1781221064 \\ \mathrm{H} & -1.1172098254 \\ \mathrm{H} & -1.4779974358 \\ \mathrm{H} & 2.5937993600 \\ \mathrm{H} & 2.6256393733 \\ \mathrm{H} & 3.1121407523 \\ \mathrm{H} & -3.1157786614 \\ \mathrm{H} & -2.9065809377 \\ \mathrm{H} & -2.4814390909 \\ \mathrm{H} & 2.3388383346 \\ \mathrm{H} & 0.8883402500 \\ \mathrm{H} & -0.3024945377 \\ \mathrm{H} & 0.4308932725\end{array}$

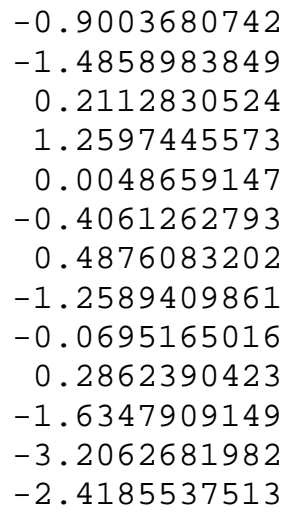

$-0.9003680742$

$-1.4858983849$

0.2112830524

0.0048659147

$-0.4061262793$

0.4876083202

$-1.2589409861$

$-0.0695165016$

0.2862390423

$-1.6347909149$

$-2.4185537513$
$-3.1115211773$

$-2.6599650914$

$-3.0536459661$

$-1.0051698648$

0.2510117694

$-1.4149188159$

$-0.9587706739$

$-0.6532458480$

0.6028271230

2. 7774729426

4.0155543690

2. 5711065915

1. 0598168765
29

$\begin{array}{lr}\mathrm{C} & -0.0865145494 \\ \mathrm{~N} & 1.0225606839 \\ \mathrm{C} & 0.7442187803 \\ \mathrm{C} & -0.7891355779 \\ \mathrm{~N} & -1.1428781470 \\ \mathrm{C} & 2.4123500483 \\ \mathrm{C} & -2.5370425841 \\ \mathrm{Ru} & -0.1631254689 \\ \mathrm{Cl} & -2.1419198153 \\ \mathrm{Cl} & 1.0004209511 \\ \mathrm{C} & 1.0143540286 \\ \mathrm{C} & 1.4076856685 \\ \mathrm{C} & 0.6415559277 \\ \mathrm{H} & 1.1559239939 \\ \mathrm{H} & 1.2106439107 \\ \mathrm{H} & -1.1710202396 \\ \mathrm{H} & -1.2349366224 \\ \mathrm{H} & 2.8990564031 \\ \mathrm{H} & 2.4708305926 \\ \mathrm{H} & 2.9377389641 \\ \mathrm{H} & -3.1506785359 \\ \mathrm{H} & -2.9014447261 \\ \mathrm{H} & -2.6437220488 \\ \mathrm{H} & 1.4293407952 \\ \mathrm{H} & 2.3537237387 \\ \mathrm{H} & 0.9593650827 \\ \mathrm{H} & -0.3370127777\end{array}$

-0.0955649180
-0.2030576790
-0.8023668392
-0.8714060545
-0.5454517265
-0.0896883660
-0.6533625609
0.5253429914
1.8412099938
-1.4046450441
1.9319724018
2.8667958261
3.1973940683
-0.1855165473
-1.7953981064
-1.8619674594
-0.1368178177
0.7477407232
0.0508095029
-1.0179292347
0.0925898653
-1.6558336702
-0.4900030761
2.1445858069
3.3909819492
3.9583613336
2.7501212099
0.0296404217

0.8012614561

2. 1122908422

2.1345554150

0.7491163110

0.3923723058

0.3541233955

$-1.8511145107$

$-1.8063649681$

$-2.5846473849$

$-1.7772399858$

$-0.7434610845$

0.3146370565

2.9188408396

2.1711097415

2.4059044754

2.8195293096

0.9065038227

$-0.6833710253$

0.6496758323

0.8740451126

0.6107036100

$-0.7151082547$

$-2.7791652821$

$-0.8979752827$

1.0223934487

0.4567072741

30

C $\quad-1.2426844806$

0.4577509873

$-2.4769241367$

0.5465837554

$-0.9952296817$

$-1.2138602133$

0.5006057410

$-0.5026531564$

0.0463159626

0.3330101553

0.9051394228
0.2066711340

0.0903986982

$-2.4511042353$

0.7969518539

0.3433563628

$-1.5408020278$

$-2.8214380741$

$-0.2990040733$

1.3993017747

2.7173843729

$-0.3998774240$

0.5019246891

$-1.9740307257$

$-1.5018933206$

1. 2450893651

1.4983918966 


\begin{tabular}{|c|c|c|c|}
\hline C & 0.5716592645 & 2.2769102705 & 2.6507188721 \\
\hline $\mathrm{C}$ & -0.3926922452 & 1.1953271915 & 2.4688935435 \\
\hline $\mathrm{H}$ & 0.6320126209 & 0.7117857092 & -3.6132532027 \\
\hline $\mathrm{H}$ & 0.3175740519 & -0.9618791972 & -3.1086092092 \\
\hline $\mathrm{H}$ & -1.9602493300 & -0.3005969086 & -2.7967891012 \\
\hline $\mathrm{H}$ & -1.5516389949 & 1.4226985370 & -2.8978887427 \\
\hline C & -1.5489475342 & 0.8990047569 & 3.2971935080 \\
\hline $\mathrm{C}$ & 1.0262679348 & 2.6212739030 & 4.0354010241 \\
\hline $\mathrm{C}$ & 2.4125223519 & 3.5909777255 & 1.4652380196 \\
\hline $\mathrm{H}$ & 0.5230266100 & 2.9273099437 & 0.6227459727 \\
\hline $\mathrm{C}$ & 2.4642317586 & 4.7859007189 & 0.7226451607 \\
\hline $\mathrm{C}$ & 3.6216198439 & 5.5663101125 & 0.6973970432 \\
\hline $\mathrm{C}$ & 4.7548420382 & 5.1542107140 & 1.4048925126 \\
\hline $\mathrm{C}$ & 4.7219721454 & 3.9557115854 & 2.1276414317 \\
\hline $\mathrm{C}$ & 3.5651198469 & 3.1772155405 & 2.1603767239 \\
\hline $\mathrm{H}$ & 1.5805197719 & 5.1134446691 & 0.1787981426 \\
\hline $\mathrm{H}$ & 3.6366675343 & 6.4944068064 & 0.1306462905 \\
\hline $\mathrm{H}$ & 5.6586948090 & 5.7585590665 & 1.3878861073 \\
\hline $\mathrm{H}$ & 5.6047657018 & 3.6220504557 & 2.6672079959 \\
\hline $\mathrm{H}$ & 3.5509740734 & 2.2342266768 & 2.6995898238 \\
\hline $\mathrm{C}$ & 1.1494444868 & 3.9790526166 & 4.3805967902 \\
\hline $\mathrm{C}$ & 1.4672560455 & 4.3558326470 & 5.6857777917 \\
\hline C & 1.6741222100 & 3.3794552211 & 6.6650576984 \\
\hline $\mathrm{C}$ & 1.5531456370 & 2.0274149540 & 6.3302443685 \\
\hline $\mathrm{C}$ & 1.2238783928 & 1.6470040527 & 5.0280977556 \\
\hline $\mathrm{H}$ & 0.9915120717 & 4.7400692087 & 3.6214574068 \\
\hline $\mathrm{H}$ & 1.5550240590 & 5.4104905175 & 5.9351845085 \\
\hline $\mathrm{H}$ & 1.9278972571 & 3.6707064349 & 7.6815610611 \\
\hline $\mathrm{H}$ & 1.7184823676 & 1.2616994727 & 7.0841315193 \\
\hline $\mathrm{H}$ & 1.1559983064 & 0.5965101625 & 4.7695023787 \\
\hline $\mathrm{C}$ & -2.1999560308 & 1.9547907037 & 3.9748387416 \\
\hline $\mathrm{C}$ & -3.2777380051 & 1.6976782560 & 4.8179598961 \\
\hline $\mathrm{C}$ & -3.7173682877 & 0.3822108062 & 5.0095207832 \\
\hline $\mathrm{C}$ & -3.0833580945 & -0.6704171914 & 4.3395210289 \\
\hline $\mathrm{C}$ & -2.0140601169 & -0.4201153923 & 3.4820921998 \\
\hline $\mathrm{H}$ & -1.8425445783 & 2.9719968237 & 3.8456534013 \\
\hline $\mathrm{H}$ & -3.7641779388 & 2.5187055187 & 5.3382999340 \\
\hline $\mathrm{H}$ & -4.5466725285 & 0.1788985684 & 5.6829392125 \\
\hline $\mathrm{H}$ & -3.4206176643 & -1.6927287992 & 4.4898603050 \\
\hline $\mathrm{H}$ & -1.5096725898 & -1.2303260420 & 2.9650283657 \\
\hline $\mathrm{C}$ & 3.1983169556 & -0.5788875303 & -1.2158832906 \\
\hline $\mathrm{C}$ & 4.5788170603 & -0.3359268233 & -1.1976292780 \\
\hline $\mathrm{C}$ & 5.1206876582 & 0.9208157292 & -1.4692536518 \\
\hline $\mathrm{C}$ & 4.2457513800 & 1.9603444826 & -1.8054032704 \\
\hline $\mathrm{C}$ & 2.8631558690 & 1.7728558702 & -1.8364339633 \\
\hline $\mathrm{C}$ & 2.7097691050 & -1.9783024707 & -0.9310965267 \\
\hline $\mathrm{H}$ & 5.2434302300 & -1.1631818951 & -0.9557970808 \\
\hline $\mathrm{C}$ & 6.6107403594 & 1.1546111122 & -1.3841740096 \\
\hline $\mathrm{H}$ & 4.6476792914 & 2.9451302587 & -2.0340449987 \\
\hline $\mathrm{C}$ & 1.9730202130 & 2.9203286590 & -2.2553549385 \\
\hline $\mathrm{H}$ & 1.0682244030 & 2.9849703339 & -1.6443628130 \\
\hline $\mathrm{H}$ & 2.5069367458 & 3.8698252067 & -2.1656678069 \\
\hline $\mathrm{H}$ & 1.6515416858 & 2.8213106629 & -3.3022994913 \\
\hline $\mathrm{H}$ & 6.8947711130 & 2.1069940059 & -1.8447690680 \\
\hline $\mathrm{H}$ & 6.9392753324 & 1.1826041955 & -0.3363835208 \\
\hline $\mathrm{H}$ & 7.1725529363 & 0.3527690152 & -1.8785532593 \\
\hline $\mathrm{H}$ & 3.3672844171 & -2.7067954682 & -1.4180257698 \\
\hline $\mathrm{H}$ & 2.7177332733 & -2.1796766096 & 0.1445722869 \\
\hline $\mathrm{H}$ & 1.6863069216 & -2.1439166181 & -1.270607526 \\
\hline
\end{tabular}




$\begin{array}{ll}\mathrm{C} & -2.8356064322 \\ \mathrm{C} & -4.0574439448 \\ \mathrm{C} & -4.9047815101 \\ \mathrm{C} & -4.5259828891 \\ \mathrm{C} & -3.3179111058 \\ \mathrm{C} & -1.9948206882 \\ \mathrm{H} & -4.3489047606 \\ \mathrm{C} & -6.1816694656 \\ \mathrm{H} & -5.1863595212 \\ \mathrm{C} & -2.9865707895 \\ \mathrm{H} & -1.9868922347 \\ \mathrm{H} & -3.7125148298 \\ \mathrm{H} & -3.0226275529 \\ \mathrm{H} & -2.6301846326 \\ \mathrm{H} & -1.4622381037 \\ \mathrm{H} & -1.2477112503 \\ \mathrm{H} & -6.9182307960 \\ \mathrm{H} & -5.9809301092 \\ \mathrm{H} & -6.6342297343\end{array}$
2. 1202139611
2. 3370031517
1.2835947516
$-0.0138730942$
$-0.2827163454$
3. 3235644172
3. 3588921770
1. 5354282406
$-0.8460912038$
$-1.6985587697$
$-1.9866059138$
$-2.3971991033$
$-1.8238924235$
4.1334794149
3. 7114895513
3. 0978581607
0.7444439511
1. 5626603986
2. 4946814232

-0.0105772351
0.6369417470
0.9846490941
0.6233037019
-0.0260410567
-0.3728463870
0.8737453222
1.7491473334
0.8573367837
-0.4304941430
-0.0912022942
-0.0022272271
-1.5216639186
-0.7479071844
0.5030367497
-1.1386766653
1.5723829087
2.8284325552
1.4742157745

31

$\begin{array}{lrrr}\mathrm{N} & 1.3450380081 & -0.1701986658 & -0.6431894286 \\ \mathrm{C} & 1.1975110623 & -0.8626858462 & -1.9480898286 \\ \mathrm{C} & -0.2647751533 & -1.3191848346 & -1.9422013543 \\ \mathrm{~N} & -0.7457090154 & -0.8388703415 & -0.6326808175 \\ \mathrm{C} & 0.2109528115 & -0.1865098612 & 0.0950361555 \\ \mathrm{C} & -2.1558068582 & -0.9595893371 & -0.3376775143 \\ \mathrm{Ru} & -0.3110890322 & 0.1838138462 & 2.0184287210 \\ \mathrm{C} & 0.8721409094 & 1.3154922469 & 2.9230306200 \\ \mathrm{C} & 0.5847096973 & 1.2610832593 & 4.3588701477 \\ \mathrm{C} & 1.2550974557 & 2.0519092202 & 5.2549079486 \\ \mathrm{C} & 2.5885352749 & 0.5370936614 & -0.4778601931 \\ \mathrm{Cl} & 0.7284606544 & -1.9421639725 & 2.5384937803 \\ \mathrm{Cl} & -1.7999481087 & 2.0500190071 & 1.4760699541 \\ \mathrm{H} & 1.4273017062 & -0.1678208286 & -2.7627056166 \\ \mathrm{H} & 1.9038579002 & -1.6970406321 & -2.0026690221 \\ \mathrm{H} & -0.3711400868 & -2.4070540435 & -2.0073450487 \\ \mathrm{H} & -0.8556739622 & -0.8678989545 & -2.7467479211 \\ \mathrm{C} & 1.8519621317 & 2.3210354191 & 2.4538124519 \\ \mathrm{C} & -0.4905267938 & 0.2925943234 & 4.7721898423 \\ \mathrm{C} & 1.1100781272 & 2.2056349775 & 6.7028593989 \\ \mathrm{H} & 2.0466867610 & 2.6695987519 & 4.8414488117 \\ \mathrm{C} & 3.2281237877 & 2.1409681660 & 2.6825333690 \\ \mathrm{C} & 4.1240300984 & 3.1837551167 & 2.4437996448 \\ \mathrm{C} & 3.6601245043 & 4.4181803689 & 1.9771404888 \\ \mathrm{C} & 2.2961128807 & 4.5941494463 & 1.7254936876 \\ \mathrm{C} & 1.3936001981 & 3.5529647177 & 1.9548012954 \\ \mathrm{H} & 3.5844099010 & 1.1889684561 & 3.0664013098 \\ \mathrm{H} & 5.1843330398 & 3.0333496095 & 2.6314487842 \\ \mathrm{H} & 4.3576317735 & 5.2351887293 & 1.8082665902 \\ \mathrm{H} & 1.9279657096 & 5.5497576153 & 1.3593957767 \\ \mathrm{H} & 0.3294999167 & 3.6870632836 & 1.7808792369 \\ \mathrm{C} & -0.1582086112 & -0.8735969180 & 5.4889923699 \\ \mathrm{C} & -1.1495382117 & -1.7562746532 & 5.9009282094 \\ \mathrm{C} & -2.4956195843 & -1.4988393709 & 5.5991530352 \\ \mathrm{C} & -2.8388372897 & -0.3499946102 & 4.8910347037 \\ \mathrm{C} & -1.8428303829 & 0.5523681395 & 4.4828566697\end{array}$




\begin{tabular}{|c|c|c|c|}
\hline $\mathrm{H}$ & 0.8844847120 & -1.0824832889 & 5.7045804573 \\
\hline & -0.8767414306 & -2.6542983902 & 6.4489830330 \\
\hline & -3.2671990100 & -2.1948252766 & 5.9193970495 \\
\hline $\mathrm{H}$ & -3.8783227268 & -0.1349655757 & 4.65803838 \\
\hline $\mathrm{H}$ & -2.1208386252 & 1.4659834517 & 3.96906002 \\
\hline & 2.1840039958 & 2.8194295701 & 7.38629213 \\
\hline C & 2.1485837535 & 3.0198925496 & 8.76385817 \\
\hline c & 1.0221910880 & 2.6310025081 & 9.49534671 \\
\hline C & -0.0628437828 & 2.0463342583 & 8.8329674 \\
\hline c & -0.0242607702 & 1.8317619256 & 7.45707657 \\
\hline $\mathrm{H}$ & 3.0571479705 & 3.1322185881 & 6.81806573 \\
\hline $\mathrm{H}$ & 2.9937106632 & 3.4847650976 & 9.26489774 \\
\hline $\mathrm{H}$ & 0.9850644471 & 2.7909080904 & 10.56990195 \\
\hline $\mathrm{H}$ & -0.9494074764 & 1.7580756812 & \\
\hline $\mathrm{H}$ & -0.8824831298 & 1.3938216396 & 6.9647498 \\
\hline c & 2.6605049031 & 1.8631318284 & -0.9532384 \\
\hline c & 3.9111371036 & 2.4797566368 & -1.01089510 \\
\hline c & 5.0791134573 & 1.8157128345 & -0.61553486 \\
\hline c & 4.9721189284 & 0.5042278473 & -0.1483378 \\
\hline c & 3.7405127604 & -0.1627220882 & -0.0799 \\
\hline c & 1.4251011357 & 2.5977987755 & -1.41975 \\
\hline $\mathrm{H}$ & 3.9739407032 & 3.5046209613 & -1.37072 \\
\hline C & 6.4137688692 & 2.5207369107 & -0.669679391 \\
\hline $\mathrm{H}$ & 5.8697467752 & -0.0266879318 & 0.16342746 \\
\hline C & 3.6865277553 & -1.6006044778 & 0.3776110 \\
\hline $\mathrm{H}$ & 3.5496319089 & -2.2859927225 & -0 . \\
\hline $\mathrm{H}$ & 4.6253898025 & -1.8799960884 & 0.866 \\
\hline $\mathrm{H}$ & 2.8619611732 & -1.7786356695 & 1.07450886 \\
\hline $\mathrm{H}$ & 7.2436839803 & 1.8220255892 & -0.521977534 \\
\hline $\mathrm{H}$ & 6.5618299025 & 3.0267463710 & -1.63125356 \\
\hline $\mathrm{H}$ & 6.4814699145 & 3.2893931918 & 0.111 \\
\hline $\mathrm{H}$ & 1.6652108270 & 3.6395161075 & -1 \\
\hline $\mathrm{H}$ & 1.002616 & 0198045 & -2 \\
\hline$\Pi$ & 0.6390797493 & 2.5946679174 & -0.65831702 \\
\hline c & -2.6520178041 & -2.1295320236 & 0.277337856 \\
\hline C & -4.0277918135 & -2.2094053258 & 0.527241681 \\
\hline C & -4.9117028090 & -1.1958295516 & 0.146644234 \\
\hline C & -4.3986571579 & -0.0960575960 & -0.547595012 \\
\hline C & -3.03 & 0.0379702428 & -0.812 \\
\hline C & -1.7754802829 & -3.3177986783 & 0.59302070 \\
\hline 11 & -4.4150919720 & -3.1005960558 & 1.016898525 \\
\hline $\mathrm{C}$ & -6.3834399073 & -1.2864902507 & 0.474739633 \\
\hline $\mathrm{H}$ & -5.0754082171 & 0.6851010710 & -0.887969635 \\
\hline C & -2.5582212343 & 1.2032448002 & -1.648 \\
\hline $\mathrm{H}$ & -1.5537854910 & 1.5252820696 & -1.374412683 \\
\hline $\mathrm{H}$ & -2.5602134988 & 0.9385885609 & -2.716848543 \\
\hline $\mathrm{H}$ & -3.2233156213 & 2.0615888538 & -1.52074372 \\
\hline $\mathrm{H}$ & -6.9948619332 & -0.7776381642 & -0.278542252 \\
\hline $\mathrm{H}$ & -6.7168879461 & -2.3279066039 & 0.541397851 \\
\hline $1+$ & -6.5980862344 & -0.8119751278 & 1.441785094 \\
\hline $\mathrm{H}$ & -2.3785395479 & -4.1239644464 & 1.022875626 \\
\hline $\mathrm{H}$ & -1.3056599289 & -3.7115930017 & -0.31850964 \\
\hline & -0.9711180266 & -3.0793101164 & 1.292491801 \\
\hline
\end{tabular}


6-Me $\mathrm{M}_{1}$ 4-isomer

$\begin{array}{lrrr}\text { C1 } & -0.153337 & 0.592564 & -2.175150 \\ \text { C2 } & -1.659785 & 0.470652 & -1.921929 \\ \text { N3 } & -1.714617 & 0.524578 & -0.453764 \\ \text { C4 } & -0.516736 & 0.245540 & 0.098734 \\ \text { N5 } & 0.407730 & 0.176392 & -0.877925 \\ \text { C6 } & -2.993785 & 0.403160 & 0.229010 \\ \text { Ru7 } & -0.270956 & 0.036982 & 2.113717 \\ \text { C18 } & 0.576931 & 2.330023 & 2.169246 \\ \text { C9 } & 1.847949 & 0.232877 & -0.683967 \\ \text { C10 } & 1.356986 & -0.695070 & 2.551366 \\ \text { C11 } & 0.156997 & -0.235989 & 4.468633 \\ \text { C12 } & -0.954501 & 0.261092 & 4.226591 \\ \text { C13 } & -2.285519 & 0.850942 & 4.446259 \\ \text { C114 } & -1.371520 & -2.144931 & 2.013984 \\ \text { H15 } & 0.144034 & 1.628257 & -2.394361 \\ \text { H16 } & 0.204814 & -0.054070 & -2.981563 \\ \text { H17 } & -2.061989 & -0.489201 & -2.277712 \\ \text { H18 } & -2.241946 & 1.282718 & -2.367745 \\ \text { H19 } & 2.185386 & 1.260497 & -0.501937 \\ \text { H20 } & 2.122642 & -0.383989 & 0.171217 \\ \text { H21 } & 2.336386 & -0.166454 & -1.578135 \\ \text { H22 } & -3.713854 & 1.073583 & -0.251541 \\ \text { H23 } & -3.365879 & -0.627399 & 0.209224 \\ \text { H24 } & -2.888859 & 0.711924 & 1.271219 \\ \text { H25 } & 2.238363 & -0.067746 & 2.726981 \\ \text { H26 } & 1.480175 & -1.779042 & 2.654232 \\ \text { H27 } & 0.986220 & -0.624295 & 5.020937 \\ \text { H28 } & -2.494166 & 0.921261 & 5.520244 \\ \text { H29 } & -2.328635 & 1.857301 & 4.015611 \\ \text { H30 } & -3.058250 & 0.228611 & 3.982009 \\ & & & \end{array}$

\section{6-Me ${ }_{1}$ 3-isomer}

$\begin{array}{lrrr}\text { N1 } & -2.296842 & 0.847954 & 0.043375 \\ \text { C2 } & -1.029862 & 0.498942 & 0.319079 \\ \text { N3 } & -0.303750 & 0.534778 & -0.813717 \\ \text { C4 } & -1.060631 & 1.158860 & -1.912731 \\ \text { C5 } & -2.503927 & 1.103777 & -1.389685 \\ \text { Ru6 } & -0.383769 & 0.153392 & 2.231699 \\ \text { C17 } & -1.464782 & -2.043695 & 2.227470 \\ \text { C8 } & 1.148698 & 0.499236 & -0.886644 \\ \text { C9 } & -3.426820 & 0.759635 & 0.954307 \\ \text { C10 } & -0.718077 & 0.292849 & 4.329132 \\ \text { C11 } & 0.423321 & -0.194745 & 4.507021 \\ \text { C12 } & 1.584859 & -0.679815 & 5.276580 \\ \text { C113 } & 0.522847 & 2.428282 & 2.183983 \\ \text { C14 } & 1.284076 & -0.601684 & 2.458506 \\ \text { H15 } & 1.407115 & -1.687665 & 2.517080 \\ \text { H16 } & -1.633239 & 0.681538 & 4.737252 \\ \text { H17 } & -0.716109 & 2.192273 & -2.058957 \\ \text { H18 } & -0.918622 & 0.607650 & -2.847415 \\ \text { H19 } & -3.080716 & 0.279155 & -1.831478 \\ \text { H20 } & -3.052757 & 2.037265 & -1.549094 \\ \text { H21 } & 1.582704 & 1.481345 & -0.663494 \\ \text { H22 } & 1.527871 & -0.225272 & -0.165925 \\ \text { H23 } & 1.439659 & 0.181539 & -1.893011\end{array}$




$\begin{array}{lrrr}\text { H2 } & -3.758251 & 1.756845 & 1.268600 \\ \text { H25 } & -4.258635 & 0.249147 & 0.454686 \\ \text { H26 } & -3.148656 & 0.158696 & 1.823033 \\ \text { H27 } & 2.186052 & 0.018139 & 2.483791 \\ \text { H28 } & 2.481727 & -0.086286 & 5.069868 \\ \text { H29 } & 1.350667 & -0.586516 & 6.343537 \\ \text { H30 } & 1.804118 & -1.731431 & 5.063907\end{array}$

6-Me 2, 4-isomer

$\begin{array}{lrrr}\mathrm{N} & -0.1485374234 & 0.4754793343 & -0.7923267659 \\ \mathrm{C} & -0.8806790640 & 1.0292751331 & -1.9440317856 \\ \mathrm{C} & -2.3346759374 & 0.9326873782 & -1.4701428828 \\ \mathrm{~N} & -2.1628566441 & 0.8410567229 & -0.0134683107 \\ \mathrm{C} & -0.9075997016 & 0.4824924966 & 0.3225238734 \\ \mathrm{C} & -3.3311491785 & 0.7239890277 & 0.8439194665 \\ \mathrm{Ru} & -0.3463722993 & 0.1190651772 & 2.2577512930 \\ \mathrm{C} & 0.4352929453 & -0.2697655441 & 4.4517954531 \\ \mathrm{C} & -0.7555172790 & 0.0985973849 & 4.4182709658 \\ \mathrm{C} & -2.0896955272 & 0.4688237158 & 4.9204646221 \\ \mathrm{C} & 1.3048295944 & 0.4814221507 & -0.8206979880 \\ \mathrm{Cl} & 0.5874153179 & 2.3751192387 & 2.3251427157 \\ \mathrm{C} & 1.3438748455 & -0.6428615739 & 2.4279618072 \\ \mathrm{C} & 1.7340088775 & -2.0879190883 & 2.4107461271 \\ \mathrm{Cl} & -1.6527822501 & -1.9829617110 & 2.1645669458 \\ \mathrm{H} & -0.5724473542 & 2.0709306056 & -2.1157282953 \\ \mathrm{H} & -0.6800576702 & 0.4503282894 & -2.8505010253 \\ \mathrm{H} & -2.8340815388 & 0.0281331298 & -1.8470677468 \\ \mathrm{H} & -2.9377995545 & 1.8048057414 & -1.7405701733 \\ \mathrm{H} & 1.7037276200 & 1.4881220090 & -0.6445380272 \\ \mathrm{H} & 1.6844317190 & -0.1855489558 & -0.0481203757 \\ \mathrm{H} & 1.6382935203 & 0.1163133396 & -1.7974353056 \\ \mathrm{H} & -4.0418329302 & 1.5158125873 & 0.5829966524 \\ \mathrm{H} & -3.8077560289 & -0.2575564300 & 0.7419798626 \\ \mathrm{H} & -3.0410868555 & 0.8544860126 & 1.8875099920 \\ \mathrm{H} & 2.1807865367 & 0.0661458710 & 2.5011317440 \\ \mathrm{H} & 1.3723650993 & -0.5278552688 & 4.9006333857 \\ \mathrm{H} & -2.1072528517 & 0.4279826008 & 6.0160084631 \\ \mathrm{H} & -2.3501424044 & 1.4866514365 & 4.6087625300 \\ \mathrm{H} & -2.8382605606 & -0.2273346166 & 4.5273847064 \\ \mathrm{H} & 2.4734322969 & -2.3025798221 & 3.1970163581 \\ \mathrm{H} & 0.8757587332 & -2.7545264224 & 2.4958217531 \\ \mathrm{H} & 2.2386854099 & -2.2886230437 & 1.4524099679 \\ & & & \end{array}$

\section{6-Me 2 2, 3-isomer}

$\begin{array}{ll}\mathrm{N} & -0.2986402949 \\ \mathrm{C} & -1.0824929289 \\ \mathrm{C} & -2.5084281306 \\ \mathrm{~N} & -2.2493572868 \\ \mathrm{C} & -0.9917425988 \\ \mathrm{C} & -3.3544882777 \\ \mathrm{Ru} & -0.3614423111 \\ \mathrm{C} & 0.4036316348 \\ \mathrm{C} & 1.6202239801 \\ \mathrm{C} & 1.1464054467 \\ \mathrm{C} 1 & 0.6453244962\end{array}$

0.5582459596

1.1255694912

1.0708953403

0.9936118028

0.5881936018

0.9169214703

0.1616755257

$-0.3233576682$

$-0.3813578851$

0.4813507181

2. 3747670143
$-0.8154991787$

$-1.9256044658$

$-1.3639248506$

0.0807398756

0.3386011081

1. 0239819477

2.2423534082

4.4490846916

5.2881741798

$-0.9344323119$

2. 3953770874 


$\begin{array}{lrrr}\mathrm{C} & 1.3018080498 & -0.6618275515 & 2.4671338536 \\ \mathrm{C} & 1.6554348146 & -2.1141759873 & 2.3791014078 \\ \mathrm{Cl} & -1.6600904385 & -1.9415198723 & 1.9699646276 \\ \mathrm{C} & -0.8246814052 & -0.0921382378 & 4.2893333872 \\ \mathrm{H} & -1.8092488013 & 0.0106988686 & 4.7090449885 \\ \mathrm{H} & -0.7562657449 & 2.1571058956 & -2.1233756654 \\ \mathrm{H} & -0.9525728279 & 0.5353300146 & -2.8375692671 \\ \mathrm{H} & -3.0497178263 & 0.1737035884 & -1.6975347419 \\ \mathrm{H} & -3.1053705157 & 1.9543774165 & -1.6091724067 \\ \mathrm{H} & 1.5970431742 & 1.4822930809 & -0.9522627060 \\ \mathrm{H} & 1.5458166473 & -0.0665106645 & -0.0825101006 \\ \mathrm{H} & 1.4046513315 & -0.0500735938 & -1.8567479027 \\ \mathrm{H} & -4.0900568622 & 1.6867039027 & 0.7684077141 \\ \mathrm{H} & -3.8222102755 & -0.0738536596 & 1.0157197077 \\ \mathrm{H} & -2.9899936953 & 1.1205454463 & 2.0339804001 \\ \mathrm{H} & 2.1528447714 & 0.0259053267 & 2.5592663512 \\ \mathrm{H} & 2.4598753843 & -2.3738816393 & 3.0820070378 \\ \mathrm{H} & 0.7908315060 & -2.7634429765 & 2.5172681363 \\ \mathrm{H} & 2.0555101997 & -2.2980928671 & 1.3695769467 \\ \mathrm{H} & 2.2567531406 & 0.4887010034 & 5.0923114228 \\ \mathrm{H} & 1.3210862312 & -0.3564959095 & 6.3418446161 \\ \mathrm{H} & 2.2081153603 & -1.2887914232 & 5.1179558564\end{array}$




\section{First-Generation Phosphane Complexes}

\section{$1\left(1^{\text {st }}\right)$}

$\begin{array}{ll}\text { C } & -4.3984808855 \\ \text { P } & -2.8031773110 \\ \text { C } & -2.2316294051 \\ \text { Ru } & -2.8961123155 \\ \text { Cl } & -4.9518446744 \\ \text { P } & -2.6135640676 \\ \text { C } & -1.8460823049 \\ \text { C } & -3.7488376737 \\ \mathrm{Cl} & -0.6301114972 \\ \mathrm{C} & -4.0917247051 \\ \mathrm{C} & -1.4362544802 \\ \mathrm{H} & -4.8447474608 \\ \mathrm{H} & -3.1903122248 \\ \mathrm{H} & -3.8103362778 \\ \mathrm{H} & -4.7850643254 \\ \mathrm{H} & -4.6020528027 \\ \mathrm{H} & -1.2534777692 \\ \mathrm{H} & -1.8455008491 \\ \mathrm{H} & -0.4931906795 \\ \mathrm{H} & -1.5773437135 \\ \mathrm{H} & -0.9570305971 \\ \mathrm{H} & -2.5518261474 \\ \mathrm{H} & -4.7336976936 \\ \mathrm{H} & -5.1418629915 \\ \mathrm{H} & -4.2937816861 \\ \mathrm{H} & -1.2352420440 \\ \mathrm{H} & -2.9285159585 \\ \mathrm{H} & -2.1888518298 \\ \mathrm{C} & -1.6444884096 \\ \mathrm{H} & -2.0013013133 \\ \mathrm{H} & -0.6638022416 \\ \mathrm{H} & -1.5698765392 \\ & \end{array}$

$2\left(1^{\text {st }}\right)$

$\begin{array}{ll}\mathrm{C} & -4.3609083044 \\ \mathrm{P} & -2.8011564883 \\ \mathrm{C} & -2.0898093314 \\ \mathrm{C} & -1.6768247234 \\ \mathrm{Ru} & -2.9259183314 \\ \mathrm{Cl} & -0.6433800923 \\ \mathrm{Cl} & -4.8474706066 \\ \mathrm{P} & -2.7783623696 \\ \mathrm{C} & -2.1953241341 \\ \mathrm{C} & -3.8217709748 \\ \mathrm{C} & -1.6098673252 \\ \mathrm{C} & -4.3601951191 \\ \mathrm{H} & -4.8057720431 \\ \mathrm{H} & -3.4117618152 \\ \mathrm{H} & -4.2333351517 \\ \mathrm{H} & -4.7121974146 \\ \mathrm{H} & -5.1045280097 \\ \mathrm{H} & -2.1407104740 \\ \mathrm{H} & -2.8915166639\end{array}$

1.2925042541

1.0552609762

$-0.6084840033$

1.0503679597

$-0.2812970104$

0.3695401933

1.6013113310

2.6305627236

1.9969847109

$-0.2381162610$

$-1.0508127998$

2.6944406283

3.5629387629

$-0.6646135322$

0.5938731540

$-0.9847581097$

$-1.3880128806$

$-1.8839772798$

$-0.7315130728$

1.1460482820

2.0080555820

2. 4196056532

2. 3258761927

0.6270300431

1.0835367697

$-0.8098259484$

$-1.3630154660$

$-0.6654053170$

2. 2284874302

3.2515296195

2.1428443456

2. 0282129165
1.3430938347

2.2279101426

1.6700090542

4.6298501753

4.4508861816

6.9184062779

8.0485731224

4.8928773810

4.7486303973

7.8289039847

6.9543969363

4.9037645208

5.0480061791

8.7983976021

7.9855695034

7.2154000699

7.9810537384

6.3739209443

6.5008697569

9.0083910593

7.5593149224

8.2231144869

1.4771558014

1.7899652162

0.2725322459

2.0763386118

2.0471216877

0.5763410654

1.4136771412

1.5675076018

1.8881363121

0.3388482654
0.7270657409

1. 0478021389

2.5388449771

$-0.3164413112$

1.1051956069

1.9894634982

$-0.4130784065$

0.6777820292

$-1.0491632060$

2. 6809757997

1.7107603951

0.7655083552

2.7543890431

3. 6192451788

0.4122215527

1.8020987076

0.1559944943

$-1.2844556555$

$-1.7349480952$
1. 3034536508

2. 2270543334

1. 4152268146

1.7026231240

4.6310482685

4.5940220683

4.6173477223

6.9964628566

7.2758479946

4.7591570714

7.9741598807

7.9346965923

5.2422573668

4.3610669313

8.9641784052

7.9541783600

7.4152399979

8.3448278455

6.7840520525 


$\begin{array}{ll}\mathrm{H} & -1.2025117202 \\ \mathrm{H} & -1.5140051893 \\ \mathrm{H} & -0.6366515257 \\ \mathrm{H} & -1.9710871215 \\ \mathrm{H} & -5.0352125049 \\ \mathrm{H} & -4.8495430071 \\ \mathrm{H} & -4.1691784101 \\ \mathrm{H} & -0.6931229525 \\ \mathrm{H} & -2.0715099452 \\ \mathrm{H} & -1.5804979631 \\ \mathrm{H} & -2.7787129140 \\ \mathrm{H} & -1.1475869755 \\ \mathrm{H} & -1.9212020709\end{array}$

-1.1746181719
1.3427647082
1.7075340448
2.7438009199
1.5801093034
-0.1509972588
0.5716959189
-0.1489232413
-1.2721022353
-0.3572101652
3.3810153446
2.7862494637
2.3680467991

6.8318484038

9.0018843255

7.4764264694

7.9921428788

1. 4289786166

1.7337572294

0.2357801973

2.1510966884

2.0623019208

0.6116350044

1. 5378137011

1. 9114819356

0.3460733761

\section{$3\left(1^{s t}\right)$}

$\begin{array}{ll}\mathrm{C} & -4.3618262145 \\ \mathrm{P} & -2.7599381302 \\ \mathrm{C} & -1.7607518034 \\ \mathrm{C} & -1.9654255569 \\ \mathrm{Ru} & -2.8914507616 \\ \mathrm{Cl} & -0.5151266408 \\ \mathrm{Cl} & -4.5976767962 \\ \mathrm{P} & -2.9110279350 \\ \mathrm{C} & -2.1789947484 \\ \mathrm{C} & -3.7037570382 \\ \mathrm{C} & -1.9425668788 \\ \mathrm{C} & -4.5655254500 \\ \mathrm{H} & -3.9947662123 \\ \mathrm{H} & -3.9477018680 \\ \mathrm{H} & -4.4887136744 \\ \mathrm{H} & -5.0449775113 \\ \mathrm{H} & -5.1891803008 \\ \mathrm{H} & -2.1853872015 \\ \mathrm{H} & -2.7538027687 \\ \mathrm{H} & -1.1489641177 \\ \mathrm{H} & -1.9304787497 \\ \mathrm{H} & -0.9207519852 \\ \mathrm{H} & -2.3775653633 \\ \mathrm{H} & -4.8677968971 \\ \mathrm{H} & -4.9982520655 \\ \mathrm{H} & -4.2169979830 \\ \mathrm{H} & -0.9596367203 \\ \mathrm{H} & -2.5517476741 \\ \mathrm{H} & -1.9016862737 \\ \mathrm{H} & -2.2228027609 \\ \mathrm{H} & -0.7641942252 \\ \mathrm{H} & -1.6808569310\end{array}$

1.1310600306

1.0594387893

2. 4129872919

$-0.4758967688$

1.0239255443

1.3469542667

$-0.6570781679$

0.7618965167

$-0.8559349459$

2. 6602343158

1. 9923671852

0.7563683046

3. 1446218798

3. 2520994784

0.5416768826

1. 7316988963

0.0015022001

$-0.9881755924$

$-1.6606394362$

$-0.9039699325$

1. 7455502930

2.0148783681

2. 9888129648

2.0770090723

0.3154958383

1.0477017456

$-0.5551178602$

$-1.3392456034$

$-0.4741297926$

3. 3800772869

2. 4001464245

2. 2996890326
1. 3074185586

2. 2190503071

1. 4636105160

1.5783463070

4. 6227411928

4.7158385852

4.4679528016

7.0160512036

7. 5117478439

4.6979221125

7.9904613468

7.8319133240

5.6384456618

3.8068327913

8.9037430144

7.6980064385

7.3449855281

8.5996450281

7.0432639830

7. 1453716107

9.0580230458

7.6015970186

7.8602272265

1.5270992282

1. 6618888718

0.2244471872

2. 0019529878

1. 9075678614

0.4842756172

1. 6878708747

1. 9136229209

0.3766529071

\section{$4\left(1^{\text {st }}\right)$}

$\begin{array}{lr}\text { C } & 0.7405966775 \\ \text { P } & -0.0057720653 \\ C & 0.7405966775 \\ \text { C } & -1.7642819791 \\ \text { Ru } & 0.1556486954 \\ \text { Cl } & -0.5725407134\end{array}$

2. 3450174107

1. 5126627245

2. 3450174107

2. 0449149864

$-0.7211996964$

$-0.9257776895$
1. 4585217902

0.0000000000

$-1.4585217902$

0.0000000000

0.0000000000

$-2.2321938508$ 


$\begin{array}{lr}\mathrm{Cl} & -0.5725407134 \\ \mathrm{C} & 1.9684212231 \\ \mathrm{H} & 2.4161898811 \\ \mathrm{H} & 2.6807030859 \\ \mathrm{H} & 1.8150174598 \\ \mathrm{H} & 0.2864408732 \\ \mathrm{H} & 0.5797446854 \\ \mathrm{H} & -2.2578938731 \\ \mathrm{H} & -2.2578938731 \\ \mathrm{H} & -1.8399258661 \\ \mathrm{H} & 1.8150174598 \\ \mathrm{H} & 0.2864408732 \\ \mathrm{H} & 0.5797446854\end{array}$

\section{$5\left(1^{\text {st }}\right)$}

$\begin{array}{lr}\mathrm{C} & -0.8835316709 \\ \mathrm{P} & 0.2842479113 \\ \mathrm{C} & 1.8901571034 \\ \mathrm{Ru} & -0.3489545829 \\ \mathrm{C} & -0.4823210882 \\ \mathrm{C} & -1.6399041480 \\ \mathrm{Cl} & -2.5776485911 \\ \mathrm{Cl} & 2.0033067660 \\ \mathrm{C} & 0.1483808692 \\ \mathrm{H} & 1.1681903976 \\ \mathrm{H} & -0.5778421148 \\ \mathrm{H} & -2.6975342784 \\ \mathrm{H} & 0.3781393773 \\ \mathrm{H} & -1.8548358296 \\ \mathrm{H} & -0.4791366770 \\ \mathrm{H} & -1.0323355406 \\ \mathrm{H} & 2.6606162297 \\ \mathrm{H} & 1.8007829476 \\ \mathrm{H} & 2.1587018049 \\ \mathrm{C} & 0.4658840587 \\ \mathrm{H} & 1.2313577559 \\ \mathrm{H} & 0.7525906056 \\ \mathrm{H} & -0.4839229979\end{array}$

\section{$5 b\left(1^{\text {st }}\right)$}

$\begin{array}{lr}\mathrm{C} & -0.7806277108 \\ \mathrm{P} & 0.3199763651 \\ \mathrm{C} & 0.3576477528 \\ \mathrm{C} & 1.9917636029 \\ \mathrm{Ru} & -0.3150615613 \\ \mathrm{C} & -0.1982340810 \\ \mathrm{C} & -0.3862492715 \\ \mathrm{C} & -1.5559095926 \\ \mathrm{Cl} & -2.5252336280 \\ \mathrm{Cl} & 1.8681661865 \\ \mathrm{H} & -0.4305310957 \\ \mathrm{H} & 0.0875835301 \\ \mathrm{H} & -2.6070496382 \\ \mathrm{H} & 0.6012059360 \\ \mathrm{H} & -1.7974492700\end{array}$
2.2321938508
0.0000000000
0.0000000000
0.0000000000
1. 4911103073
2. 3649525905
1. 4102835606
$-0.8895833254$
0.8895833254
0.0000000000
$-1.4911103073$
$-2.3649525905$
$-1.4102835606$

-1.0725444166
0.2432781590
-0.6390082543
1.6578342222
3.6775889472
3.2262761974
0.5952878979
2.4365477838
0.4277426000
0.4182525953
-0.2793093481
3.0935984790
4.3048318183
-0.6338356183
-1.5945972670
-1.7820145681
0.0692460278
-1.4239232593
-1.0955253302
1.3170260214
2.0702966993
0.7225389066
1.8200949537

-1.3688731377
0.0874179399
0.9067911670
-0.6646572969
1.5755075518
0.2812710241
3.2960183898
3.0098580681
1.4702977568
2.5613410892
0.5474245430
-0.7650584700
2.8529522162
3.6364168397
-1.0179990239 


$\begin{array}{rrrr}\mathrm{H} & -0.4316366063 & 0.7595968625 & -1.9813872538 \\ \mathrm{H} & -0.7911992182 & -0.9860425209 & -1.9818562708 \\ \mathrm{H} & 2.7347076858 & -0.5727581639 & 0.1322696573 \\ \mathrm{H} & 2.0353797072 & -1.4085199643 & -1.2661104577 \\ \mathrm{H} & 2.2173007074 & 0.3641937923 & -1.3061095850 \\ \mathrm{H} & 1.0701340505 & 1.2892797029 & 1.7360699503 \\ \mathrm{H} & 0.6526832775 & 2.1090549804 & 0.2022658202 \\ \mathrm{H} & -0.6374969876 & 1.5424084281 & 1.3042267469\end{array}$

\section{$5 c\left(1^{s t}\right)$}

\section{$6\left(1^{\text {st }}\right)$}

$\begin{array}{lr}\mathrm{C} & -1.5925207164 \\ \mathrm{P} & -0.1182289972 \\ \mathrm{Ru} & -0.4539701973 \\ \mathrm{Cl} & 1.4170897318 \\ \mathrm{C} & 0.5896772519 \\ \mathrm{C} & 1.0907412576 \\ \mathrm{C} & 0.5053495246 \\ \mathrm{Cl} & -2.4375258295 \\ \mathrm{C} & -1.2668868828 \\ \mathrm{C} & -0.5729590743 \\ \mathrm{H} & 1.2614069337 \\ \mathrm{H} & 1.5459341041 \\ \mathrm{H} & 0.0618551766 \\ \mathrm{H} & -1.9550006889 \\ \mathrm{H} & -0.1074689216 \\ \mathrm{H} & -1.3036321270 \\ \mathrm{H} & -2.0773079353 \\ \mathrm{H} & -2.3095244519 \\ \mathrm{H} & 2.0357873751 \\ \mathrm{H} & 0.7002142298\end{array}$

$$
\begin{array}{r}
2.6169316561000 \\
1.8053796583000 \\
-0.4953761450000 \\
-0.5040440484000 \\
2.6169316561000 \\
2.4471458389000 \\
-0.5001703182000 \\
-0.5001703182000 \\
-2.8878564524000 \\
-3.1238018716000 \\
-1.4437579102000 \\
0.3879856825000 \\
2.3712781191000 \\
2.2335868901000 \\
3.7055391623000 \\
2.0775981165000 \\
2.0775981165000 \\
3.5430529588000 \\
2.3712781191000 \\
2.2335868901000 \\
3.7055391623000 \\
-3.4236475558000 \\
-2.7513545980000
\end{array}
$$
1.4531664766000
0.0000000000000
0.0000000000000
0.0000000000000
$-1.4531664766000$
0.0000000000000
$-2.2629353289000$
2.2629353289000
0.0000000000000
0.0000000000000
0.0000000000000
0.0000000000000
1.4862430481000
2.3630462910000
1.4009124729000
$-0.8901102369000$
0.8901102369000
0.0000000000000
$-1.4862430481000$
$-2.3630462910000$
$-1.4009124729000$
0.0000000000000
0.0000000000000

0.8058698621

0.0848072240

$-1.9623572102$

$-1.3771788531$

1. 4885617285

$-0.1941898819$

$-3.3149760128$

$-2.4274863979$

$-3.2472744584$

$-4.1264914539$

0.7328738810

$-3.5164401599$

$-3.9280420853$

$-2.8691276814$

$-5.0735122793$

1. 6587488564

0.0322447700

1. 1398533874

$-0.5428606042$

$-0.9590690968$
-0.5920157689
0.2363979635
1.4484264575
2.9001073217
1.1901261141
-1.1254892350
0.6386698990
0.1073349042
2.9903240069
2.4461306656
-1.6845102765
0.9144258552
-0.1530548534
3.7227508694
2.2653279747
-1.2166102601
-1.1913660205
0.1642770818
-0.7003899671
-1.8023852758 


$\begin{array}{rrrr}\mathrm{H} & -0.1241305297 & 1.8103938648 & 1.9547173658 \\ \mathrm{H} & 1.4952280614 & 1.1487656785 & 1.6971998819 \\ \mathrm{H} & 0.8084061562 & 2.3318455707 & 0.5251670431\end{array}$

\section{$7\left(1^{\text {st }}\right)$}

$\begin{array}{ll}\mathrm{C} & -3.8001474034 \\ \mathrm{P} & -2.4772978399 \\ \mathrm{Ru} & -3.0948069587 \\ \mathrm{C} & -3.5567793916 \\ \mathrm{C} & -1.0682587965 \\ \mathrm{C} & -1.9242599546 \\ \mathrm{C} I & -0.8763920463 \\ \mathrm{Cl} & -5.0792019563 \\ \mathrm{C} & -3.9584517952 \\ \mathrm{H} & -3.6632542885 \\ \mathrm{H} & -4.1350572601 \\ \mathrm{H} & -4.6490953591 \\ \mathrm{H} & -3.4367320223 \\ \mathrm{H} & -1.0742717465 \\ \mathrm{H} & -2.7424831074 \\ \mathrm{H} & -1.6279676471 \\ \mathrm{H} & -1.3417009265 \\ \mathrm{H} & -0.2183949092 \\ \mathrm{H} & -0.7949038518 \\ \mathrm{C} & -3.3721381955 \\ \mathrm{H} & -4.7991488776 \\ \mathrm{H} & -3.7394144430 \\ \mathrm{H} & -2.4932712944 \\ & \end{array}$

1.5630896900

1.0720134893

1.0108594610

2. 7820410393

2.1922169299

$-0.5826538863$

0.8555548935

$-0.1882015618$

3.2661563689

3. 4758366313

2. 5810082230

0.8869177672

1. 5217672518

$-0.9071298781$

$-1.2970936770$

$-0.5490860637$

3. 2228232643

1. 9067820811

2. 1331593008

2. 9036319189

3. 9644218700

3. 2750265710

2. 2640654644
1.1482598118

2. 3267272919

4. 4789116034

4. 6200477545

1. 9508202439

1. 7455627837

5.2861945286

4. 0139647414

5.9324709129

3.7774264349

1. 3695446366

1.2767584310

0.1152495119

2. 3530756211

1.8740365045

0.6908577790

2. 1961902326

2.5759096308

0.8911896862

7.0925727177

5.9550692332

8.0450944430

7.1070835095

\section{$8\left(1^{s t}\right)$}

$\begin{array}{ll}\mathrm{C} & -3.8774344908 \\ \mathrm{P} & -2.5195553016 \\ \mathrm{C} & -1.7837033237 \\ \mathrm{Ru} & -3.1523298313 \\ \mathrm{Cl} & -4.9951358716 \\ \mathrm{C} & -1.2403009878 \\ \mathrm{C} & -3.7750412457 \\ \mathrm{C} & -4.1815248092 \\ \mathrm{C} & -5.4542756354 \\ \mathrm{C} 1 & -0.9425202487 \\ \mathrm{H} & -3.8807356413 \\ \mathrm{H} & -4.3243902193 \\ \mathrm{H} & -4.6480432087 \\ \mathrm{H} & -3.5017237700 \\ \mathrm{H} & -0.9113647922 \\ \mathrm{H} & -2.5216511472 \\ \mathrm{H} & -1.4809394417 \\ \mathrm{H} & -1.6316047937 \\ \mathrm{H} & -0.3735674683 \\ \mathrm{H} & -0.9460246056 \\ \mathrm{H} & -3.3685843249 \\ \mathrm{H} & -5.6945106451 \\ \mathrm{H} & -6.2883202413\end{array}$

1. 4288038148

1.0836813375

$-0.5001775343$

0.9540147676

$-0.4501278910$

2. 3535852570

2. 6620102316

3. 0884956702

3. 1881376752

1.0276883113

3. 3724997473

2. 4029893725

0.6630996459

1. 4305438321

$-0.7299175915$

$-1.2984738900$

$-0.4352585996$

3. 3479041122

2. 1672760871

2. 3222900304

3. 2929995322

3. 4858845416

2. 9612695088
1.1356657021

2. 3259913807

1. 7539279089

4. 4708129450

3. 9935574248

1. 9650092916

4. 6041757547

5.9558415256

6.3679167973

5. 3029828319

3. 7741719230

1. 3559058302

1. 2548618974

0.1062371130

2. 3727308167

1. 8750750330

0.7026713078

2. 2004422213

2. 6042171071

0.9098304384

6.6561357570

7.3848876615

5.7086729911 


\section{$9\left(1^{\text {st }}\right)$}

$\begin{array}{ll}\mathrm{C} & -3.8443917250 \\ \mathrm{P} & -2.4971427426 \\ \mathrm{C} & -1.9419994262 \\ \mathrm{Ru} & -3.0764256740 \\ \mathrm{Cl} & -5.0775501482 \\ \mathrm{C} & -1.1031386405 \\ \mathrm{C} & -3.5380031839 \\ \mathrm{C} & -4.0080509283 \\ \mathrm{C} & -4.3595032534 \\ \mathrm{Cl} & -0.8506566033 \\ \mathrm{H} & -3.4862530890 \\ \mathrm{H} & -4.1847985584 \\ \mathrm{H} & -4.6854600046 \\ \mathrm{H} & -3.4980786461 \\ \mathrm{H} & -1.0830970964 \\ \mathrm{H} & -2.7548473348 \\ \mathrm{H} & -1.6593633578 \\ \mathrm{H} & -1.3798823846 \\ \mathrm{H} & -0.2419825030 \\ \mathrm{H} & -0.8454160344 \\ \mathrm{H} & -4.0777671599 \\ \mathrm{H} & -4.7088954471 \\ \mathrm{H} & -4.3034763662\end{array}$

1. 6154157435

1.1091801563

$-0.5284915707$

0.9881639920

$-0.1892956558$

2. 2486808082

2. 7656090024

3. 2806063576

4. 5703553266

0.8030113648

3. 4985424468

2. 6241222608

0.9290435827

1. 6022065596

$-0.8610244916$

$-1.2502399118$

$-0.4693819744$

3. 2724523105

1. 9569208587

2. 2134882984

2. 5882412651

4. 9382052761

5. 2909825491
1.1870645861

2. 3311478611

1.7085783786

4.4877309133

4.0566863096

1.9583756369

4.6420325363

5. 9088179196

6.0896429018

5.2544905419

3.8264383146

1. 4394719082

1. 3133454243

0.1474109521

2. 2986953742

1.8304635051

0.6512502370

2. 2273652322

2. 5648018125

0.8937458975

6.7484194541

7.0498925930

5.2768015616

\section{$10\left(1^{\text {st }}\right)$}

$\begin{array}{lrrr}\mathrm{C} & 1.5369398749 & -0.0923009974 & 3.3267817379 \\ \mathrm{P} & -0.0044338914 & -0.4244226557 & 2.3778704492 \\ \mathrm{Ru} & 0.0664049234 & -0.0872801016 & -0.0002789371 \\ \mathrm{C} & 0.4259485580 & 1.7214959041 & 0.0000653381 \\ \mathrm{C} & -0.5338052647 & 2.8125065810 & 0.0014194453 \\ \mathrm{C} & -0.1330925388 & 4.1006331986 & -0.0019021716 \\ \mathrm{C} & -1.3144985349 & 0.4747622016 & 3.3083029717 \\ \mathrm{C} & -0.3659888086 & -2.2034853880 & 2.7131296403 \\ \mathrm{Cl} & -2.4088136753 & -0.1189494403 & 0.0055057525 \\ \mathrm{Cl} & 2.4490071938 & -0.6804681947 & -0.0066666503 \\ \mathrm{P} & -0.0005299905 & -0.4213040128 & -2.3787911469 \\ \mathrm{C} & 1.5247126878 & -0.0365651248 & -3.3338824754 \\ \mathrm{C} & -0.2981655383 & -2.2110822900 & -2.7117231523 \\ \mathrm{C} & -1.3453952315 & 0.4287286007 & -3.3047498399 \\ \mathrm{H} & 1.4833218213 & 2.0298524671 & -0.0014107786 \\ \mathrm{H} & 1.4464372732 & -0.3888714181 & -4.3686168025 \\ \mathrm{H} & 1.6839139554 & 1.0466063299 & -3.3335165307 \\ \mathrm{H} & 2.3764790918 & -0.5044625021 & -2.8335939600 \\ \mathrm{H} & -0.3435697362 & -2.4141768606 & -3.7877313699 \\ \mathrm{H} & 0.5125516062 & -2.7982709822 & -2.2692572224 \\ \mathrm{H} & -1.2440175810 & -2.5069426529 & -2.2477689104 \\ \mathrm{H} & -1.3932608603 & 0.0854273223 & -4.3442672336 \\ \mathrm{H} & -2.2931262671 & 0.2332983277 & -2.7960810622 \\ \mathrm{H} & -1.1651267752 & 1.5084553194 & -3.2903387257 \\ \mathrm{H} & 1.7362609938 & 0.9845461395 & 3.3208152540 \\ \mathrm{H} & 2.3706210841 & -0.5923614182 & 2.8260342275 \\ \mathrm{H} & 1.4493986553 & -0.4372323218 & 4.3633764838 \\ \mathrm{H} & -1.3232448952 & -2.4644599403 & 2.2502834522 \\ \mathrm{H} & 0.4231068202 & -2.8204971785 & 2.2701380642 \\ \mathrm{H} & -0.4167489061 & -2.4037877845 & 3.7896536140\end{array}$




$\begin{array}{lr}\mathrm{H} & -1.0937868304 \\ \mathrm{H} & -2.2708481774 \\ \mathrm{H} & -1.3707615341 \\ \mathrm{H} & -1.5906098185 \\ \mathrm{H} & -0.8452793048 \\ \mathrm{H} & 0.9225550325\end{array}$

\section{$11\left(1^{\text {st }}\right)$}

$\begin{array}{ll}\mathrm{C} & -4.0588391378 \\ \mathrm{P} & -2.5943122558 \\ \mathrm{Ru} & -2.8965308487 \\ \mathrm{C} & -3.5837730930 \\ \mathrm{C} & -1.2015250628 \\ \mathrm{C} & -2.2350999847 \\ \mathrm{Cl} & -0.5413350291 \\ \mathrm{Cl} & -4.9508244967 \\ \mathrm{C} & -3.2710407919 \\ \mathrm{C} & -2.8090823847 \\ \mathrm{C} & -3.1213182231 \\ \mathrm{H} & -4.4525596754 \\ \mathrm{H} & -4.2923041566 \\ \mathrm{H} & -4.9095485496 \\ \mathrm{H} & -3.8756949454 \\ \mathrm{H} & -1.3097596774 \\ \mathrm{H} & -3.0574083384 \\ \mathrm{H} & -2.1204985916 \\ \mathrm{H} & -1.4104544378 \\ \mathrm{H} & -0.2936457033 \\ \mathrm{H} & -1.0571780769 \\ \mathrm{H} & -1.7449741576 \\ \mathrm{H} & -2.5908467941 \\ \mathrm{H} & -4.3374001477 \\ \mathrm{H} & -3.4950850477 \\ \mathrm{C} & -3.7821137640 \\ \mathrm{H} & -2.1938871604 \\ \mathrm{H} & -3.4236412863 \\ \mathrm{H} & -4.7028313867\end{array}$

1. 5472817018

0.3133086491

0.1336060764

2. 5580907513

4. 9221669966

4.3659278646
3.2927316263
2. 8035031812
4.3483158331
0.0051156676
$-0.0010145333$
$-0.0057519946$

$\begin{array}{rr}1.7358306583 & 1.3125396477 \\ 1.1453420865 & 2.2560391076 \\ 0.8458303090 & 4.5616818899 \\ 2.5406299719 & 4.8756427767 \\ 2.1787258923 & 1.6329223851 \\ -0.5159385563 & 1.5405684441 \\ 1.2661611097 & 4.9455076391 \\ -0.3508182332 & 4.0817396679 \\ 0.6645441583 & 7.0292734778 \\ -0.5240725489 & 6.5615972936 \\ 3.8168205408 & 4.3760971068 \\ 2.6103000685 & 5.5445362323 \\ 2.7628914870 & 1.6100389091 \\ 1.0991644530 & 1.5693008844 \\ 1.7055028843 & 0.2327642801 \\ -0.9057442792 & 1.9767843079 \\ -1.1930200356 & 1.7879357238 \\ -0.4566462673 & 0.4523017291 \\ 3.2394808353 & 1.7991310484 \\ 1.9165545180 & 2.1817913566 \\ 2.0150566086 & 0.5591843923 \\ -0.7438574737 & 6.5716199339 \\ 1.4186175929 & 7.4130767288 \\ 0.8444787436 & 7.1290053396 \\ -1.3188033213 & 6.2843715476 \\ 4.9653400395 & 4.6342839934 \\ 3.8422532058 & 3.8106553948 \\ 5.9260460909 & 4.2744344802 \\ 4.9708392812 & 5.2138116741\end{array}$

1.7358306583

1. 3125396477

2.2560391076

4.5616818899

1. 6329223851

1. 5405684441

4. 9455076391

4.0817396679

7.0292734778

4. 3760971068

5.5445362323

1. 6100389091

1.5693008844

1.9767843079

1.7879357238

0.4523017291

1.7991310484

2.1817913566

0.5591843923

6.5716199339

7.4130767288

7.1290053396

6.2843715476

3. 8106553948

5.2138116741

\section{$12\left(1^{\text {st }}\right)$}

$\begin{array}{lr}\mathrm{C} & -1.4400890805 \\ \mathrm{P} & 0.0745703140 \\ \mathrm{C} & 0.3284893506 \\ \mathrm{C} & 1.4504055406 \\ \mathrm{Ru} & -0.2497599076 \\ \mathrm{C} 1 & -1.9916463617 \\ \mathrm{C} & 0.9402381723 \\ \mathrm{C} & -0.4352281550 \\ \mathrm{C} & -1.3291237445 \\ \mathrm{C} 1 & 1.2383535388 \\ \mathrm{C} & 0.9415345579 \\ \mathrm{C} & 2.0614615789 \\ \mathrm{H} & -1.3254594568 \\ \mathrm{H} & 1.8592215036 \\ \mathrm{H} & -2.3667920725 \\ \mathrm{H} & -1.1195030911\end{array}$

-0.6177973477
-0.0330811661
1.6846072076
-1.0058147548
-0.1360395153
1.5718428983
0.8408932448
-0.0245563582
-0.9412636411
-2.0622272636
2.2856006373
2.9260946043
-0.5260675519
0.3250737561
-0.6376879635
-2.0051240831
0.7639559602

$-0.1048507503$

0.5004711786

0.6264281571

$-2.4828986394$

$-2.1968616711$

$-3.5856785832$

$-4.7685174901$

$-4.1092628587$

$-2.6313390280$

$-3.8020659420$

$-4.1803292392$

1.8498733324

$-3.8844352292$

$-3.9931645466$

$-4.1808534351$ 


$\begin{array}{lr}\mathrm{H} & 0.3515661537 \\ \mathrm{H} & -0.8668920262 \\ \mathrm{H} & 0.0248038635 \\ \mathrm{H} & 2.0907067010 \\ \mathrm{H} & 2.9859952881 \\ \mathrm{H} & 1.3407400881 \\ \mathrm{H} & 2.4004101673 \\ \mathrm{H} & 1.4605309729 \\ \mathrm{H} & -1.6279335929 \\ \mathrm{H} & -2.2873830454 \\ \mathrm{H} & -0.4949855623 \\ \mathrm{H} & 0.3677275476 \\ \mathrm{H} & 1.2672865702\end{array}$

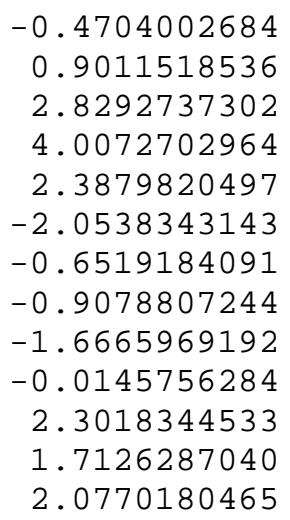

$-5.3720096082$

$-5.1369719572$

$-3.5922735973$

$-4.2859649539$

$-4.3807590738$

0.3384664519

0.2165025804

1. 7178996781

0.5130857905

0.4265769079

0.1325477080

1. 5952821856

0.0974092612

\section{$13\left(1^{\text {st }}\right)$}

\begin{tabular}{|c|c|}
\hline $\mathrm{C}$ & -0.7184308668 \\
\hline $\mathrm{P}$ & 0.1432903698 \\
\hline $\mathrm{C}$ & 1.5903142996 \\
\hline $\mathrm{H}$ & 2.2009387412 \\
\hline $\mathrm{Ru}$ & -1.0644177569 \\
\hline $\mathrm{Cl}$ & -2.9950868585 \\
\hline $\mathrm{C}$ & -1.3822613443 \\
\hline $\mathrm{C}$ & -2.1515876738 \\
\hline $\mathrm{C}$ & -2.3829935274 \\
\hline $\mathrm{C}$ & -1.9413393940 \\
\hline $\mathrm{C}$ & -2.6081010902 \\
\hline $\mathrm{Cl}$ & 0.9837876967 \\
\hline $\mathrm{H}$ & -0.5043322405 \\
\hline $\mathrm{H}$ & -2.0355663584 \\
\hline $\mathrm{H}$ & -3.1650889659 \\
\hline $\mathrm{H}$ & -3.3741720589 \\
\hline $\mathrm{H}$ & -1.6263062183 \\
\hline $\mathrm{H}$ & -1.0221797332 \\
\hline $\mathrm{H}$ & -2.2586155295 \\
\hline $\mathrm{H}$ & -3.5203672753 \\
\hline $\mathrm{H}$ & -1.5664465613 \\
\hline $\mathrm{H}$ & -1.1167800884 \\
\hline $\mathrm{H}$ & -0.0288657855 \\
\hline $\mathrm{H}$ & 1.2419505042 \\
\hline $\mathrm{H}$ & 2.1805455124 \\
\hline $\mathrm{C}$ & 0.8456067779 \\
\hline $\mathrm{H}$ & 1.4418631646 \\
\hline $\mathrm{H}$ & 0.0329274794 \\
\hline $\mathrm{H}$ & 1.464129170 \\
\hline
\end{tabular}

-0.9241232091
0.0523731741
0.8120519461
0.0423313068
1.7172876390
1.2890020590
3.6519965928
3.3558036929
1.8272824721
1.3833236038
0.4503188265
2.1637540601
4.2814047937
3.9319968366
3.7308374176
1.4787137236
3.8463417193
1.8143556887
0.1119404337
-0.0045163920
-1.4495819014
-0.2477225628
-1.6438849429
1.5216528371
1.3499011756
-1.1898917317
-0.6625307110
-1.7205040179
-1.9162310268

$-0.9817967800$

0.3110235868

$-0.5306667675$

$-1.0161925605$

1.5063669933

0.0693856758

1.2646817750

2. 6143217404

3. 0131387840

4.3401555267

5.0394991769

2. 7682914808

1.4077706798

0.4399772178

2.4544156314

2.7215204185

3.4333340708

4.7285367757

6.0107460671

4.6585726936

$-0.5357515857$

$-1.7411941947$

$-1.4377938518$

$-1.2871065976$

0.2155395317

1.4705340384

2.2194273431

1. 9766271170

0.9313183178

\section{$14\left(1^{\text {st }}\right)$}

$\begin{array}{lrrr}\mathrm{C} & 1.0268190092 & 0.0702924859 & -1.0589111997 \\ \mathrm{P} & -0.1285348871 & -0.2670561484 & 0.3323560484 \\ \mathrm{C} & -0.4466644711 & -2.0786662533 & 0.2237650877 \\ \mathrm{Ru} & 0.7790932767 & 0.2819656861 & 2.4796694243 \\ \mathrm{Cl} & -1.2586243022 & 1.5280179953 & 3.0130870163 \\ \mathrm{C} & -1.7126923083 & 0.5094467359 & -0.1777657135 \\ \mathrm{C} & 1.7869644529 & 1.8376140666 & 2.5365949602 \\ \mathrm{C} & 1.9269328227 & 0.8459890290 & 4.4197053142 \\ \mathrm{C} & 0.8633518661 & -0.0833401757 & 4.6330185402\end{array}$




$\begin{array}{lrrr}\mathrm{C} & 1.0415014112 & -1.5369687040 & 4.7002627732 \\ \mathrm{C} & 0.0360341725 & -2.3570799549 & 5.0583124081 \\ \mathrm{Cl} & 2.7503116461 & -1.0441842184 & 1.8401345505 \\ \mathrm{H} & 0.6417836306 & -0.3571039697 & -1.9931623398 \\ \mathrm{H} & 2.8441800562 & 1.8429485859 & 2.2547447986 \\ \mathrm{H} & 1.3328789719 & 2.8032971304 & 2.7739068307 \\ \mathrm{H} & 1.8013971374 & 1.8319423215 & 4.8669852758 \\ \mathrm{H} & -0.0610542425 & 0.3106657856 & 5.0519791931 \\ \mathrm{H} & 2.9446439197 & 0.4767965113 & 4.3115829923 \\ \mathrm{H} & 2.0125622457 & -1.9374988793 & 4.4185019936 \\ \mathrm{H} & 0.1711040187 & -3.4337961582 & 5.1015147682 \\ \mathrm{H} & -0.9461999690 & -1.9698286361 & 5.3204457734 \\ \mathrm{H} & 1.1448596906 & 1.1503312191 & -1.1784582246 \\ \mathrm{H} & 1.9967133097 & -0.3664538990 & -0.8131495699 \\ \mathrm{H} & -2.4989749488 & 0.2192698875 & 0.5223181724 \\ \mathrm{H} & -1.6171993351 & 1.5970965460 & -0.1303709354 \\ \mathrm{H} & -1.9838838772 & 0.1995805556 & -1.1938104167 \\ \mathrm{H} & -1.1818579488 & -2.3712397660 & 0.9783808135 \\ \mathrm{H} & -0.8269434796 & -2.3504363331 & -0.7680318942 \\ \mathrm{H} & 0.4905381134 & -2.6043011574 & 0.4206902590\end{array}$

\section{$15\left(1^{\text {st }}\right)$}

$\begin{array}{lrrr}\mathrm{C} & -4.3393233886 & 0.9521912877 & 1.3529168050 \\ \mathrm{P} & -2.7470942006 & 1.0167740794 & 2.2728746122 \\ \mathrm{Ru} & -2.8806851755 & 1.0358431877 & 4.5985504233 \\ \mathrm{C} & -3.8456016105 & 2.5882980074 & 4.5605797201 \\ \mathrm{C} & -1.8592969365 & 2.4344105006 & 1.5047960492 \\ \mathrm{C} & -1.8273064349 & -0.4685580828 & 1.6949801559 \\ \mathrm{Cl} & -0.5644115635 & 1.6835355644 & 4.6397102594 \\ \mathrm{Cl} & -4.5702733606 & -0.6749364216 & 4.4128947508 \\ \mathrm{C} & -2.7758154440 & 0.0624323562 & 6.9435408444 \\ \mathrm{C} & -2.7657694638 & 1.4187344993 & 7.0805404779 \\ \mathrm{H} & -4.1648798179 & 3.0789321790 & 5.4858926884 \\ \mathrm{H} & -4.1704178547 & 3.1076004311 & 3.6504928036 \\ \mathrm{H} & -4.9257784506 & 1.8520926625 & 1.5621561037 \\ \mathrm{H} & -4.9096560131 & 0.0858561552 & 1.6950294594 \\ \mathrm{H} & -4.1623156760 & 0.8864380929 & 0.2739284803 \\ \mathrm{H} & -0.8250744278 & -0.4549299801 & 2.1330758873 \\ \mathrm{H} & -2.3520903977 & -1.3647289143 & 2.0385959018 \\ \mathrm{H} & -1.7495629700 & -0.4857858824 & 0.6019575618 \\ \mathrm{H} & -2.3984343125 & 3.3635931046 & 1.7153333746 \\ \mathrm{H} & -0.8636087825 & 2.5088997635 & 1.9480653115 \\ \mathrm{H} & -1.7828863668 & 2.3046403845 & 0.4195730915 \\ \mathrm{H} & -3.6878743818 & 1.9490178440 & 7.2925314258 \\ \mathrm{H} & -3.7258259040 & -0.4644394159 & 6.9822787118 \\ \mathrm{C} & -1.5796169126 & -0.7708537815 & 6.8230713699 \\ \mathrm{H} & -1.8398138184 & 1.9818372496 & 7.1369506402 \\ \mathrm{C} & -1.6393570103 & -2.1064176356 & 6.6926637355 \\ \mathrm{H} & -0.6196508938 & -0.2605623992 & 6.8376738922 \\ \mathrm{H} & -0.7396663847 & -2.7102623876 & 6.6185571363 \\ \mathrm{H} & -2.5914159173 & -2.6295986953 & 6.6476532230\end{array}$

\section{$16\left(1^{\text {st }}\right)$}

$\begin{array}{llll}\mathrm{C} & -4.2779830884 & 1.3238834176 & 1.3253934314 \\ \mathrm{P} & -2.7242043268 & 1.0392964132 & 2.2689111777\end{array}$




$\begin{array}{ll}\mathrm{C} & -1.5419326141 \\ \mathrm{C} & -2.1096869664 \\ \mathrm{Ru} & -2.9259737567 \\ \mathrm{Cl} & -0.5452524821 \\ \mathrm{Cl} & -4.8599863147 \\ \mathrm{C} & -3.5486435917 \\ \mathrm{H} & -3.8161019535 \\ \mathrm{H} & -3.6908089760 \\ \mathrm{H} & -4.6736052388 \\ \mathrm{H} & -5.0167973367 \\ \mathrm{H} & -4.1026056078 \\ \mathrm{H} & -1.1361591909 \\ \mathrm{H} & -2.8130579323 \\ \mathrm{H} & -2.0101809383 \\ \mathrm{H} & -1.8823028058 \\ \mathrm{H} & -0.5669696991 \\ \mathrm{H} & -1.4572962020 \\ \mathrm{C} & -3.2200634236 \\ \mathrm{C} & -2.7887080534 \\ \mathrm{H} & -4.2805407822 \\ \mathrm{H} & -3.4919894348 \\ \mathrm{H} & -1.7303377240 \\ \mathrm{H} & -2.5189962654\end{array}$

2.2684890773

$-0.5843396328$

0.9966055864

1. 1493106359

$-0.3960236400$

2. 7190429811

3.2362078895

3. 3270173025

2. 3185612776

0.5820031027

1. 2494421823

$-0.7879893831$

$-1.3648640499$

$-0.5868243635$

3. 2816196404

2.1177558136

2.1648899165

1. 2537628744

$-0.0130329546$

1. 4797387707

$-0.8321174634$

$-0.2545030970$

2. 0578383914
1.5782181180

1.6613475125

4.5842588770

4.7727866824

4.3597994482

4.5811807560

5.5079588211

3. 6794453187

1. 5537407640

1. 6387670921

0.2465726204

2.1169154636

1. 9659539740

0.5700911280

1. 8142873643

2. 0482690238

0.4908406741

7.0742394354

6.8689839969

7.1363603171

6.7539775182

6.8971429613

7.2778123409

\section{$16 \mathrm{~b}\left(1^{\text {st }}\right)$}

$\begin{array}{ll}\mathrm{C} & -4.2246414770 \\ \mathrm{P} & -2.7461215771 \\ \mathrm{Ru} & -3.0811589881 \\ \mathrm{C} & -3.5573132049 \\ \mathrm{C} & -1.4601379984 \\ \mathrm{C} & -2.2141728058 \\ \mathrm{Cl} & -0.6841840950 \\ \mathrm{Cl} & -5.0304727563 \\ \mathrm{C} & -4.0503336197 \\ \mathrm{C} & -2.7563451804 \\ \mathrm{H} & -3.8326554813 \\ \mathrm{H} & -3.6027879315 \\ \mathrm{H} & -4.5608274460 \\ \mathrm{H} & -5.0310587137 \\ \mathrm{H} & -3.9904336585 \\ \mathrm{H} & -1.2788632970 \\ \mathrm{H} & -2.9814560961 \\ \mathrm{H} & -2.0649802951 \\ \mathrm{H} & -1.7414918421 \\ \mathrm{H} & -0.5112736543 \\ \mathrm{H} & -1.3500944593 \\ \mathrm{H} & -2.4644683394 \\ \mathrm{H} & -4.8631971857 \\ \mathrm{H} & -4.3442671106 \\ \mathrm{H} & -1.9731171066 \\ \mathrm{H} & \end{array}$

1.4457231835

1.0360581440

1.0539428970

2. 8130162872

2.1942034623

$-0.6146595106$

0.9051267984

$-0.2774362540$

0.8399044579

1. 2136752005

3. 3262026193

3. 4471605101

2. 4622225397

0.7528893382

1. 3795678340

$-0.8892078735$

$-1.3499227825$

$-0.6063907994$

3. 2245433922

1. 9737568037

2. 0988507614

2. 2517561127

1. 5585136754

$-0.2042777828$

0.4809301043
1. 2368737455

2. 2558083939

4. 5892134872

4.5575765751

1. 6261266266

1. 6468806472

4.7415829897

4.1228812750

6.8222389220

7.0081761733

5.4875736117

3. 6645961885

1. 4646045697

1. 4876313860

0.1686381262

2.1429544820

1.9056139033

0.5613614885

1. 8657646397

2.1201642645

0.5402059099

7.1326633827

6.7886191580

6.8311849671

7.1704624919

\section{$16 \mathrm{c}\left(1^{\mathrm{st}}\right)$}

$\begin{array}{lrrr}\mathrm{C} & -0.8486809727 & 0.2973887093 & -1.1316299447 \\ \mathrm{P} & 0.2961492273 & -0.2018274570 & 0.2151181934 \\ \mathrm{C} & 0.4636630420 & 1.3060649164 & 1.2590806164\end{array}$




$\begin{array}{lr}\mathrm{C} & 1.9179961329 \\ \mathrm{Ru} & -0.3519254705 \\ \mathrm{C} & 0.0914201731 \\ \mathrm{C} & -0.4181405546 \\ \mathrm{C} & -1.7009571369 \\ \mathrm{C} I & -2.6112263397 \\ \mathrm{Cl} & 1.9812560661 \\ \mathrm{H} & 1.1086035763 \\ \mathrm{H} & -0.6497671941 \\ \mathrm{H} & -2.4060092067 \\ \mathrm{H} & -0.0643915994 \\ \mathrm{H} & -1.8290190873 \\ \mathrm{H} & -0.4408189690 \\ \mathrm{H} & -0.9793202335 \\ \mathrm{H} & 2.6727941825 \\ \mathrm{H} & 1.8451011278 \\ \mathrm{H} & 2.1965597296 \\ \mathrm{H} & 1.2170511624 \\ \mathrm{H} & 0.7625178916 \\ \mathrm{H} & -0.4938083018 \\ \mathrm{H} & 0.2394192732 \\ \mathrm{H} & -2.1240135552\end{array}$

\section{$17\left(1^{\text {st }}\right)$}

$\begin{array}{lrr}\mathrm{P} & -0.0446871623 & -0.1712902086 \\ \mathrm{C} & 0.9992406161 & 0.6929371323 \\ \mathrm{H} & 0.6679348461 & 0.4527968261 \\ \mathrm{Ru} & 0.6362180334 & 0.3136460012 \\ \mathrm{C} & 2.0479534735 & 1.4626092860 \\ \mathrm{C} & 1.5968178249 & 1.0135570075 \\ \mathrm{C} & 0.5174469388 & 0.1553100545 \\ \mathrm{H} & 0.6930863426 & -0.9046804679 \\ \mathrm{Cl} & 2.1046864311 & -1.6358304494 \\ \mathrm{Cl} & -0.9536510187 & 2.1614579452 \\ \mathrm{H} & 3.0760439227 & 1.0924970214 \\ \mathrm{H} & 1.8999297959 & 2.5480611892 \\ \mathrm{H} & 1.4510723024 & 2.0842932186 \\ \mathrm{H} & -0.4682835641 & 0.5617208389 \\ \mathrm{H} & 2.6083205779 & 0.6234721304 \\ \mathrm{H} & 0.9293210558 & 1.7727966289 \\ \mathrm{H} & 2.0402072010 & 0.3820031183 \\ \mathrm{C} & -1.7626715498 & 0.3261458588 \\ \mathrm{H} & -2.4717528704 & -0.2476904912 \\ \mathrm{H} & -1.8984436212 & 1.3826337145 \\ \mathrm{H} & -1.9640433714 & 0.1429770259 \\ \mathrm{C} & 0.0483320611 & -1.9423340765 \\ \mathrm{H} & -0.6519382731 & -2.5260728109 \\ \mathrm{H} & -0.2030879969 & -2.0648647825 \\ \mathrm{H} & 1.0547352506 & -2.3150758511\end{array}$

-0.6328122697
1.6544164236
0.4538827589
3.7123500599
3.2267721881
0.6981076261
2.4652972461
0.4134692782
-0.2492759420
3.5212618658
4.4085057932
-0.7134882517
-1.6637049482
-1.8290023631
0.1001096825
-1.3977181327
-1.1108865543
2.0284978583
0.6501375014
1.7409051447
3.5716049227
2.6755122944
0.1333271125

$-1.1130816337$

$-2.1296532250$

2.3780498619

2.1668006195

4. 4986021704

4.6266373089

4.7886076812

2.3925085995

2. 5060423183

2.0803868714

2.1327668726

4.6013322710

4.8353342968

4.5518483973

$-0.9546304767$

$-0.9879124397$

$-0.2984548322$

0.3062400256

$-0.0560720187$

$-1.3600025837$

$-0.3556906973$

0.2497418496

$-1.4153108734$

$-0.1518608844$

\section{$18\left(1^{\text {st }}\right)$}

$\begin{array}{lr}\mathrm{C} & -0.7287268647 \\ \mathrm{P} & 0.1751912846 \\ \mathrm{C} & 0.8954395471 \\ \mathrm{C} & 1.5998055088\end{array}$
$-0.9388844493$
0.0602844257
$-1.1510121669$
0.7955073609

-0.9333603067
0.3119354532
1.4898411640
-0.5847943949 


$\begin{array}{lr}\text { Ru } & -1.0222452793 \\ \mathrm{Cl} & 1.0616083466 \\ \mathrm{Cl} & -3.0601480907 \\ \mathrm{C} & -1.3880270802 \\ \mathrm{C} & -2.1011142443 \\ \mathrm{C} & -2.0388954975 \\ \mathrm{H} & -1.4461715661 \\ \mathrm{H} & 2.1938759996 \\ \mathrm{H} & -0.5213015781 \\ \mathrm{H} & -2.0873278572 \\ \mathrm{H} & -3.1577892826 \\ \mathrm{H} & -3.0077981800 \\ \mathrm{H} & -1.6023021472 \\ \mathrm{H} & -1.5673092367 \\ \mathrm{H} & -1.1448141653 \\ \mathrm{H} & -0.0591723028 \\ \mathrm{H} & 1.2347926510 \\ \mathrm{H} & 2.2102701450 \\ \mathrm{H} & 1.5036317196 \\ \mathrm{H} & 0.0916536856 \\ \mathrm{H} & 1.5062260251 \\ & \end{array}$

\section{$19\left(1^{\text {st }}\right)$}

$\begin{array}{lr}\mathrm{C} & -0.8555198997 \\ \mathrm{P} & 0.3075838335 \\ \mathrm{C} & 0.6031369046 \\ \mathrm{C} & 1.8783005027 \\ \mathrm{Ru} & -0.3807525994 \\ \mathrm{C} & 0.1104782591 \\ \mathrm{C} & -0.4749078938 \\ \mathrm{C} & -1.6393207518 \\ \mathrm{Cl} & -2.6631854696 \\ \mathrm{Cl} & 1.9954468963 \\ \mathrm{H} & 1.1767134806 \\ \mathrm{C} & -0.7445464975 \\ \mathrm{H} & -2.7010609047 \\ \mathrm{H} & 0.4016544425 \\ \mathrm{H} & -1.7990746785 \\ \mathrm{H} & -0.4092703015 \\ \mathrm{H} & -1.0717085377 \\ \mathrm{H} & 2.6545350565 \\ \mathrm{H} & 1.7291724316 \\ \mathrm{H} & 2.1805900196 \\ \mathrm{H} & 1.3719442758 \\ \mathrm{H} & 0.9270394507 \\ \mathrm{H} & -0.3212510052 \\ \mathrm{C} & -0.2338291892 \\ \mathrm{H} & -1.8034844398 \\ \mathrm{H} & -0.8595201062 \\ \mathrm{H} & 0.8256059521 \\ & \end{array}$

1. 7493422958

2. 2438490065

1. 2055262149

3. 6732928956

3. 3681180844

1.8768531031

1.7686803540

0.0137844496

4. 3258040039

3. 9244343654

3. 5953062333

1. 3795711295

3. 9867719639

$-1.4467837684$

$-0.2766655425$

$-1.6729029917$

1.4917898085

1.3465999640

$-0.6035031312$

$-1.6695802763$

$-1.8890985917$
1.4921451161

2. 6684337961

0.2917738851

1. 2603880581

2. 6451038771

3. 1791007089

4.0865275174

$-1.0715393499$

1.3583106055

0.4641256948

2. 4982499725

3. 1785801139

3.3918212597

$-0.4508078543$

$-1.6964984756$

$-1.3957379139$

$-1.3460964296$

0.1349872670

2.2143409205

2. 0214885897

0.9580918306
0.4379394240

$-0.1774542697$

1. 2876747930

$-0.3878304258$

$-2.0246362524$

$-3.2761202046$

$-3.0061662955$

$-2.9716178789$

$-1.6079764993$

$-1.9008540447$

$-3.5356839171$

$-4.0216305945$

$-3.0619436233$

$-3.1758791230$

0.7305830042

1.2900618287

$-0.3615559507$

$-0.7414504715$

$-1.1325176448$

0.5606630772

1. 0328791618

2. 1492190108

1. 5458725381

$-4.9884555087$

$-3.7831574468$

$-5.5581931110$

$-5.2366099087$
$-1.0414368742$

0.2432570113

1.3192848353

$-0.6908698282$

1. 6416429754

0.3678704679

3. 5964378641

3.1667911985

0.7626114205

2. 3738226778

0.3275705232

$-0.5287237903$

3. 0522186505

4.1892898482

$-0.5778759966$

$-1.5669163704$

$-1.7550356544$

$-0.0094997607$

$-1.4791850866$

$-1.1484411830$

2.0533931708

0.7243140916

1. 8460651581

$-1.3204071665$

$-0.5507615466$

$-2.0025770913$

$-1.3022513616$

\section{$20\left(1^{s t}\right)$}

$\begin{array}{lrrr}\mathrm{C} & -0.5291744548 & -1.0903196159 & -1.1277770032 \\ \mathrm{P} & 0.6734668761 & -0.0657347503 & -0.1823867380 \\ \mathrm{C} & 2.2578313993 & -0.9920242109 & -0.2897007979\end{array}$




$\begin{array}{lrrr}\mathrm{C} & 0.9452089942 & 1.4183986527 & -1.2353462545 \\ \mathrm{Ru} & -0.1093081107 & 0.3337417660 & 2.0464577900 \\ \mathrm{C} & 1.2968686886 & 0.8383866625 & 3.2084858166 \\ \mathrm{C} & 2.2630737153 & -0.0212076343 & 3.8743026756 \\ \mathrm{C} & 3.4090324040 & 0.4845860114 & 4.3685145509 \\ \mathrm{C} & -0.4899980461 & 0.6941624013 & 4.2958688143 \\ \mathrm{C} & -1.3853857450 & 0.0295400046 & 3.7053329680 \\ \mathrm{Cl} & 0.1748207633 & -2.1362044762 & 2.2761080671 \\ \mathrm{Cl} & -0.6681695532 & 2.6293261625 & 1.5551941244 \\ \mathrm{H} & -0.1551144716 & -1.2899226034 & -2.1383636346 \\ \mathrm{H} & 1.5001031481 & 1.9149765045 & 3.2756996041 \\ \mathrm{H} & -0.1826860538 & 1.3525275744 & 5.0874237739 \\ \mathrm{H} & -2.3280975088 & -0.4873229062 & 3.7029595169 \\ \mathrm{H} & 2.0548417273 & -1.0863745641 & 3.9020691458 \\ \mathrm{H} & 4.1697721294 & -0.1535185746 & 4.8102883759 \\ \mathrm{H} & 3.6258184073 & 1.5505516265 & 4.3283224779 \\ \mathrm{H} & 1.7165716457 & 2.0489859102 & -0.7844104510 \\ \mathrm{H} & 1.2545479159 & 1.1262335009 & -2.2454278966 \\ \mathrm{H} & 0.0247465113 & 2.0053727658 & -1.2743997954 \\ \mathrm{H} & -1.4863888967 & -0.5642094358 & -1.2003743785 \\ \mathrm{H} & -0.6786175073 & -2.0317703591 & -0.5928403078 \\ \mathrm{H} & 2.1557522504 & -1.9181552328 & 0.2804293684 \\ \mathrm{H} & 2.5099748065 & -1.2114416466 & -1.3334384275 \\ \mathrm{H} & 3.0581945368 & -0.3920553508 & 0.1544395019\end{array}$

\section{$21\left(^{\text {st }}\right)$}

$\begin{array}{lrrr}\mathrm{C} & -3.6302500914 & 1.6577806744 & 1.0863723511 \\ \mathrm{P} & -2.4029972646 & 1.1127050130 & 2.3425844816 \\ \mathrm{Ru} & -3.1735113678 & 0.9424784444 & 4.4356664604 \\ \mathrm{C} & -3.5930312966 & 2.7299940763 & 4.6503260290 \\ \mathrm{C} & -0.9719659732 & 2.2470224571 & 2.1259907215 \\ \mathrm{C} & -1.7995196846 & -0.5142205961 & 1.7323148206 \\ \mathrm{Cl} & -1.0116936670 & 0.7157789825 & 5.3856690280 \\ \mathrm{Cl} & -5.1694264689 & -0.1496392073 & 3.7851028617 \\ \mathrm{C} & -3.9830839682 & 3.2213929232 & 5.9476799574 \\ \mathrm{H} & -3.6867572376 & 3.4353468482 & 3.8158475137 \\ \mathrm{H} & -3.9827785901 & 2.6654981004 & 1.3266610576 \\ \mathrm{H} & -4.4863402660 & 0.9790691092 & 1.1177768896 \\ \mathrm{H} & -3.1861961889 & 1.6620490958 & 0.0843892225 \\ \mathrm{H} & -1.0009221595 & -0.8663194166 & 2.3920424902 \\ \mathrm{H} & -2.6225472765 & -1.2342061476 & 1.7600589990 \\ \mathrm{H} & -1.4184117136 & -0.4328571885 & 0.7079060103 \\ \mathrm{H} & -1.2674477033 & 3.2663244838 & 2.3921199541 \\ \mathrm{H} & -0.1736440123 & 1.9348310079 & 2.8041110858 \\ \mathrm{H} & -0.6154697431 & 2.2326800930 & 1.0897121830 \\ \mathrm{C} & -3.5115045314 & 2.7436103657 & 7.1363451436 \\ \mathrm{H} & -4.7050832698 & 4.0424658675 & 5.9668399339 \\ \mathrm{C} & -3.9450870254 & 3.2246858723 & 8.4290072147 \\ \mathrm{H} & -2.7333769295 & 1.9809126389 & 7.1246008187 \\ \mathrm{C} & -3.4426316262 & 2.7674745722 & 9.5906800174 \\ \mathrm{H} & -4.7213986824 & 3.9894386188 & 8.4400655273 \\ \mathrm{H} & -3.7923136934 & 3.1377715894 & 10.5496410177 \\ \mathrm{H} & -2.6657478906 & 2.0064955483 & 9.6103936760\end{array}$




\section{$22\left(1^{\text {st }}\right)$}

$\begin{array}{lrrr}\mathrm{C} & 0.2529943065 & -0.7073137018 & -1.4319294533 \\ \mathrm{P} & 0.4017847798 & -0.1763875613 & 0.3326481644 \\ \mathrm{C} & 1.3811012538 & 1.3757128764 & 0.2116726127 \\ \mathrm{H} & 0.9053778707 & 2.0644451728 & -0.4958228165 \\ \mathrm{Ru} & 1.2935554393 & -1.9089527444 & 1.5930597883 \\ \mathrm{C} & 2.0859132820 & -2.6675991460 & 3.4456769169 \\ \mathrm{C} & 1.2556438835 & -3.8472370305 & 3.6441494249 \\ \mathrm{C} & 0.3438870696 & -3.9519710309 & 4.6352288842 \\ \mathrm{Cl} & 1.3013754443 & -3.6200106316 & -0.0049247189 \\ \mathrm{Cl} & 0.9750278287 & 0.0256054557 & 3.2172142135 \\ \mathrm{C} & 3.3093808280 & -1.6515956023 & 1.5214285056 \\ \mathrm{C} & 3.3903201952 & -2.7375305809 & 2.6267741954 \\ \mathrm{H} & 2.1006422401 & -1.9583635005 & 4.2654475565 \\ \mathrm{H} & 3.5311227785 & -3.7295472492 & 2.1889642901 \\ \mathrm{H} & 3.6405032820 & -0.6689935633 & 1.8650731296 \\ \mathrm{H} & 3.7792135539 & -1.9451455329 & 0.5802316344 \\ \mathrm{H} & 4.2248640518 & -2.5254317173 & 3.3132072362 \\ \mathrm{H} & 1.3827039709 & -4.6713912049 & 2.9432352099 \\ \mathrm{H} & -0.2616519001 & -4.8456287177 & 4.7537590526 \\ \mathrm{H} & 0.1833540202 & -3.1442953753 & 5.3449791703 \\ \mathrm{H} & 1.4542293776 & 1.8353982457 & 1.1984242425 \\ \mathrm{H} & 2.3872223987 & 1.1342279354 & -0.1436015717 \\ \mathrm{H} & 1.2333390849 & -1.0027965692 & -1.8124624309 \\ \mathrm{H} & -0.4082230777 & -1.5737886119 & -1.5029818554 \\ \mathrm{H} & -0.1456243552 & 0.1177499583 & -2.0345164124 \\ \mathrm{C} & -1.3096901432 & 0.3552533469 & 0.7449746180 \\ \mathrm{H} & -1.3229597114 & 0.7542736993 & 1.7602917547 \\ \mathrm{H} & -1.6529190571 & 1.1162266693 & 0.0343463575 \\ \mathrm{H} & -1.9784953894 & -0.5096131061 & 0.6930900323\end{array}$

\section{$23\left(1^{\text {st }}\right)$}

$\begin{array}{lrrr}\mathrm{C} & -1.0139443684 & 0.0407984554 & -0.5107889758 \\ \mathrm{P} & 0.4507819065 & -0.3562550158 & 0.5326467686 \\ \mathrm{C} & 1.7374299708 & -0.8945582227 & -0.6682863754 \\ \mathrm{H} & 1.3602144018 & -1.7040608480 & -1.3030800966 \\ \mathrm{Ru} & 1.0049775991 & 1.5218271701 & 1.9376707697 \\ \mathrm{C} & 0.7864298026 & 3.6054974734 & 2.9116581019 \\ \mathrm{C} & 1.8243846571 & 2.8892245236 & 3.4981011517 \\ \mathrm{C} & 2.8757353694 & 2.4305563346 & 2.6448075511 \\ \mathrm{C} & 3.5965730213 & 1.1553273431 & 3.0064648915 \\ \mathrm{C} & 2.4166271199 & 0.1905248928 & 2.8825244152 \\ \mathrm{Cl} & -0.6287842575 & 0.7421057988 & 3.5366589793 \\ \mathrm{Cl} & 1.6869267549 & 2.5362180418 & -0.1730424111 \\ \mathrm{H} & 3.2605763407 & 3.0821925935 & 1.8656600413 \\ \mathrm{H} & 4.0605721670 & 1.1779776960 & 4.0039515381 \\ \mathrm{H} & 2.6234194775 & -0.6764144676 & 2.2535282777 \\ \mathrm{H} & 1.9681463294 & -0.1218474535 & 3.8261410194 \\ \mathrm{H} & 4.3799073303 & 0.9413201093 & 2.2737217342 \\ \mathrm{H} & 1.6812759779 & 2.4449234782 & 4.4803711276 \\ \mathrm{H} & -0.1416359232 & 3.7498521859 & 3.4555008316 \\ \mathrm{H} & 0.9470591189 & 4.1999674134 & 2.0164043767 \\ \mathrm{H} & -1.8623765558 & 0.2791624342 & 0.1379214330 \\ \mathrm{H} & -0.7820195187 & 0.9136516815 & -1.1267432810 \\ \mathrm{H} & -1.2778336151 & -0.8070844953 & -1.1528198607 \\ \mathrm{H} & 2.0254058702 & -0.0376586946 & -1.2814926756\end{array}$




$\begin{array}{lr}\mathrm{H} & 2.6207780113 \\ \mathrm{C} & -0.0300252143 \\ \mathrm{H} & 0.8191874246 \\ \mathrm{H} & -0.8362874240 \\ \mathrm{H} & -0.3523794400\end{array}$

\section{$24\left(1^{\text {st }}\right)$}

$\begin{array}{lr}\mathrm{P} & -0.0881869670 \\ \mathrm{C} & 0.7526356128 \\ \mathrm{H} & 0.3661928816 \\ \mathrm{Ru} & 0.7343181138 \\ \mathrm{Cl} & -0.4934480746 \\ \mathrm{Cl} & 2.4108781181 \\ \mathrm{C} & -0.8251300932 \\ \mathrm{C} & -0.7821938927 \\ \mathrm{C} & -0.6268963326 \\ \mathrm{H} & -1.6098627648 \\ \mathrm{H} & -0.6342218936 \\ \mathrm{H} & -1.0615830811 \\ \mathrm{H} & 0.0067447132 \\ \mathrm{H} & 1.8292595491 \\ \mathrm{H} & 0.5813439478 \\ \mathrm{C} & -1.8768025097 \\ \mathrm{C} & 0.1341728262 \\ \mathrm{H} & -0.4428978875 \\ \mathrm{H} & 1.1886390602 \\ \mathrm{H} & -0.2000674956 \\ \mathrm{H} & -2.4882083317 \\ \mathrm{H} & -2.1418307689 \\ \mathrm{H} & -2.0823760594\end{array}$

\section{$25\left(1^{\text {st }}\right)$}

$\begin{array}{lr}\mathrm{C} & -1.3759583425 \\ \mathrm{P} & 0.1009369324 \\ \mathrm{C} & 1.3764963647 \\ \mathrm{Ru} & -0.3163840694 \\ \mathrm{C} & -2.0337598254 \\ \mathrm{C} & -1.6170216983 \\ \mathrm{C} & -0.4467488379 \\ \mathrm{C} 1 & -1.8571211554 \\ \mathrm{Cl} & 1.9158160370 \\ \mathrm{H} & -0.7076964879 \\ \mathrm{H} & -2.0001638913 \\ \mathrm{H} & -2.8970871196 \\ \mathrm{H} & 0.4652804612 \\ \mathrm{H} & 1.5427316883 \\ \mathrm{H} & 1.0608029707 \\ \mathrm{H} & 2.3063945550 \\ \mathrm{H} & -1.7726430252 \\ \mathrm{H} & -2.1421201007 \\ \mathrm{H} & -1.1166100599 \\ \mathrm{C} & 0.7295873990 \\ \mathrm{H} & -0.0010444777 \\ \mathrm{H} & 0.9022064574 \\ \mathrm{H} & 1.6698434399\end{array}$

$$
\begin{aligned}
& -1.2498172954 \\
& -1.9080323481 \\
& -2.2947456359 \\
& -1.6853629788 \\
& -2.6683932695
\end{aligned}
$$

$\begin{array}{rr}-0.3944758188 & 0.0890113848 \\ -1.6783589621 & -0.9364251385 \\ -1.6771743765 & -1.9624122912 \\ -0.4117265969 & 2.1574712169 \\ -2.4537862758 & 2.7249982521 \\ 1.2939876113 & 1.9016764757 \\ 0.3670494801 & 4.2383588762 \\ 1.5415422239 & 4.6709420962 \\ 1.0516709144 & 2.7920233833 \\ 1.1903901688 & 2.3378241773 \\ 2.5539972229 & 4.9820124433 \\ -0.6798133281 & 4.3799960643 \\ 1.9395707594 & 2.6989892103 \\ -1.4827161763 & -0.9602543278 \\ -2.6594558396 & -0.4837086676 \\ -0.7896054658 & -0.1195742591 \\ 1.1592245963 & -0.8776079857 \\ 1.9665502032 & -0.4158211104 \\ 1.4448287135 & -0.8525978818 \\ 1.0278105508 & -1.9132793023 \\ -0.0141429845 & 0.3527508795 \\ -0.8503274918 & -1.1813329796 \\ -1.7408754215 & 0.3776793490\end{array}$
0.8048715572

0.5567911471

1. 6894596352

0.7853175304

1. 7024219114

2. 9671613037

2. 9359627355

$-1.0285808876$

1. 2159695401

3.1616178443

3.8328648711

1. 1880056682

3. 4543948487

1. 4668578747

2. 7307105204

1. 5574459001

1. 8134625218

0.0830561441

0.6708195791

$-1.1141418729$

$-1.8630531093$

$-1.1858728598$

$-1.2949886177$
1.8434039335

2. 9071070085

2. 2155391252

5.1373597358

5.7509271450

5.7189821043

4.8050821304

4. 8751322817

5.7203235485

3. 7689358087

6.2658173951

6.1573997481

5.1060394378

1.1555580305

2.3173191857

2. 7742997044

1. 9914765092

2.1357920875

0.7870412858

2.4625346980

2.7763692761

1. 3824608836

2. 9920088689 


\section{$26\left(1^{\text {st }}\right)$}

$\begin{array}{lrrr}\mathrm{H} & -0.4877171065 & -0.4267001737 & -2.4874275077 \\ \mathrm{C} & -0.6811460169 & -0.0792865111 & -1.4659941499 \\ \mathrm{P} & 0.8765336262 & 0.4764243313 & -0.6564545128 \\ \mathrm{C} & 1.5734859512 & 1.7389304423 & -1.7998327994 \\ \mathrm{Ru} & 0.5744254582 & 0.9656335843 & 1.6004981515 \\ \mathrm{Cl} & 2.6719264348 & 1.0582739834 & 2.5907410536 \\ \mathrm{Cl} & -0.2528293984 & -1.2244880253 & 2.1548322218 \\ \mathrm{C} & 0.8984443631 & 3.1449985638 & 1.2475576588 \\ \mathrm{C} & -0.0285938315 & 3.1902095896 & 2.3672733644 \\ \mathrm{C} & -0.8384663365 & 2.0937735679 & 2.2930202219 \\ \mathrm{H} & -1.6954224943 & 1.7102463797 & 2.8366565684 \\ \mathrm{H} & 0.0784514787 & 3.8366388741 & 3.2447058180 \\ \mathrm{H} & 1.8937125388 & 3.5659382487 & 1.3797168495 \\ \mathrm{H} & 0.4558001394 & 3.3601902293 & 0.2790375575 \\ \mathrm{H} & 0.9031623560 & 2.5999621112 & -1.8710453150 \\ \mathrm{H} & 2.5381951633 & 2.0821152087 & -1.4154238091 \\ \mathrm{H} & 1.7112484855 & 1.3130451098 & -2.8000081611 \\ \mathrm{H} & -1.4003892753 & 0.7447625207 & -1.4957844903 \\ \mathrm{H} & -1.1053986010 & -0.8921094041 & -0.8698056582 \\ \mathrm{C} & 2.0153170382 & -0.9612848852 & -0.8143742860 \\ \mathrm{H} & 2.9886051536 & -0.7028025229 & -0.3876303152 \\ \mathrm{H} & 1.5976445077 & -1.7938740505 & -0.2417269654 \\ \mathrm{H} & 2.1367010061 & -1.2481543342 & -1.8654042933\end{array}$

\section{$27\left(1^{\text {st }}\right)$}

$\begin{array}{lrrr}\mathrm{H} & -0.8684137610 & 0.0634937603 & -2.5804379847 \\ \mathrm{C} & -1.0336718425 & 0.2373659529 & -1.5112184712 \\ \mathrm{P} & 0.5652887208 & 0.4999637583 & -0.6421240004 \\ \mathrm{C} & 1.2720934661 & 1.9565250482 & -1.5371739020 \\ \mathrm{Ru} & 0.4101373484 & 0.4939101290 & 1.7487653189 \\ \mathrm{C} 1 & 1.0307038637 & -0.2801889991 & 3.8898446701 \\ \mathrm{Cl} & -0.6320839829 & -1.6336832347 & 1.3326339884 \\ \mathrm{C} & 1.5885939498 & 2.5339800182 & 1.8770383083 \\ \mathrm{C} & 0.3533062777 & 2.5741668709 & 2.5235305687 \\ \mathrm{C} & -0.7529867994 & 1.9026177066 & 1.9067399064 \\ \mathrm{H} & -1.8310460117 & 2.0211607541 & 2.0016485882 \\ \mathrm{H} & 0.3236989732 & 2.7103096553 & 3.6080620403 \\ \mathrm{H} & 2.4920333053 & 2.6145686977 & 2.4746577713 \\ \mathrm{H} & 1.6843231346 & 2.7720367656 & 0.8279035856 \\ \mathrm{H} & 2.3091429671 & 2.1286119747 & -1.2340047703 \\ \mathrm{H} & 1.2520951939 & 1.7709860933 & -2.6168897279 \\ \mathrm{H} & 0.6883363823 & 2.8582006958 & -1.3272914288 \\ \mathrm{H} & -1.6634154213 & 1.1233758775 & -1.3831670309 \\ \mathrm{H} & -1.5340115113 & -0.6227247793 & -1.0614486324 \\ \mathrm{C} & 1.6473848520 & -0.8791861146 & -1.2046435143 \\ \mathrm{H} & 1.2383097817 & -1.8181608176 & -0.8252093598 \\ \mathrm{H} & 1.6987702283 & -0.9039627954 & -2.2991457572 \\ \mathrm{H} & 2.6558563183 & -0.7465551527 & -0.8003070013\end{array}$




\section{$28\left(1^{\text {st }}\right)$}

$\begin{array}{lrrr}\mathrm{P} & 0.1431318845 & 0.1952356944 & -0.2598461814 \\ \mathrm{C} & 1.4546543769 & 0.8630019409 & -1.3584412153 \\ \mathrm{H} & 1.0331201275 & 1.1949103489 & -2.3148080248 \\ \mathrm{Ru} & 0.7614334784 & -0.1287168130 & 2.0081649362 \\ \mathrm{Cl} & 0.2920235995 & 0.1250895473 & 4.2997284835 \\ \mathrm{Cl} & 1.5623543214 & 2.0982212112 & 1.8821230987 \\ \mathrm{C} & 2.2497710547 & -1.1465744175 & 1.7672336245 \\ \mathrm{C} & 1.6977858574 & -2.3916094551 & 2.2680751101 \\ \mathrm{C} & 0.5682290065 & -2.9530946025 & 1.7633374810 \\ \mathrm{H} & 3.3027443780 & -0.8700651685 & 1.6919879871 \\ \mathrm{H} & 2.0719108113 & -2.7419691505 & 3.2331979783 \\ \mathrm{H} & 0.0320865141 & -3.7172351801 & 2.3188425366 \\ \mathrm{H} & 0.2133215400 & -2.7243332125 & 0.7669818032 \\ \mathrm{H} & 1.9306228651 & 1.7028820900 & -0.8465007814 \\ \mathrm{H} & 2.2063345909 & 0.0861082382 & -1.5404322396 \\ \mathrm{C} & -1.1960880178 & 1.4592837367 & -0.2991714645 \\ \mathrm{C} & -0.5651798530 & -1.1918666043 & -1.2544671104 \\ \mathrm{H} & -1.4112778415 & -1.6400346591 & -0.7256687018 \\ \mathrm{H} & -0.9085334620 & -0.8206887332 & -2.2287763148 \\ \mathrm{H} & 0.1902464427 & -1.9648764193 & -1.4248726569 \\ \mathrm{H} & -2.0621710910 & 1.1021227803 & 0.2668601478 \\ \mathrm{H} & -0.8234295875 & 2.3720693568 & 0.1727735610 \\ \mathrm{H} & -1.5029803968 & 1.6667369069 & -1.3304044945\end{array}$

\section{$29\left(1^{\text {st }}\right)$}

$\begin{array}{ll}\mathrm{C} & -3.9370902520 \\ \mathrm{P} & -2.5567567622 \\ \mathrm{Ru} & -3.0882952248 \\ \mathrm{C} & -3.9659988923 \\ \mathrm{C} & -1.1587165539 \\ \mathrm{C} & -2.0229487581 \\ \mathrm{Cl} & -0.8887396009 \\ \mathrm{Cl} & -4.7947665418 \\ \mathrm{H} & -4.5329999783 \\ \mathrm{C} & -4.1072510554 \\ \mathrm{H} & -4.2659377140 \\ \mathrm{H} & -4.7690785377 \\ \mathrm{H} & -3.6153633913 \\ \mathrm{H} & -1.1342185043 \\ \mathrm{H} & -2.8249380688 \\ \mathrm{H} & -1.7833523081 \\ \mathrm{H} & -1.4402109631 \\ \mathrm{H} & -0.2999248332 \\ \mathrm{H} & -0.8971553901 \\ \mathrm{C} & -3.0838134413 \\ \mathrm{H} & -5.1255845233 \\ \mathrm{H} & -3.2513293959 \\ \mathrm{H} & -2.0560297836\end{array}$

1. 2178784771

0.8822004039

0.9970568324

2. 5956617866

1. 9040835117

$-0.8360881867$

1. 4590293826

$-0.6267346363$

2. 6157038906

3. 7907251872

2. 2538221028

0.5520217266

1. 0547072444

$-1.0841960701$

$-1.5310679263$

$-0.9246705420$

2. 9589210881

1. 7796468983

1. 5885112701

4. 4916427996

4.1754411184

5.4179247870

4.1831214414
1.0907631341

2. 2563591017

4. 4451623260

4.5505608903

1.6330785779

1.8573091661

5.1670494010

4.1624216273

5.5018966414

3. 7341429568

1. 2087134680

1. 3335154243

0.0558240017

2. 4459721388

2.1176448899

0.7913567366

1. 6149747271

2. 2971280680

0.6164805338

3. 2164621886

3. 6293694305

2. 6735680207

3. 3818433703

\section{$32\left(1^{\text {st }}\right)$}

$\begin{array}{lrrr}\mathrm{C} & -1.5030290056 & -0.0306051995 & -1.1795297517 \\ \mathrm{P} & -0.2164210455 & -0.0147168286 & 0.1378013972 \\ \mathrm{C} & 1.3505046190 & 0.2327728373 & -0.8033755783\end{array}$




$\begin{array}{lrrr}\mathrm{Ru} & -0.2695268841 & -1.9913716798 & 1.6326907799 \\ \mathrm{C} & -0.8166784265 & -4.1350911279 & 1.7787725109 \\ \mathrm{C} & -1.0530322757 & -3.2628174066 & 2.8218911236 \\ \mathrm{C} & -0.4613925592 & 1.6033010995 & 0.9861167576 \\ \mathrm{Cl} & 2.0180451858 & -2.3959369406 & 1.2591405584 \\ \mathrm{C} & -1.0631783471 & -3.5536316005 & 0.4668850210 \\ \mathrm{Cl} & -2.5729773327 & -1.0827826246 & 1.9597461108 \\ \mathrm{H} & 1.2764201805 & 1.1146058496 & -1.4501824234 \\ \mathrm{H} & -0.4662660359 & -3.9382883261 & -0.3585850763 \\ \mathrm{H} & -2.0873850389 & -3.2858017823 & 0.2156020870 \\ \mathrm{H} & -1.3331180088 & -3.3177496016 & 3.8673252291 \\ \mathrm{H} & -0.1870345763 & -5.0211099304 & 1.9156173052 \\ \mathrm{H} & -1.4520372697 & 0.8837346348 & -1.7816746165 \\ \mathrm{H} & -1.3439310747 & -0.8963727885 & -1.8295833275 \\ \mathrm{H} & -2.4869444829 & -0.1161358697 & -0.7133409185 \\ \mathrm{H} & 2.1815705127 & 0.3543188969 & -0.1042302918 \\ \mathrm{H} & 1.5569192549 & -0.6530390829 & -1.4097022534 \\ \mathrm{H} & -1.4130935458 & 1.5812720737 & 1.5224983336 \\ \mathrm{H} & 0.3455577573 & 1.7627273516 & 1.7086441487 \\ \mathrm{H} & -0.4603262742 & 2.4275941302 & 0.2638252532\end{array}$

\section{$33\left(1^{\text {st }}\right)$}

$\begin{array}{lrrr}\mathrm{C} & -1.0366706550 & -3.1971091527 & 0.5223204116 \\ \mathrm{C} & -0.7502314178 & -3.9606837289 & 1.7180775258 \\ \mathrm{C} & -0.8125903118 & -3.2471767771 & 2.9057524846 \\ \mathrm{Ru} & -0.1024076607 & -1.8476495160 & 1.8691378831 \\ \mathrm{Cl} & 2.1602002008 & -2.3222292138 & 1.4071908048 \\ \mathrm{C} 1 & -2.3732692753 & -0.8486036482 & 2.0271262762 \\ \mathrm{P} & 0.3848936574 & 0.6209710733 & 1.6036743297 \\ \mathrm{C} & -0.1221791057 & 1.6158721074 & 3.0690208205 \\ \mathrm{C} & 2.1563722643 & 1.0728750341 & 1.3661625798 \\ \mathrm{C} & -0.4579098437 & 1.4200773330 & 0.1753858274 \\ \mathrm{H} & -0.4893451682 & -3.4806152846 & -0.3750858437 \\ \mathrm{H} & -2.0385202496 & -2.8101337101 & 0.3634662691 \\ \mathrm{H} & -0.1103396689 & -4.8486363273 & 1.6714297951 \\ \mathrm{H} & -0.8106695833 & -3.5210854364 & 3.9562975246 \\ \mathrm{H} & 2.7398387332 & 0.7379277741 & 2.2280263354 \\ \mathrm{H} & 2.2649512659 & 2.1575111774 & 1.2509069608 \\ \mathrm{H} & 2.5494790208 & 0.5652652014 & 0.4819440374 \\ \mathrm{H} & -0.0758101521 & 0.9893223316 & -0.7553436432 \\ \mathrm{H} & -0.2706112095 & 2.5001936686 & 0.1725780196 \\ \mathrm{H} & -1.5297891037 & 1.2240260871 & 0.2428742990 \\ \mathrm{H} & -1.1828698758 & 1.4407504944 & 3.2620254205 \\ \mathrm{H} & 0.0576610033 & 2.6825249802 & 2.8924175744 \\ \mathrm{H} & 0.4527729890 & 1.2987317994 & 3.9449940797\end{array}$




\section{Intramolecular Enyne Metathesis}

\section{1-vinyl cyclopentene}

$\begin{array}{lr}\text { C } & 1.5249533548 \\ \text { C } & 1.3921091879 \\ \text { C } & -0.0149666564 \\ \text { C } & -0.7640146829 \\ \text { C } & 0.0907159853 \\ \text { C } & -2.2015137307 \\ \text { C } & -3.0460164672 \\ \text { H } & 0.0274927671 \\ \text { H } & -0.5027766341 \\ \text { H } & -0.2037257872 \\ \text { H } & 2.0612564205 \\ \text { H } & 2.0853689797 \\ \text { H } & 1.4406000614 \\ \text { H } & 2.1929723862 \\ \text { H } & -2.5971658241 \\ \text { H } & -4.1097284098 \\ \text { H } & -2.7045998157\end{array}$

\author{
1.2448281916 \\ $-0.0415343182$ \\ 0.0388115908 \\ 1.0132857212 \\ 1.6627746253 \\ 1.2378036437 \\ 0.6239655187 \\ 0.4257539960 \\ $-0.9422327508$ \\ 2.4355548130 \\ 1.0695276273 \\ 2.0321454751 \\ $-0.9188449198$ \\ $-0.1439442455$ \\ 1.9717564782 \\ 0.8428010544 \\ $-0.1135842112$
}

-1.3825725373
-0.5244666591
0.1215143083
-0.7737396299
-1.5878054566
-0.7098057495
0.1335142569
1.1507192264
0.1788184000
-2.2938809317
-2.3243177449
-0.8531033953
-1.1797739307
0.2147340024
-1.4123250689
0.1286599060
0.8552055980

0.2297448881

1.2361015452

$-0.0244150517$

1.8133716338

1.5216321429

$-0.6231911003$

$-2.1087404821$

$-0.5392309688$

$-0.2467356073$

$-2.9823706449$

$-2.2157690808$

$-2.4833488479$

$-4.4002481256$

$-2.8691433992$

$-2.6158553847$

$-5.5631168835$

$-6.5910107029$

34

$\begin{array}{lr}\mathrm{N} & 1.2834890000 \\ \mathrm{C} & 1.0461500000 \\ \mathrm{C} & -0.4828420000 \\ \mathrm{~N} & -0.8597380000 \\ \mathrm{C} & 0.1579750000 \\ \mathrm{C} & -2.2481990000 \\ \mathrm{Ru} & 0.0080690000 \\ \mathrm{C} & 1.2172640000 \\ \mathrm{C} & 2.6657320000 \\ \mathrm{Cl} & 1.1844840000 \\ \mathrm{Cl} & -1.9598940000 \\ \mathrm{C} & 0.0910520000 \\ \mathrm{C} & -0.7620030000\end{array}$

0.2335070000

$-0.0227120000$

$-0.0824530000$

$-0.2087900000$

0.0732860000

$-0.4931840000$

0.2017430000

1. 5641190000

0.1473590000

$-1.8852780000$

1.5907380000

0.4869460000

$-0.5566330000$
$-0.8602190000$

$-2.2891900000$

$-2.3679600000$

$-0.9557200000$

$-0.1140660000$

$-0.6337520000$

1. 9046270000

1.9540610000

$-0.4050390000$

2. 0417070000

1.8389470000

4.4961230000

4.3940400000 


$\begin{array}{lr}\mathrm{C} & -2.2581870000 \\ \mathrm{C} & -2.7244270000 \\ \mathrm{C} & -4.2636880000 \\ \mathrm{C} & -4.7381840000 \\ \mathrm{C} & -5.1053850000 \\ \mathrm{H} & 1.4787460000 \\ \mathrm{H} & 1.5163500000 \\ \mathrm{H} & -0.8526330000 \\ \mathrm{H} & -0.9181100000 \\ \mathrm{H} & 3.2251300000 \\ \mathrm{H} & 2.6998140000 \\ \mathrm{H} & 3.1423040000 \\ \mathrm{H} & -2.5735750000 \\ \mathrm{H} & -2.3484290000 \\ \mathrm{H} & -2.8930970000 \\ \mathrm{H} & 1.6107190000 \\ \mathrm{H} & 1.5555170000 \\ \mathrm{H} & -0.2724850000 \\ \mathrm{H} & -0.3381340000 \\ \mathrm{H} & 1.1624980000 \\ \mathrm{H} & -2.5878590000 \\ \mathrm{H} & -2.7357470000 \\ \mathrm{H} & -2.3682770000 \\ \mathrm{H} & -2.2859490000 \\ \mathrm{H} & -4.6264580000 \\ \mathrm{H} & -4.7031810000 \\ \mathrm{H} & -5.4338020000\end{array}$

-0.4744760000
-1.0651120000
-1.0470050000
-1.6038410000
-2.0693000000
0.7709810000
-0.9747530000
-0.9358270000
0.8338850000
1.0441780000
0.0196380000
-0.7275250000
-1.3498290000
-0.7394710000
0.3667040000
1.9496590000
2.1016580000
1.5025490000
-1.5565410000
0.3189280000
0.5641650000
-1.0371090000
-2.0976240000
-0.4940740000
-0.0151040000
-1.6127860000
-2.4788760000

4.5286980000

5.8758890000

6.0009720000

7.2699640000

8. 3227140000

$-2.9071310000$

$-2.5742970000$

$-2.9462640000$

$-2.7905540000$

$-0.6993740000$

0.6734920000

$-0.8663180000$

$-1.2368890000$

0.4205280000

$-0.8450880000$

2. 9014470000

1.0622180000

4. 6319840000

4. 3113300000

4.5501870000

4.4280460000

3. 7140650000

5.9819240000

6.7030150000

5.8944620000

5.1672550000

9. 2502170000

35

$\begin{array}{lrrr}\mathrm{C} & -1.4873536837 & -0.3483001337 & -1.9137674118 \\ \mathrm{~N} & -1.5207506971 & 0.0109258129 & -0.4885881183 \\ \mathrm{C} & -0.2834912050 & 0.1617836622 & 0.0259125166 \\ \mathrm{~N} & 0.6180499811 & -0.1831390141 & -0.9133548767 \\ \mathrm{C} & -0.0289450612 & -0.7803085794 & -2.0948302917 \\ \mathrm{C} & -2.7733941506 & 0.4782933510 & 0.0817921643 \\ \mathrm{Ru} & 0.0322157456 & 0.7011547289 & 1.9936623486 \\ \mathrm{Cl} & -0.8827353598 & 2.9248851038 & 1.4661871934 \\ \mathrm{C} & 2.0337949292 & -0.4436282539 & -0.6885589723 \\ \mathrm{C} & 0.0729141683 & 1.6470202183 & 4.2947916058 \\ \mathrm{C} & -0.5857005566 & 0.4500054748 & 4.2814009372 \\ \mathrm{C} & -2.0787874250 & 0.3050295059 & 4.0989251898 \\ \mathrm{C} & -2.7526235194 & -0.2078496258 & 5.3882662498 \\ \mathrm{C} & -4.2623463683 & -0.4545187826 & 5.1848900374 \\ \mathrm{C} & -4.9162749737 & -0.9696972829 & 6.3894418650 \\ \mathrm{C} & -5.4248285380 & -1.3993877174 & 7.3973783995 \\ \mathrm{C} & 1.7386523765 & 1.3112413323 & 1.9138615158 \\ \mathrm{Cl} & 0.7705534354 & -1.6265892282 & 2.3467972930 \\ \mathrm{H} & 0.4219813308 & -0.4059427550 & -3.0186329673 \\ \mathrm{H} & 0.0840822463 & -1.8731709018 & -2.0660230046 \\ \mathrm{H} & -2.2064294101 & -1.1430249876 & -2.1354120743 \\ \mathrm{H} & -1.7413848421 & 0.5325409421 & -2.5211229502 \\ \mathrm{H} & 2.5138885638 & -0.5680716114 & -1.6633702148 \\ \mathrm{H} & 2.4941826672 & 0.4106201912 & -0.1890476142 \\ \mathrm{H} & 2.1802563942 & -1.3375163265 & -0.0738876995 \\ \mathrm{H} & -3.5159568737 & -0.3279592465 & 0.0457084170 \\ \mathrm{H} & -2.6236640859 & 0.7974140935 & 1.1108068163 \\ \mathrm{H} & -3.1496821227 & 1.3436294949 & -0.4776174339\end{array}$




$\begin{array}{lrrr}\mathrm{H} & 1.9578021319 & 2.3495570216 & 1.6352302533 \\ \mathrm{H} & 2.5850472136 & 0.6755176891 & 2.2014199322 \\ \mathrm{H} & -0.4358737293 & 2.5865253381 & 4.1041290451 \\ \mathrm{H} & -0.0563691154 & -0.4505137140 & 4.5805512710 \\ \mathrm{H} & 1.1073005317 & 1.7000868078 & 4.6212185239 \\ \mathrm{H} & -2.5182990730 & 1.2623751575 & 3.7932076658 \\ \mathrm{H} & -2.2818735707 & -0.4292696645 & 3.3022465177 \\ \mathrm{H} & -2.2763249445 & -1.1426561776 & 5.7068421471 \\ \mathrm{H} & -2.6054272871 & 0.5180100745 & 6.1965227152 \\ \mathrm{H} & -4.7492909538 & 0.4801269976 & 4.8726439314 \\ \mathrm{H} & -4.4051095976 & -1.1674311125 & 4.3601727768 \\ \mathrm{H} & -5.8812789353 & -1.7790810421 & 8.2824788898\end{array}$

36

\begin{tabular}{|c|c|c|c|}
\hline $\mathrm{N}$ & -1.3221096096 & -0.1563232372 & -0.7082645705 \\
\hline $\mathrm{C}$ & -0.1664279493 & 0.1953544796 & -0.1103357479 \\
\hline $\mathrm{N}$ & 0.8135759316 & 0.2033258807 & -1.0342022904 \\
\hline $\mathrm{C}$ & 0.3484559636 & -0.3468794641 & -2.3194405283 \\
\hline $\mathrm{C}$ & -1.1746114162 & -0.2771425828 & -2.1660253987 \\
\hline $\mathrm{Ru}$ & -0.0501061253 & 0.5089616997 & 1.9208745346 \\
\hline $\mathrm{C}$ & 0.1071670000 & 1.0356400000 & 4.3098610000 \\
\hline $\mathrm{C}$ & -0.7784629000 & 0.0030565799 & 4.0682718496 \\
\hline $\mathrm{C}$ & -2.2819608987 & 0.1734115917 & 4.0422299193 \\
\hline $\mathrm{C}$ & -2.9025875775 & -0.1725361922 & 5.4120684687 \\
\hline $\mathrm{C}$ & -4.4415098631 & -0.0540414028 & 5.3785147082 \\
\hline $\mathrm{C}$ & -5.0614353256 & -0.3500308573 & 6.6717333850 \\
\hline $\mathrm{C}$ & -5.5454510556 & -0.5968276963 & 7.7507550725 \\
\hline $\mathrm{C}$ & 2.2433192324 & 0.2152212952 & -0.7666976624 \\
\hline $\mathrm{C}$ & -2.6556772802 & -0.0409879831 & -0.1412341264 \\
\hline $\mathrm{C}$ & 1.4717190000 & 1.4611430000 & 2.2571640000 \\
\hline $\mathrm{Cl}$ & 1.1241378233 & -1.6406350547 & 2.0682887813 \\
\hline $\mathrm{Cl}$ & -1.3301182965 & 2.5919980633 & 1.6785317071 \\
\hline $\mathrm{H}$ & 0.7284090653 & 0.2436905517 & -3.1583714762 \\
\hline $\mathrm{H}$ & 0.7049011459 & -1.3812139746 & -2.4279335740 \\
\hline $\mathrm{H}$ & -1.6901468857 & -1.1666556659 & -2.5410385389 \\
\hline $\mathrm{H}$ & -1.6032369847 & 0.6091099013 & -2.6561225204 \\
\hline $\mathrm{H}$ & 2.7596542315 & 0.5981068126 & -1.6528074071 \\
\hline $\mathrm{H}$ & 2.4503561836 & 0.8741624511 & 0.0749853798 \\
\hline $\mathrm{H}$ & 2.6097891715 & -0.7893483868 & -0.5249223448 \\
\hline $\mathrm{H}$ & -3.2427838752 & -0.9260908251 & -0.4114758135 \\
\hline $\mathrm{H}$ & -2.5984268277 & 0.0334530132 & 0.9437502904 \\
\hline $\mathrm{H}$ & -3.1597683598 & 0.8614356704 & -0.5080490411 \\
\hline $\mathrm{H}$ & 1.4643863688 & 2.5571618479 & 2.2596148757 \\
\hline $\mathrm{H}$ & 2.4167048507 & 0.9629048541 & 2.5048038640 \\
\hline $\mathrm{H}$ & -0.2384215531 & 2.0638171964 & 4.3691871344 \\
\hline $\mathrm{H}$ & -0.4095245476 & -1.0130476372 & 4.1938978532 \\
\hline $\mathrm{H}$ & 1.1058950144 & 0.8161484496 & 4.6745035826 \\
\hline $\mathrm{H}$ & -2.5397772096 & 1.1974266543 & 3.7542012741 \\
\hline $\mathrm{H}$ & -2.7187397172 & -0.4989823811 & 3.2885169959 \\
\hline $\mathrm{H}$ & -2.6244988812 & -1.1905881515 & 5.7113642624 \\
\hline $\mathrm{H}$ & -2.5029255858 & 0.5038166441 & 6.1770320949 \\
\hline $\mathrm{H}$ & -4.7214586849 & 0.9604832635 & 5.0619403397 \\
\hline $\mathrm{H}$ & -4.8436631650 & -0.7356584388 & 4.6154866288 \\
\hline 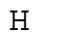 & -5.9813662483 & -0.8130979757 & 8.6989594865 \\
\hline
\end{tabular}




\begin{tabular}{|c|c|c|c|}
\hline $\mathrm{N}$ & -0.5308996156 & -0.8565008572 & -2.1809782639 \\
\hline $\mathrm{C}$ & -0.0079881762 & 0.0736457942 & -1.3666293507 \\
\hline $\mathrm{N}$ & 0.5141038432 & 1.0730322834 & -2.091829095 \\
\hline $\mathrm{C}$ & 0.5108454898 & 0.7416210433 & -3.52660597 \\
\hline $\mathrm{C}$ & -0.5156608594 & -0.3999172639 & -3.58105489 \\
\hline $\mathrm{Ru}$ & 0.0259574425 & -0.0270014999 & 0.657495 \\
\hline $\mathrm{Cl}$ & -2.2816832864 & 0.7797626984 & 0.622 \\
\hline $\mathrm{C}$ & 1.4023530029 & 2.1017986579 & -1.5674943 \\
\hline $\mathrm{C}$ & -1.4415198293 & -1.9114827363 & -1.761007 \\
\hline $\mathrm{C}$ & 0.4543086355 & 1.2132375390 & 2.13 \\
\hline $\mathrm{C}$ & 0.0649185399 & -0.0914461980 & 2.9307 \\
\hline $\mathrm{C}$ & -0.5187824796 & -1.3115967268 & 2.07 \\
\hline $\mathrm{Cl}$ & 2.3714522145 & -0.7016431590 & 0.51 \\
\hline $\mathrm{H}$ & 0.2256650820 & 1.6096549003 & -4.1279 \\
\hline $\mathrm{H}$ & 1.5126787891 & 0.4099803192 & -3.8343 \\
\hline $\mathrm{H}$ & -0.2252610124 & -1.2116880231 & -4.2542 \\
\hline $\mathrm{H}$ & -1.5156699974 & -0.0429430538 & -3.8660 \\
\hline $\mathrm{H}$ & 1.0883242687 & 2.3672117955 & -0.5565 \\
\hline $\mathrm{H}$ & 2.4403104922 & 1.7509193983 & -1.53 \\
\hline $\mathrm{H}$ & 1.3258057897 & 2.9904340117 & -2.2016 \\
\hline $\mathrm{H}$ & -1.2990318767 & -2.7859409187 & -2.4 \\
\hline $\mathrm{H}$ & -1.2193841082 & -2.1933481808 & -0.7310 \\
\hline $\mathrm{H}$ & -2.4848383114 & -1.5770466153 & -1.81 \\
\hline $\mathrm{H}$ & 1.5084892875 & 1.4720150238 & 2.2343747 \\
\hline $\mathrm{H}$ & -0.2478240529 & 2.0348735193 & 2.2755 \\
\hline $\mathrm{H}$ & -0.7449719813 & 0.1929036167 & 3.60556 \\
\hline $\mathrm{H}$ & -1.6059376863 & -1.3591129129 & 2.1776 \\
\hline $\mathrm{C}$ & 0.1645401241 & -2.6534261164 & 2.2591 \\
\hline $\mathrm{H}$ & 0.9551675499 & -0.4533937059 & 3.446 \\
\hline $\mathrm{C}$ & -0.1977959365 & -3.3206613727 & 3.60106494 \\
\hline $\mathrm{H}$ & -0.1557087166 & -3.3168713474 & 1.4423 \\
\hline $\mathrm{H}$ & 1.2483572244 & -2.5409193016 & 2.16424328 \\
\hline $\mathrm{C}$ & 0.4760279930 & -4.7030112573 & 3.74367370 \\
\hline $\mathrm{H}$ & 0.1180820096 & -2.6842293175 & 4.43710478 \\
\hline $\mathrm{H}$ & -1.2855140674 & -3.4378243953 & 3.68795150 \\
\hline $\mathrm{C}$ & 0.1692737483 & -5.3579658637 & 5.01723656 \\
\hline $\mathrm{H}$ & 0.1599993073 & -5.3504381586 & 2.91342321 \\
\hline $\mathrm{H}$ & 1.5644427771 & -4.5882241484 & 3.64411146 \\
\hline $\mathrm{C}$ & -0.0984158665 & -5.8735473217 & 6.0766 \\
\hline $\mathrm{H}$ & -0.3286497943 & -6.3362422921 & 7.0086 \\
\hline
\end{tabular}

\section{7 diastereomer}

C $\quad-0.5762335874$

$\mathrm{N} \quad-0.6034047956$

C $\quad-0.0206012708$

$\mathrm{N} \quad 0.5530419314$

0.5134410618

$-1.5629520811$

$\mathrm{Ru} \quad-0.0193600063$

Cl 2.2838210627

C $\quad 1.5215365570$

Cl $\quad-2.2699641988$

C $\quad 0.5497341493$

C $\quad 0.0517660166$

C $\quad-0.3081308857$
-0.4566827586
-0.8689377180
0.0532533262
0.9979184412
0.6259189702
-1.8592868418
0.0245885663
-0.7997581588
1.9755815212
0.9590123260
1.2539590702
0.0123532539
-1.3096790147

$-3.5328230417$

$-2.1194625842$

$-1.3377890484$

$-2.0962695289$

$-3.5208796364$

$-1.6542101894$

0.6903893834

0.5244465437

$-1.6178930105$

0.6695136159

2.1344001911

2. 9648979171

2.1416302190 


$\begin{array}{lrrr}\mathrm{C} & -1.6534038326 & -1.9443044928 & 2.4347051318 \\ \mathrm{C} & -1.6546611323 & -2.7022502426 & 3.7774937236 \\ \mathrm{C} & -2.9947946415 & -3.4298909740 & 4.0206268061 \\ \mathrm{C} & -3.0193745695 & -4.1671161888 & 5.2859983764 \\ \mathrm{C} & -3.0090892426 & -4.7676851649 & 6.3342779736 \\ \mathrm{H} & 0.2716401527 & 1.4912481960 & -4.1445821456 \\ \mathrm{H} & 1.4920947516 & 0.2296969309 & -3.8269673611 \\ \mathrm{H} & -0.3387142625 & -1.3034182277 & -4.1832406492 \\ \mathrm{H} & -1.5567492058 & -0.0515993707 & -3.8210158520 \\ \mathrm{H} & 1.2823248303 & 2.2485702450 & -0.5896504265 \\ \mathrm{H} & 2.5384836298 & 1.5668901172 & -1.6431502854 \\ \mathrm{H} & 1.4603905189 & 2.8709210755 & -2.2445691711 \\ \mathrm{H} & -1.5280775524 & -2.7282664980 & -2.3187933896 \\ \mathrm{H} & -1.2898777556 & -2.1813083988 & -0.6481055539 \\ \mathrm{H} & -2.5794576919 & -1.4484508820 & -1.6290619608 \\ \mathrm{H} & 1.6211157307 & 1.4323451986 & 2.2148155032 \\ \mathrm{H} & -0.0850742720 & 2.1321464945 & 2.2526860921 \\ \mathrm{H} & -0.8373383745 & 0.3048344838 & 3.5246729148 \\ \mathrm{H} & 0.5210297796 & -2.0200079238 & 2.1821064074 \\ \mathrm{H} & 0.8909267981 & -0.2770594102 & 3.6010024995 \\ \mathrm{H} & -1.8765238893 & -2.6645687848 & 1.6339612802 \\ \mathrm{H} & -2.4452156899 & -1.1896044807 & 2.4109860286 \\ \mathrm{H} & -0.8392675367 & -3.4364595699 & 3.7975427087 \\ \mathrm{H} & -1.4716333818 & -2.0061429266 & 4.6055833493 \\ \mathrm{H} & -3.8153048607 & -2.6990266465 & 4.0048309456 \\ \mathrm{H} & -3.1873877117 & -4.1244063818 & 3.1904845106 \\ \mathrm{H} & -3.0093012233 & -5.3003216892 & 7.2574010820\end{array}$

38

$\begin{array}{rrrr}\mathrm{C} & 0.072628 & 0.772134 & -2.341415 \\ \mathrm{C} & -1.048478 & -0.272148 & -2.244688 \\ \mathrm{~N} & -0.942255 & -0.690101 & -0.840100 \\ \mathrm{C} & -0.233197 & 0.186761 & -0.103538 \\ \mathrm{~N} & 0.315096 & 1.100799 & -0.925840 \\ \mathrm{C} & -1.863383 & -1.676952 & -0.296004 \\ \mathrm{Ru} & -0.104756 & -0.035910 & 1.935157 \\ \mathrm{C} & 0.165873 & -0.224073 & 4.309359 \\ \mathrm{C} & -0.487763 & -1.300010 & 3.706272 \\ \mathrm{C} & 1.392880 & 2.015882 & -0.599784 \\ \mathrm{Cl} & 2.066632 & -1.116829 & 1.653455 \\ \mathrm{Cl} & -2.429180 & 0.771846 & 2.112380 \\ \mathrm{C} & 0.684355 & 1.435612 & 2.765525 \\ \mathrm{C} & 0.060562 & 2.738216 & 3.175319 \\ \mathrm{C} & 0.725734 & 3.399820 & 4.395134 \\ \mathrm{C} & 0.096413 & 4.776821 & 4.698698 \\ \mathrm{C} & 0.688308 & 5.427468 & 5.869349 \\ \mathrm{C} & 1.193026 & 5.940354 & 6.839695 \\ \mathrm{H} & -0.214048 & 1.664638 & -2.906036 \\ \mathrm{H} & 0.989008 & 0.356615 & -2.784695 \\ \mathrm{H} & -0.907011 & -1.122483 & -2.918814 \\ \mathrm{H} & -2.039604 & 0.165358 & -2.431721 \\ \mathrm{H} & 1.188968 & 3.001201 & -1.034531 \\ \mathrm{H} & 1.473230 & 2.104364 & 0.481448 \\ \mathrm{H} & 2.349372 & 1.642623 & -0.989406 \\ \mathrm{H} & -1.902548 & -2.536790 & -0.972967 \\ \mathrm{H} & -1.499780 & -2.028718 & 0.672568 \\ \mathrm{H} & -2.866194 & -1.258241 & -0.156446\end{array}$




$\begin{array}{rrrr}\mathrm{H} & 1.774333 & 1.378330 & 2.905443 \\ \mathrm{H} & -0.423199 & 0.511802 & 4.847844 \\ \mathrm{H} & -1.570039 & -1.367435 & 3.772520 \\ \mathrm{H} & 0.076536 & -2.203942 & 3.491461 \\ \mathrm{H} & 1.221506 & -0.305391 & 4.547986 \\ \mathrm{H} & 0.175435 & 3.405551 & 2.302346 \\ \mathrm{H} & -1.018416 & 2.620670 & 3.309865 \\ \mathrm{H} & 0.619348 & 2.758399 & 5.278531 \\ \mathrm{H} & 1.802764 & 3.522230 & 4.224041 \\ \mathrm{H} & 0.207032 & 5.429713 & 3.821085 \\ \mathrm{H} & -0.984608 & 4.655762 & 4.854608 \\ \mathrm{H} & 1.630686 & 6.400576 & 7.695583\end{array}$

39

\begin{tabular}{|c|c|c|c|}
\hline $\mathrm{C}$ & 0.8482430979 & 0.4508569062 & -2.2104890935 \\
\hline $\mathrm{C}$ & -0.6336208523 & 0.0658721666 & -2.2655374535 \\
\hline $\mathrm{N}$ & -0.9061887208 & -0.3052555655 & -0.8721813545 \\
\hline $\mathrm{C}$ & 0.0598893090 & 0.1045837799 & -0.0231553466 \\
\hline $\mathrm{N}$ & 1.0846214515 & 0.6020346310 & -0.7659034591 \\
\hline $\mathrm{C}$ & -2.1999058515 & -0.8837121698 & -0.5491650966 \\
\hline $\mathrm{Ru}$ & -0.0421446282 & -0.0570289118 & 2.0000295787 \\
\hline $\mathrm{Cl}$ & 1.6414816347 & -1.7757410849 & 1.8892489490 \\
\hline $\mathrm{C}$ & 2.4693548737 & 0.7776653070 & -0.3465920615 \\
\hline $\mathrm{Cl}$ & -2.2388950332 & 0.9868866194 & 2.12 \\
\hline $\mathrm{C}$ & 0.8169108968 & 1.5594605471 & 2.176472 \\
\hline $\mathrm{C}$ & -0.8122997287 & -1.2205437818 & 4.065 \\
\hline $\mathrm{C}$ & 0.0847401891 & -0.3580126208 & 4.59 \\
\hline $\mathrm{H}$ & 1.0727761836 & 1.3799273051 & -2.74 \\
\hline $\mathrm{H}$ & 1.4995009098 & -0.340 & -2.60 \\
\hline $\mathrm{H}$ & -0.8385984633 & -0.7724183343 & -2.93967 \\
\hline $\mathrm{H}$ & -1.2752393970 & 0.90 & -2.5589 \\
\hline $\mathrm{H}$ & 2.8095218730 & 1.7977941291 & -0.56413 \\
\hline $\mathrm{H}$ & 2.5697923415 & 0.563 & 0.71 \\
\hline $\mathrm{H}$ & 3.1071219097 & 0.0719434539 & -0.8948512349 \\
\hline $\mathrm{H}$ & -2.3860947961 & -1.7203524084 & -1.2335288266 \\
\hline $\mathrm{H}$ & -2.2034896515 & -1.2500113975 & 0.4749852383 \\
\hline $\mathrm{H}$ & -3.0032033855 & -0.1452987149 & -0.6469198657 \\
\hline $\mathrm{C}$ & 1.4185903633 & 2.2105992146 & 3.3859363104 \\
\hline $\mathrm{H}$ & 0.8278469747 & 2.2131030525 & 1.292894 \\
\hline $\mathrm{H}$ & -0.2356887063 & 0.5970569919 & 4.9983250628 \\
\hline $\mathrm{H}$ & -1.8694648666 & -0.9769722891 & 4.0302247215 \\
\hline $\mathrm{H}$ & -0.5063794799 & -2.2166844418 & 3.7560372567 \\
\hline $\mathrm{H}$ & 1.1269208649 & -0.6377121119 & 4.7139209193 \\
\hline $\mathrm{C}$ & 2.4342864112 & 3.3159351674 & 3.0415560255 \\
\hline $\mathrm{H}$ & 0.5822934973 & 2.6581640336 & 3.9519434619 \\
\hline $\mathrm{H}$ & 1.8708393010 & 1.4645260250 & 4.0485154670 \\
\hline $\mathrm{C}$ & 2.9662228156 & 4.0207283104 & 4.3078152217 \\
\hline $\mathrm{H}$ & 3.2793092105 & 2.8925518151 & 2.4850819366 \\
\hline $\mathrm{H}$ & 1.9644811287 & 4.0616317905 & 2.3885464306 \\
\hline $\mathrm{C}$ & 3.9032801156 & 5.1044271882 & 4.0023699951 \\
\hline $\mathrm{H}$ & 2.1194566743 & 4.4230798680 & 4.8820186998 \\
\hline $\mathrm{H}$ & 3.4568855845 & 3.2843137265 & 4.9595524888 \\
\hline $\mathrm{C}$ & 4.6674627171 & 5.9966371933 & 3.7207846941 \\
\hline $\mathrm{H}$ & 5.3433208693 & 6.7863830920 & 3.483974004 \\
\hline
\end{tabular}




\begin{tabular}{|c|c|c|c|}
\hline $\mathrm{C}$ & 1.1116314337 & 0.5797167402 & -2.218612689 \\
\hline $\mathrm{C}$ & -0.3948830810 & 0.4558055092 & -2.4756235397 \\
\hline $\mathrm{N}$ & -0.9452194905 & 0.4501176032 & -1.110262042 \\
\hline $\mathrm{C}$ & 0.0090858330 & 0.2655115645 & -0.168289902 \\
\hline $\mathrm{N}$ & 1.2034946723 & 0.2417166573 & $-0.79221820 \varepsilon$ \\
\hline $\mathrm{C}$ & -2.3699801115 & 0.2131092075 & -0.957473543 \\
\hline $\mathrm{Ru}$ & -0.2339730039 & 0.0182476497 & 1.91557827 \\
\hline $\mathrm{Cl}$ & -1.1689506302 & -2.2078858261 & 1.49462100 \\
\hline $\mathrm{C}$ & 2.5143436896 & 0.1508249521 & -0.172652 \\
\hline $\mathrm{P}$ & -0.1115184877 & -0.6112419726 & 4.2565074 \\
\hline $\mathrm{C}$ & 0.3068993998 & 0.6820537943 & 5.50 \\
\hline $\mathrm{C}$ & -1.7832242968 & 0.9543873413 & 2.16 \\
\hline $\mathrm{Cl}$ & 1.2609213801 & 1.9383538739 & 2.33 \\
\hline $\mathrm{C}$ & -1.6090214747 & -1.4274692740 & 4.95 \\
\hline $\mathrm{C}$ & 1.2064184747 & -1.8815799422 & 4.49 \\
\hline $\mathrm{H}$ & 1.4822748127 & 1.6015417623 & $-2.3 \xi$ \\
\hline $\mathrm{H}$ & 1.7133789911 & -0.1065875994 & -2.82 \\
\hline $\mathrm{H}$ & -0.6564370084 & -0.4842851228 & -2.98 \\
\hline $\mathrm{H}$ & -0.8030768595 & 1.2863707139 & -3.0608692 \\
\hline $\mathrm{H}$ & 3.1270341544 & -0.5623315094 & -0.7377252 \\
\hline $\mathrm{H}$ & 3.0126900824 & 1.1267980531 & -0.15 \\
\hline $\mathrm{H}$ & 2.4170334726 & -0.1934154770 & 0.855432 \\
\hline $\mathrm{H}$ & -2.9429683870 & 1.0947313279 & -1.27 \\
\hline $\mathrm{H}$ & -2.6731797739 & -0.6419274229 & -1.5761689 \\
\hline $\mathrm{H}$ & -2.5884221426 & -0.0281120947 & \\
\hline$H$ & -2.6311487407 & 0.4373252469 & 2.6456802 \\
\hline $\mathrm{C}$ & -2.0525772821 & 2.3936333732 & 1.8 \\
\hline 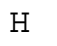 & -1.4192325101 & -1.8255972646 & 5.9602361 \\
\hline $\mathrm{H}$ & -2.4272161798 & -0.7017213713 & 5.01 \\
\hline $\mathrm{H}$ & -1.9072741715 & -2.2347755117 & 4.282234979 \\
\hline $\mathrm{H}$ & 1.2550056162 & -2.2170396190 & 5.540 \\
\hline $\mathrm{H}$ & 0.9905507648 & -2.7351580300 & 3.84 \\
\hline $\mathrm{H}$ & 2.1765262712 & -1.4593279879 & 4.21 \\
\hline 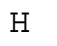 & 0.4860530894 & 0.2449120217 & 6.4911452 \\
\hline $\mathrm{H}$ & 1.1923265339 & 1.2251997784 & 5.16 \\
\hline $\mathrm{H}$ & -0.5169629676 & 1.3995844710 & 5.57115385 \\
\hline $\mathrm{C}$ & -2.2148463035 & 3.2269182129 & 3.1284988 \\
\hline 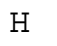 & -1.2456349279 & 2.8103695182 & 1.22840420 \\
\hline$=0$ & -2.9916616965 & 2.4510434710 & 1.25890218 \\
\hline 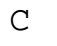 & -2.5438387303 & 4.7033604632 & 2.81791980 \\
\hline $\mathrm{H}$ & -3.0105185127 & 2.8051254923 & 3.75603377 \\
\hline 7 & -1.2808258049 & 3.1798120283 & 3.69688443 \\
\hline 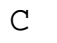 & -2.7271014948 & 5.5064386475 & 4.02923835 \\
\hline $\mathrm{H}$ & -1.7333502498 & 5.1329067790 & 2.21340628 \\
\hline $\mathrm{H}$ & -3.4539782477 & 4.7582017023 & 2.203489168 \\
\hline & -2.8755177686 & 6.1430257876 & 5.04527847 \\
\hline & -3.0031437156 & 6.7143064742 & 5.93590921 \\
\hline
\end{tabular}

41

C $\quad 0.8524286260$

C $\quad-0.6753317507$

$\mathrm{N} \quad-1.0596758611$

C $\quad-0.0236851513$

$\mathrm{N} \quad 1.1088242947$

C $\quad-2.4745679885$
0.0302165089

$-0.0322927896$

0.1910420727

0.0084358370

$-0.1505802267$

0.3171536837
$-2.0383419637$

$-2.1115483551$

$-0.7135911535$

0.1387906841

$-0.6021519193$

$-0.4014190170$ 


$\begin{array}{lr}\mathrm{Ru} & -0.1265015363 \\ \mathrm{Cl} & -2.1793096388 \\ \mathrm{C} & 2.4912529296 \\ \mathrm{Cl} & 1.1457807154 \\ \mathrm{C} & 1.0051725561 \\ \mathrm{C} & 1.4584418890 \\ \mathrm{C} & 1.0429894009 \\ \mathrm{C} & 1.5749697616 \\ \mathrm{C} & 1.2173351018 \\ \mathrm{C} & 0.9133488303 \\ \mathrm{H} & 1.2448777261 \\ \mathrm{H} & 1.3458879156 \\ \mathrm{H} & -1.0385786989 \\ \mathrm{H} & -1.1099856699 \\ \mathrm{H} & 2.9126818085 \\ \mathrm{H} & 2.5381990563 \\ \mathrm{H} & 3.0888973776 \\ \mathrm{H} & -2.9107048286 \\ \mathrm{H} & -2.9979748866 \\ \mathrm{H} & -2.6091190643 \\ \mathrm{H} & 1.3615680919 \\ \mathrm{H} & 2.6695064289 \\ \mathrm{H} & 1.0728148714 \\ \mathrm{H} & 2.5576365590 \\ \mathrm{H} & -0.0513025075 \\ \mathrm{H} & 1.4237070877 \\ \mathrm{H} & 0.6469956341 \\ \mathrm{H} & 1.1863942732\end{array}$

42

C

$\mathrm{N}$

C

C

$\mathrm{N}$

C

C

$\mathrm{Ru}$

$\mathrm{Cl}$

$\mathrm{Cl}$

C

C

C

$\mathrm{H}$

$\mathrm{H}$

$\mathrm{H}$

$\mathrm{H}$

$\mathrm{H}$

$\mathrm{H}$

$\mathrm{H}$

$\mathrm{H}$

$\mathrm{H}$

$\mathrm{H}$

C

$\mathrm{H}$

$\mathrm{H}$

C
-0.0275198033
-1.1493023931
0.0034095652
1.9578288051
-1.4494519901
-1.9956859222
-1.1955842364
-1.8418181108
-1.0858828940
-0.4452517083
1.0051221379
-0.7509351711
-1.0149472493
0.7336687936
0.9215548422
0.0851291930
-0.8502027234
1.0659099465
-0.6370605692
0.6326443703
-2.0049297418
-1.9310562723
-3.0287338419
-2.0965667046
-1.1364260179
-0.1699430287
0.1181393658
-2.8667598367

2.1074099557

2. 5330490412

$-0.1649503068$

2.3336339005

2. 2672191446

3. 5880391456

4.8317670994

6.1297434103

7.3316722864

8.3095063485

$-2.3620124069$

$-2.6256030983$

$-2.4459778564$

$-2.7615283301$

$-0.5946520766$

0.9171017128

$-0.5077230094$

$-1.0724338563$

$-0.5360349720$

0.6300694279

1.3899179823

6.0702595424

3. 6573748442

3. 5533686549

4.8864902730

4. 7570327409

9.1742321864

6.2142593383
0.0331676037

$-0.2284125002$

$-0.0433619232$

0.0484229452

0.3064243691

$-0.2080356309$

0.5938374722

0.0497877236

$-1.6457588361$

2.1816989652

$-1.2483753793$

0.3200142863

0.2688690714

0.8817251548

$-0.8792004685$

$-0.8922920717$

0.8562701571

0.5491357987

0.0682894638

$-1.1819342142$

1.3932343884

$-0.2929221110$

0.9300312168

$-1.4007723634$

$-2.0812605115$

0.3116187812

0.1621678567
0.1631382670 $-0.5495825711$

$-1.9948675656$

$-2.1020638810$

$-0.7106077693$

$-0.0527798501$

$-0.4062699543$

2.1862682474

1. 9474186829

2. 2234660195

2. 4707013554

4.2757586105

4. 7219082525

$-2.3214101934$

$-2.5623759378$

$-2.4477359829$

$-2.7582660376$

$-0.6023284187$

0.9993256315

$-0.1928579182$

$-1.0730990602$

$-0.5325897570$

0.6253282598

3. 5958452612

1. 7539183132

4.0996939878

5.4179219749 


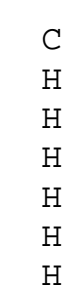

C

$\mathrm{N}$

$$
\begin{array}{r}
-2.4117625617 \\
-2.2305032260 \\
-0.9428455344 \\
-0.1828564922 \\
-0.9771840337 \\
-3.3552677054 \\
-0.3844769298 \\
1.3407282950 \\
1.2614243325 \\
-1.1395277786 \\
-0.9004584545 \\
0.3368646865 \\
0.0741276513 \\
-0.8002635732 \\
-0.7046233348 \\
-2.8006778859 \\
-3.1127067730 \\
1.5570934361 \\
1.6849724262 \\
1.6530186881 \\
-4.2117527333 \\
-3.6137229452 \\
-3.1027502313 \\
2.6065492560 \\
1.4447318112 \\
-1.8884861607 \\
1.6137802628 \\
3.4593000297 \\
2.7525465332 \\
1.4035708984 \\
2.0961063483 \\
2.5175489758 \\
3.5101679608 \\
2.0595031270
\end{array}
$$

$$
\begin{array}{r}
-1.4112889720 \\
0.0463679436 \\
0.3807713883 \\
-0.7245366002 \\
-1.8891528737 \\
0.9291065448 \\
2.3172601662 \\
2.6770977658 \\
-0.7533151934 \\
1.9441543039 \\
4.3578859899 \\
4.5392962645 \\
2.5537091879 \\
-2.0932308601 \\
-2.7788285848 \\
-1.7816870628 \\
-1.6877365260 \\
-0.7543932053 \\
0.1233935948 \\
-1.6519101452 \\
0.6013549031 \\
0.9401999732 \\
1.9465609869 \\
3.0263785999 \\
2.5900405110 \\
4.7717261629 \\
5.2829981109 \\
2.9142347323 \\
2.3541269727 \\
6.2869378604 \\
5.3917381469 \\
4.4877514361 \\
4.9457781088 \\
4.4801138801
\end{array}
$$

44

$\begin{array}{lr}\mathrm{N} & 1.1852455682 \\ \mathrm{C} & 0.9511853581 \\ \mathrm{C} & -0.4512442998 \\ \mathrm{~N} & -0.9587575226 \\ \mathrm{C} & 0.0390333153 \\ \mathrm{C} & -2.2780775333 \\ \mathrm{Ru} & -0.1462741877 \\ \mathrm{C} & 0.8075016845 \\ \mathrm{C} & 1.0408871699 \\ \mathrm{C} & 0.2467956990\end{array}$

0.2750009481

0.3023139903

$-0.3032344457$

$-0.1589872315$

0.0850107049

$-0.6989580429$

0.1072099633

1. 6652528338

2. 2208607794

2. 0854686108
$-0.7826331062$

$-2.2312225430$

$-2.3403747221$

$-0.9703771535$

$-0.0805812979$

$-0.6770468349$

1. 8820076231

2.1262678655

3. 4393765810

4. 5317119628 


$\begin{array}{lr}\mathrm{C} & 2.5339840727 \\ \mathrm{Cl} & 1.3376967202 \\ \mathrm{Cl} & -2.3203389561 \\ \mathrm{H} & 0.9903818612 \\ \mathrm{H} & 1.7152551474 \\ \mathrm{H} & -0.4250002264 \\ \mathrm{H} & -1.0964470389 \\ \mathrm{H} & 3.2258393817 \\ \mathrm{H} & 2.8371464807 \\ \mathrm{H} & 2.5960712645 \\ \mathrm{H} & -2.9959679781 \\ \mathrm{H} & -2.2785707499 \\ \mathrm{H} & -2.5856934818 \\ \mathrm{H} & 1.2622745184 \\ \mathrm{C} & 2.2787483473 \\ \mathrm{C} & 0.8138233229 \\ \mathrm{H} & -0.7013272439 \\ \mathrm{C} & 1.9219014048 \\ \mathrm{H} & 0.0571753888 \\ \mathrm{H} & 1.2276196323 \\ \mathrm{H} & 2.5062469281 \\ \mathrm{H} & 3.1588714595 \\ \mathrm{H} & 1.5148356930 \\ \mathrm{H} & 2.7893393941\end{array}$

45

C

C

C

C

C

C

$\mathrm{Ru}$

Cl

$\mathrm{N}$

C

C

$\mathrm{N}$

C

C

Cl

$\mathrm{H}$

$\mathrm{H}$

$\mathrm{H}$

$\mathrm{H}$

$\mathrm{H}$

$\mathrm{H}$

$\mathrm{H}$

$\mathrm{H}$

$\mathrm{H}$

$\mathrm{H}$

$\mathrm{H}$

$\mathrm{H}$

$\mathrm{H}$

$\mathrm{H}$
0.5067947239

$-1.7220561419$

1.0001697214

1. 3360949479

$-0.2807234948$

$-1.3664317363$

0.2251354150

$-0.1300659156$

1. 5536698727

0.2353872633

$-0.2869984610$

$-1.7949067314$

$-0.4068137146$

2. 2028301135

3. 0510636809

2. 7789388607

1. 5566628201

3. 6877245323

3. 3384501689

2. 0328834011

3. 7993468327

2. 3964595527

4. 6925878384

3. 7872403221
$-0.2931781355$

2. 1458151807

2. 2687242835

$-2.6042584896$

$-2.7552428100$

$-2.6219205730$

$-3.0491999684$

$-0.8565302836$

$-0.4350912313$

0.7580824887

$-1.3944703740$

$-0.7692931569$

0.3246875386

1. 2871127865

3. 7798095726

5.7408241986

4.5357549380

5.1464348591

6.3045266979

6.4387759509

3. 0103474003

3. 8547969991

4. 9849718071

5.8062694748
2.1972162839
3.6086940705
5.0212945792
2. 8721826270
3.6739187472
2.4049418621
2.2590136087
3.4033928049
0.2588252475
2. 2973983581
4.5070507658
2. 9283220758
5.6799155141
0.6635717755
1.6581460687
2.1182342277
0.0094879736
1.6649548510
$-1.3685631474$
$-0.3476323273$
0.0993006461
2.6901085860
0.7233927936
1.1621970587
$-0.2189394983$
0.9400074855
$-1.0518544124$
0.2022408448
0.0065666658
$-0.5784713732$
$-2.3443202971$
$-2.3438406247$
$-0.9064362128$
$-0.9183739140$
0.1361964233
1.4696521234
2.4567869552
$-0.7920229108$
$-2.3109480172$
$-0.5388859492$
0.2810017345
1.0803024615
1.4837619435
$-2.3829192058$
$-0.7530004852$
1.2946484474
$-3.1651743038$
0.8333187591
$-1.0979383654$
$-0.9711029381$
$-0.8893841953$
$-2.9213124258$
2.0634429454
2. 7439320084
$-2.7246251916$
0.7104581128
3. 2327655769
$-1.6673118316$
2.1326510778
2. 3854425520
$-0.6211423442$
0.0674169871
$-0.8328089384$
$-2.7517496472$
$-0.9562901156$
$-2.8564126265$
0.0399527784
$-2.4011688337$
$-0.9351655442$
0.5446641427
$-0.0574000835$
2.1886144890
1.4842818792
$-0.7362937968$
1.8674902434
0.3374633914
3.6358472952
4.5760933801
6.2315767824
2.1531788639
6.4027523287
1.2688671544
2.7295766805
3.5449198606
2. 8710613951 
46

$\begin{array}{lrrr}\mathrm{N} & 1.2176123176 & 0.2622296122 & -0.7295567735 \\ \mathrm{C} & 1.0650209045 & 0.1578907194 & -2.1854600742 \\ \mathrm{C} & -0.2699481612 & -0.5813958192 & -2.3132358724 \\ \mathrm{~N} & -0.8722460679 & -0.3607875553 & -0.9926407845 \\ \mathrm{C} & 0.0498599085 & 0.0349262346 & -0.0755922896 \\ \mathrm{C} & -2.1500747580 & -0.9998374341 & -0.7135830119 \\ \mathrm{Ru} & -0.2604581923 & 0.1854250363 & 1.8605475544 \\ \mathrm{C} & 0.6324574706 & 1.7900231083 & 2.0355592321 \\ \mathrm{C} & 0.9151882285 & 2.3512149385 & 3.3275398829 \\ \mathrm{C} & 1.5625067859 & 3.5257280092 & 3.5357809741 \\ \mathrm{C} & 2.5119219526 & 0.6410807824 & -0.1878446364 \\ \mathrm{Cl} & 1.2976060952 & -1.5359931862 & 2.3443803304 \\ \mathrm{Cl} & -2.4940124377 & 0.9953845899 & 2.0350891667 \\ \mathrm{H} & 1.0321314033 & 1.1595519279 & -2.6385117906 \\ \mathrm{H} & 1.9060255011 & -0.3895749206 & -2.6226466307 \\ \mathrm{H} & -0.1323148271 & -1.6588029383 & -2.4894237874 \\ \mathrm{H} & -0.9123867631 & -0.1813481730 & -3.1036760129 \\ \mathrm{H} & 3.2854642119 & 0.0344736379 & -0.6728054688 \\ \mathrm{H} & 2.7338211382 & 1.7002008173 & -0.3798320264 \\ \mathrm{H} & 2.5357233485 & 0.4416524882 & 0.8812165223 \\ \mathrm{H} & -2.8518741481 & -0.7490983625 & -1.5159282017 \\ \mathrm{H} & -2.0388937758 & -2.0933586778 & -0.6721902639 \\ \mathrm{H} & -2.5535790735 & -0.6276326269 & 0.2262148160 \\ \mathrm{H} & 0.9354532651 & 2.3890326409 & 1.1699641383 \\ \mathrm{C} & 0.5771975944 & 1.6829889872 & 4.6545863447 \\ \mathrm{C} & 1.7514021861 & 3.8261218332 & 4.9975383691 \\ \mathrm{H} & 1.9382645909 & 4.1669439126 & 2.7422451317 \\ \mathrm{C} & 0.8070006828 & 2.8103780987 & 5.6949813413 \\ \mathrm{H} & -0.4507325688 & 1.3001687133 & 4.6785564861 \\ \mathrm{H} & 1.2441909957 & 0.8254139390 & 4.8175122481 \\ \mathrm{H} & 2.8051106693 & 3.6685755450 & 5.2802402162 \\ \mathrm{H} & 1.5212331521 & 4.8692533184 & 5.2504918179 \\ \mathrm{H} & -0.1473022770 & 3.2999508650 & 5.9184852490 \\ \mathrm{H} & 1.2127691181 & 2.4361828711 & 6.6395397984\end{array}$




$\begin{array}{lr}\mathrm{Cl} & -1.8857880971 \\ \mathrm{C} & -0.8919267047 \\ \mathrm{C} & -1.7439934936 \\ \mathrm{H} & 0.6356702863 \\ \mathrm{H} & -0.4572757111 \\ \mathrm{H} & 1.0225096656 \\ \mathrm{H} & 0.0223811325 \\ \mathrm{H} & -1.5228867785 \\ \mathrm{H} & 0.9322337693 \\ \mathrm{H} & 1.8774075667 \\ \mathrm{H} & 2.3682006988 \\ \mathrm{H} & -2.4120306898 \\ \mathrm{H} & -0.9188909828 \\ \mathrm{H} & -1.9212585672 \\ \mathrm{H} & -1.2559496902 \\ \mathrm{H} & -0.0524852054 \\ \mathrm{H} & -1.6820067201 \\ \mathrm{H} & -1.9840845836 \\ \mathrm{H} & -2.6207442203 \\ \mathrm{H} & -1.4791506127 \\ \mathrm{H} & 0.6183653715 \\ \mathrm{H} & 1.3842379122 \\ \mathrm{H} & 1.8056486513 \\ \mathrm{C} & 3.6510168167 \\ \mathrm{C} & 4.6297768740 \\ \mathrm{H} & 2.5925469138 \\ \mathrm{H} & 5.1659403941 \\ \mathrm{H} & 4.9671987954 \\ \mathrm{C} & 4.8650203576 \\ \mathrm{H} & 3.3255909792 \\ \mathrm{H} & 3.8685947327 \\ \mathrm{H} & 4.8702923451 \\ \mathrm{H} & 5.8237092968\end{array}$

48

C

C

C

C

C

C

$\mathrm{Ru}$

C

C

C

$\mathrm{N}$

C

C

$\mathrm{N}$

C

C

$\mathrm{Cl}$

$\mathrm{Cl}$

$\mathrm{H}$

$\mathrm{H}$
1.6042140149

1. 5051473561

$-1.1941735935$

2. 6143465824

0.5428697350

$-0.4295445167$

$-2.3597691159$

$-1.5840315649$

2. 3958718781

1. 7273266190

1. 3862713564

$-2.4270861227$

$-2.8784604678$

$-1.4922633500$

1. 2924879337

2. 2056411608

1. 9662060238

$-1.2924978035$

$-0.8162038677$

$-2.1783338915$

$-0.9079007251$

$-1.6099956906$

0.0300051830

0.7530240657

3.0139254886

3. 9856986770

3. 2591152023

3. 7456624304

1. 5431434886

$-0.0568893120$

0.2751631856

1. 4886825338

1. 1467289471
1.6140301163

5.0404218031

4. 4479557748

1.9788903900

$-3.0474527322$

$-3.2499801260$

$-2.2072121091$

$-2.6447985472$

$-1.6491101423$

$-0.2953253333$

$-1.9707656310$

$-0.3874212744$

0.4999109710

1.0289338678

6.0519655172

5.0997767262

4. 4410772660

5.5129217982

3. 9129620747

4. 0484981866

6.2743199500

4.8076967554

5.3286837817

2. 5140972842

2. 6325190839

2. 2581077152

1. 7007850108

3.3780951249

3. 0654414322

3.1693468024

1. 5500242036

4.1610351890

2. 7170531149
$-0.3189060000$

$-0.6236320000$

0.7039340000

1.7051410000

1.2170810000

0.9991910000

$-0.0443130000$

$-0.1488740000$

$-0.6503850000$

0.0422500000

$-0.5687920000$

$-0.6172160000$

0.4883960000

0.5909760000

$-1.4301560000$

1.6093650000

$-2.3407110000$

1.9776360000

1.4465270000

0.2398930000

$-1.6035010000$

$-0.4271420000$
3.8552950000

3.0351900000

3.0310720000

3.4343490000

3.7507910000

2.6051670000

1.9196410000

4.3885060000

3.7706480000

$-0.1003360000$

$-0.8690480000$

$-2.2802010000$

$-2.3265360000$

$-0.9110140000$

$-0.3498610000$

$-0.5401670000$

1.8816870000

1.8136220000

$-2.6660100000$

$-2.9556500000$

$-2.5229070000$

$-2.9377410000$ 


$\begin{array}{lr}\mathrm{H} & 1.5071750000 \\ \mathrm{H} & 2.2697940000 \\ \mathrm{H} & 2.8050770000 \\ \mathrm{H} & -2.7545510000 \\ \mathrm{H} & -1.5681650000 \\ \mathrm{H} & -2.3300020000 \\ \mathrm{H} & 0.9305110000 \\ \mathrm{H} & 3.0818990000 \\ \mathrm{H} & -0.3531780000 \\ \mathrm{H} & -1.5627940000 \\ \mathrm{H} & -2.3662320000 \\ \mathrm{H} & 0.4489370000 \\ \mathrm{H} & 5.4426260000 \\ \mathrm{H} & 5.1714620000 \\ \mathrm{H} & 2.6896100000 \\ \mathrm{H} & 3.5099820000 \\ \mathrm{H} & 4.4001100000 \\ \mathrm{H} & 5.4370230000\end{array}$

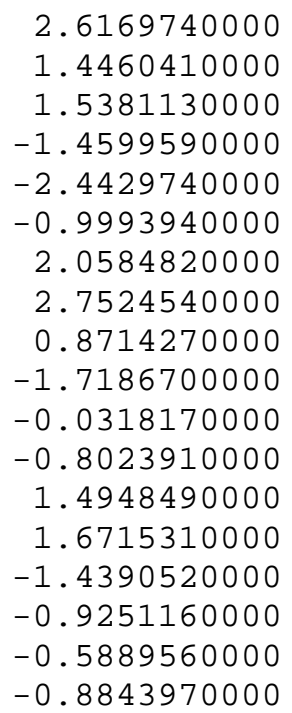

2.6169740000

$-0.6208020000$

0.4830660000

$-1.2060350000$

$-1.0734730000$

$-0.1482210000$

0.5759250000

2.7342200000

3.4815150000

4.7588940000

3. 5869710000

3. 6449150000

4. 6753810000

2.9334470000

4.6599330000

3. 4489270000

2. 0038770000

4.9047280000

3. 5103660000

49

C

C

C

C

C

C

$\mathrm{Ru}$

C

C

C

$\mathrm{N}$

C

$\mathrm{N}$

C

$\mathrm{Cl}$

$\mathrm{Cl}$

$\mathrm{H}$

$\mathrm{H}$

$\mathrm{H}$

$\mathrm{H}$

$\mathrm{H}$
4.8483656483

3. 3945992981

2. 5921430728

3. 4192363725

4.8688425116

1.1514947146

$-0.2808115076$

0.3179791028

$-0.8623708356$

$-0.7343521277$

$-1.7593031509$

$-1.7947568974$

$-0.9171341585$

$-0.1442428884$

$-2.4824744056$

0.7922276682

0.7502328370

$-1.7575130021$

$-1.5159890377$

$-0.2536404846$

$-1.3647747454$

$-2.8202072361$

0.2675473960

1.3850046265

1.4635293664

$-3.4917391680$

$-1.9705081936$

$-2.5734659887$

0.8555671379

3.0777392537

0.2144548743

$-0.8089670058$

$-1.8246464318$

1. 2279978488

5.2384710714

5.5119659853

3. 0433127403
0.0513967087

$-0.4460298422$

0.8409713373

1. 9031234414

1. 5073742081

1.0241587242

$-0.1598163832$

0.3820571363

$-0.3904234130$

$-0.5309116048$

$-1.3283667687$

$-1.5815394653$

$-0.4408883054$

$-0.0733344385$

$-2.1797221747$

1.0361079396

$-2.3849029690$

1. 8009346512

0.4176789878

$-0.7459295174$

$-2.5679253509$

$-1.5505244129$

1. 9952512953

1.0707980685

0.8820340064

$-2.3513033540$

$-3.1342180264$

$-1.6687681290$

2. 0794248703

2. 9274359558

1. 4312562178

$-1.4566427807$

0.1033356099

$-0.1148819647$

1. 5609442993

2. 1764820912

$-1.0957053266$
2.9407860403

2. 7322860646

2.6265043164

2. 4575988174

2.4056070842

2.5984085501

2.2676921613

4. 4783824288

4.3367122668

0.2955206936

$-0.0494167871$

$-1.4967695647$

$-2.0244206870$

$-0.8249239855$

0.8800726493

$-0.8976594204$

2. 3563040447

2.1389732528

$-2.3598672263$

$-2.8385216975$

$-1.7197936291$

$-1.8764821544$

$-1.0199033762$

0.0142534154

$-1.7506337420$

0.4933189986

1. 0327806365

1. 8405085066

2.6176860656

2.3302483196

4. 7405772815

4.5391566794

4.4455438394

4.7977036897

1.3688530921

2. 9916035405

3. 5380573432 


$\begin{array}{rrrr}\mathrm{H} & 3.2777190072 & -1.0361165596 & 1.8162082939 \\ \mathrm{H} & 5.0851699538 & 0.0574634354 & 4.0112307198 \\ \mathrm{H} & 5.5866369604 & -0.5897691425 & 2.4500556831\end{array}$

50

\begin{tabular}{|c|c|c|c|}
\hline $\mathrm{N}$ & -1.0567358583 & -0.8527432831 & -0.541012593 \\
\hline $\mathrm{C}$ & -0.1080299586 & -0.1629254566 & 0.114431807 \\
\hline $\mathrm{N}$ & 0.6845975199 & 0.4674931996 & -0.76274486 \\
\hline $\mathrm{C}$ & 0.3680788578 & 0.0676108413 & -2.1432825 \\
\hline $\mathrm{C}$ & -1.0355493133 & -0.5353564882 & -1.97860993 \\
\hline $\mathrm{Ru}$ & 0.0869334094 & -0.1206799971 & 2.1255777 \\
\hline $\mathrm{Cl}$ & -1.5961188591 & 1.6723899716 & 2.0418224 \\
\hline $\mathrm{C}$ & 1.9599790199 & 1.0871463751 & -0.4345223 \\
\hline $\mathrm{C}$ & -2.2532828064 & -1.4015981500 & 0.0811230 \\
\hline $\mathrm{C}$ & 1.1429166822 & 0.9428446676 & 3.4166742 \\
\hline $\mathrm{C}$ & 0.3011850137 & 0.0310761770 & 4.3965816 \\
\hline $\mathrm{C}$ & -0.9085537164 & -0.7772365406 & 3.7602463 \\
\hline $\mathrm{C}$ & -1.0679843873 & -2.2089541621 & 4.042363 \\
\hline $\mathrm{C}$ & -2.2750356391 & -2.8166978629 & 3.984552 \\
\hline $\mathrm{Cl}$ & 1.8983490870 & -1.7365044510 & 1.915569 \\
\hline $\mathrm{H}$ & 0.3923774628 & 0.9304922152 & -2.815128 \\
\hline $\mathrm{H}$ & 1.0979233274 & -0.6761695932 & -2.4937778 \\
\hline $\mathrm{H}$ & -1.1936436135 & -1.4350588640 & -2.5802568 \\
\hline $\mathrm{H}$ & -1.8260212584 & 0.1923838100 & -2.21177951 \\
\hline $\mathrm{H}$ & 1.9080948173 & 1.4940105368 & 0.5765141 \\
\hline $\mathrm{H}$ & 2.7793574416 & 0.3599114490 & -0.481800446 \\
\hline $\mathrm{H}$ & 2.1479894816 & 1.9067736665 & -1.1353471 \\
\hline $\mathrm{H}$ & -2.6146793515 & -2.2385302453 & -0.5248171 \\
\hline $\mathrm{H}$ & -2.0092094083 & -1.7777878935 & 1.0767691 \\
\hline $\mathrm{H}$ & -3.0377518442 & -0.6403554400 & 0.1724587 \\
\hline $\mathrm{H}$ & 2.2089874504 & 0.7170001556 & 3.4415849 \\
\hline $\mathrm{H}$ & 0.8875444695 & 1.9998555814 & 3.4812930 \\
\hline $\mathrm{H}$ & -0.1816882491 & 0.7223611161 & 5.0915953 \\
\hline $\mathrm{H}$ & -1.8435668543 & -0.2246789203 & 3.8509483 \\
\hline $\mathrm{H}$ & 0.9722914454 & -0.6686315266 & 4.8920471 \\
\hline $\mathrm{C}$ & 0.0160182968 & -3.2185073576 & 4.390 \\
\hline $\mathrm{C}$ & -2.1962592064 & -4.2953332912 & 4.26115513 \\
\hline $\mathrm{H}$ & -3.2029320742 & -2.3078741913 & 3.7328553 \\
\hline $\mathrm{H}$ & -2.9968062445 & -4.6525632260 & 4.9215245 \\
\hline $\mathrm{H}$ & -2.2917742604 & -4.8675358182 & 3.3238448 \\
\hline 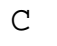 & -0.7843862812 & -4.4502417852 & 4.88392900 \\
\hline $\mathrm{H}$ & 0.6220817920 & -3.4358384932 & 3.5031384 \\
\hline & 0.7145561270 & -2.8492515860 & 5.15040853 \\
\hline & -0.3048774354 & -5.3973410569 & 4.617 \\
\hline & -0.8698727105 & -4.4222319906 & 5.9768385 \\
\hline
\end{tabular}

51

$\begin{array}{lrrr}\text { C } & -1.2748159317 & -4.4702479192 & 5.0307497522 \\ \text { C } & -0.2634534318 & -3.3105417918 & 4.8351578589 \\ \text { C } & -1.1431863390 & -2.1448379695 & 4.4129282638 \\ \text { C } & -2.3834744031 & -2.5720191836 & 4.0851780639 \\ \text { C } & -2.5290590612 & -4.0665693008 & 4.2104710108 \\ \text { C } & -0.7576817281 & -0.7301365006 & 4.3874627243 \\ \text { C } & 0.5181274586 & -0.2288026028 & 4.7013099759 \\ \text { Ru } & 0.1463477746 & -0.4012980387 & 2.3512302238\end{array}$




$\begin{array}{lr}\mathrm{Cl} & 1.8125827251 \\ \mathrm{C} & -0.0097569798 \\ \mathrm{~N} & -1.0755208401 \\ \mathrm{C} & -0.9998633916 \\ \mathrm{C} & 0.4873634187 \\ \mathrm{~N} & 0.8830757607 \\ \mathrm{C} & -2.3612060693 \\ \mathrm{C} & 2.2723346530 \\ \mathrm{Cl} & -1.5144813700 \\ \mathrm{C} & 1.4405225470 \\ \mathrm{H} & 0.6736149322 \\ \mathrm{H} & 1.0630553005 \\ \mathrm{H} & -1.3259179420 \\ \mathrm{H} & -1.6430974176 \\ \mathrm{H} & 2.3456038471 \\ \mathrm{H} & 2.9054014166 \\ \mathrm{H} & 2.6154390592 \\ \mathrm{H} & -2.8416509296 \\ \mathrm{H} & -2.2138327027 \\ \mathrm{H} & -3.0095429494 \\ \mathrm{H} & 2.4794731628 \\ \mathrm{H} & 1.2045473068 \\ \mathrm{H} & 0.5858136173 \\ \mathrm{H} & -1.5830533638 \\ \mathrm{H} & 1.3375362621 \\ \mathrm{H} & -3.1857777759 \\ \mathrm{H} & 0.4765775329 \\ \mathrm{H} & 0.2986452059 \\ \mathrm{H} & -3.4695599093 \\ \mathrm{H} & -2.5300171712 \\ \mathrm{H} & -0.8652360713 \\ \mathrm{H} & -1.5479577547 \\ & \end{array}$

52

$\begin{array}{lr}\mathrm{N} & -0.9998932080 \\ \mathrm{C} & -0.0523346445 \\ \mathrm{~N} & 0.7656128480 \\ \mathrm{C} & 0.4697306809 \\ \mathrm{C} & -0.9372485704 \\ \mathrm{Ru} & 0.0720662554 \\ \mathrm{Cl} & -2.0338689841 \\ \mathrm{C} & 2.0767422176 \\ \mathrm{C} & -2.2186204354 \\ \mathrm{C} & 0.9935835919 \\ \mathrm{C} & 0.5012943172 \\ \mathrm{C} & -0.7063808922 \\ \mathrm{C} & -0.8995011676 \\ \mathrm{C} & -2.0645583531 \\ \mathrm{Cl} & 2.1199155541 \\ \mathrm{H} & 0.5196325860 \\ \mathrm{H} & 1.1992721609 \\ \mathrm{H} & -1.0904530997 \\ \mathrm{H} & -1.7179359038 \\ \mathrm{H} & 2.0120194675 \\ \mathrm{H} & 2.8094749640 \\ \mathrm{H} & 2.3929507982 \\ \mathrm{H} & -2.4747564938\end{array}$

$$
\begin{array}{r}
-2.2062902324 \\
-0.5888745452 \\
-1.1535013602 \\
-1.0296926197 \\
-0.7244444626 \\
-0.2332688521 \\
-1.4161073642 \\
0.1288187646 \\
1.4128719928 \\
0.7991952021 \\
0.0325643836 \\
-1.6277475962 \\
-1.9524827360 \\
-0.2045095142 \\
0.7055718142 \\
-0.7616664879 \\
0.7463987047 \\
-2.2511901233 \\
-1.7018922710 \\
-0.5328325452 \\
0.4899217879 \\
1.8560702629 \\
0.7787867347 \\
-0.0228059598 \\
-0.9051769086 \\
-1.9195569759 \\
-3.5259838303 \\
-3.0898841594 \\
-4.3663004537 \\
-4.5354735408 \\
-5.4379679049 \\
-4.5496839159
\end{array}
$$

2. 2692108862

0.3071054318

$-0.2960675547$

$-1.7596991949$

$-1.9656260522$

$-0.6348101080$

0.3328103987

$-0.4043201646$

2.1972601261

2.8742091800

$-2.7331805990$

$-2.2144986875$

$-2.2490560855$

$-2.0987132785$

0.5164723005

$-0.3129456819$

$-1.2406889469$

$-0.1884045663$

1. 3752818198

0.3023040174

3. 0315195552

3. 0373513741

5.1004113390

4.3981789928

4. 9212112972

3. 7485848204

4.0561302529

5.7514964358

4. 6904484257

3. 2131695974

4. 7251921063

6.0895228087

$\begin{array}{rr}-0.8333169611 & -0.5206328166 \\ -0.1236626311 & 0.1285384330 \\ 0.4344449633 & -0.7872109587 \\ -0.0512476773 & -2.1459562350 \\ -0.6276943021 & -1.9748993889 \\ -0.0584909184 & 2.1827254246 \\ 1.2277350365 & 2.2037798761 \\ 1.0132614383 & -0.5314486778 \\ -1.3831205691 & 0.0516478084 \\ 1.4944439059 & 2.4090366881 \\ -0.2303764739 & 4.6263684031 \\ -0.8076808237 & 4.3195104276 \\ -2.2250363363 & 4.0031753084 \\ -2.7308861184 & 3.5400141645 \\ -1.4126263606 & 1.9748704698 \\ 0.7652058325 & -2.8723450101 \\ -0.8225429337 & -2.4321810041 \\ -1.5693238879 & -2.5108231871 \\ 0.0832113179 & -2.2831502935 \\ 1.7287140677 & 0.2895468377 \\ 0.2431444121 & -0.2690700816 \\ 1.5490613498 & -1.4313018631 \\ -2.3008985610 & -0.4900104572\end{array}$ 


$\begin{array}{rr}\mathrm{H} & -2.0618511190 \\ \mathrm{H} & -3.0466485867 \\ \mathrm{H} & 2.0704664401 \\ \mathrm{H} & 0.4884928886 \\ \mathrm{H} & 0.5303579569 \\ \mathrm{H} & -1.6100281753 \\ \mathrm{H} & 1.4268371576 \\ \mathrm{C} & 0.1415495517 \\ \mathrm{C} & -1.9934502825 \\ \mathrm{H} & -2.9500621557 \\ \mathrm{C} & -0.6859030043 \\ \mathrm{H} & 0.9169884720 \\ \mathrm{H} & 0.6519590182 \\ \mathrm{H} & -2.8779805505 \\ \mathrm{H} & -1.9407062265 \\ \mathrm{H} & -0.1393745971 \\ \mathrm{H} & -0.9352813079\end{array}$

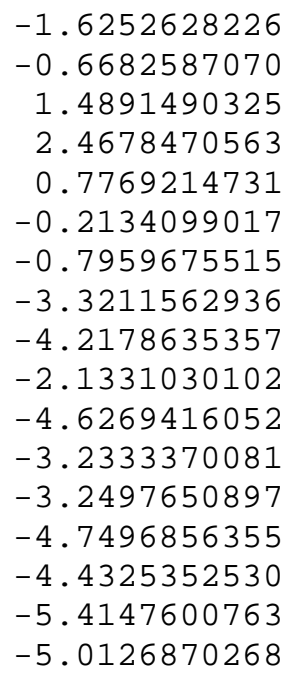

1.1012448146 $-0.0157068040$

2. 6195441529

2. 4091553476

5.0283246374

4.4220036965

4. 6238444794

4.1729076405

3. 3068228084

3. 3387129308

4. 0382021217

3. 4031884874

5.1415800202

3. 6801787905

2. 2272843470

3. 5107961340

5.0334407433

\section{8-Me}

\begin{tabular}{|c|c|}
\hline $\mathrm{N}$ & -0.2240179620 \\
\hline $\mathrm{C}$ & 0.1791389438 \\
\hline $\mathrm{N}$ & 0.8214667445 \\
\hline C & 0.763216368 \\
\hline $\mathrm{C}$ & 0.245870199 \\
\hline $\mathrm{Ru}$ & -0.2530950699 \\
\hline $\mathrm{Cl}$ & -1.6552491737 \\
\hline $\mathrm{C}$ & 1.165361376 \\
\hline $\mathrm{C}$ & -0.9669735708 \\
\hline $\mathrm{C}$ & 1.385715239 \\
\hline $\mathrm{C}$ & 2.743194712 \\
\hline $\mathrm{C}$ & 3.794667992 \\
\hline $\mathrm{C}$ & 5.122701230 \\
\hline $\mathrm{C}$ & 4.747424846 \\
\hline $\mathrm{C}$ & 3.249015624 \\
\hline $\mathrm{Cl}$ & 0.485411092 \\
\hline $\mathrm{C}$ & -0.894166104 \\
\hline $\mathrm{C}$ & -1.560077115 \\
\hline $\mathrm{C}$ & -2.970442781 \\
\hline $\mathrm{H}$ & 0.073950961 \\
\hline $\mathrm{H}$ & 1.750343490 \\
\hline $\mathrm{H}$ & 1.036994200 \\
\hline $\mathrm{H}$ & -0.572194713 \\
\hline $\mathrm{H}$ & 0.532861148 \\
\hline $\mathrm{H}$ & 0.992997944 \\
\hline $\mathrm{H}$ & 2.217305854 \\
\hline $\mathrm{H}$ & -1.847169317 \\
\hline $\mathrm{H}$ & -0.344452146 \\
\hline $\mathrm{H}$ & -1.274683394 \\
\hline $\mathrm{H}$ & 1.334343547 \\
\hline $\mathrm{H}$ & 3.694325205 \\
\hline $\mathrm{H}$ & -1.335851048 \\
\hline $\mathrm{H}$ & -1.099003712 \\
\hline $\mathrm{H}$ & 0.051854161 \\
\hline $\mathrm{H}$ & 5.554367897 \\
\hline $\mathrm{H}$ & 5.862664069 \\
\hline $\mathrm{H}$ & 2.693985215 \\
\hline $\mathrm{H}$ & 3.125352076 \\
\hline
\end{tabular}

-1.1545927058
-0.1102764264
0.7639688987
0.3632722484
-1.0760995966
0.1415280826
1.9879104025
2.1502273890
-2.3354952363
0.9152922758
0.5760960341
1.2743473297
0.7333806472
-0.6582619035
-0.5490990617
-2.1245614539
0.9574067000
-0.2146865294
-0.3423390295
1.0252692042
0.4393603640
-1.8202867692
-1.2837437892
2.8129404839
2.3815072073
2.3309218047
-2.4435963316
-3.2340169452
-2.2499590799
1.7675596447
2.1412016117
1.9116785169
-1.1274561089
0.9728967744
1.3933612675
0.6871793419
-1.4752153205
-0.2654243822
$-0.3256592168$
0.4306635915
$-0.3869378698$
$-1.7998421963$
$-1.7140836726$
2.4213788941
1. 6978160485
$-0.1165551285$
0.0770713767
2.8362532973
2. 4886808542
2.9974896595
2.5462638519
1. 9746639410
1. 5955079845
3. 0183243554
4.7160255202
4.5501878225
4.0385803864
$-2.3433198986$
$-2.2689308326$
$-1.8832288234$
$-2.4123876033$
$-0.7206229054$
0.9294867639
$-0.3731519572$
$-0.5698351506$
$-0.0131669057$
1. 1154840610
3.5285826773
3.6465463633
4.4463639843
4.9179327790
5.2484385015
1. 7747943510
3. 3554905704
1. 7549051542
0.5411431172 
49-Me$$
\text { C }
$$

C

C

C

C

C

$\mathrm{Ru}$

$\mathrm{Cl}$

C

$\mathrm{N}$

C

C

$\mathrm{N}$

C

$\mathrm{Cl}$

C

50-Me

$$
\begin{array}{r}
4.8664932658 \\
5.3815947712 \\
-3.0518752915 \\
-3.6316570437 \\
-3.3319739058
\end{array}
$$

$$
\begin{array}{r}
-1.4121966714 \\
-0.9573743942 \\
-1.1376614338 \\
-0.6328931495 \\
0.5919447011
\end{array}
$$

2.7614799470

1.1339340251

3.2856693720

4.8674044624

3.6031388327
3.4860175370

2. 6266832150

3. 3944212030

4.8512454902

4.9250347413

1. 1916338300

$-0.2867898544$

0.7498799058

$-0.7632534771$

$-0.2506904516$

$-1.0424137184$

$-1.7958270935$

$-1.7281245535$

0.5657956872

$-2.3415629950$

$-1.6682647173$

0.2877774350

$-0.7584727150$

$-2.1912826319$

$-1.7275669254$

$-0.3932689016$

$-1.2899056555$

$-2.8340269105$

$-0.0500954070$

1. 2921245734

1.1032340355

$-3.3799457476$

$-1.7961355648$

$-2.3408808462$

0.9303376163

3.1762755210

0.0249549779

$-0.4619682362$

1. 2586629425

5.3063606010

5.5826205702

3.0043332628

3. 2913608984

5.0467572178

5.5909536536

$-2.8945357185$

$-2.3477648275$

$-2.4372912315$
1.8034550735

0.7759615588

$-0.5218987562$

$-0.0463043414$

1. 3634309386

0.9968949979

$-0.1459308214$

$-2.3794465819$

$-0.5488879310$

0.0144647009

$-0.3410511327$

$-1.5850007023$

$-1.4161379685$

1.2191260622

$-2.4002742470$

1. 8856509765

0.4958334287

$-0.4633792789$

$-0.1265680102$

0.4818755703

$-0.5336408148$

$-2.5165001126$

$-1.6252689869$

2.1193716597

1.1810246944

1.2566376736

$-2.5569919716$

$-3.3507337399$

$-2.0315240041$

2.0607063859

2. 8280848234

1.5297914445

$-1.4964693867$

0.1601320833

1. 3238798620

2. 0546931044

$-1.1229225640$

$-1.1548028168$

0.0386119562

$-0.7399471437$

$-0.7814219696$

$-0.2924357540$

0.9075275774
2.4006897687

2. 6151177417

2. 8127362888

3. 0405270112

2.3990024294

2. 5570875012

2. 2588355030

2. 3256259007

0.2962347021

$-0.8148967099$

$-2.0054467723$

$-1.5204347272$

$-0.0615278913$

$-0.8401330553$

0.8165438352

2.0093823185

4.4366353054

4. 3712136411

4.7160717202

$-2.2563009550$

$-2.8646674342$

$-1.8139070490$

$-1.8635867461$

$-0.7237506803$

$-0.0289095545$

$-1.7931347209$

0.5045996400

0.7955384183

1.8433775919

2.5214711806

2.2082684382

4.6449082151

4.5462647966

4.7866539776

1.3656549753

2. 9414913182

3. 6388112419

1. 9239440188

4.1163872064

2. 6292698887

4.1878497947

5.7928804501

4.4698057596
3. 3352820000

2. 5121730000

3. 2999660000
-0.4398050000
0.7698260000
1.6899370000

3. 6313460000

3. 2152220000

2. 6131850000 
4.7384190000

4.7964900000

1.0684150000

0.2889490000

$-1.0340710000$

$-2.3405660000$

$-0.2908520000$

0.7730390000

$-0.8390200000$

$-1.8444680000$

$-1.8260950000$

$-0.9487960000$

$-0.2163520000$

$-2.5515800000$

0.7161580000

$-1.5401970000$

$-1.5497930000$

$-0.2582200000$

$-1.3725550000$

$-2.8392370000$

0.1842570000

1.3222050000

1.3814340000

$-3.5682030000$

$-2.0345390000$

$-2.6063130000$

0.7808190000

2.9380780000

$-0.0512510000$

$-0.8889010000$

0.9710910000

4.9784570000

5.4477940000

3.0994450000

3.1244700000

5.1404490000

5.4878980000

$-3.1870460000$

$-2.4003930000$

$-2.4578790000$
1.2520690000
0.0636350000
0.9739220000
0.2658290000
$-0.5580540000$
0.0030690000
$-0.1596860000$
$-2.3616050000$
$-0.5517640000$
$-1.3530810000$
$-1.5653420000$
$-0.3968370000$
$-0.0568760000$
$-2.2764080000$
1.0617280000
1.9499950000
0.4639420000
$-0.6711150000$
$-2.5404880000$
$-1.5415050000$
2.0203140000
1.0153450000
0.9832740000
$-2.4188670000$
$-3.2417180000$
$-1.8487650000$
2.0206370000
2.6325710000
1.0800360000
$-1.6127260000$
$-0.4162090000$
0.9341720000
2.0526280000
$-0.7900790000$
$-1.2826920000$
0.4284520000
$-0.7233020000$
$-0.5362620000$
$-0.1399580000$
1.0620430000

2.5203740000

3.5143200000

3. 3770050000

4.5528120000

4.1925180000

4.7120310000

2.3897350000

2.4079780000

0.4794460000

0.0969140000

$-1.3597860000$

$-1.8335380000$

$-0.6025360000$

0.9713770000

$-0.5807610000$

2.0708460000

$-2.1603100000$

$-2.6363570000$

$-1.5888990000$

$-1.7715400000$

$-0.6001450000$

0.3260380000

$-1.4466090000$

0.5908900000

1.0299480000

1.9732880000

3.2790260000

2.2085980000

5.1967710000

4.4378780000

5.0590800000

1.4924610000

2.7669760000

4.6433620000

2.9634380000

4.4896520000

3.1969600000

4.2701970000

5.8024160000

4.4762880000

\section{1-Me}

$\begin{array}{lr}\mathrm{C} & -0.5529005063 \\ \mathrm{C} & -1.3692177149 \\ \mathrm{C} & -2.5988796293 \\ \mathrm{C} & -2.7906836682 \\ \mathrm{C} & -1.6077900513 \\ \mathrm{C} & -0.9395041010 \\ \mathrm{C} & 0.3287683058 \\ \mathrm{Ru} & 0.1277294590 \\ \mathrm{C} & 1.4707401889 \\ \mathrm{C} & 1.4869480234 \\ \mathrm{C} & 0.1073746283 \\ \mathrm{~N} & -0.9180184127 \\ \mathrm{C} & -0.7307796266 \\ \mathrm{C} & 0.7748246632 \\ \mathrm{~N} & 1.0747096100\end{array}$

$$
\begin{array}{r}
-3.1524616445 \\
-1.9985831030 \\
-2.4177088894 \\
-3.8969014888 \\
-4.2705042254 \\
-0.6005390483 \\
-0.1122731405 \\
-0.3549984436 \\
0.7769542546 \\
2.2674689569 \\
-0.6310862023 \\
-1.2157518241 \\
-1.1689403067 \\
-0.9120380993 \\
-0.3419602525
\end{array}
$$


$-2.2505704205$

2. 4519901158

1.7198037326

$-1.5421204118$

1.0434043767

1. 3414185258

$-1.0458472219$

$-1.3218575134$

2. 4716394781

3.0490095917

2. 8792185219

$-2.6964545278$

$-2.1879255297$

$-2.8865837773$

2.4283172343

0.3729099349

$-1.7465610556$

1.0907943395

$-3.3603978871$

0.2325773557

$-0.0476328012$

$-3.7710768567$

$-2.7292530651$

$-1.2057950498$

$-1.9561382497$

2.0363815982

2.0467168579

0.4941494388

$$
\begin{array}{r}
-1.4348534565 \\
-0.0115323650 \\
-2.2297544243 \\
1.4370370932 \\
-0.2144849564 \\
-1.8444628317 \\
-2.1067255105 \\
-0.3448628569 \\
0.5944662133 \\
-0.9156919555 \\
0.5652239552 \\
-2.2955972890 \\
-1.6589712258 \\
-0.5528775194 \\
0.2875880251 \\
0.8952975633 \\
0.1247066686 \\
-0.8058929117 \\
-1.7723283770 \\
-3.4386082911 \\
-2.8822856908 \\
-4.1476543446 \\
-4.4280147767 \\
-5.2671888628 \\
-4.2669386770 \\
2.5673187535 \\
2.6814355162 \\
2.7037189238
\end{array}
$$
0.2923993976
$-0.1628842415$
2.5358197944
2.0463372176
$-2.6110632915$
$-1.9525063124$
$-2.1745199671$
$-2.1333319663$
0.7414333340
0.0080056500
$-0.9895200047$
$-0.2157013563$
1.3570024845
0.1598748332
3.3081329385
5.1806682566
4.3139169515
5.1222291040
3.6740506832
4.3348726920
5.9796960812
4.7407212879
3.3514636994
5.0412229657
6.2878105447
4.1327895660
2.3798577449
3.2179511103

\section{8-OMe}

$\begin{array}{lr}\mathrm{C} & 3.6649922949 \\ \mathrm{C} & 2.6686877053 \\ \mathrm{C} & 3.2562450564 \\ \mathrm{C} & 4.7535695957 \\ \mathrm{C} & 5.0312947151 \\ \mathrm{C} & 1.2835594607 \\ \mathrm{Ru} & -0.2690749396 \\ \mathrm{Cl} & 0.4919497631 \\ \mathrm{C} & 0.2020707122 \\ \mathrm{~N} & 0.7725704204 \\ \mathrm{C} & 0.8023000947 \\ \mathrm{C} & 0.3714577983 \\ \mathrm{~N} & -0.0895708401 \\ \mathrm{C} & 1.0246367364 \\ \mathrm{C} & -0.7403995560 \\ \mathrm{Cl} & -1.8112928258 \\ \mathrm{C} & -0.7372135874 \\ \mathrm{C} & -1.9190245616 \\ \mathrm{O} & -3.0595920547 \\ \mathrm{C} & -4.1204452317 \\ \mathrm{H} & 0.1018705849 \\ \mathrm{H} & 1.8037588211 \\ \mathrm{H} & 1.2036414816 \\ \mathrm{H} & -0.4346227818 \\ \mathrm{H} & 0.4284447682 \\ \mathrm{H} & 0.7295276759 \\ \mathrm{H} & 2.0872401377 \\ & \end{array}$
2.9572959650
2.4462042379
1.5891836149
1.9884472065
2.5349248663
2.7654129688
2.3068545690
3.0086782044
0.3319586287
$-0.4675546240$
$-1.8821998449$
$-1.8288714180$
$-0.4432636320$
$-0.1706036168$
$-0.0688641784$
1.6562409900
4.8248981781
4.4755283765
4.4498455784
3.6541348062
$-2.4549483059$
$-2.3054246868$
$-2.0183365507$
$-2.5318884398$
$-0.8442737077$
0.8497600367
$-0.3162876035$ 
$-1.6629599051$

$-0.0786304759$

$-0.9602901795$

1.1425229455

3.5002497562

$-0.6926634046$

$-1.9994410142$

0.1014836191

5.4330997205

5.7606034305

2.7584198545

3.1278611838

4.9103154098

5.4170609611

$-4.1871681151$

$-5.0405579222$

$-3.9584547379$

$$
\begin{array}{r}
-2.6128056710 \\
-3.3406885982 \\
-2.4905091557 \\
1.7555580456 \\
2.3278426340 \\
1.3702847512 \\
-1.3239419387 \\
-0.3274907162 \\
1.6627610013 \\
1.0101731299 \\
-1.3825663102 \\
-0.1839674335 \\
-1.1590128619 \\
-0.7061205817 \\
-1.1705031755 \\
0.3822120305 \\
0.1847799477
\end{array}
$$

$-0.1640856004$

$-0.7023118510$

$-1.6302002203$

$-1.6444547419$

$-0.8956496991$

C

$\mathrm{Ru}$

$\mathrm{Cl}$

C

C

C

C

C

C

C

C

C

$\mathrm{Cl}$

$\mathrm{H}$

$\mathrm{H}$

$\mathrm{H}$

$\mathrm{H}$

$\mathrm{H}$

$\mathrm{H}$

$\mathrm{H}$

$\mathrm{H}$

$\mathrm{H}$

$\mathrm{H}$

$\mathrm{H}$

$\mathrm{H}$

$\mathrm{H}$

$\mathrm{H}$

$\mathrm{H}$

$\mathrm{H}$

$\mathrm{H}$
$-0.2910115436$

$-1.7337835647$

$-2.2606588597$

0.6111292703

1.1158547250

2. 5594288748

3. 3757086822

4.8317186661

4.8230526919

3. 3728567076

0.3358181085

$-0.9578702398$

0.7183715714

$-1.5852345497$

$-0.2064009529$

$-1.1146296805$

$-2.6694837824$

$-0.0441627450$

1.2757728287

1.2133392417

$-3.2786530878$

$-1.6934726244$

$-2.3197024232$

0.8037218029

3.0196838366

0.3952318107

$-1.0785623690$

$-2.0093659927$

1.1613720775

5.1523453751

5.4842591091

3. 0585936914

3.2343508668
0.0080866844

$-0.5782817387$

$-1.4667321610$

$-1.6209279205$

$-0.3597510344$

$-0.1933065527$

1.7826091792

$-2.4685137393$

1.2396125320

1.0067437019

0.8443829410

$-0.4383599312$

0.0508413600

1. 5519652499

1. 9262208402

0.1897641803

$-0.3705648131$

$-2.4288902901$

0.4508332856

$-0.5347689184$

$-2.5413768961$

$-1.6742865502$

2.1173748961

1. 2425478069

1.2823348604

$-2.6493206049$

$-3.4064872520$

$-2.1022906120$

2. 0533175295

2.9546126536

1.1907890475

$-1.4565823494$

0.3627287405

$-0.4864173126$

1.7098970998

2.1659396598

$-1.1747127313$

$-0.9286628219$
$-0.6519059130$
$-0.2737769086$
0.9954972531
3.4731078850
3.5892829956
5.0635886857
4.2782179109
5.0644645633
1.7553043838
3.3549187058
1.7694545513
0.5252445574
2.7927330146
1.1623073608
3.7696646841
4.0159453275
2.6048109583
$-0.8026681387$
0.2843677427
$-0.1194949173$
$-1.5812142555$
$-2.0273969131$
2.2675805822
1. 9178098655
0.7249585163
$-0.7914303908$
2.6417436238
2.6313915349
2.5781975504
2.7886182365
2.3991575723
2.5411929880
4.4938868252
4.3980910714
2. 4778455362
$-2.3048930718$
$-2.8588266431$
$-1.8674958715$
$-1.9609102792$
$-0.7346834569$
0.0710209672
$-1.7050248968$
0.3623317211
0.7291003901
1.7507855685
2.7340881187
2.5242843494
4.9111342379
4.4282613433
4.8286770364
4.6816483297
1.3591303079
3.0241657209
3.3200404343
1.6066390925 


$\begin{array}{lr}\mathrm{H} & 5.1009644316 \\ \mathrm{H} & 5.5595415022 \\ \mathrm{C} & -3.2983627513 \\ \mathrm{H} & -4.0202905457 \\ \mathrm{H} & -3.4752419368 \\ \mathrm{H} & -3.3858229556\end{array}$

$$
\begin{array}{r}
-0.0445818467 \\
-0.5316654187 \\
-0.0884866042 \\
0.4041986597 \\
0.2076090658 \\
-1.1787657451
\end{array}
$$
3.8472783755
2. 2154152729

\begin{tabular}{|c|c|}
\hline $\mathrm{N}$ & -1.8000410000 \\
\hline $\mathrm{C}$ & -0.8130150000 \\
\hline $\mathrm{N}$ & -0.1794440000 \\
\hline C & -0.8862860000 \\
\hline $\mathrm{C}$ & -1.7606220000 \\
\hline $\mathrm{Ru}$ & -0.3169200000 \\
\hline $\mathrm{Cl}$ & -1.5781450000 \\
\hline $\mathrm{C}$ & 0.7340920000 \\
\hline $\mathrm{C}$ & -2.5046310000 \\
\hline $\mathrm{C}$ & 1.0423130000 \\
\hline $\mathrm{C}$ & 0.2914190000 \\
\hline C & -1.0723730000 \\
\hline O & -2.1306080000 \\
\hline $\mathrm{C}$ & -3.4097850000 \\
\hline $\mathrm{C}$ & 2.4901620000 \\
\hline C & 3.2663400000 \\
\hline C & 4.7102890000 \\
\hline $\mathrm{C}$ & 4.7827890000 \\
\hline C & 3.3278100000 \\
\hline $\mathrm{Cl}$ & 0.7175430000 \\
\hline $\mathrm{H}$ & -1.4890800000 \\
\hline $\mathrm{H}$ & -0.1795200000 \\
\hline $\mathrm{H}$ & -1.2985690000 \\
\hline $\mathrm{H}$ & -2.7682920000 \\
\hline $\mathrm{H}$ & 0.1866270000 \\
\hline $\mathrm{H}$ & 1.3120250000 \\
\hline $\mathrm{H}$ & 1.4246990000 \\
\hline $\mathrm{H}$ & -3.5465180000 \\
\hline $\mathrm{H}$ & -2.0336490000 \\
\hline $\mathrm{H}$ & -2.4808790000 \\
\hline $\mathrm{H}$ & 0.7395300000 \\
\hline $\mathrm{H}$ & 2.8926570000 \\
\hline $\mathrm{H}$ & -0.0086140000 \\
\hline $\mathrm{H}$ & -1.0853410000 \\
\hline $\mathrm{H}$ & 0.9586010000 \\
\hline $\mathrm{H}$ & 4.9553610000 \\
\hline $\mathrm{H}$ & 5.4093500000 \\
\hline $\mathrm{H}$ & 3.0969360000 \\
\hline $\mathrm{H}$ & 3.1261270000 \\
\hline $\mathrm{H}$ & 5.1225190000 \\
\hline $\mathrm{H}$ & 5.4837850000 \\
\hline $\mathrm{H}$ & -4.1276300000 \\
\hline $\mathrm{H}$ & -3.6471150000 \\
\hline $\mathrm{H}$ & -3.4540400000 \\
\hline
\end{tabular}
4. 4179777662
5.0719159162
3. 3794497867
4.5331590660

\section{0-OMe}

$$
\begin{aligned}
& -1.3719650000 \\
& -0.5622400000 \\
& -0.0393850000 \\
& -0.3632010000 \\
& -1.5478100000 \\
& -0.1735300000 \\
& 1.9120410000 \\
& \text { 1. } 0940790000 \\
& -2.3342620000 \\
& 0.9579370000 \\
& 0.2253710000 \\
& -0.4940370000 \\
& 0.1975340000 \\
& -0.3330630000 \\
& 0.7836000000 \\
& \text { 1. } 7425880000 \\
& \text { 1. } 3280450000 \\
& 0.0895570000 \\
& -0.4365240000 \\
& -2.3951810000 \\
& 0.4987140000 \\
& -0.6153840000 \\
& -2.5148400000 \\
& -1.5206360000 \\
& 2.0433610000 \\
& 1.0547630000 \\
& 1.0288130000 \\
& -2.4030670000 \\
& -3.3233360000 \\
& -1.9980870000 \\
& 2.0030200000 \\
& 2.6996430000 \\
& 1.0229070000 \\
& -1.5628010000 \\
& -0.5048410000 \\
& 1.0688010000 \\
& 2.1231740000 \\
& -0.8457270000 \\
& -1.2459690000 \\
& 0.4067240000 \\
& -0.6712940000 \\
& 0.1864060000 \\
& -0.1385500000 \\
& -1.4134130000
\end{aligned}
$$




\section{1-OMe}

\begin{tabular}{|c|c|c|c|}
\hline $\mathrm{N}$ & -0.9614053619 & -1.2226833239 & -0.2203761550 \\
\hline C & 0.0001540374 & -0.5450925595 & 0.4336515956 \\
\hline $\mathrm{N}$ & 0.8785984717 & -0.0589876925 & -0.4558007499 \\
\hline $\mathrm{C}$ & 0.6076442354 & -0.5657028966 & -1.8103965998 \\
\hline $\mathrm{C}$ & -0.8420437243 & -1.0513631590 & -1.6766110557 \\
\hline $\mathrm{Ru}$ & 0.0832244952 & -0.3467692868 & 2.4753542679 \\
\hline $\mathrm{Cl}$ & 1.6931670729 & -2.2102693306 & 2.4682739718 \\
\hline $\mathrm{C}$ & 2.1730294597 & 0.5155912394 & -0.1199911247 \\
\hline $\mathrm{C}$ & -2.2259571294 & -1.6338343096 & 0.3692622161 \\
\hline $\mathrm{C}$ & -0.9747127374 & -0.7240389213 & 4.2849428143 \\
\hline C & 0.2772929929 & -0.1059822763 & 4.7332462009 \\
\hline C & -1.3199938581 & -2.1300081384 & 4.4988449894 \\
\hline C & -2.5511558607 & -2.6245938045 & 4.2418401759 \\
\hline $\mathrm{C}$ & -2.6543261506 & -4.0981520224 & 4.5264722026 \\
\hline C & -1.3780549175 & -4.3770059498 & 5.3614701266 \\
\hline $\mathrm{C}$ & -0.4026466271 & -3.2216799874 & 5.0232964621 \\
\hline $\mathrm{Cl}$ & -1.5581315544 & 1.4580631604 & 2.1427926122 \\
\hline $\mathrm{C}$ & 1.3871032790 & 0.6731368044 & 3.4012148189 \\
\hline O & 1.4166473372 & 2.0159304322 & 3.4791067384 \\
\hline $\mathrm{C}$ & 2.5480546200 & 2.5866142532 & 4.1488229199 \\
\hline $\mathrm{H}$ & 0.7321241153 & 0.2243164192 & -2.5561487670 \\
\hline $\mathrm{H}$ & 1.2958401996 & -1.3892266369 & -2.0482360364 \\
\hline $\mathrm{H}$ & -1.0332199940 & -1.9931613423 & -2.1993253347 \\
\hline $\mathrm{H}$ & -1.5618319604 & -0.2984488139 & -2.0275051107 \\
\hline $\mathrm{H}$ & 2.0693221229 & 1.1147710679 & 0.7850338551 \\
\hline $\mathrm{H}$ & 2.9295208193 & -0.2644301420 & 0.0463600497 \\
\hline $\mathrm{H}$ & 2.4911186339 & 1.1690405649 & -0.9401886945 \\
\hline $\mathrm{H}$ & -2.6212093749 & -2.4820505562 & -0.1986201243 \\
\hline $\mathrm{H}$ & -2.0635473561 & -1.9574661955 & 1.3993242223 \\
\hline $\mathrm{H}$ & -2.9507625238 & -0.8118210926 & 0.3673549229 \\
\hline $\mathrm{H}$ & 2.3583174955 & 0.2019495668 & 3.6087979318 \\
\hline $\mathrm{H}$ & 0.1228852090 & 0.8471969006 & 5.2384940371 \\
\hline $\mathrm{H}$ & -1.8313942213 & -0.0544964568 & 4.2498614727 \\
\hline $\mathrm{H}$ & 0.9866673785 & -0.7796408532 & 5.2069395383 \\
\hline $\mathrm{H}$ & -3.3750983968 & -2.0361181785 & 3.8463128948 \\
\hline $\mathrm{H}$ & 0.3279240128 & -3.4970508700 & 4.2543082884 \\
\hline $\mathrm{H}$ & 0.1740839409 & -2.8983668720 & 5.8979817773 \\
\hline $\mathrm{H}$ & -3.5789051193 & -4.3702183874 & 5.0519594756 \\
\hline $\mathrm{H}$ & -2.6553034737 & -4.6701295742 & 3.5856149925 \\
\hline $\mathrm{H}$ & -0.9460113794 & -5.3599424856 & 5.1568506238 \\
\hline $\mathrm{H}$ & -1.6338744717 & -4.3466893358 & 6.4259669431 \\
\hline $\mathrm{H}$ & 2.5131995495 & 3.6583389439 & 3.9497782905 \\
\hline $\mathrm{H}$ & 2.4854617902 & 2.4130092988 & 5.2304347173 \\
\hline $\mathrm{H}$ & 3.4844586935 & 2.1669073348 & 3.7640372274 \\
\hline
\end{tabular}




\section{Disfavored Intramolecular Pathway}

53

$\begin{array}{lr}\mathrm{N} & -1.2214345540 \\ \mathrm{C} & -0.1042611604 \\ \mathrm{~N} & 0.8629910203 \\ \mathrm{C} & 0.4391584609 \\ \mathrm{C} & -1.0830598208 \\ \mathrm{Ru} & -0.1222895088 \\ \mathrm{C} & -0.7799624341 \\ \mathrm{C} & -1.4259707096 \\ \mathrm{C} & 2.2832043914 \\ \mathrm{C} & -2.5461065095 \\ \mathrm{Cl} & -1.3183263473 \\ \mathrm{Cl} & 1.2503660184 \\ \mathrm{C} & 1.3865937475 \\ \mathrm{H} & 0.8862150158 \\ \mathrm{H} & 0.7590268834 \\ \mathrm{H} & -1.5892207492 \\ \mathrm{H} & -1.5230733145 \\ \mathrm{H} & 2.7776406038 \\ \mathrm{H} & 2.4201026791 \\ \mathrm{H} & 2.7273802679 \\ \mathrm{H} & -3.1180800527 \\ \mathrm{H} & -3.0701717554 \\ \mathrm{H} & -2.4664160735 \\ \mathrm{H} & 2.3294512510 \\ \mathrm{H} & 1.3463972720 \\ \mathrm{H} & -2.1029676984 \\ \mathrm{C} & -0.3249523245 \\ \mathrm{C} & -1.1422336726 \\ \mathrm{H} & 0.7389546836 \\ \mathrm{H} & -0.3635911959 \\ \mathrm{C} & -0.6558499674 \\ \mathrm{H} & -2.2038155685 \\ \mathrm{H} & -1.0680340024 \\ \mathrm{C} & -1.4727285493 \\ \mathrm{H} & 0.3996629878 \\ \mathrm{H} & -0.7023937441 \\ \mathrm{C} & -0.9967230772 \\ \mathrm{H} & -2.5358838258 \\ \mathrm{H} & -1.6362879836 \\ \mathrm{H} & \\ \mathrm{H} & -0559443132\end{array}$

0.2132602082

$-0.0751324478$

$-0.4372678305$

$-0.2434098114$

$-0.1326679574$

0.1758957725

1.4936684503

0.4216793995

$-0.5401205013$

0.4070429880

$-2.0023067400$

2.1484788372

$-0.7041942618$

0.6803505049

$-1.0831175627$

$-1.0874987903$

0.6411652416

0.4320104813

$-0.8858344423$

$-1.2670661082$

1.0687103283

$-0.5472601843$

0.8861845903

$-0.1635708937$

$-1.7610443167$

$-0.3429654049$

2.8339055450

3. 4041968443

2.7741309222

3. 5061199112

4. 8139435078

3. 4423467250

2. 7360104815

5.4288831805

4.7710223077

5.4570948822

5.8021901484

5.5591281668

6.2385491320

5. 6887707165
$-0.5904360459$

0.1023479549

$-0.7539270493$

$-2.1526019923$

$-2.0126115450$

2.1728934878

3. 8209246605

3. 8508117573

$-0.4695487195$

$-0.0194080122$

2.1182204300

1.5562803538

2. 6737947071

$-2.5467254243$

$-2.7762119103$

$-2.2149855634$

$-2.6486505521$

$-0.5830502147$

0.5539160277

$-1.1572137015$

$-0.6781256107$

0.1048561550

0.9587599734

2. 8020908952

2.9525784468

4.1804627303

4.2326956905

5.4074731646

4.4948214337

3.3682478773

5.7966184968

5.1287340717

6.2741866499

6.9007529696

6.0965584644

4. 9039485512

8.0897583320

6.6893921783

8.8526146034

8.3430657594

54

C $\quad-0.0360025643$

C $\quad-1.4413704818$

$\mathrm{N} \quad-1.5574061914$

C $\quad-0.3515821433$

0.5822804715

$-2.7609358002$

2. 1545238261

$-1.6133842775$

1. 5428254593

$-1.6352508604$

1.0262256389

0.9085693857

$-0.2637663806$

0.3276198905

1. 4580852364

$-0.4716061795$

0.3166845087

0.1430407918

0.0751502240

2.1780094719

2. 3502732348

3.0784434010

1.8364623433

1. 9261902306

1.6126578763

$-0.3073331286$

$-0.0621294252$

2. 6073184618

4. 3548609934 
$-0.5745739475$

$-0.3635354394$

$-0.7448573433$

$-0.4785530194$

$-0.8783284018$

$-0.0404614936$

$-2.1443182263$

3. 2363450653

1. 9769234803

0.7156766326

2.2721864398

2.7903593604

1.0651869929

1. 9170969913

0.9063724351

$-0.6823701792$

0.2058466509

0.4247266259

$-1.2838551905$

$-1.2821913682$

0.6950070694

$-0.9526269235$

$-1.8044132728$

$-0.1664461203$

0.5861355836

$-1.0380105649$

$-1.9368501726$

$-0.3826562703$

1. 0227420195
4.1342112995

4.3597765021

5.7858736087

5.9924432717

7.3639268857

8.2721051025

1.2297687377

$-1.4178799948$

$-2.5330200233$

$-2.3556242349$

$-1.8485787590$

0.4792969244

0.5890229637

$-0.9549560600$

$-0.1535330953$

$-0.3046602271$

1. 2288403100

3. 1123198696

2.3706319960

4.8559482379

4.1714625925

3. 6213083754

5.9638736592

6.5144861086

5.8248392790

5.2301839070

7. 6116280170

9.2523890304

8.0666518383

55

$\begin{array}{lr}\mathrm{N} & 1.1531040983 \\ \mathrm{C} & 0.9387406725 \\ \mathrm{C} & -0.4367045591 \\ \mathrm{~N} & -0.9466560143 \\ \mathrm{C} & 0.0224467956 \\ \mathrm{C} & -2.2029590925 \\ \mathrm{Ru} & -0.1713771210 \\ \mathrm{C} & 0.7021112876 \\ \mathrm{C} & 0.8946966622 \\ \mathrm{C} & -0.1120416042 \\ \mathrm{C} & 2.4880678760 \\ \mathrm{Cl} & 1.3222321813 \\ \mathrm{Cl} & -2.3636345045 \\ \mathrm{H} & 0.9433229239 \\ \mathrm{H} & 1.7342357984 \\ \mathrm{H} & -0.3653640440 \\ \mathrm{H} & -1.1091874807 \\ \mathrm{H} & 3.1952719532 \\ \mathrm{H} & 2.8006312547 \\ \mathrm{H} & 2.5125397066 \\ \mathrm{H} & -2.9825508149 \\ \mathrm{H} & -2.1007445895 \\ \mathrm{H} & -2.5052140242 \\ \mathrm{C} & 1.0742248845 \\ \mathrm{H} & 1.9284432222 \\ \mathrm{H} & 0.0984139244\end{array}$

-0.0699782397
-0.4121544868
-1.0883514489
-0.7006646453
-0.1814443477
-1.2987367239
0.2523406744
1.8524625249
2.0049252905
2.0104619773
0.2992653644
-1.5145468285
1.2358351175
0.4971523806
-1.0731445111
-2.1837781134
-0.7401179676
-0.4781315331
1.2521953743
0.3687896488
-1.0624888296
-2.3915272487
-0.8887557649
2.9332841811
2.0137497453
2.0237625301
$-0.8150597152$

$-2.2257882969$

$-2.1903127205$

$-0.8686493247$

$-0.0721826512$

$-0.4404613452$

1. 8603366323

2.0189144258

3. 4714992125

4. 3679414422

$-0.3834368589$

2.4139414780

1.7596459746

$-2.8435051161$

$-2.5859690927$

$-2.2565582065$

$-2.9810706534$

$-0.6967050419$

$-0.8321190369$

0.7012733414

$-1.1728717040$

$-0.3725704230$

0.5200389301

1.0519371597

3. 8259918368

5.4341938194 


$\begin{array}{lr}\mathrm{H} & -1.1519720992 \\ \mathrm{C} & 2.4310936448 \\ \mathrm{H} & 1.0219122415 \\ \mathrm{H} & 0.2718206802 \\ \mathrm{C} & 2.7257652543 \\ \mathrm{H} & 2.4416496743 \\ \mathrm{H} & 3.2362252489 \\ \mathrm{C} & 4.0767978483 \\ \mathrm{H} & 2.6725655323 \\ \mathrm{H} & 1.9432418104 \\ \mathrm{C} & 4.2862572767 \\ \mathrm{H} & 4.9337056293 \\ \mathrm{H} & 5.2868722472 \\ \mathrm{H} & 3.4620025886\end{array}$

56

$$
\text { C }
$$$$
\begin{aligned}
& \mathrm{C} \\
& \mathrm{N} \\
& \mathrm{C} \\
& \mathrm{C} \\
& \mathrm{N} \\
& \mathrm{C} \\
& \mathrm{C}
\end{aligned}
$$

0.2423368725

1. 4577867617

1. 3741017482

$-0.0240946823$

$-0.6668908554$

2.7452919602

$-2.0131854714$

$\mathrm{Ru} \quad-0.1617987667$

$\mathrm{Cl} \quad-2.2358990603$

C $\quad 0.8255846297$

C $\quad 1.0621579159$

C $\quad 0.3828306047$

Cl 1.1900112223

$\mathrm{H} \quad 1.4745072031$

$\mathrm{H} \quad 2.1731242703$

0.0065845769

$-0.5778326859$

3. 4826390837

3. 0882330191

2.6720337514

$-2.6427015087$

$-2.0088815308$

$-2.4308694919$

1.1053512364

1.7846745493

0.5561493827

$-0.3849648620$

2. 4247488101

1. 0284924585

0.2563705538

2. 5865815139

2. 4538049328

3. 2787246737

3. 8472431612

2. 5718871548

1. 7141518371

4. 8199133935

3.9522796364

5.7118910608

4.7577458623
2.0290263779

3. 6175325381

2. 5636795604

3. 6862142629

4. 7431594888

4. 0351381429

2. 8711247547

5.3721849572

4. 3251091282

5.5106753391

6.6510982803

4.7035853598

7.0475124544

7.3525447935
4.0557620844

1.3223983603

0.0237917709

1.1447845623

0.3117843516

2.3362459003

1. 2821618723

0.5267133369

$-0.7059950249$

0.3766527863

0.8428360601

0.4233970770

0.9944989684

0.9590872596
$-0.4041595103$
0.0443975111
$-0.4408922888$
$-0.5571605525$
$-0.8717422390$
$-1.9575037895$
$-1.4932706455$
$-0.9179568372$
$-2.0145442531$
$-0.8256824885$
$-0.0434733369$
$-1.3674589698$
0.1826830120
1. 3424397705
1. 7225196808
2. 0321303490
$-0.0197735772$
$-0.4991014391$
1.8889846742
1.5251714569
2. 0021840138
3. 4318829513
2. 9619384840
4.1238154647
2.8246528462
$-1.5359592914$
$-0.0073996341$
$-2.6302159046$
$-1.5828098012$
$-2.5900743227$
$-2.1903594272$
$-1.9319330943$
$-1.2303078657$
$-2.9211488931$
$-0.8212580381$
$-0.2511413320$
0.9013590644
0.0536623546
$-0.4630710444$
1.0611667238
$-1.2652015997$
$-1.3891895017$
$-2.4230041900$
$-0.7435041489$
$-0.1899308151$
0.2891805591
2. 7498911637
0.9428482664
1. 3957216081
3.9457003126
3. 1089863517
5.1863131088
3. 5745023897
3.6591153672
3. 5346803580
1.0889059658
2. 2987658053
3. 4552869800
4. 5983118922
4.0176370620
$-0.0512888254$
1. 0111905128
$-0.0147001656$
2.0727062568
2. 8461747092
1. 0537781771
5.4062894859
0.1377313585
4.1185730052
5.2694767122
5.4905189160
$-1.0027115868$
0.0197509446
5.9477255174
$-0.7707384700$
1.0794426964
6.0883256339
$-0.6030394569$
4. 9664986814
$-1.7226569165$ 


\begin{tabular}{|c|c|c|c|}
\hline $\mathrm{N}$ & 1.2686319613 & -0.0979787543 & -0.6502659724 \\
\hline C & 1.2663324682 & -0.5227817658 & -2.0551874418 \\
\hline $\mathrm{C}$ & -0.0370084061 & -1.3214665420 & -2.142939358 \\
\hline $\mathrm{N}$ & -0.7702956993 & -0.8500807253 & -0.959971073 \\
\hline $\mathrm{C}$ & 0.0501707886 & -0.2344167048 & -0.068508825 \\
\hline $\mathrm{C}$ & -2.0561780635 & -1.4699604156 & -0.670160884 \\
\hline$u$ & -0.4280399840 & 0.2498490270 & 1.78707324 \\
\hline $\mathrm{C}$ & 0.3031829627 & 1.9534734730 & 1.85842577 \\
\hline $\mathrm{C}$ & 0.5888280579 & 2.3706284288 & 3.229061000 \\
\hline $\mathrm{C}$ & 1.1716742363 & 3.5284514537 & 3.6013196 \\
\hline $\mathrm{C}$ & 2.4865063889 & 0.4646127040 & -0.09450885 \\
\hline $\mathrm{Cl}$ & 1.1705545376 & -1.2823980533 & 2.6343 \\
\hline $\mathrm{Cl}$ & -2.7171450849 & 0.9131084813 & 1.6670651 \\
\hline $\mathrm{H}$ & 1.2654685828 & 0.3542641434 & -2.7190 \\
\hline $\mathrm{H}$ & 2.1559105466 & -1.1205044943 & -2.27796462 \\
\hline $\mathrm{H}$ & 0.1355044893 & -2.4056582279 & -2.0704 \\
\hline $\mathrm{H}$ & -0.6063303192 & -1.1228933017 & -3.05626225 \\
\hline $\mathrm{H}$ & 2.7196576328 & 1.4334398961 & -0.5572 \\
\hline $\mathrm{H}$ & 2.3793927010 & 0.5820141616 & 0.98 \\
\hline $\mathrm{H}$ & 3.3170343072 & -0.2232983305 & $-0.2 \varepsilon$ \\
\hline $\mathrm{H}$ & -2.6722497449 & -1.4361978198 & -1.57 \\
\hline $\mathrm{H}$ & -1.9252543500 & -2.5211740598 & -0.37388591 \\
\hline $\mathrm{H}$ & -2.5647762990 & -0.9188795207 & 0.1184 \\
\hline $\mathrm{C}$ & 0.4633443878 & 2.9312875944 & $0.722 \varepsilon$ \\
\hline $\mathrm{H}$ & 0.3440448102 & 1.6525565402 & 4.0 \\
\hline $\mathrm{H}$ & 1.3785333073 & 3.7394128749 & 54917 \\
\hline 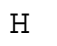 & 1.4672644082 & 4.2895060454 & 2.8 \\
\hline $\mathrm{C}$ & -0.6831923282 & 3.9723332295 & 0.68976074 \\
\hline $\mathrm{H}$ & 1.4277841222 & 3.4566695588 & 0.80746427 \\
\hline 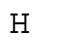 & 0.4699071302 & 2.3984126500 & -0.23115651 \\
\hline . & -0.5477341273 & 4.9264850675 & -0.51309850 \\
\hline $\mathrm{H}$ & -1.6443806984 & 3.4489335417 & 0.64453953 \\
\hline $\mathrm{H}$ & -0.6835915143 & 4.5498120528 & 1.6221507 \\
\hline 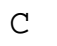 & -1.6091305293 & 5.9939461915 & -0.52371505 \\
\hline 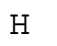 & 0.4470656418 & 5.3995531407 & -0.48080196 \\
\hline $\mathrm{H}$ & -0.5932783575 & 4.3518629214 & -1.44811531 \\
\hline $\mathrm{C}$ & -2.5211776172 & 6.1587604696 & -1.48310717 \\
\hline $\mathrm{H}$ & -1.6200254172 & 6.6615343094 & 0.33991608 \\
\hline $\mathrm{H}$ & -3.2728005208 & 6.9421970036 & -1.43162311 \\
\hline & -2.5511403023 & 5.5118709676 & -2.35819958 \\
\hline
\end{tabular}

58

$\begin{array}{lr}\mathrm{N} & -1.1308387062 \\ \mathrm{C} & -0.0896666314 \\ \mathrm{~N} & 0.7243123207 \\ \mathrm{C} & 0.1707079976 \\ \mathrm{C} & -0.9484510011 \\ \mathrm{Ru} & 0.1514898787 \\ \mathrm{P} & 0.0763213517 \\ \mathrm{C} & 1.5971839579 \\ \mathrm{C} & 1.9662163129 \\ \mathrm{C} & -2.0922080696 \\ \mathrm{C} & 1.1794563355 \\ \mathrm{C} & 2.5168890436 \\ \mathrm{C} & 3.3555819616\end{array}$

\footnotetext{
$-0.5443164322$

0.0963527361

0.5286362137

0.2862740422

$-0.7182634125$

0.1531361450

$-0.3529655829$

$-0.9960202856$

1.2626870338

$-1.3847793454$

1. 6974481501

1. 6180342571

2. 6540803367
}

-0.6502659724
-2.0551874418
-2.1429393583
-0.9599710735
-0.0685088295
-0.6701608849
1.7870732445
1.8584257772
3.2290610000
3.6013196748
-0.0945088511
2.6343659781
1.6670651511
-2.7190283736
-2.2779646225
-2.0704554750
-3.0562622553
-0.5572824403
0.9808799758
-0.2893705681
-1.5748392953
-0.3738859180
0.1184965593
0.7228491460
4.0136198316
4.6465491787
2.8851128749
0.6897607454
0.8074642700
-0.2311565198
-0.5130985093
0.6445395304
1.6221507891
-0.5237150577
-0.4808019613
-1.4481153100
-1.4831071716
0.3399160826
-1.4316231106
-2.3581995896

$-0.7331798647$

$-0.1539834930$

$-1.1388402638$

$-2.4796277947$

$-2.1812359598$

1. 9323866347

4.3169910693

5.1412451583

$-1.0000574397$

$-0.0372674136$

2.1078087400

2.7126078893

2. 9175518061 


$\begin{array}{lr}\mathrm{Cl} & 1.8621763762 \\ \mathrm{Cl} & -2.0751299372 \\ \mathrm{C} & -0.5157933228 \\ \mathrm{C} & -1.1463029341 \\ \mathrm{C} & 0.6731779988 \\ \mathrm{H} & -0.2135818319 \\ \mathrm{H} & 0.9401572388 \\ \mathrm{H} & -0.6467869371 \\ \mathrm{H} & -1.8772274770 \\ \mathrm{H} & 1.8232230199 \\ \mathrm{H} & 2.3758708474 \\ \mathrm{H} & 2.6842534202 \\ \mathrm{H} & -3.0432021436 \\ \mathrm{H} & -1.7391059792 \\ \mathrm{H} & -2.2680473079 \\ \mathrm{H} & -0.6597012239 \\ \mathrm{H} & 0.2145279529 \\ \mathrm{H} & -1.4594839835 \\ \mathrm{H} & -1.2305525366 \\ \mathrm{H} & -2.1214818169 \\ \mathrm{H} & -0.8321716055 \\ \mathrm{H} & 1.3732260858 \\ \mathrm{H} & 2.0321437354 \\ \mathrm{H} & 2.3288421940 \\ \mathrm{H} & 2.8531958734 \\ \mathrm{H} & 4.3400382262 \\ \mathrm{H} & 3.0998314544 \\ \mathrm{C} & 0.1280870215 \\ \mathrm{H} & 1.4819554034 \\ \mathrm{H} & -0.1335903372 \\ \mathrm{C} & -0.4218298435 \\ \mathrm{H} & -0.676544471 \\ \mathrm{H} & 0.9119154205 \\ \mathrm{C} & -1.0484223789 \\ \mathrm{H} & 0.3779754442 \\ \mathrm{H} & -1.1768622000 \\ \mathrm{C} & -0.6382947957 \\ \mathrm{H} & -1.8970383995 \\ \mathrm{H} & -1.1293065371 \\ \mathrm{H} & 0.2049011550 \\ & \end{array}$

$$
\begin{array}{r}
-1.5834554022 \\
1.2083099163 \\
0.9607267271 \\
-1.7118445013 \\
3.0497976438 \\
1.2235077955 \\
-0.1091688278 \\
-1.7530708466 \\
-0.5053068146 \\
2.3397242617 \\
1.0937532236 \\
0.8898049170 \\
-1.3655415443 \\
-2.4245284573 \\
-0.9830029027 \\
0.5718756524 \\
1.7756916778 \\
1.3560739613 \\
-1.9744252121 \\
-1.3819899011 \\
-2.5961143936 \\
-1.3927952415 \\
-1.7777569505 \\
-0.1876005160 \\
0.6185471785 \\
2.4938932683 \\
3.6803724412 \\
3.9111717476 \\
3.5980935747 \\
2.9044774482 \\
5.2644548821 \\
3.3521815336 \\
4.0848866745 \\
6.0604714140 \\
5.8512957144 \\
5.0664491567 \\
7.2599635860 \\
5.5863186861 \\
7.7823567438 \\
7.7680001891
\end{array}
$$

1.6312318863

2.2470575436

5.4691125019

4.5820694183

1. 6458174355

$-2.9078110946$

$-3.1505072585$

$-2.4013649997$

$-2.7198115755$

$-1.1658397212$

$-0.0064326403$

$-1.7392845245$

$-0.5803054794$

0.0228149319

0.9610830983

6.4837428175

5.4979388694

5.0831805863

5.6428816403

4. 2109806594

4. 0180456757

6.1379075004

4. 5123026700

5.2386680822

2.9716851639

3. 3503166448

2. 6712155093

2. 8113490439

1.1383414399

0.9237110295

2.3204960198

3. 2985280526

3. 5586162121

3. 4337464775

1. 8474800398

1.5447372076

3. 8506211201

3.9303295822

4. 6679567308

3.3851931595

59

C $\quad 0.3643955362$

$\mathrm{N} \quad 0.8318527603$

C $\quad-0.1716665920$

$\mathrm{N} \quad-1.3273055324$

C $\quad-1.1628265806$

C $\quad 2.2596589895$

$\mathrm{Ru} \quad-0.0656507838$

1.0592065421

$-2.6682708240$

Cl -1.7368579636

C $\quad 1.4231214380$

C $\quad 2.6303737987$

C $\quad 2.4606129157$

C $\quad 1.4143452172$

C $\quad 0.0259874416$
$-0.5421308826$

$-0.2801811093$

$-0.3005294247$

$-0.5179665827$

$-0.5189298982$

$-0.3684142941$

$-0.1006328062$

$-2.2609342365$

$-0.6161318245$

1. 7110783282

1.0334257580

0.7314696454

1.1615770070

0.3112206129

0.4504195063
2.1076459456

0.7375490873

$-0.1698517394$

0.4920684902

1. 9503281398

0.4710280579

$-2.2146307899$

$-2.3100584583$

$-0.0569395901$

$-2.2025392770$

$-2.2107669760$

$-3.0717000371$

$-4.5483908768$

$-5.2832474126$

$-4.7255646497$ 


$$
\begin{array}{r}
-0.8005048724 \\
1.4060815384 \\
2.3560208180 \\
0.7343598920 \\
0.7371972725 \\
-1.6557101817 \\
-1.6164930385 \\
2.4196970617 \\
2.6893487165 \\
2.7648432807 \\
-3.1500151328 \\
-2.6273164467 \\
-3.2673236782 \\
-1.8529350745 \\
-0.4803870913 \\
0.5107977710 \\
2.2260672007 \\
3.2702009996 \\
3.5181372882 \\
2.8176659790 \\
1.3854022989 \\
1.7152937631 \\
3.4290336909 \\
2.1855657228 \\
-0.3781795003
\end{array}
$$

60

$\mathrm{N}$

C

$\mathrm{N}$

C

C

$\mathrm{Ru}$

$\mathrm{Cl}$

C

C

C

C

C

C

C

C

C

$\mathrm{Cl}$

$\mathrm{H}$

$\mathrm{H}$

$\mathrm{H}$

$\mathrm{H}$

$\mathrm{H}$

$\mathrm{H}$

$\mathrm{H}$

$\mathrm{H}$

$\mathrm{H}$

$\mathrm{H}$

$\mathrm{H}$

$\mathrm{H}$
0.7998214371 $-0.1435687932$ $-1.1010446107$ $-0.9102628862$ 0.5687771350 $-0.2091447971$ $-1.7363878894$ $-2.3912890805$

2. 1134152018 1.1089950022 1.0899509886 2.1742249105 2. 3442045235

2.0554758252

0.6510959816 $-0.2903519005$ $-1.3830296431$ 0.8143026211

$$
0.7745910148
$$
1. 2233124408 $-1.1284605327$ $-1.5718844674$ 2.0294506320

2.8240146333

2.4870089383 $-2.8193040653$ $-2.2435893105$ $-3.0785709159$ $-2.3358094091$ $-1.4210802264$

$$
\begin{array}{r}
-0.5945954384 \\
2.2618817188 \\
3.2189128133 \\
0.2214459686 \\
-1.5190662483 \\
-1.3897326631 \\
0.3865621204 \\
-0.6604939895 \\
-1.1465820152 \\
0.5815852076 \\
-1.5204242469 \\
-0.6684886841 \\
0.2616644766 \\
-0.4142369628 \\
-1.6218224761 \\
2.4257266370 \\
4.1255310568 \\
3.1523240310 \\
1.2299227437 \\
-0.3474400637 \\
0.6314008608 \\
-0.7429679060 \\
1.0585034613 \\
2.2238409369 \\
1.4590778925
\end{array}
$$

$-4.4423205646$

$-1.4102513668$

$-1.4122093754$

2. 8006375805

2.4448555761

2.3968089469

2.3768033621

$-0.5644555906$

1. 1135737350

0.6823209852

0.3361234403

$-1.1426391884$

0.2113440039

$-4.2345115063$

$-4.5914069047$

$-0.8199993017$

$-0.8267879189$

$-1.9947222132$

$-2.6618546523$

$-3.0460116640$

$-6.3368556996$

$-5.2725240805$

$-5.0537664264$

$-4.5976581088$

$-4.6545941611$
0.0860574344

$-0.3571581073$

$-0.9935666435$

$-0.8294007468$

$-0.4235482253$

$-0.1286847153$

1. 7677087448

$-1.3758627942$

0.5709291784

0.8117744624

2. 2913641862

3.0799315787

0.1549363396

$-0.0235455419$

$-0.6328487325$

0.1400661989

$-0.5658281550$

$-2.3719313961$

0.3494110762

$-1.2860485849$

$-1.7601298099$

$-0.0378763853$

1. 1246831517

$-0.2562737669$

1. 2494128120

$-2.1718317412$

$-1.7710712366$

$-0.5230815559$

$-0.0507034208$

$-1.6455894368$
0.6430760521

$-0.2040491286$

0.4892482148

1. 9387959486

2.0057745926

$-2.2417486472$

$-1.8073235259$

$-0.0667177011$

0.2518875351

$-3.2959266848$

$-3.2675953557$

$-3.1699133535$

$-3.8991680056$

$-5.4036275714$

$-5.4814118073$

$-4.5653058184$

$-3.9174010355$

$-2.3347900226$

2.7519244336

2.1985649456

2.4704538105

2.3191888136

$-0.6840303443$

0.1223089725

1. 0254930172

0.5501842463

$-1.0755112522$

$-0.1094254806$

$-3.8234680694$

$-4.0556696971$ 


$\begin{array}{lr}\mathrm{H} & 0.1092256356 \\ \mathrm{H} & 2.0598580938 \\ \mathrm{H} & 3.1921119285 \\ \mathrm{H} & 3.2272322984 \\ \mathrm{H} & 2.5224460130 \\ \mathrm{H} & 0.2509425354 \\ \mathrm{H} & 0.6809234171 \\ \mathrm{H} & 2.8069015513 \\ \mathrm{H} & 2.0760873997 \\ \mathrm{H} & -0.5558844587\end{array}$

61

\begin{tabular}{|c|c|}
\hline $\mathrm{N}$ & -0.9926488333 \\
\hline $\mathrm{C}$ & -0.0748743946 \\
\hline $\mathrm{N}$ & 0.7946205366 \\
\hline $\mathrm{C}$ & 0.5622902397 \\
\hline $\mathrm{C}$ & -0.8662753018 \\
\hline $\mathrm{Ru}$ & -0.0013760492 \\
\hline $\mathrm{Cl}$ & -1.5666481856 \\
\hline $\mathrm{C}$ & 2.0491616974 \\
\hline $\mathrm{C}$ & -2.2525055475 \\
\hline $\mathrm{C}$ & 0.9841311180 \\
\hline $\mathrm{C}$ & 0.1945027251 \\
\hline $\mathrm{C}$ & -1.0664927058 \\
\hline $\mathrm{C}$ & -1.0278677944 \\
\hline $\mathrm{C}$ & -2.1113534381 \\
\hline $\mathrm{Cl}$ & 1.8149045557 \\
\hline $\mathrm{H}$ & 0.6573763838 \\
\hline $\mathrm{H}$ & 1.2920126737 \\
\hline $\mathrm{H}$ & -1.0115458675 \\
\hline $\mathrm{H}$ & -1.6168063059 \\
\hline $\mathrm{H}$ & 1.9215471659 \\
\hline $\mathrm{H}$ & 2.8587612690 \\
\hline $\mathrm{H}$ & 2.3045981078 \\
\hline $\mathrm{H}$ & -2.6274980455 \\
\hline $\mathrm{H}$ & -2.0866249881 \\
\hline $\mathrm{H}$ & -2.9927142554 \\
\hline $\mathrm{H}$ & 2.0606697735 \\
\hline $\mathrm{H}$ & 0.6780600819 \\
\hline $\mathrm{C}$ & -0.5115897185 \\
\hline $\mathrm{C}$ & -2.3467734578 \\
\hline $\mathrm{H}$ & 0.8962232830 \\
\hline $\mathrm{H}$ & -0.0402403413 \\
\hline $\mathrm{H}$ & -2.0034989246 \\
\hline $\mathrm{H}$ & -3.1263241787 \\
\hline $\mathrm{H}$ & -2.8523552475 \\
\hline $\mathrm{C}$ & -1.8929081749 \\
\hline $\mathrm{H}$ & -3.0361617081 \\
\hline $\mathrm{H}$ & -0.6166595204 \\
\hline $\mathrm{H}$ & 0.0846942161 \\
\hline $\mathrm{H}$ & -1.8136962615 \\
\hline $\mathrm{H}$ & -2.5893494820 \\
\hline
\end{tabular}

2. 7547712686

4.1580876245

2. 7015278271

0.7839931168

$-0.8167293018$

$-0.6070203092$

$-1.6775571611$

$-0.6595054645$

0.9540712742

1.1439393534
$-3.2414028493$

$-3.0966656123$

$-3.1349968088$

$-3.7439670689$

$-3.4385034028$

$-6.5036469467$

$-5.1558473083$

$-5.8854314888$

$-5.9037130781$

$-4.8962629239$
$-0.9764962308$
$-0.5396702403$
$-0.2603566738$
0.1311419536
0.2864531114
$-0.7272124593$
$-0.1985946598$
$-2.0969959005$
$-0.7534499893$
$-1.9901902788$
$-0.0855783053$
2.1482731348
1. 7656678322
0.9215997862
1.6291706335
$-1.4343313795$
1.0483363044
0.1220866249
$-0.6687658012$
$-2.1459325585$
$-2.9423664924$
$-1.7172478050$
0.6161539767
$-0.9823240124$
$-0.3528944496$
0.0310087960
3. 4413634011
4.4383458432
3.7839929511
3. 8627131447
3. 8587712895
2.1952404209
$-2.8206657402$
$-1.6855351404$
$-2.3458785435$
$-0.0211326639$
$-2.5435082361$
1. 4196165236
0.1868839623
$-2.3207723245$
0.6097289340
$-0.2684876270$
1. 6734270739
$-1.1063169552$
$-2.2680701357$
$-1.7947029748$
$-0.5704921931$
$-0.6252473624$
1. 0484601404
0.8815344904
2.0948471839
0.0530354967
3. 4816266034
3. 4685520512
0.9636541213
5.5348485594
0.0361279356
4.2631560913
$-0.6206582543$
$-2.5982313721$
4.8207756357
3.8655953504
$-4.0231152690$
$-2.5549421037$
3. 8823164694
3.8275856359
$-0.6256364982$
1. 3224526694
0.2366992064
4. 9784552625
4.9727042764
3.4399340782
0.3238244463
1.8349962519
6.4210361494
5.8239615122
2.1366637900
4.2486095799
1.6316185276
5.7602386893 


\begin{tabular}{|c|c|c|c|}
\hline $\mathrm{C}$ & -1.0191683579 & -0.5870892696 & 4.068755247 \\
\hline $\mathrm{C}$ & 0.2084195543 & -0.0354450211 & 4.548529398 \\
\hline $\mathrm{C}$ & -0.1038526752 & 1.0980191046 & 5.519850786 \\
\hline C & -1.5334951149 & 1.5350604476 & 5.13867230 \\
\hline C & -2.1873523907 & 0.2567294521 & 4.57449760 \\
\hline & 0.0279687569 & -0.0310970640 & 2.15610800 \\
\hline+ & -1.6357342251 & 1.7355959642 & 1.704230444 \\
\hline $\mathrm{C}$ & -1.1917610628 & -2.0063246036 & 3.72246754 \\
\hline $\mathrm{C}$ & -2.3826151627 & -2.5675898437 & 3.4411511 \\
\hline C & 0.1362632667 & -0.1522140736 & 0.102945374 \\
\hline $\mathrm{N}$ & -0.8217954722 & -0.7189554159 & -0.6565 \\
\hline C & -0.5644031998 & -0.5131430854 & -2.090272891 \\
\hline $\mathrm{C}$ & 0.9251273495 & -0.1540198058 & -2.088270112 \\
\hline $\mathrm{N}$ & 1.1301986062 & 0.2762156162 & $-0.69507007 \varepsilon$ \\
\hline C & -2.1706135396 & -1.0455187923 & -0.215882051 \\
\hline 0 & 2.4626324994 & 0.6698260078 & -0.266118745 \\
\hline & 1.7815254242 & -1.7650688276 & 2.249534717 \\
\hline c & 1.1772218878 & 1.1599298762 & 2.973301323 \\
\hline $\mathrm{H}$ & 1.1797402431 & 0.6487292993 & -2.786605917 \\
\hline $\mathrm{H}$ & 1.5601324851 & -1.0251728043 & -2.30566450 \\
\hline $\mathrm{H}$ & -0.7938836135 & -1.4161262970 & -2.663862456 \\
\hline & -1.1883259692 & 0.3113850508 & -2.465057233 \\
\hline $\mathrm{H}$ & 2.3958042801 & 1.1681956692 & 0.699387911 \\
\hline $\mathrm{H}$ & 3.1205828599 & -0.2017324716 & -0.166834466 \\
\hline $\mathrm{H}$ & 2.8761338425 & 1.3667984211 & -1.002470695 \\
\hline & -2.5498098668 & -1.8661636991 & -0.834373168 \\
\hline $\mathrm{H}$ & -2.1540255734 & -1.3790677117 & 0.82267295 \\
\hline $\mathrm{H}$ & -2.8348862494 & -0.1774500704 & -0.294237105 \\
\hline $\mathrm{H}$ & 2.2104352524 & 0.8965241350 & 3.22113520 \\
\hline $\mathrm{H}$ & 0.8665469521 & 2.1891335746 & $3.17726371^{\prime}$ \\
\hline $\mathrm{H}$ & 1.0778392656 & -0.6716956493 & 4.685158135 \\
\hline $\mathrm{H}$ & -0.2889036189 & -2.6092298836 & 3.686839568 \\
\hline $\mathrm{H}$ & -2.4552603823 & -3.6247291165 & 3.202459238 \\
\hline $\mathrm{H}$ & -3.3094411003 & -2.0001692744 & 3.448468358 \\
\hline & -0.0845188031 & 0.6549600499 & $6.52810221 \varepsilon$ \\
\hline $\mathrm{H}$ & 0.6227600262 & 1.9159791196 & 5.521374060 \\
\hline & -2.6820595126 & -0.3204338361 & 5.370680855 \\
\hline & -2.9288563239 & 0.4744884093 & 3.80252398 \\
\hline & -1.4985944640 & 2.2941299905 & $4.35331061 \varepsilon$ \\
\hline & -2.0833675274 & 1.9471066333 & 5.99079870 \\
\hline
\end{tabular}

63

$\begin{array}{lrrr}\mathrm{N} & -0.9342194070 & -0.8647623445 & -0.6020192660 \\ \mathrm{C} & 0.0013205684 & -0.2098003832 & 0.1199951486 \\ \mathrm{~N} & 0.9289731922 & 0.2751143515 & -0.7483270114 \\ \mathrm{C} & 0.6961366691 & -0.2046010451 & -2.1217225506 \\ \mathrm{C} & -0.7497037696 & -0.6849997671 & -2.0497000203 \\ \mathrm{Ru} & 0.0012822020 & -0.0494213014 & 2.1743653061 \\ \mathrm{Cl} & -2.1288366572 & 1.1734131509 & 1.9139940449 \\ \mathrm{C} & 2.2961618278 & 0.6666382513 & -0.4368107183 \\ \mathrm{C} & -2.2043370352 & -1.4103974490 & -0.1500978151 \\ \mathrm{C} & 0.8202648944 & 1.5798606226 & 2.1873252179 \\ \mathrm{C} & 0.4663078398 & -0.0739885482 & 4.7383889324 \\ \mathrm{C} & -0.7537941647 & -0.6502246947 & 4.4899690516 \\ \mathrm{C} & -0.9593501391 & -1.9810439107 & 3.9148741226\end{array}$




$\begin{array}{lrrr}\mathrm{C} & -2.1514140259 & -2.4449106101 & 3.4946049136 \\ \mathrm{Cl} & 1.9265117857 & -1.5763790590 & 2.1593233884 \\ \mathrm{H} & 0.8559593399 & 0.5967995371 & -2.8500907841 \\ \mathrm{H} & 1.3912945051 & -1.0262748417 & -2.3504622747 \\ \mathrm{H} & -0.9238967578 & -1.6227593105 & -2.5864135173 \\ \mathrm{H} & -1.4571754017 & 0.0690883847 & -2.4248431850 \\ \mathrm{H} & 2.3311751187 & 1.1822858170 & 0.5240662484 \\ \mathrm{H} & 2.9508469756 & -0.2110345711 & -0.3771011398 \\ \mathrm{H} & 2.6501850578 & 1.3422449600 & -1.2228501469 \\ \mathrm{H} & -2.3895000068 & -2.3511215788 & -0.6829973625 \\ \mathrm{H} & -2.1684735533 & -1.6062752322 & 0.9165181397 \\ \mathrm{H} & -3.0238927540 & -0.7095904641 & -0.3439723413 \\ \mathrm{H} & 1.6992083457 & 1.7746163175 & 2.8162779236 \\ \mathrm{H} & 0.4495627463 & 2.4380309002 & 1.6157678705 \\ \mathrm{C} & 0.3174722575 & 1.1994952374 & 5.5459631989 \\ \mathrm{C} & -1.8687026481 & 0.1694713367 & 5.1173690046 \\ \mathrm{H} & 1.4161439281 & -0.5707028205 & 4.5680939888 \\ \mathrm{H} & -0.0755850708 & -2.6037815616 & 3.8091798124 \\ \mathrm{H} & -2.2439056629 & -3.4437803834 & 3.0770364391 \\ \mathrm{H} & -3.0579550227 & -1.8486455445 & 3.5582354525 \\ \mathrm{H} & -2.2060473896 & -0.3511193227 & 6.0268447152 \\ \mathrm{H} & -2.7339238992 & 0.2983622457 & 4.4635301146 \\ \mathrm{H} & 0.6133645489 & 0.9885044207 & 6.5865022887 \\ \mathrm{H} & 0.9595240841 & 2.0196383689 & 5.2001004397 \\ \mathrm{C} & -1.1936327861 & 1.5221441244 & 5.4409393762 \\ \mathrm{H} & -1.5895972914 & 1.9963189366 & 6.3452428394 \\ \mathrm{H} & -1.3652284903 & 2.1999651900 & 4.5992343135\end{array}$




\section{Basis sets and ECP}

\section{Effective Core Potential}

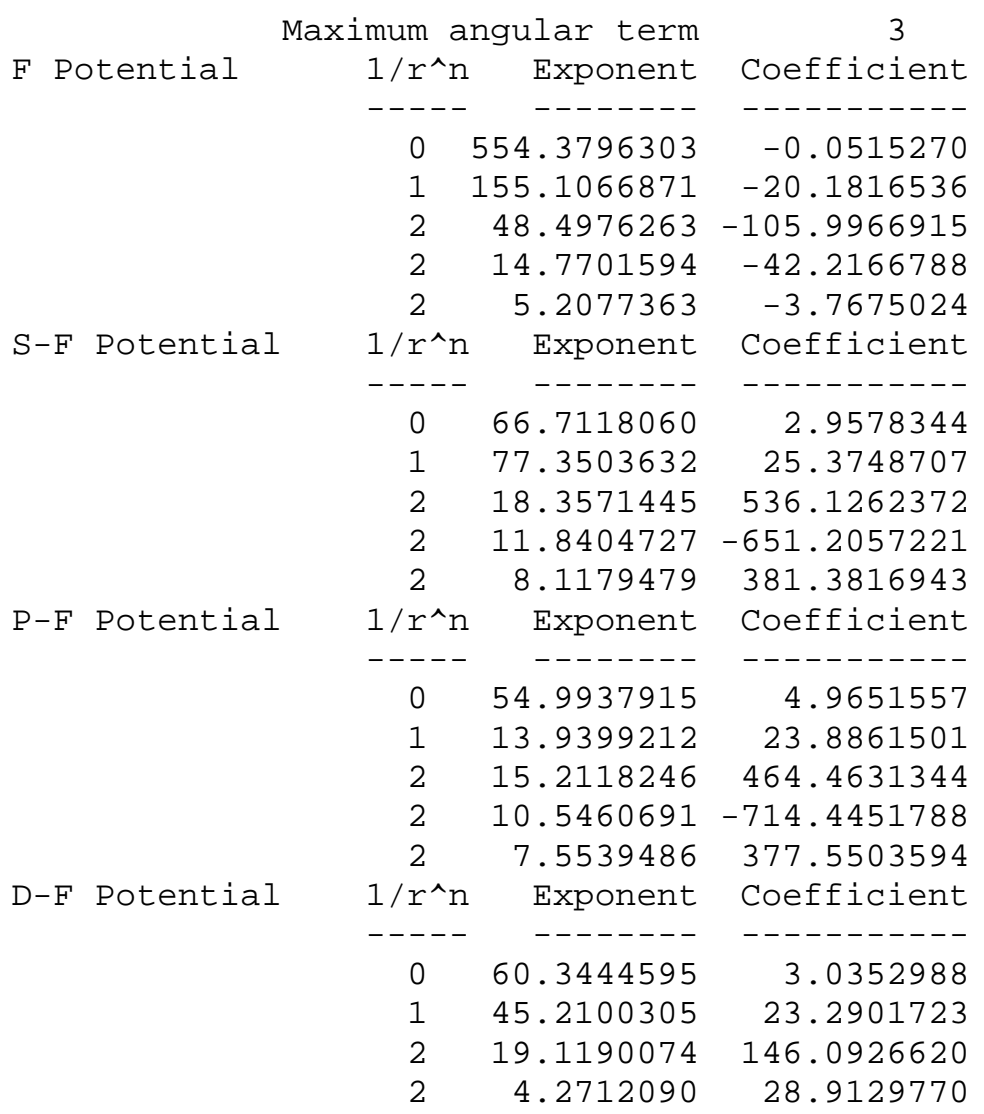

\section{Basis Set for Geometry Optimization (LACVP*)}

$\begin{array}{lccc} & \mathrm{S} & & \\ & \mathrm{h} & \mathrm{t} & \\ & \mathrm{e} & \mathrm{Y} & \\ & \mathrm{l} & \mathrm{P} & \mathrm{f} \\ \text { atom } & 1 & \mathrm{e} & \mathrm{n} \\ ---- & --- & --- & --- \\ \mathrm{C} & 1 & \mathrm{~S} & 1 \\ \mathrm{C} & 2 & \mathrm{~S} & 1 \\ \mathrm{C} & 3 & \mathrm{~S} & 1 \\ \mathrm{C} & 4 & \mathrm{~S} & 1 \\ \mathrm{C} & 5 & \mathrm{~S} & 1 \\ \mathrm{C} & 6 & \mathrm{~S} & 1 \\ \mathrm{C} & 7 & \mathrm{~S} & 2 \\ \mathrm{C} & 8 & \mathrm{~S} & 2 \\ \mathrm{C} & 9 & \mathrm{~S} & 2 \\ \mathrm{C} & 10 & \mathrm{X} & 3 \\ & & \mathrm{Y} & 4 \\ & & \mathrm{Z} & 5 \\ \mathrm{C} & 11 & \mathrm{X} & 3 \\ & & \mathrm{Y} & 4 \\ & & \mathrm{Z} & 5\end{array}$

$$
\begin{array}{r}
z \\
------- \\
3047.524880 \\
457.369518 \\
103.948685 \\
29.210155 \\
9.286663 \\
3.163927 \\
7.868272 \\
1.881289 \\
0.544249 \\
7.868272 \\
\\
1.881289
\end{array}
$$

\begin{tabular}{r}
\multicolumn{1}{l}{ rcoef } \\
-0.536345 \\
0.989452 \\
1.597283 \\
2.079187 \\
1.774174 \\
0.612580 \\
-0.399556 \\
-0.184155 \\
0.516390 \\
1.296082 \\
\end{tabular}

\begin{aligned} \multicolumn{1}{c}{ rmfac } \\ 1.000000 \\ 1.000000 \\ 1.000000 \\ 1.000000 \\ 1.000000 \\ 1.000000 \\ 1.000000 \\ 1.000000 \\ 1.000000 \\ 1.000000 \\ 1.000000 \\ 1.000000 \\ 1.000000 \\ 1.000000 \\ 1.000000\end{aligned}

rcoef*rmfac
0.536345
0.989452
1.597283
2.079187
1.774174
0.612580
-0.399556
-0.184155
0.516390
1.296082
1.296082
1.296082
0.993754
0.993754
0.993754




\begin{tabular}{|c|c|c|c|c|c|c|c|}
\hline \multirow[t]{3}{*}{ C } & \multirow[t]{3}{*}{12} & $\mathrm{X}$ & 3 & \multirow[t]{3}{*}{0.544249} & \multirow[t]{3}{*}{0.495953} & 1.000000 & 0.495953 \\
\hline & & Y & 4 & & & 1.000000 & 0.495953 \\
\hline & & Z & 5 & & & 1.000000 & 0.495953 \\
\hline C & 13 & $S$ & 6 & 0.168714 & 0.187618 & 1.000000 & 0.187618 \\
\hline \multirow[t]{3}{*}{ C } & \multirow[t]{3}{*}{14} & $\mathrm{x}$ & 7 & \multirow[t]{3}{*}{0.168714} & \multirow[t]{3}{*}{0.154128} & 1.000000 & 0.154128 \\
\hline & & Y & 8 & & & 1.000000 & 0.154128 \\
\hline & & Z & 9 & & & 1.000000 & 0.154128 \\
\hline \multirow[t]{6}{*}{$\mathrm{C}$} & \multirow[t]{6}{*}{15} & $x x$ & 10 & \multirow[t]{6}{*}{0.800000} & \multirow[t]{6}{*}{1.113825} & 1.000000 & 1.113825 \\
\hline & & $Y Y$ & 11 & & & 1.000000 & 1.113825 \\
\hline & & $\mathrm{ZZ}$ & 12 & & & 1.000000 & 1.113825 \\
\hline & & $X Y$ & 13 & & & 1.732051 & 1.929201 \\
\hline & & $\mathrm{XZ}$ & 14 & & & 1.732051 & 1.929201 \\
\hline & & $\mathrm{YZ}$ & 15 & & & 1.732051 & 1.929201 \\
\hline $\mathrm{N}$ & 1 & $S$ & 16 & 4173.511460 & 0.678998 & 1.000000 & 0.678998 \\
\hline $\mathrm{N}$ & 2 & $S$ & 16 & 627.457911 & 1.250431 & 1.000000 & 1.250431 \\
\hline $\mathrm{N}$ & 3 & $S$ & 16 & 142.902093 & 2.020356 & 1.000000 & 2.020356 \\
\hline $\mathrm{N}$ & 4 & $S$ & 16 & 40.234329 & 2.644212 & 1.000000 & 2.644212 \\
\hline $\mathrm{N}$ & 5 & $S$ & 16 & 12.820213 & 2.265005 & 1.000000 & 2.265005 \\
\hline $\mathrm{N}$ & 6 & $S$ & 16 & 4.390437 & 0.779188 & 1.000000 & 0.779188 \\
\hline $\mathrm{N}$ & 7 & $S$ & 17 & 11.626362 & -0.515875 & 1.000000 & -0.515875 \\
\hline $\mathrm{N}$ & 8 & $S$ & 17 & 2.716280 & -0.255023 & 1.000000 & -0.255023 \\
\hline $\mathrm{N}$ & 9 & $S$ & 17 & 0.772218 & 0.672735 & 1.000000 & 0.672735 \\
\hline \multirow[t]{3}{*}{$\mathrm{N}$} & \multirow[t]{3}{*}{10} & $\mathrm{x}$ & 18 & \multirow[t]{3}{*}{11.626362} & \multirow[t]{3}{*}{2.068052} & 1.000000 & 2.068052 \\
\hline & & $\mathrm{Y}$ & 19 & & & 1.000000 & 2.068052 \\
\hline & & Z & 20 & & & 1.000000 & 2.068052 \\
\hline \multirow[t]{3}{*}{$\mathrm{N}$} & 11 & $\mathrm{x}$ & 18 & 2.716280 & 1.610011 & 1.000000 & 1.610011 \\
\hline & & $\mathrm{Y}$ & 19 & & & 1.000000 & 1.610011 \\
\hline & & Z & 20 & & & 1.000000 & 1.610011 \\
\hline $\mathrm{N}$ & 12 & $\mathrm{X}$ & 18 & 0.772218 & 0.764490 & 1.000000 & 0.764490 \\
\hline & & Y & 19 & & & 1.000000 & 0.764490 \\
\hline & & Z & 20 & & & 1.000000 & 0.764490 \\
\hline $\mathrm{N}$ & 13 & S & 21 & 0.212031 & 0.222695 & 1.000000 & 0.222695 \\
\hline $\mathrm{N}$ & 14 & $\mathrm{X}$ & 22 & 0.212031 & 0.205088 & 1.000000 & 0.205088 \\
\hline & & Y & 23 & & & 1.000000 & 0.205088 \\
\hline & & Z & 24 & & & 1.000000 & 0.205088 \\
\hline $\mathrm{N}$ & 15 & $x X$ & 25 & 0.800000 & 1.113825 & 1.000000 & 1.113825 \\
\hline & & $Y Y$ & 26 & & & 1.000000 & 1.113825 \\
\hline & & $\mathrm{ZZ}$ & 27 & & & 1.000000 & 1.113825 \\
\hline & & $X Y$ & 28 & & & 1.732051 & 1.92920 \\
\hline & & $\mathrm{XZ}$ & 29 & & & 1.732051 & 1.929201 \\
\hline & & $\mathrm{YZ}$ & 30 & & & 1.732051 & 1.929201 \\
\hline $\mathrm{Ru}$ & 1 & S & 76 & 2.565000 & -1.504161 & 1.000000 & -1.504161 \\
\hline $\mathrm{Ru}$ & 2 & S & 76 & 1.508000 & 1.289616 & 1.000000 & 1.289616 \\
\hline Ru & 3 & S & 76 & 0.512900 & 0.242621 & 1.000000 & 0.24262 \\
\hline $\mathrm{Ru}$ & 4 & S & 77 & 1.508000 & -0.284585 & 1.000000 & -0.28458 \\
\hline $\mathrm{Ru}$ & 5 & S & 77 & 0.512900 & -0.322652 & 1.000000 & -0.32265 \\
\hline Ru & 6 & S & 77 & 0.136200 & 0.159788 & 1.000000 & 0.159788 \\
\hline $\mathrm{Ru}$ & 7 & $S$ & 78 & 0.041700 & 0.065768 & 1.000000 & 0.06576 \\
\hline $\mathrm{Ru}$ & 8 & $\mathrm{x}$ & 79 & 4.859000 & -0.972528 & 1.000000 & -0.97252 \\
\hline & & $\mathrm{Y}$ & 80 & & & 1.000000 & -0.97252 \\
\hline & & Z & 81 & & & 1.000000 & -0.972528 \\
\hline $\mathrm{Ru}$ & 9 & $\mathrm{X}$ & 79 & 1.219000 & 1.357419 & 1.000000 & 1.35741 \\
\hline & & Y & 80 & & & 1.000000 & 1.35741 \\
\hline & & Z & 81 & & & 1.000000 & 1.35741 \\
\hline $\mathrm{Ru}$ & 10 & $\mathrm{X}$ & 79 & 0.441300 & 0.188063 & 1.000000 & 0.18806 \\
\hline & & $\mathrm{Y}$ & 80 & & & 1.000000 & 0.18806 \\
\hline & & Z & 81 & & & 1.000000 & 0.18806 \\
\hline
\end{tabular}




\begin{tabular}{|c|c|c|c|c|c|c|c|}
\hline \multirow[t]{3}{*}{$\mathrm{Ru}$} & 11 & $\mathrm{x}$ & 82 & 0.083000 & 0.063502 & 1.000000 & 0.063502 \\
\hline & & $\mathrm{Y}$ & 83 & & & 1.000000 & 0.063502 \\
\hline & & Z & 84 & & & 1.000000 & 0.063502 \\
\hline \multirow[t]{3}{*}{$\mathrm{Ru}$} & 12 & $\mathrm{X}$ & 85 & 0.025000 & 0.014170 & 1.000000 & 0.014170 \\
\hline & & $\mathrm{Y}$ & 86 & & & 1.000000 & 0.014170 \\
\hline & & $\mathrm{Z}$ & 87 & & & 1.000000 & 0.014170 \\
\hline \multirow[t]{6}{*}{$\mathrm{Ru}$} & 13 & $\mathrm{xx}$ & 88 & 4.105000 & 0.946049 & 1.000000 & 0.946049 \\
\hline & & YY & 89 & & & 1.000000 & 0.946049 \\
\hline & & $\mathrm{ZZ}$ & 90 & & & 1.000000 & 0.946049 \\
\hline & & $X Y$ & 91 & & & 1.732051 & 1.638605 \\
\hline & & $\mathrm{XZ}$ & 92 & & & 1.732051 & 1.638605 \\
\hline & & $\mathrm{YZ}$ & 93 & & & 1.732051 & 1.638605 \\
\hline \multirow[t]{6}{*}{$\mathrm{Ru}$} & 14 & $\mathrm{xX}$ & 88 & 1.377000 & 1.470180 & 1.000000 & 1.470180 \\
\hline & & YY & 89 & & & 1.000000 & 1.470180 \\
\hline & & $\mathrm{ZZ}$ & 90 & & & 1.000000 & 1.470180 \\
\hline & & $X Y$ & 91 & & & 1.732051 & 2.546426 \\
\hline & & $\mathrm{xz}$ & 92 & & & 1.732051 & 2.546426 \\
\hline & & $\mathrm{YZ}$ & 93 & & & 1.732051 & 2.546426 \\
\hline \multirow[t]{6}{*}{$\mathrm{Ru}$} & 15 & $\mathrm{XX}$ & 88 & 0.482800 & 0.263615 & 1.000000 & 0.263615 \\
\hline & & YY & 89 & & & 1.000000 & 0.263615 \\
\hline & & $\mathrm{ZZ}$ & 90 & & & 1.000000 & 0.263615 \\
\hline & & $X Y$ & 91 & & & 1.732051 & 0.456595 \\
\hline & & $\mathrm{XZ}$ & 92 & & & 1.732051 & 0.456595 \\
\hline & & $\mathrm{YZ}$ & 93 & & & 1.732051 & 0.456595 \\
\hline \multirow[t]{6}{*}{$\mathrm{Ru}$} & 16 & $\mathrm{xX}$ & 94 & 0.150100 & 0.059577 & 1.000000 & 0.059577 \\
\hline & & YY & 95 & & & 1.000000 & 0.059577 \\
\hline & & $\mathrm{ZZ}$ & 96 & & & 1.000000 & 0.059577 \\
\hline & & $X Y$ & 97 & & & 1.732051 & 0.103190 \\
\hline & & XZ & 98 & & & 1.732051 & 0.103190 \\
\hline & & $\mathrm{YZ}$ & 99 & & & 1.732051 & 0.103190 \\
\hline $\mathrm{P}$ & 1 & $S$ & 100 & 19413.300000 & 2.170359 & 1.000000 & 2.170359 \\
\hline $\mathrm{P}$ & 2 & $S$ & 100 & 2909.420000 & 4.010907 & 1.000000 & 4.010907 \\
\hline $\mathrm{P}$ & 3 & $\mathrm{~S}$ & 100 & 661.364000 & 6.506317 & 1.000000 & 6.506317 \\
\hline $\mathrm{P}$ & 4 & $\mathrm{~S}$ & 100 & 185.759000 & 8.609470 & 1.000000 & 8.609470 \\
\hline $\mathrm{P}$ & 5 & $\mathrm{~S}$ & 100 & 59.194300 & 7.373069 & 1.000000 & 7.373069 \\
\hline$P$ & 6 & $S$ & 100 & 20.031000 & 2.261991 & 1.000000 & 2.261991 \\
\hline $\mathrm{P}$ & 7 & $\mathrm{~S}$ & 101 & 339.478000 & -0.156820 & 1.000000 & -0.156820 \\
\hline $\mathrm{P}$ & 8 & $\mathrm{~S}$ & 101 & 81.010100 & -0.693775 & 1.000000 & -0.693775 \\
\hline $\mathrm{P}$ & 9 & $S$ & 101 & 25.878000 & -0.953723 & 1.000000 & -0.953723 \\
\hline $\mathrm{P}$ & 10 & $\mathrm{~S}$ & 101 & 9.452210 & 0.372034 & 1.000000 & 0.372034 \\
\hline $\mathrm{P}$ & 11 & $S$ & 101 & 3.665660 & 1.160081 & 1.000000 & 1.160081 \\
\hline $\mathrm{P}$ & 12 & $\mathrm{~S}$ & 101 & 1.467460 & 0.383706 & 1.000000 & 0.383706 \\
\hline \multirow[t]{3}{*}{$\mathrm{P}$} & 13 & $\mathrm{X}$ & 102 & 339.478000 & 9.481104 & 1.000000 & 9.481104 \\
\hline & & $\mathrm{Y}$ & 103 & & & 1.000000 & 9.481104 \\
\hline & & $\mathrm{Z}$ & 104 & & & 1.000000 & 9.481104 \\
\hline \multirow[t]{3}{*}{$\mathrm{P}$} & 14 & $\mathrm{X}$ & 102 & 81.010100 & 11.672425 & 1.000000 & 11.672425 \\
\hline & & $\mathrm{Y}$ & 103 & & & 1.000000 & 11.672425 \\
\hline & & $\mathrm{Z}$ & 104 & & & 1.000000 & 11.672425 \\
\hline \multirow[t]{3}{*}{$\mathrm{P}$} & 15 & $\mathrm{X}$ & 102 & 25.878000 & 11.627075 & 1.000000 & 11.627075 \\
\hline & & $\mathrm{Y}$ & 103 & & & 1.000000 & 11.627075 \\
\hline & & $\mathrm{Z}$ & 104 & & & 1.000000 & 11.627075 \\
\hline \multirow[t]{3}{*}{$\mathrm{P}$} & 16 & $\mathrm{x}$ & 102 & 9.452210 & 8.017143 & 1.000000 & 8.017143 \\
\hline & & $\mathrm{Y}$ & 103 & & & 1.000000 & 8.017143 \\
\hline & & $\mathrm{z}$ & 104 & & & 1.000000 & 8.017143 \\
\hline \multirow[t]{3}{*}{$\mathrm{P}$} & 17 & $\mathrm{x}$ & 102 & 3.665660 & 3.260096 & 1.000000 & 3.260096 \\
\hline & & $\mathrm{Y}$ & 103 & & & 1.000000 & 3.260096 \\
\hline & & $\mathrm{Z}$ & 104 & & & 1.000000 & 3.260096 \\
\hline$r$ & 18 & $\mathrm{x}$ & 102 & 1.467460 & 0.549278 & 1.000000 & 0.549278 \\
\hline
\end{tabular}




\begin{tabular}{|c|c|c|c|c|c|c|c|}
\hline & & $\mathrm{Y}$ & 103 & & & 1.000000 & 0.549278 \\
\hline & & z & 104 & & & 1.000000 & 0.549278 \\
\hline $\mathrm{P}$ & 19 & S & 105 & 2.156230 & -1.414076 & 1.000000 & -1.414076 \\
\hline P & 20 & $\mathrm{~S}$ & 105 & 0.748997 & 0.083106 & 1.000000 & 0.083106 \\
\hline \multirow[t]{3}{*}{ P } & 21 & $\mathrm{x}$ & 106 & 2.156230 & -0.252893 & 1.000000 & -0.252893 \\
\hline & & $\mathrm{Y}$ & 107 & & & 1.000000 & -0.252893 \\
\hline & & z & 108 & & & 1.000000 & -0.252893 \\
\hline \multirow[t]{3}{*}{ P } & 22 & $\mathrm{x}$ & 106 & 0.748997 & 1.040371 & 1.000000 & 1.040371 \\
\hline & & $\mathrm{Y}$ & 107 & & & 1.000000 & 1.040371 \\
\hline & & $\mathrm{z}$ & 108 & & & 1.000000 & 1.040371 \\
\hline $\mathrm{P}$ & 23 & $\mathrm{~S}$ & 105 & 0.283145 & 0.276641 & 1.000000 & 0.276641 \\
\hline \multirow[t]{3}{*}{$\mathrm{P}$} & 24 & $\mathrm{x}$ & 106 & 0.283145 & 0.294409 & 1.000000 & 0.294409 \\
\hline & & $\mathrm{Y}$ & 107 & & & 1.000000 & 0.294409 \\
\hline & & z & 108 & & & 1.000000 & 0.294409 \\
\hline P & 25 & $\mathrm{~S}$ & 109 & 0.099832 & 0.126579 & 1.000000 & 0.126579 \\
\hline \multirow[t]{3}{*}{$\mathrm{P}$} & 26 & $\mathrm{x}$ & 110 & 0.099832 & 0.079988 & 1.000000 & 0.079988 \\
\hline & & $\mathrm{Y}$ & 111 & & & 1.000000 & 0.079988 \\
\hline & & Z & 112 & & & 1.000000 & 0.079988 \\
\hline \multirow[t]{6}{*}{$\mathrm{P}$} & 27 & $\mathrm{XX}$ & 113 & 0.550000 & 0.578155 & 1.000000 & 0.578155 \\
\hline & & YY & 114 & & & 1.000000 & 0.578155 \\
\hline & & $\mathrm{zZ}$ & 115 & & & 1.000000 & 0.578155 \\
\hline & & $X Y$ & 116 & & & 1.732051 & 1.001394 \\
\hline & & $\mathrm{XZ}$ & 117 & & & 1.732051 & 1.001394 \\
\hline & & $\mathrm{YZ}$ & 118 & & & 1.732051 & 1.001394 \\
\hline $\mathrm{Cl}$ & 1 & $\mathrm{~S}$ & 179 & 25180.100000 & 2.611296 & 1.000000 & 2.611296 \\
\hline $\mathrm{Cl}$ & 2 & $\mathrm{~S}$ & 179 & 3780.350000 & 4.822217 & 1.000000 & 4.822217 \\
\hline $\mathrm{Cl}$ & 3 & $\mathrm{~S}$ & 179 & 860.474000 & 7.823932 & 1.000000 & 7.823932 \\
\hline $\mathrm{Cl}$ & 4 & $\mathrm{~S}$ & 179 & 242.145000 & 10.388253 & 1.000000 & 10.388253 \\
\hline $\mathrm{Cl}$ & 5 & $\mathrm{~S}$ & 179 & 77.334900 & 8.977789 & 1.000000 & 8.977789 \\
\hline $\mathrm{Cl}$ & 6 & $\mathrm{~S}$ & 179 & 26.247000 & 2.808761 & 1.000000 & 2.808761 \\
\hline $\mathrm{Cl}$ & 7 & $\mathrm{~S}$ & 180 & 491.765000 & -0.170987 & 1.000000 & -0.170987 \\
\hline $\mathrm{Cl}$ & 8 & $\mathrm{~S}$ & 180 & 116.984000 & -0.778641 & 1.000000 & -0.778641 \\
\hline $\mathrm{Cl}$ & 9 & $\mathrm{~S}$ & 180 & 37.415300 & -1.213271 & 1.000000 & -1.213271 \\
\hline $\mathrm{Cl}$ & 10 & $\mathrm{~S}$ & 180 & 13.783400 & 0.229508 & 1.000000 & 0.229508 \\
\hline $\mathrm{Cl}$ & 11 & $\mathrm{~S}$ & 180 & 5.452150 & 1.498690 & 1.000000 & 1.498690 \\
\hline $\mathrm{Cl}$ & 12 & $\mathrm{~S}$ & 180 & 2.225880 & 0.604202 & 1.000000 & 0.604202 \\
\hline \multirow[t]{3}{*}{$\mathrm{Cl}$} & 13 & $\mathrm{X}$ & 181 & 491.765000 & 13.168757 & 1.000000 & 13.168757 \\
\hline & & $\mathrm{Y}$ & 182 & & & 1.000000 & 13.168757 \\
\hline & & $\mathrm{z}$ & 183 & & & 1.000000 & 13.168757 \\
\hline \multirow[t]{3}{*}{$\mathrm{Cl}$} & 14 & $\mathrm{x}$ & 181 & 116.984000 & 16.626258 & 1.000000 & 16.626258 \\
\hline & & $\mathrm{Y}$ & 182 & & & 1.000000 & 16.626258 \\
\hline & & $\mathrm{z}$ & 183 & & & 1.000000 & 16.626258 \\
\hline \multirow[t]{3}{*}{$\mathrm{Cl}$} & 15 & $\mathrm{x}$ & 181 & 37.415300 & 17.131445 & 1.000000 & 17.131445 \\
\hline & & $\mathrm{Y}$ & 182 & & & 1.000000 & 17.131445 \\
\hline & & $\mathrm{z}$ & 183 & & & 1.000000 & 17.131445 \\
\hline \multirow[t]{3}{*}{$\mathrm{Cl}$} & 16 & $\mathrm{x}$ & 181 & 13.783400 & 12.414939 & 1.000000 & 12.414939 \\
\hline & & $\mathrm{Y}$ & 182 & & & 1.000000 & 12.414939 \\
\hline & & $\mathrm{z}$ & 183 & & & 1.000000 & 12.414939 \\
\hline \multirow[t]{3}{*}{$\mathrm{Cl}$} & 17 & $\mathrm{x}$ & 181 & 5.452150 & 5.385835 & 1.000000 & 5.385835 \\
\hline & & $\mathrm{Y}$ & 182 & & & 1.000000 & 5.385835 \\
\hline & & $\mathrm{z}$ & 183 & & & 1.000000 & 5.385835 \\
\hline \multirow[t]{3}{*}{$\mathrm{Cl}$} & 18 & $\mathrm{x}$ & 181 & 2.225880 & 0.977199 & 1.000000 & 0.977199 \\
\hline & & $\mathrm{Y}$ & 182 & & & 1.000000 & 0.977199 \\
\hline & & $\mathrm{z}$ & 183 & & & 1.000000 & 0.977199 \\
\hline $\mathrm{Cl}$ & 19 & $\mathrm{~S}$ & 184 & 3.186490 & -0.428054 & 1.000000 & -0.428054 \\
\hline $\mathrm{Cl}$ & 20 & $\mathrm{~S}$ & 184 & 1.144270 & 0.048564 & 1.000000 & 0.048564 \\
\hline $\mathrm{Cl}$ & 21 & $\mathrm{~S}$ & 184 & 0.420377 & 0.394476 & 1.000000 & 0.394476 \\
\hline $\mathrm{Cl}$ & 22 & $\mathrm{x}$ & 185 & 3.186490 & -0.086775 & 1.000000 & -0.086775 \\
\hline
\end{tabular}




\begin{tabular}{|c|c|c|c|c|c|c|c|}
\hline & & $Y$ & 186 & & & 1.000000 & -0.086775 \\
\hline & & $\mathrm{Z}$ & 187 & & & 1.000000 & -0.086775 \\
\hline $\mathrm{Cl}$ & 23 & $\mathrm{X}$ & 185 & 1.144270 & 0.545848 & 1.000000 & 0.545848 \\
\hline & & Y & 186 & & & 1.000000 & 0.545848 \\
\hline & & $\mathrm{Z}$ & 187 & & & 1.000000 & 0.545848 \\
\hline $\mathrm{Cl}$ & 24 & $\mathrm{X}$ & 185 & 0.420377 & 0.358735 & 1.000000 & 0.358735 \\
\hline & & $\mathrm{Y}$ & 186 & & & 1.000000 & 0.358735 \\
\hline & & $\mathrm{Z}$ & 187 & & & 1.000000 & 0.358735 \\
\hline $\mathrm{Cl}$ & 25 & S & 188 & 0.142657 & 0.165436 & 1.000000 & 0.165436 \\
\hline $\mathrm{Cl}$ & 26 & $\mathrm{X}$ & 189 & 0.142657 & 0.124970 & 1.000000 & 0.124970 \\
\hline & & Y & 190 & & & 1.000000 & 0.124970 \\
\hline & & $\mathrm{Z}$ & 191 & & & 1.000000 & 0.124970 \\
\hline $\mathrm{Cl}$ & 27 & $\mathrm{XX}$ & 192 & 0.750000 & 0.994871 & 1.000000 & 0.994871 \\
\hline & & YY & 193 & & & 1.000000 & 0.994871 \\
\hline & & $\mathrm{ZZ}$ & 194 & & & 1.000000 & 0.994871 \\
\hline & & $X Y$ & 195 & & & 1.732051 & 1.723167 \\
\hline & & $\mathrm{xz}$ & 196 & & & 1.732051 & 1.723167 \\
\hline & & $\mathrm{YZ}$ & 197 & & & 1.732051 & 1.723167 \\
\hline $\mathrm{H}$ & 1 & $S$ & 287 & 18.731137 & 0.214935 & 1.000000 & 0.214935 \\
\hline $\mathrm{H}$ & 2 & S & 287 & 2.825394 & 0.364571 & 1.000000 & 0.364571 \\
\hline $\mathrm{H}$ & 3 & $S$ & 287 & 0.640122 & 0.415051 & 1.000000 & 0.415051 \\
\hline $\mathrm{H}$ & 4 & S & 288 & 0.161278 & 0.181381 & 1.000000 & 0.181381 \\
\hline
\end{tabular}

\section{Basis Set of Single Point Energy Calculations (LACV3P**+)}

Gaussian Functions - Normalized coefficients

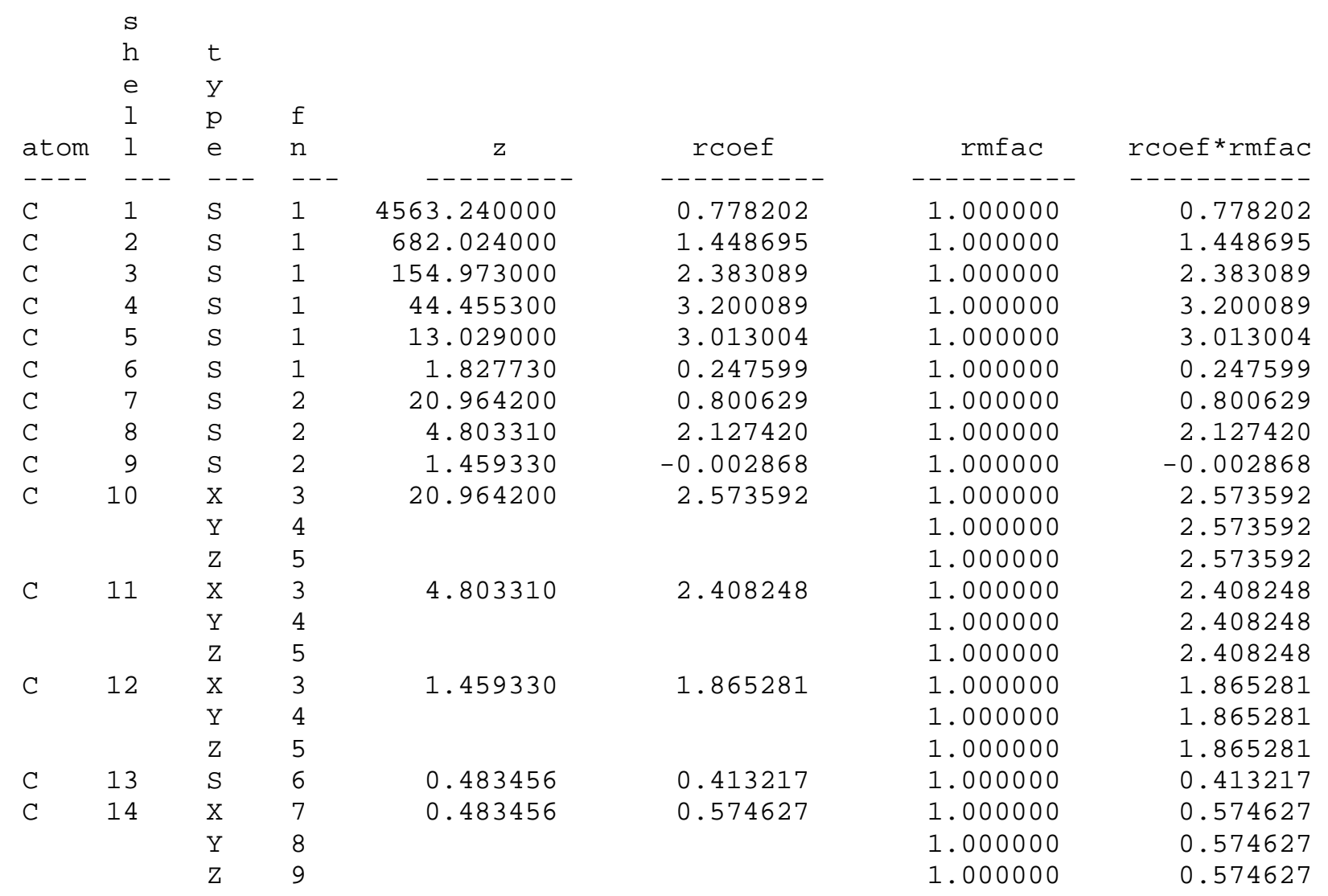




\begin{tabular}{|c|c|c|c|c|c|c|c|}
\hline $\mathrm{C}$ & 15 & S & 10 & 0.145585 & 0.167976 & 1.000000 & 0.167976 \\
\hline \multirow[t]{3}{*}{$\mathrm{C}$} & 16 & $\mathrm{X}$ & 11 & 0.145585 & 0.128185 & 1.000000 & 0.128185 \\
\hline & & Y & 12 & & & 1.000000 & 0.128185 \\
\hline & & $\mathrm{Z}$ & 13 & & & 1.000000 & 0.128185 \\
\hline \multirow[t]{6}{*}{ C } & 17 & $x X$ & 14 & 0.626000 & 0.725128 & 1.000000 & 0.725128 \\
\hline & & $Y Y$ & 15 & & & 1.000000 & 0.725128 \\
\hline & & $\mathrm{ZZ}$ & 16 & & & 1.000000 & 0.725128 \\
\hline & & $X Y$ & 17 & & & 1.732051 & 1.255958 \\
\hline & & $\mathrm{XZ}$ & 18 & & & 1.732051 & 1.255958 \\
\hline & & $Y Z$ & 19 & & & 1.732051 & 1.255958 \\
\hline $\mathrm{C}$ & 18 & S & 20 & 0.043800 & 0.068236 & 1.000000 & 0.068236 \\
\hline \multirow[t]{3}{*}{$\mathrm{C}$} & 19 & $\mathrm{X}$ & 21 & 0.043800 & 0.028562 & 1.000000 & 0.028562 \\
\hline & & Y & 22 & & & 1.000000 & 0.028562 \\
\hline & & Z & 23 & & & 1.000000 & 0.028562 \\
\hline $\mathrm{N}$ & 1 & $S$ & 24 & 6293.480000 & 0.991968 & 1.000000 & 0.991968 \\
\hline $\mathrm{N}$ & 2 & S & 24 & 949.044000 & 1.823240 & 1.000000 & 1.823240 \\
\hline $\mathrm{N}$ & 3 & $S$ & 24 & 218.776000 & 2.979891 & 1.000000 & 2.979891 \\
\hline $\mathrm{N}$ & 4 & $S$ & 24 & 63.691600 & 4.000007 & 1.000000 & 4.000007 \\
\hline $\mathrm{N}$ & 5 & $S$ & 24 & 18.828200 & 3.881008 & 1.000000 & 3.881008 \\
\hline $\mathrm{N}$ & 6 & $S$ & 24 & 2.720230 & 0.386764 & 1.000000 & 0.386764 \\
\hline $\mathrm{N}$ & 7 & $S$ & 25 & 30.633100 & 1.038501 & 1.000000 & 1.038501 \\
\hline $\mathrm{N}$ & 8 & $S$ & 25 & 7.026140 & 2.834794 & 1.000000 & 2.834794 \\
\hline $\mathrm{N}$ & 9 & S & 25 & 2.112050 & -0.003208 & 1.000000 & -0.003208 \\
\hline \multirow[t]{3}{*}{$\mathrm{N}$} & 10 & $\mathrm{X}$ & 26 & 30.633100 & 3.935616 & 1.000000 & 3.935616 \\
\hline & & Y & 27 & & & 1.000000 & 3.935616 \\
\hline & & $\mathrm{Z}$ & 28 & & & 1.000000 & 3.935616 \\
\hline \multirow[t]{3}{*}{$\mathrm{N}$} & 11 & $\mathrm{X}$ & 26 & 7.026140 & 3.870994 & 1.000000 & 3.870994 \\
\hline & & Y & 27 & & & 1.000000 & 3.870994 \\
\hline & & $\mathrm{Z}$ & 28 & & & 1.000000 & 3.870994 \\
\hline \multirow[t]{3}{*}{$\mathrm{N}$} & 12 & $\mathrm{X}$ & 26 & 2.112050 & 2.967271 & 1.000000 & 2.967271 \\
\hline & & $\mathrm{Y}$ & 27 & & & 1.000000 & 2.967271 \\
\hline & & $\mathrm{Z}$ & 28 & & & 1.000000 & 2.967271 \\
\hline $\mathrm{N}$ & 13 & S & 29 & 0.684009 & 0.536051 & 1.000000 & 0.536051 \\
\hline \multirow[t]{3}{*}{$\mathrm{N}$} & 14 & $\mathrm{X}$ & 30 & 0.684009 & 0.886681 & 1.000000 & 0.886681 \\
\hline & & $\mathrm{Y}$ & 31 & & & 1.000000 & 0.886681 \\
\hline & & $\mathrm{Z}$ & 32 & & & 1.000000 & 0.886681 \\
\hline $\mathrm{N}$ & 15 & $S$ & 33 & 0.200878 & 0.213850 & 1.000000 & 0.213850 \\
\hline \multirow[t]{3}{*}{$\mathrm{N}$} & 16 & $\mathrm{X}$ & 34 & 0.200878 & 0.191693 & 1.000000 & 0.191693 \\
\hline & & Y & 35 & & & 1.000000 & 0.191693 \\
\hline & & $\mathrm{Z}$ & 36 & & & 1.000000 & 0.191693 \\
\hline \multirow[t]{6}{*}{$\mathrm{N}$} & 17 & $\mathrm{XX}$ & 37 & 0.913000 & 1.403568 & 1.000000 & 1.403568 \\
\hline & & $Y Y$ & 38 & & & 1.000000 & 1.403568 \\
\hline & & $\mathrm{ZZ}$ & 39 & & & 1.000000 & 1.403568 \\
\hline & & $X Y$ & 40 & & & 1.732051 & 2.431050 \\
\hline & & $\mathrm{XZ}$ & 41 & & & 1.732051 & 2.431050 \\
\hline & & $Y Z$ & 42 & & & 1.732051 & 2.431050 \\
\hline $\mathrm{N}$ & 18 & S & 43 & 0.063900 & 0.090581 & 1.000000 & 0.090581 \\
\hline \multirow[t]{3}{*}{$\mathrm{N}$} & 19 & $\mathrm{X}$ & 44 & 0.063900 & 0.045795 & 1.000000 & 0.045795 \\
\hline & & Y & 45 & & & 1.000000 & 0.045795 \\
\hline & & Z & 46 & & & 1.000000 & 0.045795 \\
\hline $\mathrm{Ru}$ & 1 & $S$ & 116 & 2.565000 & -1.504161 & 1.000000 & -1.504161 \\
\hline $\mathrm{Ru}$ & 2 & $S$ & 116 & 1.508000 & 1.289616 & 1.000000 & 1.289616 \\
\hline $\mathrm{Ru}$ & 3 & S & 116 & 0.512900 & 0.242621 & 1.000000 & 0.242621 \\
\hline $\mathrm{Ru}$ & 4 & $S$ & 117 & 1.508000 & -0.284585 & 1.000000 & -0.284585 \\
\hline $\mathrm{Ru}$ & 5 & S & 117 & 0.512900 & -0.322652 & 1.000000 & -0.322652 \\
\hline $\mathrm{Ru}$ & 6 & $S$ & 118 & 0.136200 & 0.159788 & 1.000000 & 0.159788 \\
\hline Ru & 7 & S & 119 & 0.041700 & 0.065768 & 1.000000 & 0.065768 \\
\hline
\end{tabular}




\begin{tabular}{|c|c|c|c|c|c|c|c|}
\hline \multirow[t]{3}{*}{$\mathrm{Ru}$} & 8 & $\mathrm{X}$ & 120 & 4.859000 & -1.402272 & 1.000000 & -1.402272 \\
\hline & & $\mathrm{Y}$ & 121 & & & 1.000000 & -1.402272 \\
\hline & & $\mathrm{Z}$ & 122 & & & 1.000000 & -1.402272 \\
\hline \multirow[t]{3}{*}{$\mathrm{Ru}$} & 9 & $\mathrm{X}$ & 120 & 1.219000 & 1.957239 & 1.000000 & 1.957239 \\
\hline & & $\mathrm{Y}$ & 121 & & & 1.000000 & 1.957239 \\
\hline & & $\mathrm{Z}$ & 122 & & & 1.000000 & 1.957239 \\
\hline \multirow[t]{3}{*}{$\mathrm{Ru}$} & 10 & $\mathrm{X}$ & 123 & 0.441300 & 0.512693 & 1.000000 & 0.512693 \\
\hline & & $\mathrm{Y}$ & 124 & & & 1.000000 & 0.512693 \\
\hline & & $\mathrm{Z}$ & 125 & & & 1.000000 & 0.512693 \\
\hline \multirow[t]{3}{*}{$\mathrm{Ru}$} & 11 & $\mathrm{X}$ & 126 & 0.083000 & 0.063502 & 1.000000 & 0.063502 \\
\hline & & $\mathrm{Y}$ & 127 & & & 1.000000 & 0.063502 \\
\hline & & $\mathrm{Z}$ & 128 & & & 1.000000 & 0.063502 \\
\hline \multirow[t]{3}{*}{$\mathrm{Ru}$} & 12 & $\mathrm{X}$ & 129 & 0.025000 & 0.014170 & 1.000000 & 0.014170 \\
\hline & & $\mathrm{Y}$ & 130 & & & 1.000000 & 0.014170 \\
\hline & & $\mathrm{Z}$ & 131 & & & 1.000000 & 0.014170 \\
\hline \multirow[t]{6}{*}{$\mathrm{Ru}$} & 13 & $\mathrm{XX}$ & 132 & 4.105000 & 1.748105 & 1.000000 & 1.748105 \\
\hline & & $Y Y$ & 133 & & & 1.000000 & 1.748105 \\
\hline & & $\mathrm{ZZ}$ & 134 & & & 1.000000 & 1.748105 \\
\hline & & $X Y$ & 135 & & & 1.732051 & 3.027807 \\
\hline & & $\mathrm{XZ}$ & 136 & & & 1.732051 & 3.027807 \\
\hline & & $\mathrm{YZ}$ & 137 & & & 1.732051 & 3.027807 \\
\hline \multirow[t]{6}{*}{$\mathrm{Ru}$} & 14 & $\mathrm{XX}$ & 132 & 1.377000 & 2.716591 & 1.000000 & 2.716591 \\
\hline & & YY & 133 & & & 1.000000 & 2.716591 \\
\hline & & $\mathrm{ZZ}$ & 134 & & & 1.000000 & 2.716591 \\
\hline & & $X Y$ & 135 & & & 1.732051 & 4.705274 \\
\hline & & $\mathrm{XZ}$ & 136 & & & 1.732051 & 4.705274 \\
\hline & & $\mathrm{YZ}$ & 137 & & & 1.732051 & 4.705274 \\
\hline \multirow[t]{6}{*}{$\mathrm{Ru}$} & 15 & $\mathrm{XX}$ & 138 & 0.482800 & 0.460259 & 1.000000 & 0.460259 \\
\hline & & YY & 139 & & & 1.000000 & 0.460259 \\
\hline & & $\mathrm{ZZ}$ & 140 & & & 1.000000 & 0.460259 \\
\hline & & $X Y$ & 141 & & & 1.732051 & 0.797192 \\
\hline & & $\mathrm{XZ}$ & 142 & & & 1.732051 & 0.797192 \\
\hline & & $\mathrm{YZ}$ & 143 & & & 1.732051 & 0.797192 \\
\hline \multirow[t]{6}{*}{$\mathrm{Ru}$} & 16 & $\mathrm{XX}$ & 144 & 0.150100 & 0.059577 & 1.000000 & 0.059577 \\
\hline & & YY & 145 & & & 1.000000 & 0.059577 \\
\hline & & $\mathrm{ZZ}$ & 146 & & & 1.000000 & 0.059577 \\
\hline & & $X Y$ & 147 & & & 1.732051 & 0.103190 \\
\hline & & $\mathrm{XZ}$ & 148 & & & 1.732051 & 0.103190 \\
\hline & & $\mathrm{YZ}$ & 149 & & & 1.732051 & 0.103190 \\
\hline \multirow[t]{6}{*}{$\mathrm{Ru}$} & 17 & $\mathrm{XX}$ & 150 & 0.042000 & 0.006414 & 1.000000 & 0.006414 \\
\hline & & YY & 151 & & & 1.000000 & 0.006414 \\
\hline & & $\mathrm{ZZ}$ & 152 & & & 1.000000 & 0.006414 \\
\hline & & $\mathrm{XY}$ & 153 & & & 1.732051 & 0.011109 \\
\hline & & $\mathrm{XZ}$ & 154 & & & 1.732051 & 0.011109 \\
\hline & & $\mathrm{YZ}$ & 155 & & & 1.732051 & 0.011109 \\
\hline $\mathrm{P}$ & 1 & $S$ & 156 & 77492.400000 & 2.585268 & 1.000000 & 2.585268 \\
\hline $\mathrm{P}$ & 2 & $S$ & 156 & 11605.800000 & 4.835730 & 1.000000 & 4.835730 \\
\hline $\mathrm{P}$ & 3 & $S$ & 156 & 2645.960000 & 8.193040 & 1.000000 & 8.193040 \\
\hline $\mathrm{P}$ & 4 & $S$ & 156 & 754.976000 & 12.670224 & 1.000000 & 12.670224 \\
\hline $\mathrm{P}$ & 5 & $S$ & 156 & 248.755000 & 16.883827 & 1.000000 & 16.883827 \\
\hline $\mathrm{P}$ & 6 & $S$ & 156 & 91.156500 & 11.842990 & 1.000000 & 11.842990 \\
\hline $\mathrm{P}$ & 7 & $S$ & 157 & 91.156500 & 3.369477 & 1.000000 & 3.369477 \\
\hline $\mathrm{P}$ & 8 & $S$ & 157 & 36.225700 & 6.605233 & 1.000000 & 6.605233 \\
\hline $\mathrm{P}$ & 9 & $S$ & 157 & 15.211300 & 1.448406 & 1.000000 & 1.448406 \\
\hline $\mathrm{P}$ & 10 & $S$ & 158 & 4.794170 & 2.309113 & 1.000000 & 2.309113 \\
\hline $\mathrm{P}$ & 11 & S & 159 & 1.807930 & 1.111210 & 1.000000 & 1.111210 \\
\hline $\mathrm{P}$ & 12 & $S$ & 160 & 0.356816 & 0.329036 & 1.000000 & 0.329036 \\
\hline $\mathrm{P}$ & 13 & $S$ & 161 & 0.114783 & 0.140546 & 1.000000 & 0.140546 \\
\hline
\end{tabular}




\begin{tabular}{|c|c|c|c|c|c|c|c|}
\hline \multirow[t]{3}{*}{$\mathrm{P}$} & 14 & $\mathrm{X}$ & 162 & 384.843000 & 22.367400 & 1.000000 & 22.367400 \\
\hline & & $Y$ & 163 & & & 1.000000 & 22.367400 \\
\hline & & $\mathrm{Z}$ & 164 & & & 1.000000 & 22.367400 \\
\hline \multirow[t]{3}{*}{$\mathrm{P}$} & 15 & $\mathrm{X}$ & 162 & 90.552100 & 27.821388 & 1.000000 & 27.821388 \\
\hline & & $\mathrm{Y}$ & 163 & & & 1.000000 & 27.821388 \\
\hline & & $\mathrm{Z}$ & 164 & & & 1.000000 & 27.821388 \\
\hline \multirow[t]{3}{*}{$\mathrm{P}$} & 16 & $\mathrm{X}$ & 162 & 29.133900 & 28.217572 & 1.000000 & 28.217572 \\
\hline & & $Y$ & 163 & & & 1.000000 & 28.217572 \\
\hline & & $\mathrm{Z}$ & 164 & & & 1.000000 & 28.217572 \\
\hline \multirow[t]{3}{*}{$\mathrm{P}$} & 17 & $\mathrm{X}$ & 162 & 10.886200 & 20.522404 & 1.000000 & 20.522404 \\
\hline & & $\mathrm{Y}$ & 163 & & & 1.000000 & 20.522404 \\
\hline & & $\mathrm{Z}$ & 164 & & & 1.000000 & 20.522404 \\
\hline \multirow[t]{3}{*}{$\mathrm{P}$} & 18 & $\mathrm{X}$ & 165 & 4.352590 & 5.630872 & 1.000000 & 5.630872 \\
\hline & & $\mathrm{Y}$ & 166 & & & 1.000000 & 5.630872 \\
\hline & & $\mathrm{Z}$ & 167 & & & 1.000000 & 5.630872 \\
\hline \multirow[t]{3}{*}{$\mathrm{P}$} & 19 & $\mathrm{X}$ & 165 & 1.777060 & 1.251861 & 1.000000 & 1.251861 \\
\hline & & $\mathrm{Y}$ & 166 & & & 1.000000 & 1.251861 \\
\hline & & $\mathrm{Z}$ & 167 & & & 1.000000 & 1.251861 \\
\hline \multirow[t]{3}{*}{$\mathrm{P}$} & 20 & $\mathrm{X}$ & 168 & 0.697005 & 0.907789 & 1.000000 & 0.907789 \\
\hline & & $Y$ & 169 & & & 1.000000 & 0.907789 \\
\hline & & $\mathrm{Z}$ & 170 & & & 1.000000 & 0.907789 \\
\hline \multirow[t]{3}{*}{$\mathrm{P}$} & 21 & $\mathrm{X}$ & 171 & 0.253532 & 0.256437 & 1.000000 & 0.256437 \\
\hline & & $\mathrm{Y}$ & 172 & & & 1.000000 & 0.256437 \\
\hline & & $\mathrm{Z}$ & 173 & & & 1.000000 & 0.256437 \\
\hline \multirow[t]{3}{*}{$\mathrm{P}$} & 22 & $\mathrm{X}$ & 174 & 0.068493 & 0.049946 & 1.000000 & 0.049946 \\
\hline & & $\mathrm{Y}$ & 175 & & & 1.000000 & 0.049946 \\
\hline & & $\mathrm{Z}$ & 176 & & & 1.000000 & 0.049946 \\
\hline \multirow[t]{6}{*}{$\mathrm{P}$} & 23 & $\mathrm{XX}$ & 177 & 0.550000 & 0.578155 & 1.000000 & 0.578155 \\
\hline & & $Y Y$ & 178 & & & 1.000000 & 0.578155 \\
\hline & & $\mathrm{ZZ}$ & 179 & & & 1.000000 & 0.578155 \\
\hline & & $X Y$ & 180 & & & 1.732051 & 1.001394 \\
\hline & & $\mathrm{XZ}$ & 181 & & & 1.732051 & 1.001394 \\
\hline & & $\mathrm{YZ}$ & 182 & & & 1.732051 & 1.001394 \\
\hline $\mathrm{P}$ & 24 & $S$ & 183 & 0.034800 & 0.057424 & 1.000000 & 0.057424 \\
\hline \multirow[t]{3}{*}{$\mathrm{P}$} & 25 & $\mathrm{X}$ & 184 & 0.034800 & 0.021425 & 1.000000 & 0.021425 \\
\hline & & $\mathrm{Y}$ & 185 & & & 1.000000 & 0.021425 \\
\hline & & $\mathrm{Z}$ & 186 & & & 1.000000 & 0.021425 \\
\hline $\mathrm{Cl}$ & 1 & S & 279 & 105819.000000 & 3.085953 & 1.000000 & 3.085953 \\
\hline $\mathrm{Cl}$ & 2 & S & 279 & 15872.000000 & 5.762728 & 1.000000 & 5.762728 \\
\hline $\mathrm{Cl}$ & 3 & $S$ & 279 & 3619.650000 & 9.809764 & 1.000000 & 9.809764 \\
\hline $\mathrm{Cl}$ & 4 & S & 279 & 1030.800000 & 15.206778 & 1.000000 & 15.206778 \\
\hline $\mathrm{Cl}$ & 5 & S & 279 & 339.908000 & 20.477497 & 1.000000 & 20.477497 \\
\hline $\mathrm{Cl}$ & 6 & S & 279 & 124.538000 & 15.520664 & 1.000000 & 15.520664 \\
\hline $\mathrm{Cl}$ & 7 & S & 280 & 124.538000 & 3.565041 & 1.000000 & 3.565041 \\
\hline $\mathrm{Cl}$ & 8 & S & 280 & 49.513500 & 8.304458 & 1.000000 & 8.304458 \\
\hline $\mathrm{Cl}$ & 9 & S & 280 & 20.805600 & 2.025653 & 1.000000 & 2.025653 \\
\hline $\mathrm{Cl}$ & 10 & S & 281 & 6.583460 & 2.929212 & 1.000000 & 2.929212 \\
\hline $\mathrm{Cl}$ & 11 & S & 282 & 2.564680 & 1.444392 & 1.000000 & 1.444392 \\
\hline $\mathrm{Cl}$ & 12 & $S$ & 283 & 0.559763 & 0.461225 & 1.000000 & 0.461225 \\
\hline $\mathrm{Cl}$ & 13 & S & 284 & 0.183273 & 0.199634 & 1.000000 & 0.199634 \\
\hline \multirow[t]{3}{*}{$\mathrm{Cl}$} & 14 & $\mathrm{X}$ & 285 & 589.776000 & 9.905547 & 1.000000 & 9.905547 \\
\hline & & $\mathrm{Y}$ & 286 & & & 1.000000 & 9.905547 \\
\hline & & $\mathrm{Z}$ & 287 & & & 1.000000 & 9.905547 \\
\hline \multirow[t]{3}{*}{$\mathrm{Cl}$} & 15 & $\mathrm{X}$ & 285 & 139.849000 & 12.684690 & 1.000000 & 12.684690 \\
\hline & & $\mathrm{Y}$ & 286 & & & 1.000000 & 12.684690 \\
\hline & & $\mathrm{Z}$ & 287 & & & 1.000000 & 12.684690 \\
\hline \multirow[t]{2}{*}{$\mathrm{Cl}$} & 16 & $\mathrm{X}$ & 285 & 45.141300 & 13.572482 & 1.000000 & 13.572482 \\
\hline & & $\mathrm{Y}$ & 286 & & & 1.000000 & 13.572482 \\
\hline
\end{tabular}




\begin{tabular}{|c|c|c|c|c|c|c|c|}
\hline & & $\mathrm{Z}$ & 287 & & & 1.000000 & 13.572482 \\
\hline \multirow[t]{3}{*}{$\mathrm{Cl}$} & 17 & $\mathrm{X}$ & 285 & 16.873300 & 10.799801 & 1.000000 & 10.799801 \\
\hline & & $\mathrm{Y}$ & 286 & & & 1.000000 & 10.799801 \\
\hline & & $\mathrm{Z}$ & 287 & & & 1.000000 & 10.799801 \\
\hline \multirow[t]{3}{*}{$\mathrm{Cl}$} & 18 & $\mathrm{X}$ & 285 & 6.741100 & 11.961641 & 1.000000 & 11.961641 \\
\hline & & $\mathrm{Y}$ & 286 & & & 1.000000 & 11.961641 \\
\hline & & $\mathrm{Z}$ & 287 & & & 1.000000 & 11.961641 \\
\hline \multirow[t]{3}{*}{$\mathrm{Cl}$} & 19 & $\mathrm{X}$ & 288 & 6.741100 & -24.342963 & 1.000000 & -24.342963 \\
\hline & & $\mathrm{Y}$ & 289 & & & 1.000000 & -24.342963 \\
\hline & & $\mathrm{Z}$ & 290 & & & 1.000000 & -24.342963 \\
\hline \multirow[t]{3}{*}{$\mathrm{Cl}$} & 20 & $\mathrm{X}$ & 288 & 2.771520 & 5.058474 & 1.000000 & 5.058474 \\
\hline & & $\mathrm{Y}$ & 289 & & & 1.000000 & 5.058474 \\
\hline & & $\mathrm{Z}$ & 290 & & & 1.000000 & 5.058474 \\
\hline \multirow[t]{3}{*}{$\mathrm{Cl}$} & 21 & $\mathrm{X}$ & 291 & 1.023870 & 1.468068 & 1.000000 & 1.468068 \\
\hline & & $\mathrm{Y}$ & 292 & & & 1.000000 & 1.468068 \\
\hline & & $\mathrm{Z}$ & 293 & & & 1.000000 & 1.468068 \\
\hline \multirow[t]{3}{*}{$\mathrm{Cl}$} & 22 & $\mathrm{X}$ & 294 & 0.381368 & 0.427189 & 1.000000 & 0.427189 \\
\hline & & $\mathrm{Y}$ & 295 & & & 1.000000 & 0.427189 \\
\hline & & $\mathrm{Z}$ & 296 & & & 1.000000 & 0.427189 \\
\hline \multirow[t]{3}{*}{$\mathrm{Cl}$} & 23 & $\mathrm{X}$ & 297 & 0.109437 & 0.089721 & 1.000000 & 0.089721 \\
\hline & & $\mathrm{Y}$ & 298 & & & 1.000000 & 0.089721 \\
\hline & & $\mathrm{Z}$ & 299 & & & 1.000000 & 0.089721 \\
\hline \multirow[t]{6}{*}{$\mathrm{Cl}$} & 24 & $\mathrm{XX}$ & 300 & 0.750000 & 0.994871 & 1.000000 & 0.994871 \\
\hline & & $Y Y$ & 301 & & & 1.000000 & 0.994871 \\
\hline & & $\mathrm{ZZ}$ & 302 & & & 1.000000 & 0.994871 \\
\hline & & $X Y$ & 303 & & & 1.732051 & 1.723167 \\
\hline & & $\mathrm{XZ}$ & 304 & & & 1.732051 & 1.723167 \\
\hline & & $\mathrm{YZ}$ & 305 & & & 1.732051 & 1.723167 \\
\hline $\mathrm{Cl}$ & 25 & $S$ & 306 & 0.048300 & 0.073429 & 1.000000 & 0.073429 \\
\hline \multirow[t]{3}{*}{$\mathrm{Cl}$} & 26 & $\mathrm{X}$ & 307 & 0.048300 & 0.032276 & 1.000000 & 0.032276 \\
\hline & & $\mathrm{Y}$ & 308 & & & 1.000000 & 0.032276 \\
\hline & & $\mathrm{Z}$ & 309 & & & 1.000000 & 0.032276 \\
\hline $\mathrm{H}$ & 1 & $S$ & 507 & 33.865000 & 0.255069 & 1.000000 & 0.255069 \\
\hline $\mathrm{H}$ & 2 & $S$ & 507 & 5.094790 & 0.460109 & 1.000000 & 0.460109 \\
\hline $\mathrm{H}$ & 3 & S & 507 & 1.158790 & 0.678321 & 1.000000 & 0.678321 \\
\hline $\mathrm{H}$ & 4 & $S$ & 508 & 0.325840 & 0.307371 & 1.000000 & 0.307371 \\
\hline $\mathrm{H}$ & 5 & S & 509 & 0.102741 & 0.129336 & 1.000000 & 0.129336 \\
\hline \multirow[t]{3}{*}{$\mathrm{H}$} & 6 & $\mathrm{X}$ & 510 & 0.750000 & 0.994871 & 1.000000 & 0.994871 \\
\hline & & $\mathrm{Y}$ & 511 & & & 1.000000 & 0.994871 \\
\hline & & $\mathrm{Z}$ & 512 & & & 1.000000 & 0.994871 \\
\hline
\end{tabular}

